\title{
Access to Chiral Chromenones through Organocatalyzed Mannich/Annulation Sequence
}

Jingxiang Duan, ${ }^{\mathrm{a}}$ Zongli Xiong, ${ }^{\mathrm{a}}$ Yuqiao Zhou, ${ }^{\mathrm{b}}$ Weijun Yao, ${ }^{\mathrm{c}}$ Xiaoyi Li, ${ }^{\mathrm{a}}$ Min Zhang, ${ }^{a}$ Zhen Wang*a

${ }^{\text {a }}$ School of Pharmaceutical Sciences and Chongqing Key Laboratory of Natural Drug Research, Chongqing University, Chongqing 401331, P. R. China.

E-mail: wangz1114@cqu.edu.cn (Zhen Wang)

${ }^{\mathrm{b}}$ Key Laboratory of Green Chemistry \& Technology, Ministry of Education, College of Chemistry, Sichuan University, Chengdu 610064, P. R. China.

${ }^{\mathrm{c}}$ Department of Chemistry, Zhejiang Sci-Tech University, Hangzhou, 310018, P. R. China. 


\section{Table of contents}

1. General Information.................................................... 1

2. Control experiments and proposed mechanisms.............................

3. Typical experimental procedure for asymmetric synthesis of chromone derivatives.......................................................... 4

4. The procedure for scaled-up reaction.................................... 4

5. The procedure for further transformation of products.........................5

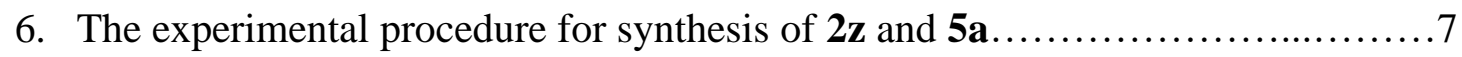

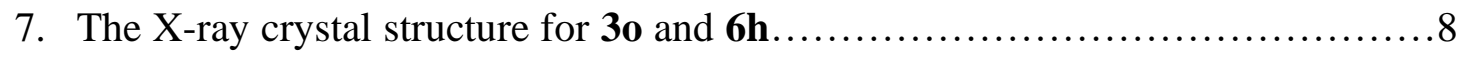

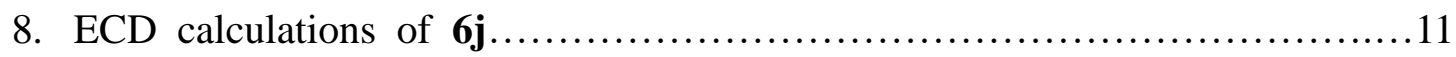

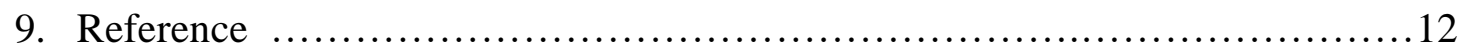

10. The analytical and spectral characterization data for the products................13

11. Copies of NMR spectra............................................... 72 


\section{General Information:}

${ }^{1} \mathrm{H}$ NMR spectra were recorded at 400 or $500 \mathrm{~Hz}$. The chemical shifts were recorded in ppm relative to tetramethylsilane and with the solvent resonance as the internal standard. Data were reported as follows: chemical shift, multiplicity ( $\mathrm{s}=$ singlet, $\mathrm{d}=$ doublet, $\mathrm{t}=$ triplet, $\mathrm{m}=$ multiplet, $\mathrm{br}=$ broad $)$, coupling constants $(\mathrm{Hz})$, integration . ${ }^{13} \mathrm{C}$ NMR data were collected at 100 or $150 \mathrm{MHz}$ with complete proton decoupling.

Enantiomeric excesses (ee's) were determined by chiral HPLC analysis on Daicel Chiralcel IA, IB, IC, and ID columns in comparison with the authentic racemates. Optical rotations were reported as follows: $[\alpha]_{\mathrm{D}}^{\mathrm{T}}$ (c: $\mathrm{g} / 100 \mathrm{~mL}$, in solvent $\mathrm{CH}_{2} \mathrm{Cl}_{2}$ ). The high-resolution mass spectra (HRMS) were recorded on a commercial apparatus (FTMS+c ESI) or a Waters XEVO G2-S TOF Premier using ESI ionization. 1-(2-Hydroxyaryl)-1,3-diketones $\mathbf{1}$ derivatives were prepared according to the literature. ${ }^{1-3}$ Propargylamine 2 derivatives were prepared according to the literature. ${ }^{4,5}$

\section{Control experiments and proposed mechanisms}

\section{Control experiments}

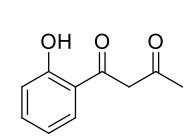

$1 \mathrm{a}, 0.2 \mathrm{mmol}$

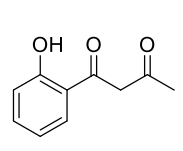

1a

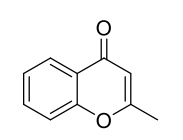

$3 a^{\prime}$

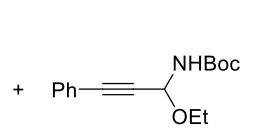

2a, $0.3 \mathrm{mmol}$

C4 (10 mol\%),

$\mathrm{Na}_{2} \mathrm{SO}_{4}(30 \mathrm{mg})$, toluene, $70^{\circ} \mathrm{C}, 48 \mathrm{~h}$ $95 \%$ yield

$\mathrm{C4}(10 \mathrm{~mol} \%)$,
$\mathrm{Na}_{2} \mathrm{SO}_{4}(30 \mathrm{mg})$, OEt

2a

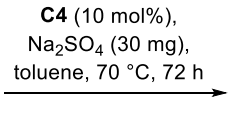

$\mathrm{Na}_{2} \mathrm{SO}_{4}(30 \mathrm{mg})$,
toluene, $70{ }^{\circ} \mathrm{C}, 48 \mathrm{~h}$

No Reaction

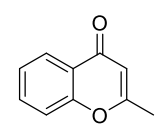

$3 a^{\prime}$

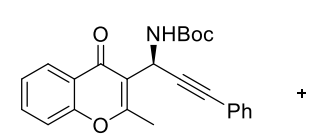

3a, $90 \%$ yield

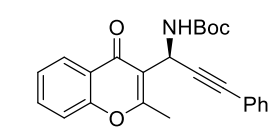

(a)

(b)

(c)

Under the standard reaction conditions, the cyclization product 3a' could be obtained in good yield. However, compound 3a' failed to transform to product 3a under the same conditions. 
Proposed mechanisms and HRMS analysis of the catalytic reaction

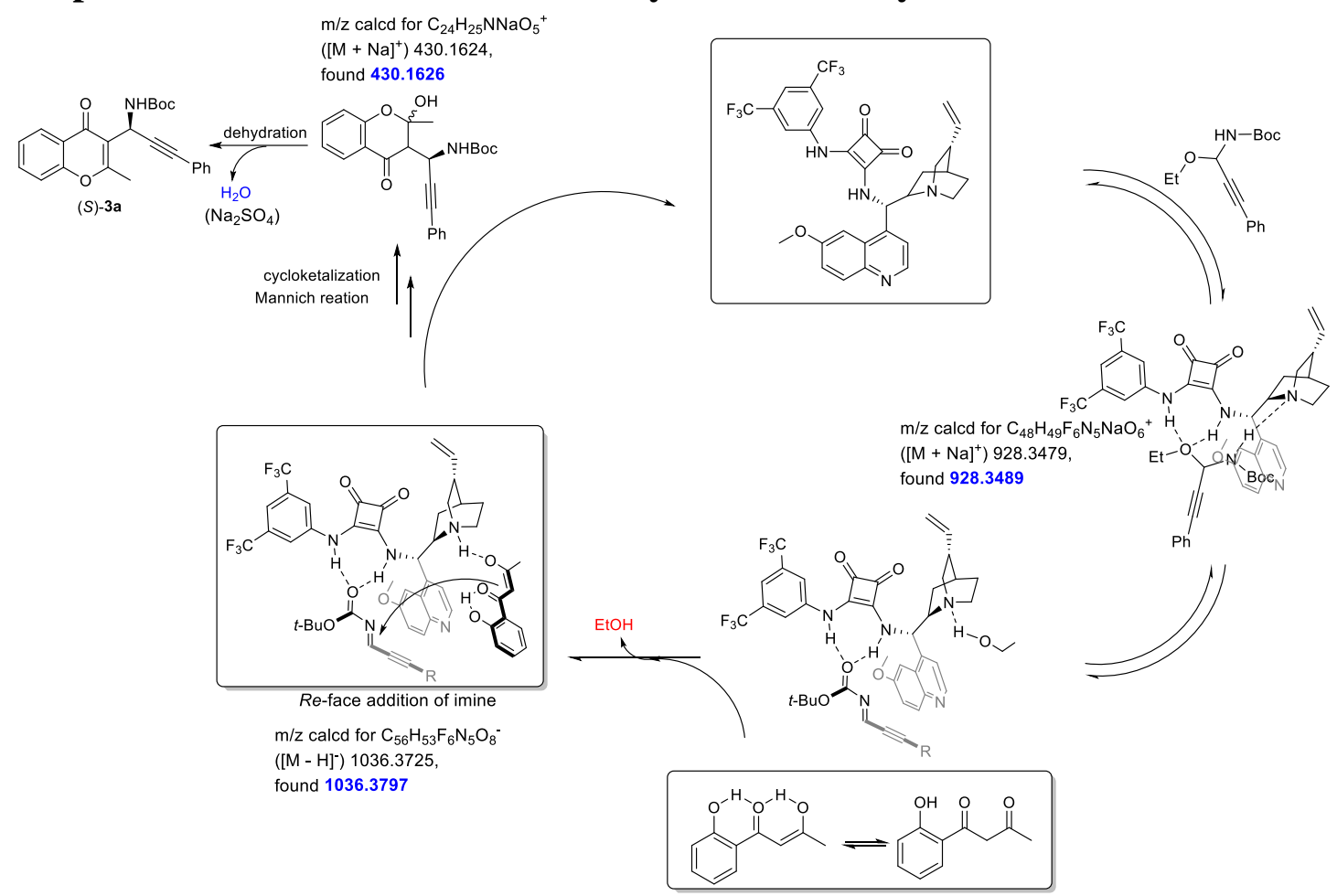

HRMS analysis of the reaction mixture

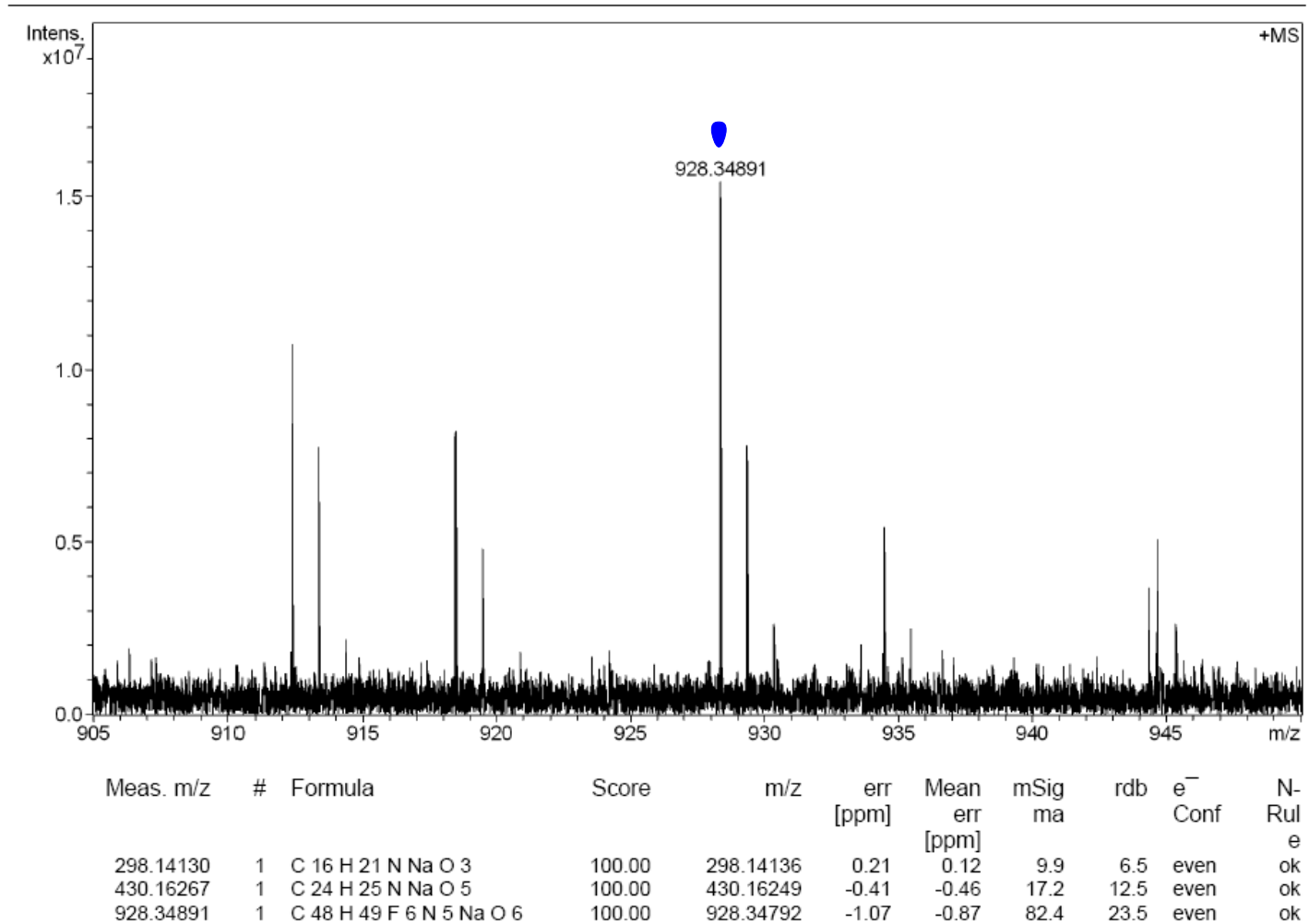



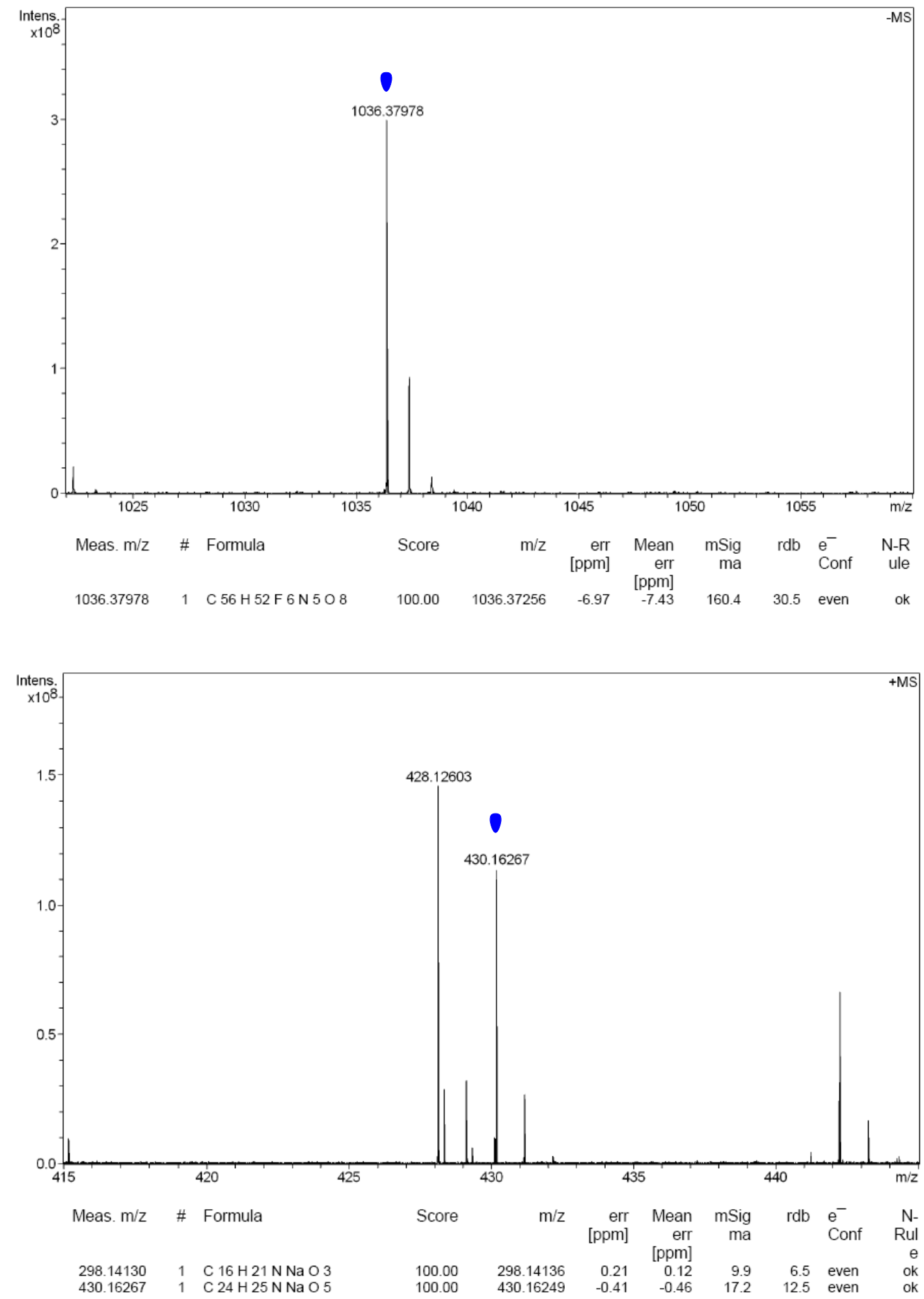


\section{Typical experimental procedure for asymmetric synthesis of chromone derivatives}
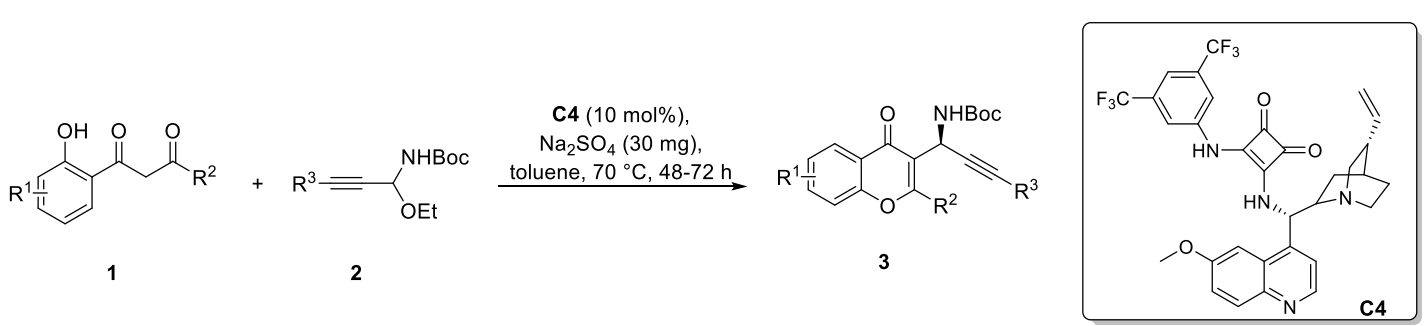

To a dried test tube containing a solution of 1-(2-hydroxyaryl)-1,3-diketones 1 (0.2 mmol, 1 equiv.) and $\mathbf{C 4}(0.02 \mathrm{mmol}, 10 \mathrm{~mol} \%)$ dissolved in toluene $(2.0 \mathrm{~mL}, 0.1 \mathrm{M})$ was added $\mathrm{Na}_{2} \mathrm{SO}_{4}(30 \mathrm{mg})$ and propargylamine $2(0.3 \mathrm{mmol}, 1.5$ equiv. $)$ at room temperature. The reaction was stirred at $70{ }^{\circ} \mathrm{C}$ (aluminum heating block for tubes) for 48-72 hours, and monitored by TLC. The mixture was then purified by column chromatography on silica gel with petroleum ether/ethyl acetate $(15: 1$ to $10: 1$, v/v) to obtain the products 3 determined by ${ }^{1} \mathrm{H}$ NMR. The ee values were determined by HPLC analysis on a chiral stationary phase.

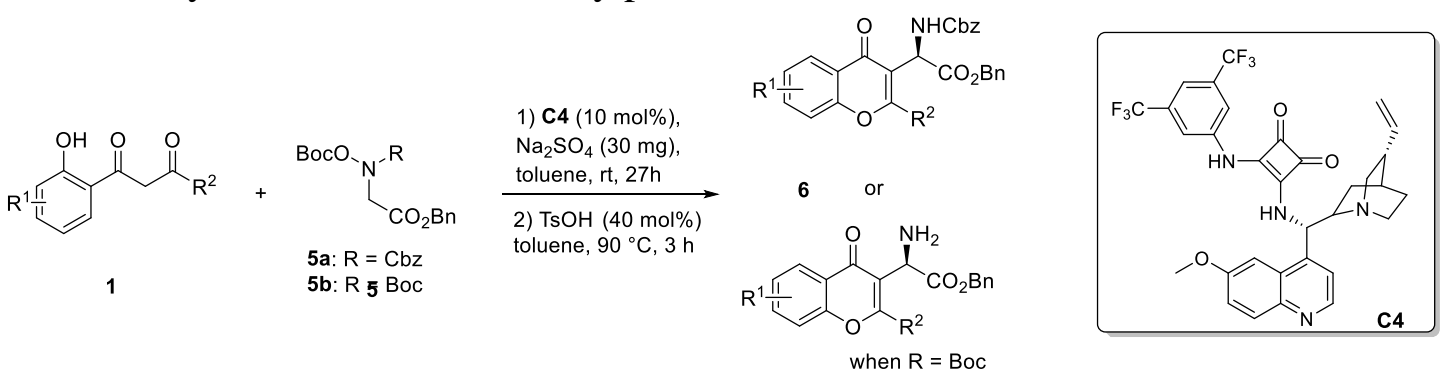

To a dried test tube containing a solution of 1-(2-hydroxyaryl)-1,3-diketones $\mathbf{1}(0.2$ mmol, 1 equiv.) and $\mathbf{C 4}(0.02 \mathrm{mmol}, 10 \mathrm{~mol} \%)$ dissolved in toluene $(2.0 \mathrm{~mL}, 0.1 \mathrm{M})$ was added $\mathrm{Na}_{2} \mathrm{SO}_{4}(30 \mathrm{mg})$ and $\mathrm{N}$-Cbz-O-Boc hydroxylamine 5a $(0.24 \mathrm{mmol}, 1.2$ equiv.) at room temperature. The reaction was stirred at room temperature for 27 hours, and monitored by TLC. Then, the mixture was initially purified by column chromatography on silica gel with petroleum ether/ethyl acetate $(10: 1, \mathrm{v} / \mathrm{v})$ to obtain the intermediate (When $\mathbf{5 b}$ was used, no initially purified was performed). $\mathrm{TsOH}$ (40 mol\%) was added to the solution of crude intermediate in toluene $(1.0 \mathrm{~mL})$, then, the mixture was heated at $90{ }^{\circ} \mathrm{C}$ (aluminum heating block for tubes) for 3 hours and monitored by TLC. Finally, the mixture was purified by column chromatography on silica gel with petroleum ether/ethyl acetate $(3: 1, \mathrm{v} / \mathrm{v})$ to obtain product 6 determined by ${ }^{1} \mathrm{H}$ NMR. The ee values were determined by HPLC analysis on a chiral stationary phase.

\section{The procedure for scaled-up reaction}

\section{Synthesis of 3a}

To a dried round bottom flask containing tert-butyl (1-ethoxy-3-phenylprop-2-yn-1-yl)carbamate $2 \mathbf{a}$ (550.7 $\mathrm{mg}, 2 \mathrm{mmol}$ ) and C4 (10 mol\%, $126.1 \mathrm{mg}$ ) dissolved in $10.0 \mathrm{~mL}$ toluene was added $\mathrm{Na}_{2} \mathrm{SO}_{4}(300.0 \mathrm{mg})$ and 
1-(2-hydroxyphenyl)butane-1,3-dione $\mathbf{1 a}(3 \mathrm{mmol}, 534.6 \mathrm{mg})$ at room temperature. The reaction mixture was stirred at $70{ }^{\circ} \mathrm{C}$ (oil bath) for 66 hours, and the mixture was purified by column chromatography on silica gel with petroleum ether/ethyl acetate $(15: 1$ to $10: 1, \mathrm{v} / \mathrm{v})$ to obtain the product $\mathbf{3 a}(662.1 \mathrm{mg}, 85 \%$ yield, $95 \%$ ee).

\section{Synthesis of 3y}

To a dried round bottom flask containing tert-butyl (1-ethoxy-3-(trimethylsilyl)prop-2-yn-1-yl)carbamate $\mathbf{2 y}(542.9 \mathrm{mg}, 2 \mathrm{mmol}$ ) and C4 (5 mol\%, $63.1 \mathrm{mg}$ ) dissolved in $10.0 \mathrm{~mL}$ toluene was added $\mathrm{Na}_{2} \mathrm{SO}_{4}(300.0 \mathrm{mg}$ ) and 1-(2-hydroxyphenyl)butane-1,3-dione $1 \mathrm{a}(3 \mathrm{mmol}, 534.6 \mathrm{mg})$ at room temperature. The reaction mixture was stirred at $70{ }^{\circ} \mathrm{C}$ (oil bath) for 72 hours, and the mixture was purified by column chromatography on silica gel with petroleum ether/ethyl acetate (15:1 to 10:1, v/v) to abtain the product $\mathbf{3 y}(501.4 \mathrm{mg}, 65 \%$ yield, 95\% ee).

\section{The procedure for further transformation of products}

\section{Synthesis of $\mathbf{3 z}$}

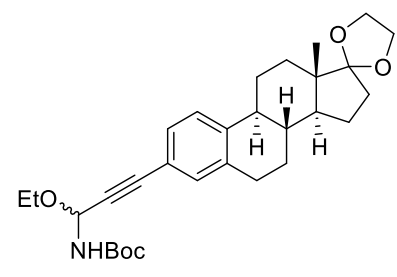

$2 z$

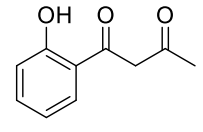

$1 \mathrm{a}$

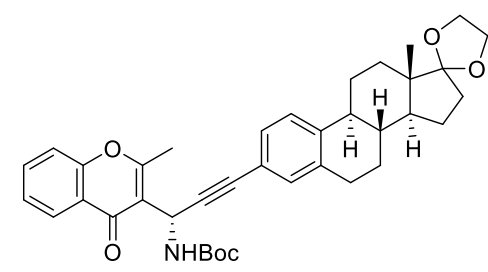

3z: $86 \%$ yield, $>19: 1 \mathrm{dr}$

To a dried test tube containing a solution of propargylamine $\mathbf{2 z}$ ( $0.05 \mathrm{mmol}, 1$ equiv.), $\mathrm{Na}_{2} \mathrm{SO}_{4}(10.0 \mathrm{mg})$ and $\mathbf{C 4}(10 \mathrm{~mol} \%)$ dissolved in toluene $(1.0 \mathrm{~mL})$ was added 1-(2-hydroxyaryl)-1,3-diketones $\mathbf{1 a}(0.075 \mathrm{mmol}, 1.5$ equiv.) at room temperature. The reaction was stirred at $80{ }^{\circ} \mathrm{C}$ (aluminum heating block for tubes) for 62 hours, and monitored by TLC. The mixture was purified by column chromatography on silica gel with petroleum ether/ethyl acetate (15:1 to $10: 1$, v/v) to obtain the products $\mathbf{3 z}$ (26.3 $\mathrm{mg}, 86 \%$ yield, $>19: 1 \mathrm{dr}$ ) as yellow oil.

\section{Synthesis of $\mathbf{6 j}$}

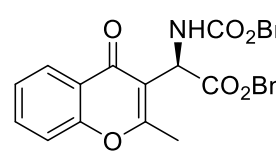

$6 a$

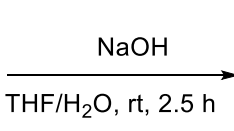

$\mathrm{THF} / \mathrm{H}_{2} \mathrm{O}, \mathrm{rt}, 2.5 \mathrm{~h}$<smiles>Cc1oc2ccccc2c(=O)c1[C@H](NC(=O)OCc1ccccc1)C(=O)O</smiles>

6j: $84 \%$ yield

To a solution of $\mathbf{6 a}(120 \mathrm{mg}, 0.26 \mathrm{mmol}, 1.0$ equiv.) in $5.0 \mathrm{~mL}$ THF was added $\mathrm{NaOH}$ (5 equiv. in $2.0 \mathrm{~mL}$ water). The reaction was stirred at $26{ }^{\circ} \mathrm{C}$ for 2.5 hours, and monitored by TLC. Then, the mixture was adjusted $\mathrm{pH}$ to $2-4$ with $1 \mathrm{M} \mathrm{HCl}$ and extracted with EA. The combined organic layers were washed with saturated brine, dried over $\mathrm{Na}_{2} \mathrm{SO}_{4}$, and the solvent was evaporated under reduced pressure, purified by column chromatography on silica gel with dichloromethane/methanol (10:1) to 
obtain the product $\mathbf{6 j}$ ( $80.0 \mathrm{mg}, 84 \%$ yield) as colorless oil.

\section{Synthesis of $7 \mathrm{a}$}
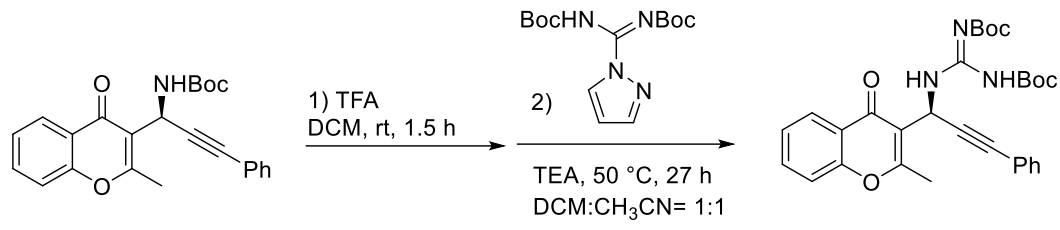

$3 a$

$7 a: 65 \%$ yield, $94 \%$ ee

To a solution of 3a (77.9 mg, 1.0 equiv.) in $2 \mathrm{~mL}$ DCM was added trifluoroacetic acid $(0.5 \mathrm{~mL})$, the reaction was stirred at room temperature for 1.5 hours, and monitored by TLC. The solvent was evaporated under reduced pressure and the crude mixture was redissolved in $2.0 \mathrm{~mL} \mathrm{DCM} / \mathrm{CH}_{3} \mathrm{CN}(1: 1, \mathrm{v} / \mathrm{v})$, followed by the addition of $N, N$-di-boc-1 $H$-pyrazole-1-carboxamidine $(74.5 \mathrm{mg})$ and triethylamine $(140 \mu \mathrm{L})$. The mixture was stirred at $50{ }^{\circ} \mathrm{C}$ (aluminum heating block for tubes) for 27 hours, and monitored by TLC. The solution was evaporated under reduced pressure and the residue was purified by column chromatography on silica gel with petroleum ether/ethyl acetate $(10: 1)$ to obtain the product $7 \mathbf{a}(72.3 \mathrm{mg}, 68 \%$ yield, $94 \%$ ee) as yellow oil.

\section{Synthesis of 8a}

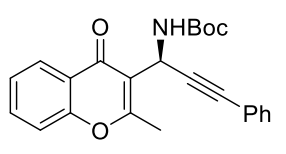

3a

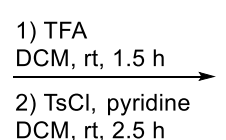

DCM, rt, $2.5 \mathrm{~h}$

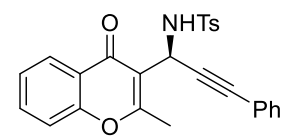

8 a: $65 \%$ yield, $95 \%$ ee

To a solution of 3a (38.9 mg, 1.0 equiv.) in $2 \mathrm{~mL} \mathrm{DCM}$ was added trifluoroacetic acid $(0.5 \mathrm{~mL})$, the reaction was stirred at room temperature for 1.5 hours and monitored by TLC. The solvent was evaporated under reduced pressure and the residue was redissolved in $2.0 \mathrm{~mL}$ DCM. TsCl $(21.0 \mathrm{mg})$ and pyridine $(10.0 \mu \mathrm{L})$ were added, subsequently. The mixture was stirred at room temperature for $2.5 \mathrm{~h}$ hours, and monitored by TLC. The mixture was purified by column chromatography on silica gel with petroleum ether/ethyl acetate (10:1) to obtain the product $8 \mathbf{a}(29.0 \mathrm{mg}, 65 \%$ yield, $95 \%$ ee) as white solid.

\section{Synthesis of $9 \mathrm{a}$}

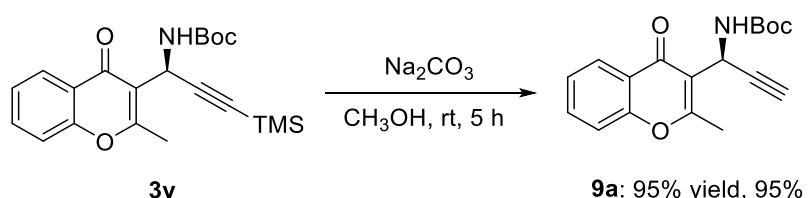

To a solution of $\mathbf{3 y}$ (420 mg, 1.0 equiv.) in 10.0 mL CH 3 OH was added $\mathrm{Na}_{2} \mathrm{CO}_{3}$ (231 $\mathrm{mg}$ ), the reaction was stirred at room temperature for 5 hours, and monitored by TLC. Then, the solvent was evaporated under reduced pressure and the residue was purified by column chromatography on silica gel with petroleum ether/ethyl acetate (10:1) to obtain the product 9a (327.2 mg, 95\% yield, 95\% ee) as a colorless oil.

\section{Synthesis of $9 b$}




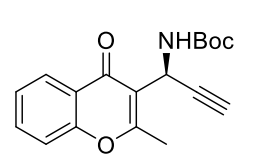

9 a

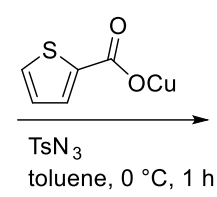

toluene, $0{ }^{\circ} \mathrm{C}, 1 \mathrm{~h}$

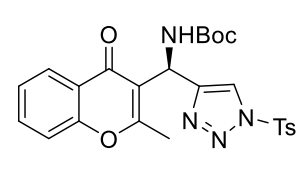

9b: $99 \%$ yield, $95 \%$ ee

To a solution of 9a (31.3 mg, $0.1 \mathrm{mmol}, 1.0$ equiv.) in $1.0 \mathrm{~mL}$ toluene was added $\mathrm{TsN}_{3}$ $(24.1 \mu \mathrm{L})$, copper(I) thiophene-2-carboxylate $(1.9 \mathrm{mg}, 10 \mathrm{~mol} \%)$. The reaction was stirred at $0{ }^{\circ} \mathrm{C}$ for 1 hour, and monitored by TLC. Then, the mixture was purified by column chromatography on silica gel with petroleum ether/ethyl acetate (10:1) to obtain the product $\mathbf{9 b}(55.4 \mathrm{mg}, 99 \%$ yield, $95 \%$ ee) as white solid.

\section{Synthesis of 9c}

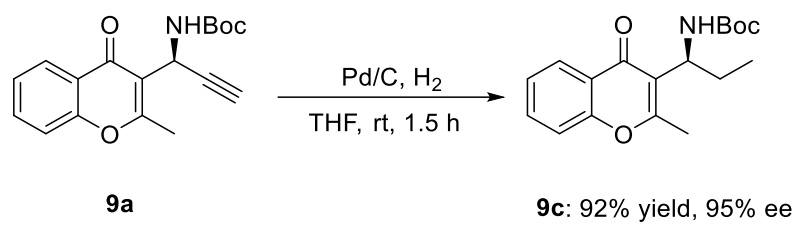

To a solution of 9a (31.3 mg, $0.1 \mathrm{mmol}, 1.0$ equiv.) in $3 \mathrm{~mL}$ THF was added Pd/C (5 mol\%). The reaction was stirred under the hydrogen atmosphere for 1.5 hours at room temperature, and monitored by TLC. After a simple filtration, the solvent was evaporated under reduced pressure and purified by column chromatography on silica gel with petroleum ether/ethyl acetate (10:1) to obtain the product 9c $(29.2 \mathrm{mg}, 92 \%$ yield, $95 \%$ ee) as colorless oil.

\section{The experimental procedure for synthesis of $2 \mathrm{z}$ and $5 \mathrm{a}$}

\section{Synthesis of $2 z$}<smiles>C#Cc1ccc2c(c1)CCC1C2CCC2C1CCC21OCCO1</smiles>

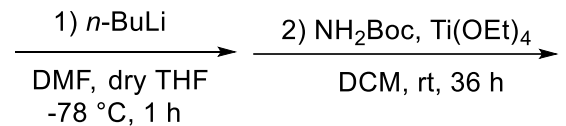

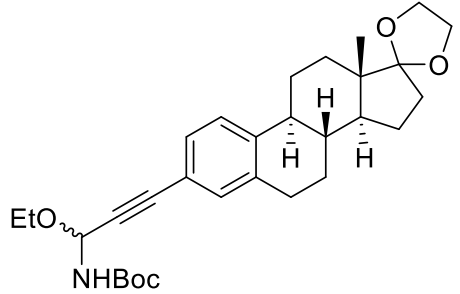

$2 z$

Compound $\mathbf{2 z}$ was prepared according to the literature. ${ }^{4,5}$

Step 1: Under an atmosphere of argon, to a solution of estrone-derived ethyne (1 mmol, $322.5 \mathrm{mg}, 1$ equiv.) dissolved in dry THF (5.0 mL) was added $n$-BuLi (1.1 equiv., $2.5 \mathrm{M}$ in n-hexane) over a period of 5 minutes at $-78{ }^{\circ} \mathrm{C}$, and the mixture was stirred at $-78{ }^{\circ} \mathrm{C}$ for 10 minutes, followed by the addition of DMF (2 equiv.) dropwise. The mixture was stirred at $-78^{\circ} \mathrm{C}$ for further 30 minutes, then allowed to warm to room temperature. The reaction was quenched by addition of saturated $\mathrm{NH}_{4} \mathrm{Cl}$ solution and extracted with EA. The combined organic layers were washed with saturated brine, dried over $\mathrm{Na}_{2} \mathrm{SO}_{4}$, and the solvent was evaporated under reduced 
pressure to afford the estrone-derived alkynyl aldehyde as yellow oil. The crude product was used in the next step without further purification.

Step 2: Under an atmosphere of argon, a solution of estrone-derived alkynyl aldehyde $^{4}$ (1 equiv.), tert-butyl carbamate (1.5 equiv.) and $\mathrm{Ti}(\mathrm{OEt})_{4}(1.5$ equiv.) in dichloromethane $(5.0 \mathrm{~mL})$ was stirred at room temperature for 36 hours. Then, the reaction was quenched with water, and the mixture was filtered through a pad of silica gel. The filtrate was extracted with DCM, and the organic layer was washed with saturated brine, dried over $\mathrm{Na}_{2} \mathrm{SO}_{4}$. Then, the solvent was evaporated under reduced pressure and purified by column chromatography on silica gel with petroleum ether/ethyl acetate (20:1 to $15: 1)$ to obtain the product $\mathbf{2 z}(108.3 \mathrm{mg}, 22 \%$ yield) as yellow oil.

Synthesis of 5 a

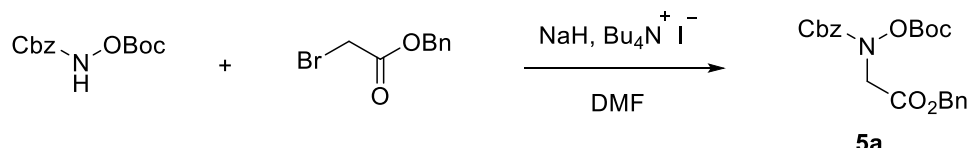

Compound 5a was prepared according to the literature. .,7 $^{6,}$

To a solution of benzyl ((tert-butoxycarbonyl)oxy)carbamate ${ }^{6}(23.8 \mathrm{mmol}, 1$ equiv.) in DMF (80 mL) was added $\mathrm{NaH}$ (60\%, 1.2 equiv.), benzyl 2-bromoacetate (1.2 equiv.), and tetrabutylammonium iodide ( 0.1 equiv.) at $0{ }^{\circ} \mathrm{C}$ under $\mathrm{N}_{2}$ atmosphere. The mixture was stirred at $0{ }^{\circ} \mathrm{C}$ for $12 \mathrm{~h}$ (monitored by TLC). Then, the reaction was quenched with aqueous sat. $\mathrm{NH}_{4} \mathrm{Cl}$ and extracted with ethyl acetate. The combined organic layer was washed with brine and dried over $\mathrm{Na}_{2} \mathrm{SO}_{4}$. The solvent was evaporated under reduced pressure and the residue was purified by flash chromatography on silica gel (petroleum ether/ethyl acetate 10:1) to obtain compound 5a as colorless oil (5.93 g, 60\% yield).

\section{X-ray crystal structure for 30 and $6 \mathrm{~h}$}

CCDC 2081446 (3o) contains the supplementary crystallographic data for this paper. These data can be obtained free of charge from The Cambridge Crystallographic Data Centre via www.ccdc.cam.ac.uk/data_request/cif.

The colorless crystal in flake-shape, with approximate dimensions of $0.070 \times 0.178 \times$ $0.482 \mathrm{~mm}^{3}$, was selected and mounted for the single-crystal X-ray diffraction. The data set was collected by Bruker D8 Venture Photon II diffractometer at 172(2)K equipped with micro-focus $\mathrm{Cu}$ radiation source $\left(K_{\alpha}=1.54178 \AA\right)$. Applied with face-indexed numerical absorption correction, the structure solution was solved and refinement was processed by SHELXTL (version 6.14) and OLEX 2.3 program package. ${ }^{8-11}$ The structure was analyzed by ADDSYM routine implemented in PLATON suite and no higher symmetry was suggested. ${ }^{12}$
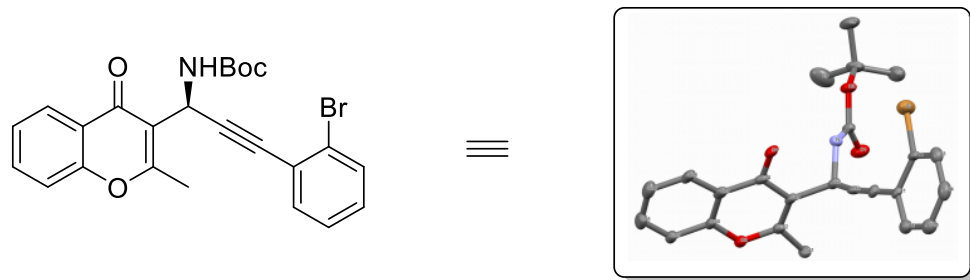

$30 \%$ ellipsoid probability 
Crystallographic Data for $\mathrm{C}_{24} \mathrm{H}_{22} \mathrm{BrNO}_{4}$

\begin{tabular}{|c|c|}
\hline Formula & $\mathrm{C}_{24} \mathrm{H}_{22} \mathrm{BrNO}_{4}$ \\
\hline Formula mass (amu) & 468.33 \\
\hline Space group & $\mathrm{P} 21$ \\
\hline$a(\AA)$ & $10.1658(10)$ \\
\hline$b(\AA)$ & $10.0421(9)$ \\
\hline$c(\AA)$ & $11.1308(11)$ \\
\hline$\alpha(\operatorname{deg})$ & 90 \\
\hline$\beta(\operatorname{deg})$ & $109.042(6)$ \\
\hline$\gamma(\operatorname{deg})$ & 90 \\
\hline$V\left(\AA^{3}\right)$ & $1074.12(18)$ \\
\hline$Z$ & 2 \\
\hline$\lambda(\AA)$ & 1.54178 \\
\hline$T(\mathrm{~K})$ & $172 \mathrm{~K}$ \\
\hline$\rho_{\text {calcd }}\left(\mathrm{g} \mathrm{cm}^{-3}\right)$ & 1.448 \\
\hline$\mu\left(\mathrm{mm}^{-1}\right)$ & 2.860 \\
\hline Transmission factors & $0.458-0.975$ \\
\hline $2 \theta_{\max }(\mathrm{deg})$ & 68.660 \\
\hline No. of unique data, including $F_{\mathrm{o}}^{2}<0$ & 3623 \\
\hline No. of unique data, with $F_{\mathrm{o}}^{2}>2 \sigma\left(F_{\mathrm{o}}^{2}\right)$ & 3135 \\
\hline No. of variables & 278 \\
\hline$R(F)$ for ${F_{\mathrm{o}}}^{2}>2 \sigma\left({F_{\mathrm{o}}}^{2}\right)^{a}$ & 0.1274 \\
\hline$R_{\mathrm{w}}\left(F_{\mathrm{o}}^{2}\right)^{b}$ & 0.3222 \\
\hline Goodness of fit & 1.365 \\
\hline Flack parameter & 0.13 \\
\hline
\end{tabular}

CCDC 2106423 (Racemic 6h) contains the supplementary crystallographic data for this paper. These data can be obtained free of charge from The Cambridge Crystallographic Data Centre via www.ccdc.cam.ac.uk/data_request/cif. The colorless crystal in cube-shape, with approximate dimensions of $0.119 \times 0.149 \times$ $0.183 \mathrm{~mm}^{3}$, was selected and mounted for the single-crystal X-ray diffraction. The data set was collected by Bruker D8 Venture Photon II diffractometer at 172(2)K equipped with micro-focus $\mathrm{Cu}$ radiation source $\left(K_{\alpha}=1.54178 \AA\right)$. Applied with face-indexed numerical absorption correction, the structure solution was solved and 
refinement was processed by SHELXTL (version 6.14) and OLEX 2.3 program package..$^{8-11}$ The structure was analyzed by ADDSYM routine implemented in PLATON suite and no higher symmetry was suggested. ${ }^{12}$

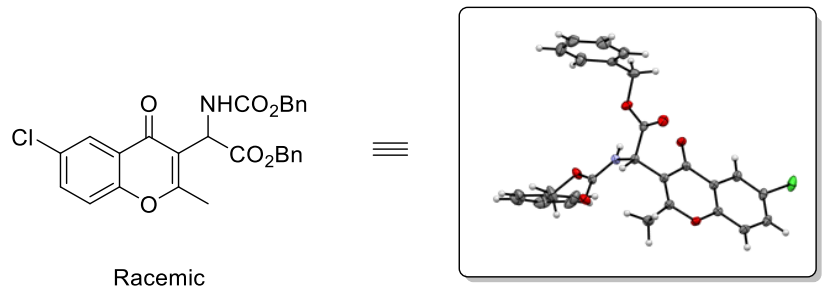

$30 \%$ ellipsoid probability

Crystallographic Data for $\mathrm{C}_{27} \mathrm{H}_{22} \mathrm{ClNO}_{6}$

\begin{tabular}{|c|c|}
\hline Formula & $\mathrm{C}_{27} \mathrm{H}_{22} \mathrm{ClNO}_{6}$ \\
\hline Formula mass (amu) & 491.90 \\
\hline Space group & $\mathrm{P}-1$ \\
\hline$a(\AA)$ & $10.2296(3)$ \\
\hline$b(\AA)$ & $11.1415(4)$ \\
\hline$c(\AA)$ & $11.1911(4)$ \\
\hline$\alpha(\mathrm{deg})$ & $104.557(1)$ \\
\hline$\beta(\mathrm{deg})$ & $98.068(1)$ \\
\hline$\gamma(\operatorname{deg})$ & $98.641(1)$ \\
\hline$V\left(\AA^{3}\right)$ & $1199.20(7)$ \\
\hline$Z$ & 2 \\
\hline$\lambda(\AA)$ & 1.54178 \\
\hline$T(\mathrm{~K})$ & $172 \mathrm{~K}$ \\
\hline$\rho_{\text {calcd }}\left(\mathrm{g} \mathrm{cm}^{-3}\right)$ & 1.362 \\
\hline$\mu\left(\mathrm{mm}^{-1}\right)$ & 1.781 \\
\hline Transmission factors & $0.748-0.894$ \\
\hline $2 \theta_{\max }(\mathrm{deg})$ & 68.348 \\
\hline No. of unique data, including $F_{\mathrm{o}}{ }^{2}<0$ & 4318 \\
\hline No. of unique data, with $F_{\mathrm{o}}^{2}>2 \sigma\left({F_{\mathrm{o}}}^{2}\right)$ & 4054 \\
\hline No. of variables & 321 \\
\hline$R(F)$ for $F_{\mathrm{o}}^{2}>2 \sigma\left(F_{\mathrm{o}}^{2}\right)^{a}$ & 0.0386 \\
\hline$R_{\mathrm{w}}\left(F_{\mathrm{o}}^{2}\right)^{b}$ & 0.1108 \\
\hline Goodness of fit & 1.022 \\
\hline
\end{tabular}




\section{ECD calculations of $6 \mathbf{j}$}

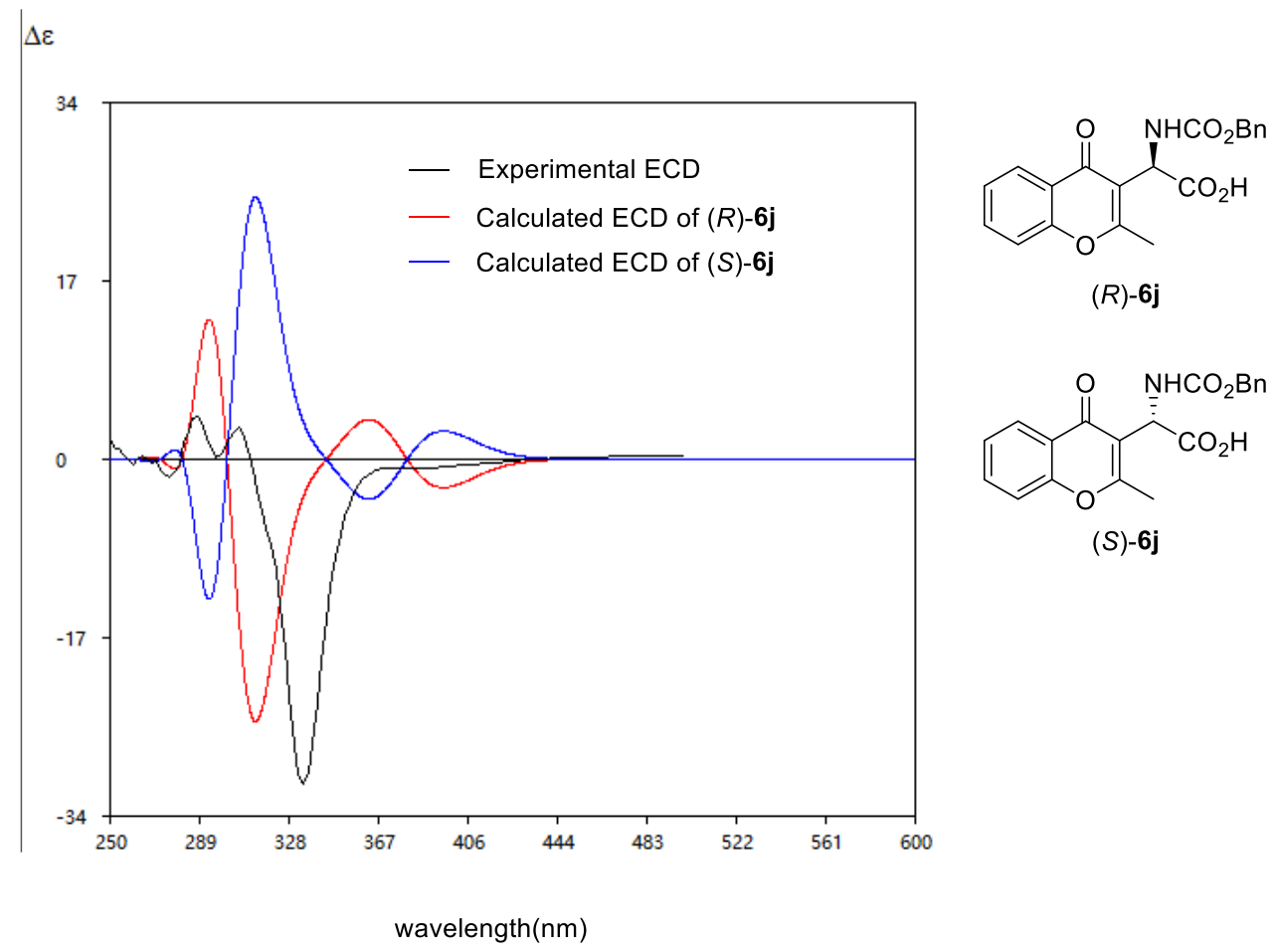

Conformational searches of $\mathbf{6 j}$ were carried out with CONFLEX using the Merck molecular force field (MMFF) with standard parameters. ${ }^{13}$ Next, they were subjected to geometry optimization at the B3LYP/6-31G(d) level by DFT calculations. Based on the DFT energies, conformers with a Boltzmann distribution $\geq 1 \%$ were chosen. The conformers were then imported into Gaussian 09 package. ${ }^{14}$ The ECD curve of each conformer was calculated by the TDDFT method at the B3LYP/6-311++G (d, p) level with the CPCM model in a MeOH solution. The final ECD curve was summed based on the Boltzmann averaging of all the ECD curves from all the conformers. ${ }^{13,14}$

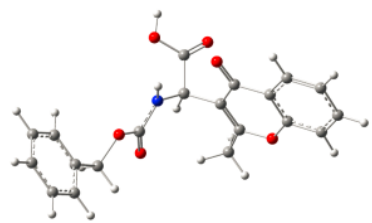

$1 \mathrm{C} 1$

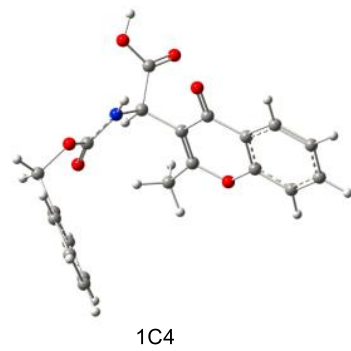

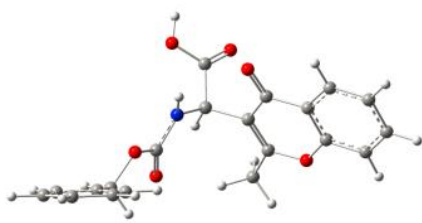

$1 \mathrm{C} 2$

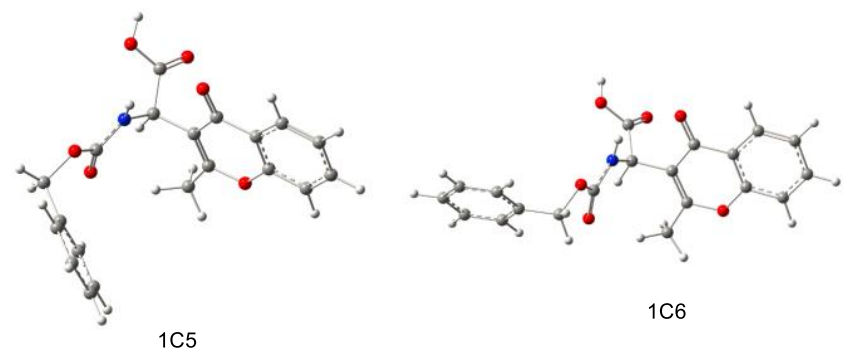




\section{Reference}

1. Han, J.; Wang, T.; Feng, S.; Li, C.; Zhang, Z. One-pot Synthesis of 3-(Furan-2-yl)-4H-Chromen-4-ones from 1-(2-Hydroxyphenyl)butane-1,3-Diones and 2,5-Dimethoxy-2,5-Dihydrofuran Catalyzed via K10 Montmorillonite under Solvent-Free Conditions. Green. Chemistry. 2016, 18, 4092-4097.

2. Silva, A. M. S.; Pinto, D. C. G. A.; Cavaleiro, J. A. S.; Levai, Albert.; Patonay, T. Synthesis and Reactivity of Styrylchromones. Arkivoc. 2004 (vii) 106-123.

3. Gomes, A.; Freitas, M.; Fernandes, E,; Lima, J. L. F. C. Biological Activities of 2-Styrylchromones. Mini. Rev. Med. Chem. 2010, 10, 1-7.

4. Wang, Y.; Mo, M.; Zhu, K.; Zheng, C.; Zhang, H.; Wang, W.; Shao, Z. Asymmetric Synthesis of syn-Propargylamines and Unsaturated $\beta$-Amino Acids under Bronsted Base Catalysis. Nat. Commun. 2015, 6, 8544-8552.

5. Hack, D.; Chauhan, P.; Deckers, K.; Hermann, G. N.; Mertens, L.; Raabe, G.; Enders, D. Combining Silver Catalysis and Organocatalysis: A Sequential Michael Addition/Hydroalkoxylation One-pot Approach to Annulated Coumarins. Org. Lett. 2014, 16, 5188-5191.

6. Liu, Y. Y.; Duan, S. W.; Zhang, R.; Liu, Y. H.; Chen, J. R.; Xiao, W. J. Base-Catalyzed Controllable Reaction of 3-Ylideneoxindoles with O-Boc Hydroxycarbamates for the Synthesis of Amidoacrylates and Apiroaziridine Oxindoles. Org. Biomol. Chem. 2016, 14, 5224-5228.

7. Fukahori, Y.; Takayama, Y.; Imaoka, T.; Iwamoto, O.; Nagasawa, K. Intramolecular 1,3-Dipolar Cycloaddition-Mediated Stereoselective Synthesis of Disubstituted Cyclopentane: A Simple Model for the Cyclopentane Ring System of Polycyclic Oroidine Alkaloids. Chem. Asian. J. 2013, 8, 244-250.

8. Sheldrick, G. M. Acta. Cryst. 2008, A64, 112-122.

9. Sheldrick, G. M. Acta. Cryst. 2015, A71, 3-8.

10. Sheldrick, G. M. Acta. Cryst. 2015, C71, 3-8.

11. Dolomanov, O.V., Bourhis, L.J., Gildea, R.J., Howard, J. A. K., Puschmann, H. J. Appl. Cryst. 2009, 42, 339-341.

12. Spek, A. L. J. Appl. Cryst. 2003, 36, 7-13.

13. Jagannadh, B.; Reddy, S. S.; Thangavelu, R. P. J. Mol. Model. 2004, 10, 55.

14. Gaussian 09; Revision D.01; Frisch, M. J.; Trucks, G. W.; Schlegel, H. B.; Scuseria, G. E.; Robb, M. A.; Cheeseman, J. R.; Scalmani, G.; Barone, V.; Mennucci, B.; Petersson, G. A.; Nakatsuji, H.; Caricato, M.; Li, X.; Hratchian, H. P.; Izmaylov, A. F.; Bloino, J.; Zheng, G.; Sonnenberg, J. L.; Hada, M.; Ehara, M.; Toyota, K.; Fukuda, R.; Hasegawa, J.; Ishida, M.; Nakajima, T.; Honda, Y.; Kitao, O.; Nakai, H.; Vreven, T.; Montgomery, J. A.; Jr.; Peralta, J. E.; Ogliaro, F.; Bearpark, M.; Heyd, J. J.; Brothers, E.; Kudin, K. N.; Staroverov, V. N.; Keith, T.; Kobayashi, R.; Normand, J.; Raghavachari, K.; Rendell, A.; Burant, J. C.; Iyengar, S. S.; Tomasi, J.; Cossi, M.; Rega, N.; Millam, J. M.; Klene, M.; Knox, J. E.; Cross, J. B.; Bakken, V.; Adamo, C.; Jaramillo, J.; Gomperts, R.; Stratmann, R. E.; Yazyev, O.; Austin, A. J.; Cammi, R.; Pomelli, C.; Ochterski, J. W.; Martin, R. L.; Morokuma, K.; Zakrzewski, V. G.; Voth, G. A.; Salvador, P.; Dannenberg, J. J.; 
Dapprich, S.; Daniels, A. D.; Farkas, O.; Foresman, J. B.; Ortiz, J. V.; Cioslowski, J.; Fox,D. J.; Gaussian, Inc., Wallingford CT, 2013.

\section{The analytical and spectral characterization data for the products}

tert-Butyl (S)-(1-(2-methyl-4-oxo-4H-chromen-3-yl)-3-phenylprop-2-yn-1-yl)carbamate (3a)<smiles>Cc1oc2ccccc2c(=O)c1[C@H](C#Cc1ccccc1)C(=O)OC(C)(C)C</smiles>

Compound 3a: Prepared in $0.2 \mathrm{mmol}$ scale using 1.5 equiv of $2 \mathbf{a}$ at $70{ }^{\circ} \mathrm{C}$ for 63 h. $90 \%$ yield $(70.0 \mathrm{mg})$, colorless oil, 95\% ee. HPLC (chiral ID column), hexane $/ i-\mathrm{PrOH}=70 / 30$, flow rate $0.8 \mathrm{ml} / \mathrm{min}, \lambda=254 \mathrm{~nm}, t \mathrm{r}$ (minor) $=8.79 \mathrm{~min}, t \mathrm{r}$

$($ major $)=13.73 \mathrm{~min}$.

$[\alpha]^{25}=+64.2\left(\mathrm{c}=1.0\right.$ in $\left.\mathrm{CH}_{2} \mathrm{Cl}_{2}\right)$.

${ }^{1} \mathrm{H}$ NMR (400 MHz, $\left.\mathrm{CDCl}_{3}\right) \delta 8.20(\mathrm{~d}, J=7.8 \mathrm{~Hz}, 1 \mathrm{H}), 7.64(\mathrm{t}, J=7.6 \mathrm{~Hz}, 1 \mathrm{H}), 7.46$ $-7.35(\mathrm{~m}, 4 \mathrm{H}), 7.28-7.21(\mathrm{~m}, 3 \mathrm{H}), 6.55(\mathrm{~d}, J=9.0 \mathrm{~Hz}, 1 \mathrm{H}), 5.88(\mathrm{~d}, J=9.2 \mathrm{~Hz}, 1 \mathrm{H})$, $2.65(\mathrm{~s}, 3 \mathrm{H}), 1.43(\mathrm{~s}, 9 \mathrm{H}) .{ }^{13} \mathrm{C}\{1 \mathrm{H}\} \mathrm{NMR}\left(100 \mathrm{MHz}, \mathrm{CDCl}_{3}\right) \delta$ 177.0, 163.4, 156.0, 155.2, 133.7, 132.0, 128.3, 128.2, 125.8, 125.1, 123.7, 122.8, 119.6, 117.9, 86.9, 82.1, 80.0, 41.0, 28.5, 18.6. HRMS (FTMS-ESI), m/z calcd for $\mathrm{C}_{24} \mathrm{H}_{23} \mathrm{NNaO}_{4}{ }^{+}\left([\mathrm{M}+\mathrm{Na}]^{+}\right)$ 412.1519 , found 412.1518 .

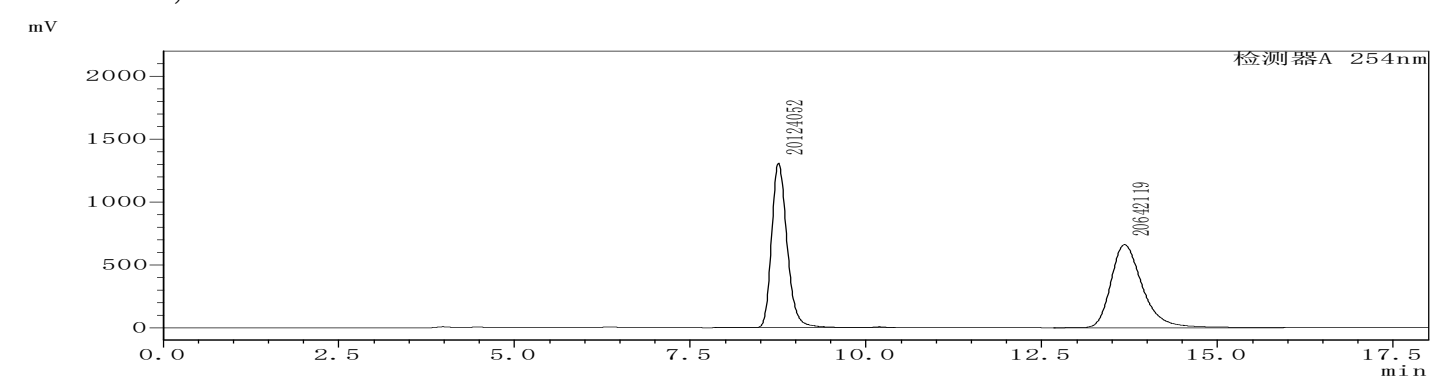

racemic

\begin{tabular}{|c|c|c|c|}
\hline & Retention Time & Area & \%Area \\
\hline 1 & 8.756 & 20124052 & 49.365 \\
\hline 2 & 13.679 & 20642119 & 50.635 \\
\hline
\end{tabular}

$\mathrm{mV}$

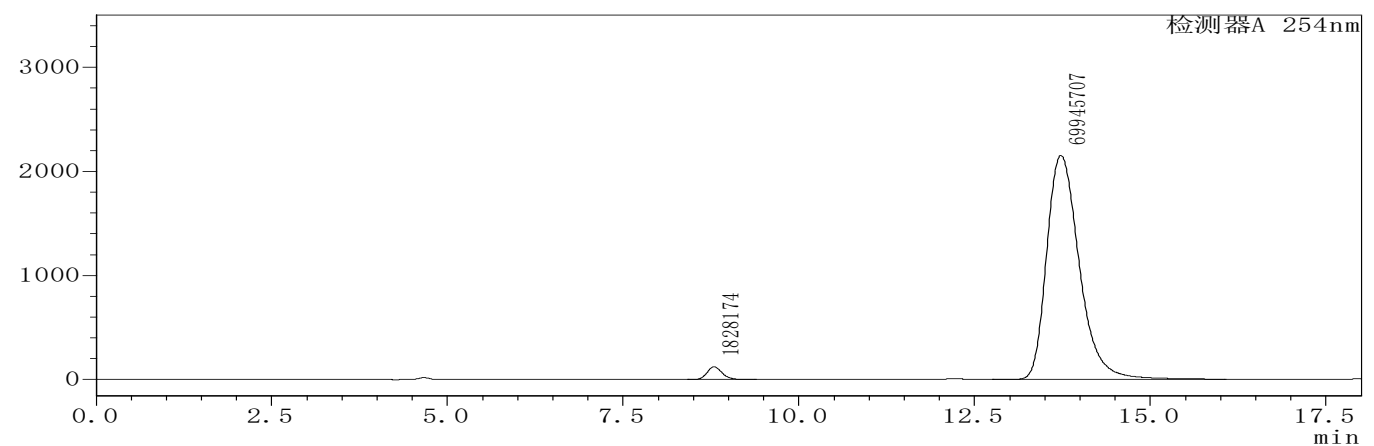

enantio-enriched

\begin{tabular}{|c|c|c|c|}
\hline & Retention Time & Area & \%Area \\
\hline 1 & 8.793 & 1828174 & 2.547 \\
\hline 2 & 13.728 & 69945707 & 97.453 \\
\hline
\end{tabular}


tert-Butyl (S)-(1-(2,6-dimethyl-4-oxo-4H-chromen-3-yl)-3-phenylprop-2-yn-1-yl)carbamate (3b)<smiles>Cc1ccc2oc(C)c(C(C#CPc3ccccc3)NC(=O)OCc3ccccc3)c(=O)c2c1</smiles>

Compound 3b: Prepared in $0.2 \mathrm{mmol}$ scale using 1.5 equiv of $2 \mathbf{a}$ at $70{ }^{\circ} \mathrm{C}$ for $60 \mathrm{~h} .77 \%$ yield $(62.0 \mathrm{mg})$, colorless oil, 95\% ee. HPLC (chiral ID column), hexane $/ i-\mathrm{PrOH}=70 / 30$, flow rate $0.8 \mathrm{ml} / \mathrm{min}, \lambda=254 \mathrm{~nm}$, $\operatorname{tr}($ minor $)=8.58 \mathrm{~min}, \mathrm{tr}$ (major) $=11.51 \mathrm{~min}$.

$[\alpha]^{25} \mathrm{D}=+79.6\left(\mathrm{c}=1.0\right.$ in $\left.\mathrm{CH}_{2} \mathrm{Cl}_{2}\right)$.

${ }^{1} \mathrm{H}$ NMR $\left(400 \mathrm{MHz}, \mathrm{CDCl}_{3}\right) \delta 7.98(\mathrm{~s}, 1 \mathrm{H}), 7.45(\mathrm{~d}, J=8.6 \mathrm{~Hz}, 1 \mathrm{H}), 7.42-7.37(\mathrm{~m}$, $2 \mathrm{H}), 7.31(\mathrm{~d}, J=8.6 \mathrm{~Hz}, 1 \mathrm{H}), 7.26-7.19(\mathrm{~m}, 3 \mathrm{H}), 6.57(\mathrm{~d}, J=8.8 \mathrm{~Hz}, 1 \mathrm{H}), 5.86(\mathrm{~d}, J$ $=9.2 \mathrm{~Hz}, 1 \mathrm{H}), 2.64(\mathrm{~s}, 3 \mathrm{H}), 2.44(\mathrm{~s}, 3 \mathrm{H}), 1.43(\mathrm{~s}, 9 \mathrm{H}) .{ }^{13} \mathrm{C}\{1 \mathrm{H}\} \mathrm{NMR}(100 \mathrm{MHz}$, $\left.\mathrm{CDCl}_{3}\right) \delta 177.2,163.3,155.2,154.2,135.1,135.0,132.0,128.3,128.2,125.1,123.3$, 122.8, 119.4, 117.6, 87.0, 82.0, 80.0, 41.1, 28.5, 21.1, 18.6. HRMS (FTMS-ESI), m/z calcd for $\mathrm{C}_{25} \mathrm{H}_{25} \mathrm{NNaO}_{4}{ }^{+}\left([\mathrm{M}+\mathrm{Na}]^{+}\right) 426.1676$, found 426.1676 .

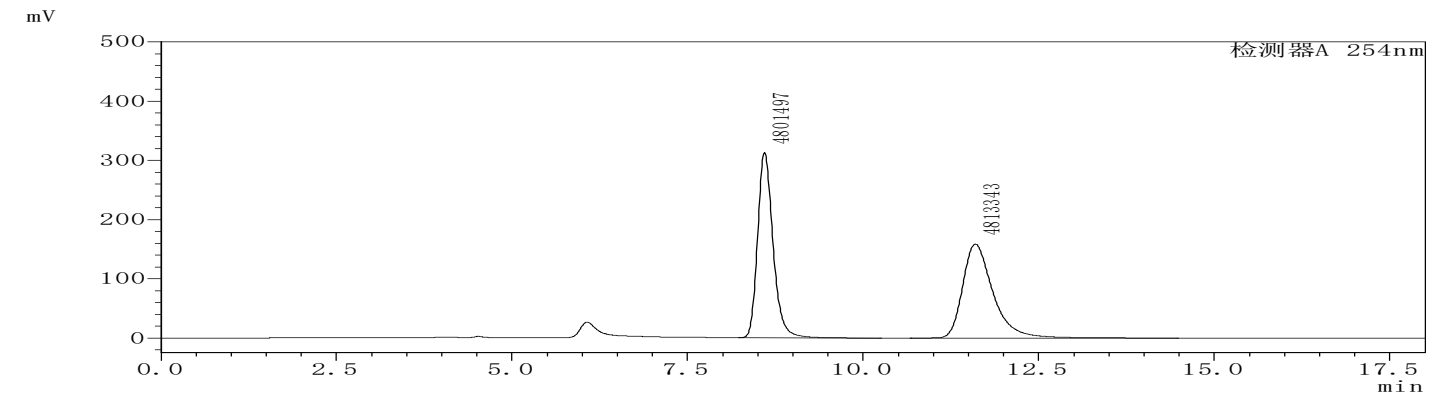

racemic

\begin{tabular}{|c|c|c|c|}
\hline & Retention Time & Area & \%Area \\
\hline 1 & 8.595 & 4801497 & 49.938 \\
\hline 2 & 11.597 & 4813343 & 50.062 \\
\hline
\end{tabular}

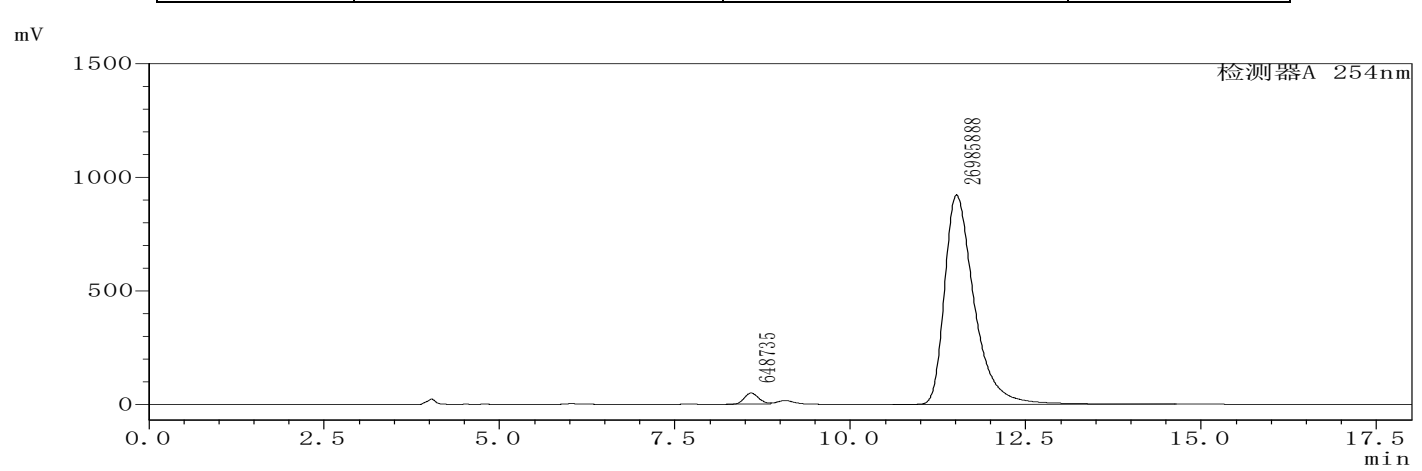

enantio-enriched

\begin{tabular}{|c|c|c|c|}
\hline & Retention Time & Area & \%Area \\
\hline 1 & 8.584 & 648735 & 2.348 \\
\hline 2 & 11.514 & 26985888 & 97.652 \\
\hline
\end{tabular}


tert-Butyl (S)-(1-(6-chloro-2-methyl-4-oxo-4H-chromen-3-yl)-3-phenylprop-2-

yn-1-yl)carbamate (3c)<smiles>Cc1oc2ccc(Cl)cc2c(=O)c1C(C#Cc1ccccc1)NC(=O)OC(C)(C)C</smiles>

Compound 3c: Prepared in $0.2 \mathrm{mmol}$ scale using 1.5 equiv of $2 \mathbf{a}$ at $70{ }^{\circ} \mathrm{C}$ for $65 \mathrm{~h} .86 \%$ yield $(73.3 \mathrm{mg})$, colorless oil, 95\% ee. HPLC (chiral ID column), hexane $/ i-\mathrm{PrOH}=70 / 30$, flow rate $0.8 \mathrm{ml} / \mathrm{min}, \lambda=254$ $\mathrm{nm}, t \mathrm{r}($ minor $)=8.98 \mathrm{~min}, t \mathrm{r}$ (major) $=13.10 \mathrm{~min}$.

$[\alpha]^{25} \mathrm{D}=+98.2\left(\mathrm{c}=1.0\right.$ in $\left.\mathrm{CH}_{2} \mathrm{Cl}_{2}\right)$.

${ }^{1} \mathrm{H}$ NMR $\left(400 \mathrm{MHz}, \mathrm{CDCl}_{3}\right) \delta 8.16(\mathrm{~s}, 1 \mathrm{H}), 7.58(\mathrm{~d}, J=8.2 \mathrm{~Hz}, 1 \mathrm{H}), 7.41-7.36(\mathrm{~m}$, $3 \mathrm{H}), 7.28-7.23(\mathrm{~m}, 3 \mathrm{H}), 6.45(\mathrm{~d}, J=8.4 \mathrm{~Hz}, 1 \mathrm{H}), 5.87(\mathrm{~d}, J=9.2 \mathrm{~Hz}, 1 \mathrm{H}), 2.66(\mathrm{~s}$, $3 \mathrm{H}), 1.44(\mathrm{~s}, 9 \mathrm{H}) .{ }^{13} \mathrm{C}\{1 \mathrm{H}\} \mathrm{NMR}\left(125 \mathrm{MHz}, \mathrm{CDCl}_{3}\right) \delta 175.8,163.7,155.1,154.2$, 133.9, 132.0, 131.0, 128.4, 128.2, 125.2, 124.5, 122.6, 119.8, 119.6, 86.5, 82.3, 80.1, $40.9,28.5,18.6$. HRMS (FTMS-ESI), m/z calcd for $\mathrm{C}_{24} \mathrm{H}_{22} \mathrm{ClNNaO}_{4}{ }^{+}\left([\mathrm{M}+\mathrm{Na}]^{+}\right)$ $446.1130\left(448.1100\right.$ for $\left.{ }^{37} \mathrm{Cl}\right)$, found $446.1133\left(448.1103\right.$ for $\left.{ }^{37} \mathrm{Cl}\right)$.

$\mathrm{mV}$

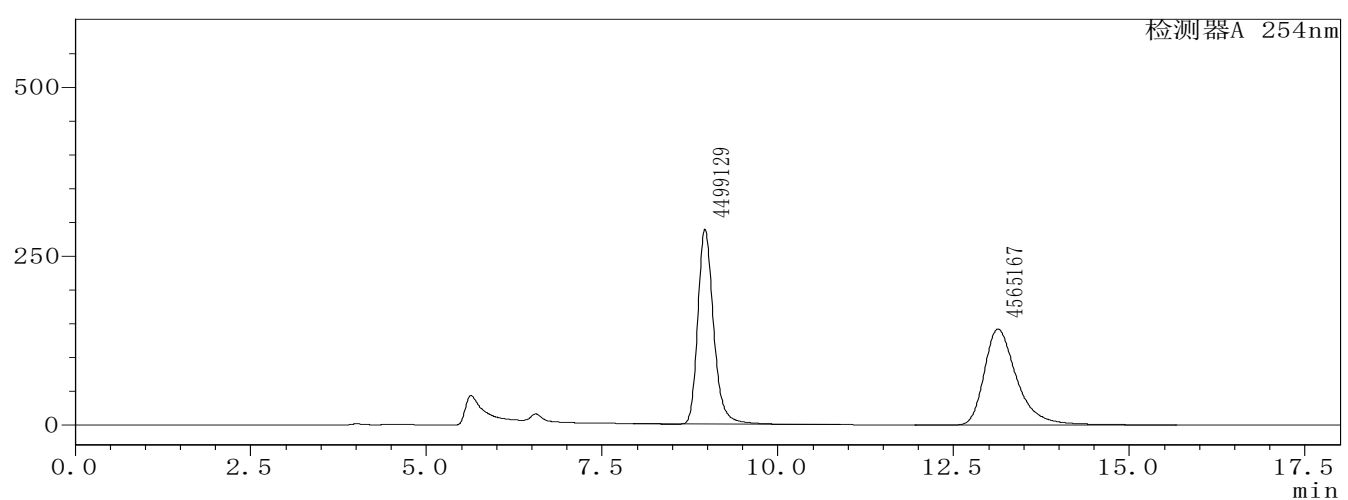

racemic

\begin{tabular}{|c|c|c|c|}
\hline & Retention Time & Area & \%Area \\
\hline 1 & 8.962 & 4499129 & 49.636 \\
\hline 2 & 13.132 & 4565167 & 50.364 \\
\hline
\end{tabular}

$\mathrm{mV}$

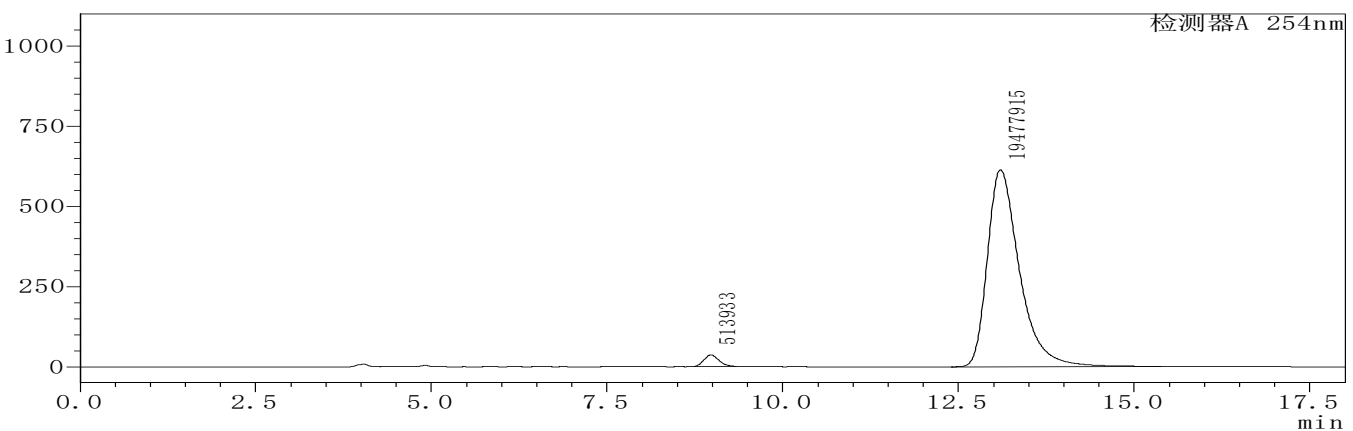

enantio-enriched

\begin{tabular}{|c|c|c|c|}
\hline & Retention Time & Area & \%Area \\
\hline 1 & 8.976 & 513933 & 2.571 \\
\hline 2 & 13.098 & 19477915 & 97.429 \\
\hline
\end{tabular}


tert-Butyl (S)-(1-(6-bromo-2-methyl-4-oxo-4H-chromen-3-yl)-3-phenylprop-2-

yn-1-yl)carbamate (3d)<smiles>Cc1oc2ccc(Br)cc2c(=O)c1[C@H](C#Cc1ccccc1)NC(=O)OCc1ccccc1</smiles>

Compound 3d: Prepared in $0.2 \mathrm{mmol}$ scale using 1.5 equiv of $2 \mathbf{a}$ at $70{ }^{\circ} \mathrm{C}$ for $60 \mathrm{~h} .80 \%$ yield $(74.7 \mathrm{mg})$, colorless oil, $96 \%$ ee. HPLC (chiral ID column), hexane $/ i-\mathrm{PrOH}=70 / 30$, flow rate $0.8 \mathrm{ml} / \mathrm{min}, \lambda=254$

$\mathrm{nm}, t \mathrm{r}($ minor $)=9.53 \mathrm{~min}, t \mathrm{r}$ (major) $=13.70 \mathrm{~min}$.

$[\alpha]^{25}=+99.4\left(\mathrm{c}=1.0\right.$ in $\left.\mathrm{CH}_{2} \mathrm{Cl}_{2}\right)$.

${ }^{1} \mathrm{H}$ NMR $\left(400 \mathrm{MHz}, \mathrm{CDCl}_{3}\right) \delta 8.31(\mathrm{~s}, 1 \mathrm{H}), 7.71(\mathrm{~d}, J=8.8 \mathrm{~Hz}, 1 \mathrm{H}), 7.43-7.36(\mathrm{~m}$, $2 \mathrm{H}), 7.30(\mathrm{~d}, J=9.0 \mathrm{~Hz}, 1 \mathrm{H}), 7.27-7.20(\mathrm{~m}, 3 \mathrm{H}), 6.44(\mathrm{~d}, J=8.8 \mathrm{~Hz}, 1 \mathrm{H}), 5.86(\mathrm{~d}, J$ $=9.2 \mathrm{~Hz}, 1 \mathrm{H}), 2.65(\mathrm{~s}, 3 \mathrm{H}), 1.43(\mathrm{~s}, 9 \mathrm{H}) .{ }^{13} \mathrm{C}\{1 \mathrm{H}\} \mathrm{NMR}\left(100 \mathrm{MHz}, \mathrm{CDCl}_{3}\right) \delta 175.7$, $163.7,155.1,154.7,136.7,132.0,128.5,128.4,128.2,124.9,122.6,119.9,118.5$, $86.4, \quad 82.3, \quad 80.1, \quad 40.9, \quad 28.5, \quad$ 18.6. HRMS (FTMS-ESI), $\mathrm{m} / \mathrm{z}$ calcd for $\mathrm{C}_{24} \mathrm{H}_{22} \mathrm{BrNNaO}_{4}{ }^{+}\left([\mathrm{M}+\mathrm{Na}]^{+}\right) 490.0624$ (492.0604 for $\left.{ }^{81} \mathrm{Br}\right)$, found 490.0630 (492.0610 for ${ }^{81} \mathrm{Br}$ ).

$\mathrm{mV}$

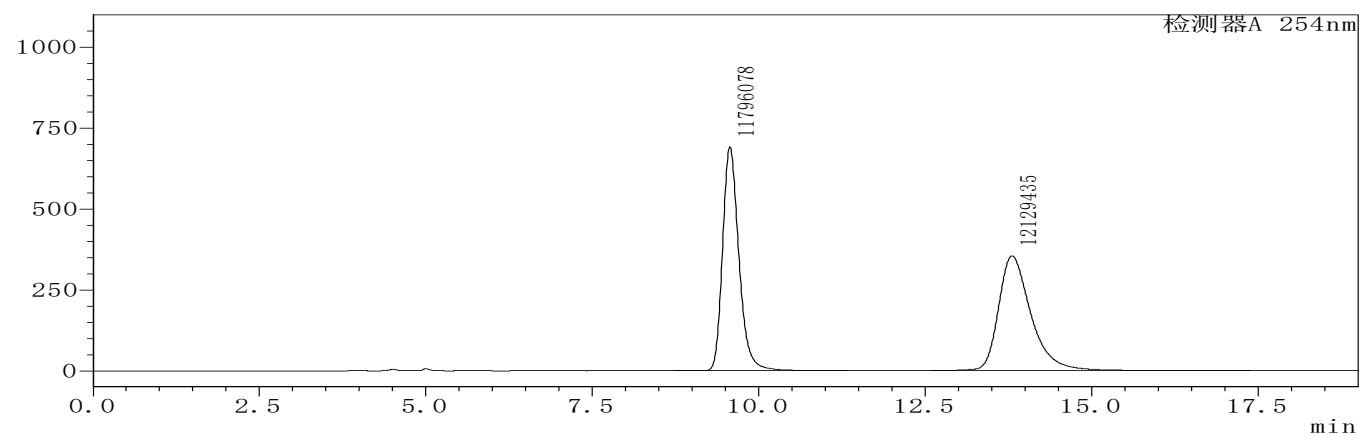

racemic

\begin{tabular}{|c|c|c|c|}
\hline & Retention Time & Area & \%Area \\
\hline 1 & 9.567 & 11796078 & 49.303 \\
\hline 2 & 13.807 & 12129435 & 50.697 \\
\hline
\end{tabular}

$\mathrm{mV}$

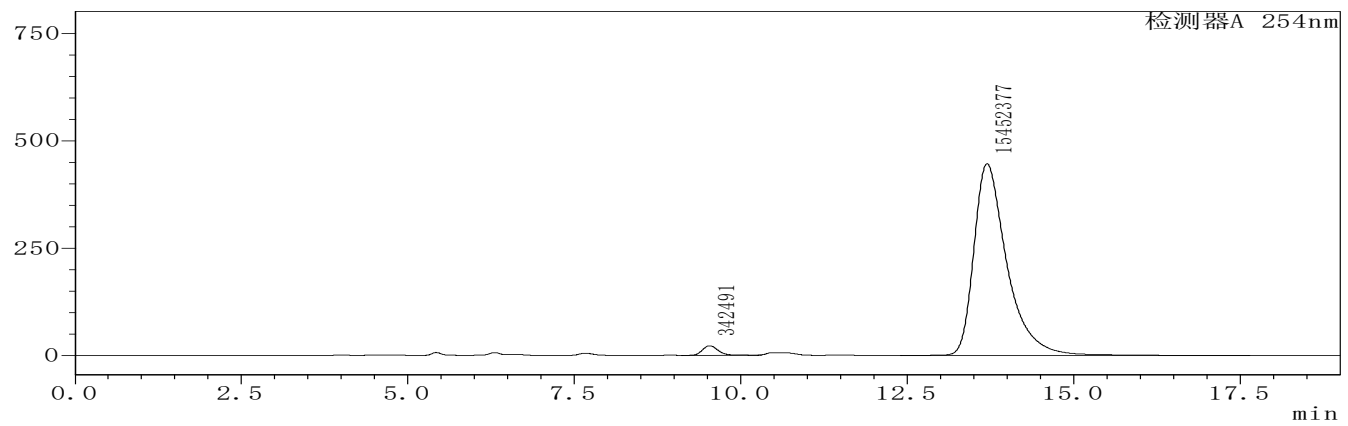

enantio-enriched

\begin{tabular}{|c|c|c|c|}
\hline & Retention Time & Area & \%Area \\
\hline 1 & 9.534 & 342491 & 2.168 \\
\hline 2 & 13.702 & 15452377 & 97.832 \\
\hline
\end{tabular}


tert-Butyl (S)-(1-(7-bromo-2-methyl-4-oxo-4H-chromen-3-yl)-3-phenylprop-2yn-1-yl)carbamate (3e)<smiles>Cc1oc2cc(Br)ccc2c(=O)c1[C@H](C#Cc1ccccc1)NC(=O)OC(C)(C)C</smiles>

Compound 3e: Prepared in $0.2 \mathrm{mmol}$ scale using 1.5 equiv of $2 \mathbf{a}$ at $70{ }^{\circ} \mathrm{C}$ for $60 \mathrm{~h} .79 \%$ yield $(73.6 \mathrm{mg})$, colorless oil, 95\% ee. HPLC (chiral ID column), hexane $/ i-\mathrm{PrOH}=70 / 30$, flow rate $0.8 \mathrm{ml} / \mathrm{min}, \lambda=254$ $\mathrm{nm}, t \mathrm{r}($ minor $)=8.68 \mathrm{~min}, t \mathrm{r}$ (major) $=13.13 \mathrm{~min}$.

$[\alpha]^{25}=+16.6\left(\mathrm{c}=1.0\right.$ in $\left.\mathrm{CH}_{2} \mathrm{Cl}_{2}\right)$.

${ }^{1} \mathrm{H}$ NMR $\left(400 \mathrm{MHz}, \mathrm{CDCl}_{3}\right) \delta 8.04(\mathrm{~d}, J=8.6 \mathrm{~Hz}, 1 \mathrm{H}), 7.59(\mathrm{~s}, 1 \mathrm{H}), 7.48(\mathrm{~d}, J=7.8$ $\mathrm{Hz}, 1 \mathrm{H}), 7.42-7.37(\mathrm{~m}, 2 \mathrm{H}), 7.29-7.20(\mathrm{~m}, 3 \mathrm{H}), 6.45(\mathrm{~d}, J=9.0 \mathrm{~Hz}, 1 \mathrm{H}), 5.86(\mathrm{~d}, J$ $=9.2 \mathrm{~Hz}, 1 \mathrm{H}), 2.64(\mathrm{~s}, 3 \mathrm{H}), 1.43(\mathrm{~s}, 9 \mathrm{H}) .{ }^{13} \mathrm{C}\{1 \mathrm{H}\} \mathrm{NMR}\left(100 \mathrm{MHz}, \mathrm{CDCl}_{3}\right) \delta 176.3$, $163.6,155.9,155.1,132.0,128.7,128.4,128.2$, 128.0, 127.3, 122.6, 122.5, 121.0, 120.0, 86.5, 82.3, 80.1, 40.9, 28.5, 18.6. HRMS (FTMS-ESI), m/z calcd for $\mathrm{C}_{24} \mathrm{H}_{22} \mathrm{BrNNaO}_{4}{ }^{+}\left([\mathrm{M}+\mathrm{Na}]^{+}\right) 490.0624\left(492.0604\right.$ for $\left.{ }^{81} \mathrm{Br}\right)$, found 490.0632 (492.0609 for ${ }^{81} \mathrm{Br}$ ).

$\mathrm{mV}$

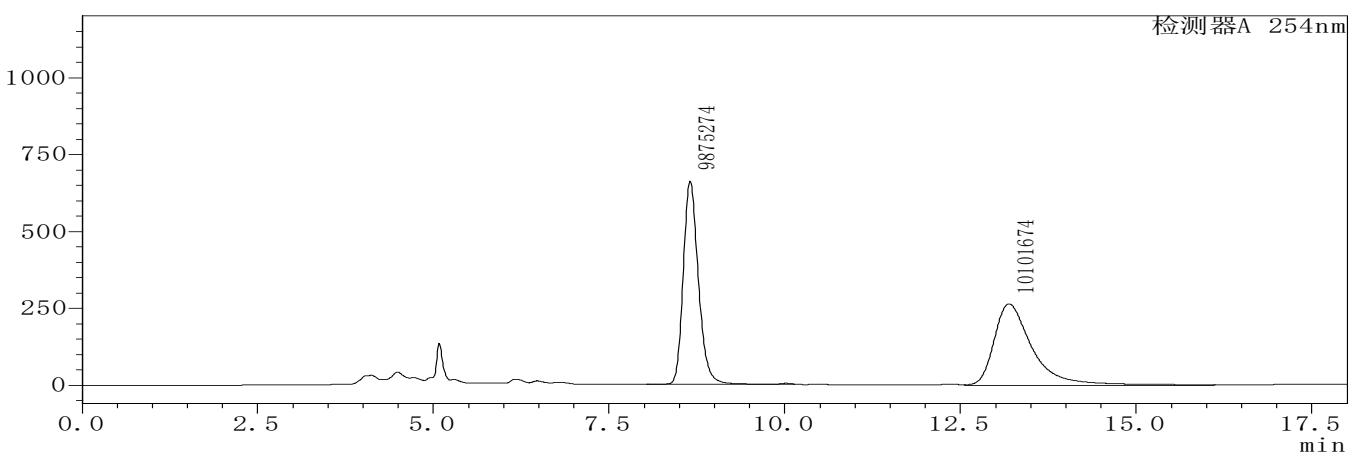

racemic

\begin{tabular}{|c|c|c|c|}
\hline & Retention Time & Area & \%Area \\
\hline 1 & 8.652 & 9875274 & 49.433 \\
\hline 2 & 13.190 & 10101674 & 50.567 \\
\hline
\end{tabular}

$\mathrm{mV}$

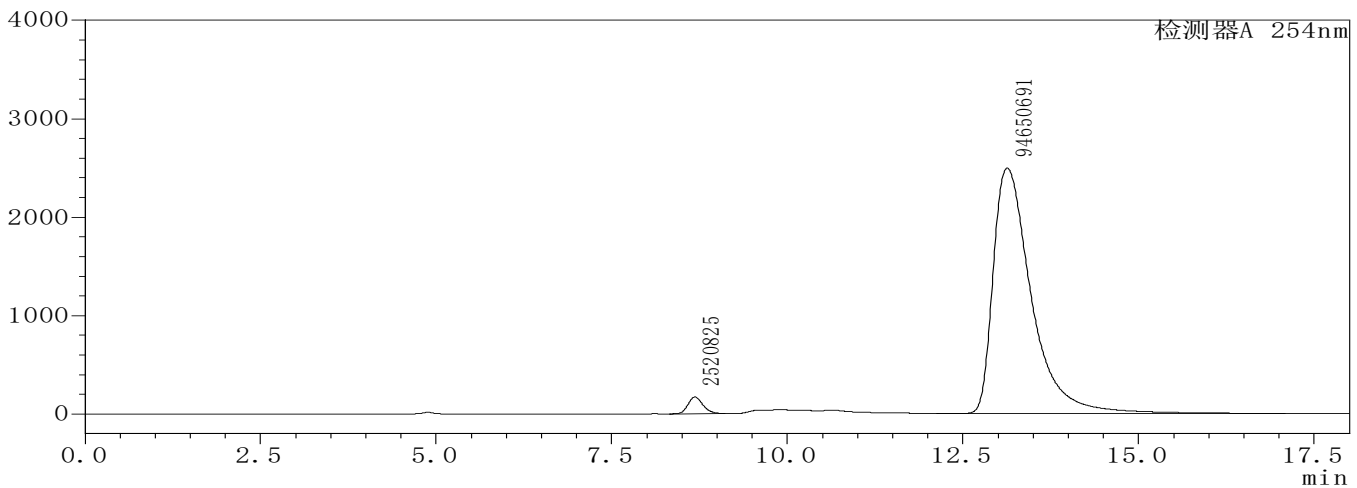

enantio-enriched

\begin{tabular}{|c|c|c|c|}
\hline & Retention Time & Area & \%Area \\
\hline 1 & 8.683 & 2520825 & 2.594 \\
\hline 2 & 13.130 & 94650691 & 97.406 \\
\hline
\end{tabular}


tert-Butyl (S)-(1-(7-methoxy-2-methyl-4-oxo-4H-chromen-3-yl)-3-phenylprop-2yn-1-yl)carbamate (3f)<smiles>COc1ccc2c(=O)c([C@H](C#Cc3ccccc3)NC(=O)OCc3ccccc3)c(C)oc2c1</smiles>

Compound 3f: Prepared in $0.2 \mathrm{mmol}$ scale using 1.5 equiv of $2 \mathbf{a}$ at $70{ }^{\circ} \mathrm{C}$ for $62 \mathrm{~h} .72 \%$ yield $(60.0 \mathrm{mg})$, colorless oil, 95\% ee. HPLC (chiral ID column), hexane $/ i-\mathrm{PrOH}=70 / 30$, flow rate $0.8 \mathrm{ml} / \mathrm{min}, \lambda=254$ $\mathrm{nm}, \operatorname{tr}($ minor $)=14.04 \mathrm{~min}, \operatorname{tr}$ (major) $=24.39 \mathrm{~min}$.

$[\alpha]^{25} \mathrm{D}=+11.7\left(\mathrm{c}=1.0\right.$ in $\left.\mathrm{CH}_{2} \mathrm{Cl}_{2}\right)$.

${ }^{1} \mathrm{H}$ NMR $\left(400 \mathrm{MHz}, \mathrm{CDCl}_{3}\right) \delta 8.09(\mathrm{~d}, J=9.0 \mathrm{~Hz}, 1 \mathrm{H}), 7.45-7.36(\mathrm{~m}, 2 \mathrm{H}), 7.25-$ $7.22(\mathrm{~m}, 3 \mathrm{H}), 6.94(\mathrm{dd}, J=9.0,2.0 \mathrm{~Hz}, 1 \mathrm{H}), 6.79(\mathrm{~d}, J=2.0 \mathrm{~Hz}, 1 \mathrm{H}), 6.59$ (d, $J=8.8$ $\mathrm{Hz}, 1 \mathrm{H}), 5.85(\mathrm{~d}, J=9.4 \mathrm{~Hz}, 1 \mathrm{H}), 3.88(\mathrm{~s}, 3 \mathrm{H}), 2.61(\mathrm{~s}, 3 \mathrm{H}), 1.43(\mathrm{~s}, 9 \mathrm{H}) .{ }^{13} \mathrm{C}\{1 \mathrm{H}\}$ NMR $\left(100 \mathrm{MHz}, \mathrm{CDCl}_{3}\right) \delta 176.5,164.2,162.8,157.7,155.2,132.0,128.3,128.2$, $127.2,122.8,119.2,117.5,114.5,100.0,87.0,82.0,80.0,55.9,41.0,28.5,18.5$. HRMS (FTMS-ESI), m/z calcd for $\mathrm{C}_{25} \mathrm{H}_{25} \mathrm{NaO}_{5}^{+}\left([\mathrm{M}+\mathrm{Na}]^{+}\right) 442.1625$, found 442.1625 .

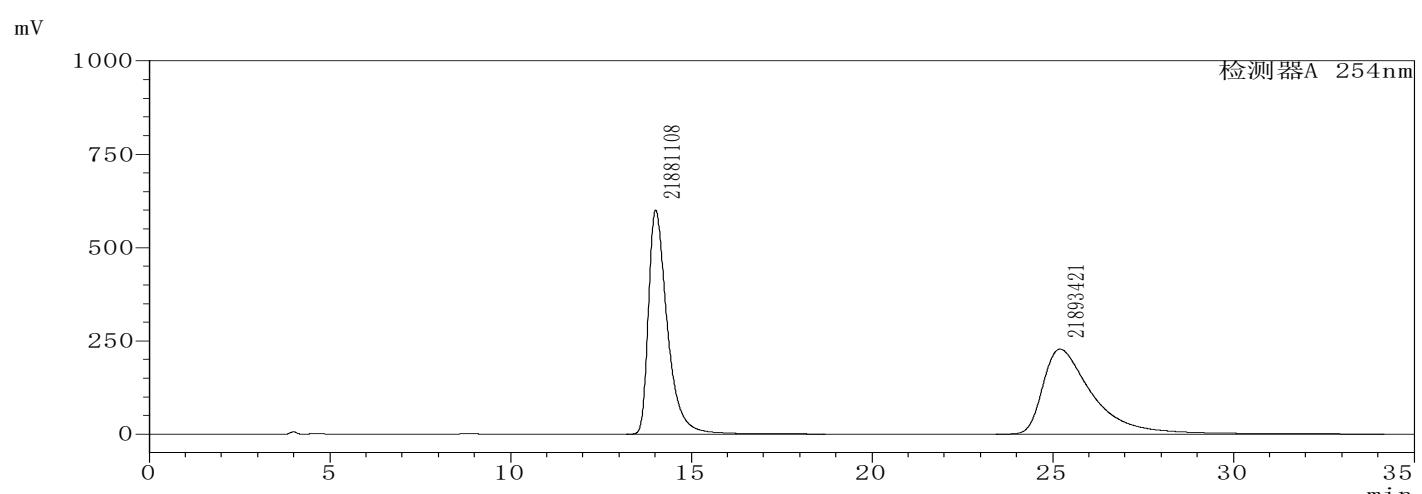

racemic

\begin{tabular}{|c|c|c|c|}
\hline & Retention Time & Area & \%Area \\
\hline 1 & 14.017 & 21881108 & 49.986 \\
\hline 2 & 25.204 & 21893421 & 50.014 \\
\hline
\end{tabular}

$\mathrm{mV}$

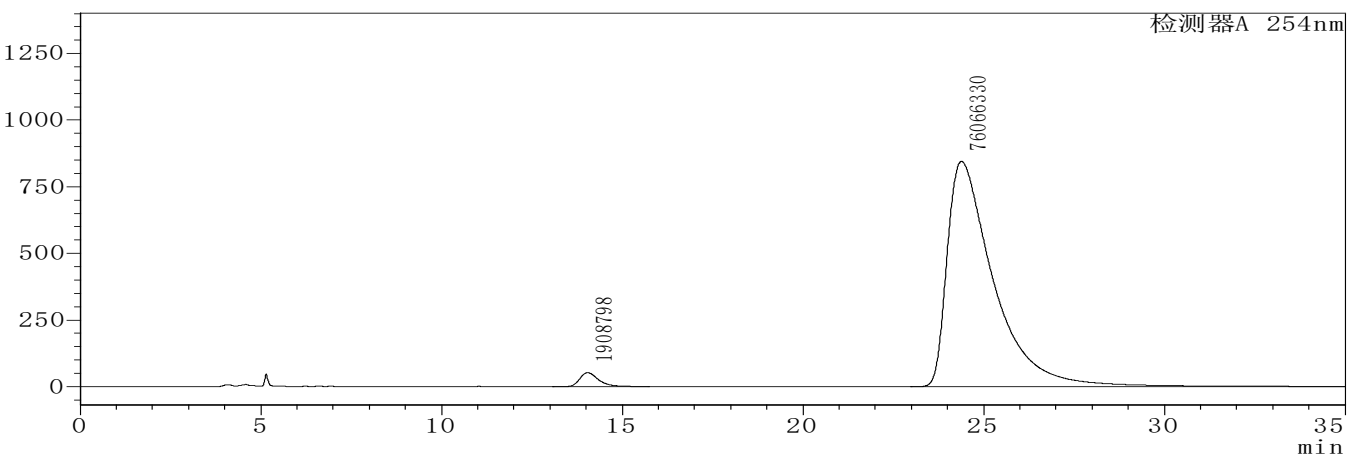

enantio-enriched

\begin{tabular}{|c|c|c|c|}
\hline & Retention Time & Area & \%Area \\
\hline 1 & 14.044 & 1908798 & 2.448 \\
\hline 2 & 24.390 & 76066330 & 97.552 \\
\hline
\end{tabular}


tert-Butyl (S)-(1-(7-(benzyloxy)-2-methyl-4-oxo-4H-chromen-3-yl)-3-phenylprop-2-yn-1-yl)carbamate $(3 g)$<smiles>Cc1oc2cc(OCc3ccccc3)ccc2c(=O)c1[C@H](C#Cc1ccccc1)NC(=O)O</smiles>

Compound 3g: Prepared in $0.2 \mathrm{mmol}$ scale using 1.5 equiv of $2 \mathbf{a}$ at $70{ }^{\circ} \mathrm{C}$ for 60 h. $88 \%$ yield $(87.0 \mathrm{mg})$, colorless oil, $96 \%$ ee. HPLC (chiral ID column), hexane $/ i-\mathrm{PrOH}=70 / 30$, flow rate $0.8 \mathrm{ml} / \mathrm{min}, \lambda=254$ $\mathrm{nm}, \operatorname{tr}($ minor $)=19.00 \mathrm{~min}, t \mathrm{r}$ (major $)=34.62 \mathrm{~min}$.

$[\alpha]^{25} \mathrm{D}=-1.7\left(\mathrm{c}=1.0\right.$ in $\left.\mathrm{CH}_{2} \mathrm{Cl}_{2}\right)$.

${ }^{1} \mathrm{H}$ NMR $\left(400 \mathrm{MHz}, \mathrm{CDCl}_{3}\right) \delta 8.11(\mathrm{~d}, J=9.0 \mathrm{~Hz}, 1 \mathrm{H}), 7.46-7.33(\mathrm{~m}, 7 \mathrm{H}), 7.28-$ $7.20(\mathrm{~m}, 3 \mathrm{H}), 7.03(\mathrm{dd}, J=9.0,2.0 \mathrm{~Hz}, 1 \mathrm{H}), 6.87(\mathrm{~d}, J=1.8 \mathrm{~Hz}, 1 \mathrm{H}), 6.60(\mathrm{~d}, J=9.0$ $\mathrm{Hz}, 1 \mathrm{H}), 5.86(\mathrm{~d}, J=9.4 \mathrm{~Hz}, 1 \mathrm{H}), 5.14(\mathrm{~s}, 2 \mathrm{H}), 2.61(\mathrm{~s}, 3 \mathrm{H}), 1.44(\mathrm{~s}, 9 \mathrm{H}) .{ }^{13} \mathrm{C}\{1 \mathrm{H}\}$ NMR $\left(100 \mathrm{MHz}, \mathrm{CDCl}_{3}\right) \delta 176.4,163.1,162.8,157.6,155.2,135.7,132.0,128.8$, 128.5, 128.3, 128.2, 127.6, 127.2, 122.8, 119.2, 117.7, 115.0, 101.1, 87.0, 82.0, 79.9, 70.6, 41.0, 28.5, 18.4. HRMS (FTMS-ESI), m/z calcd for $\mathrm{C}_{31} \mathrm{H}_{30} \mathrm{NO}_{5}{ }^{+}\left([\mathrm{M}+\mathrm{H}]^{+}\right)$ 496.2118, found 496.2109 .

$\mathrm{mV}$

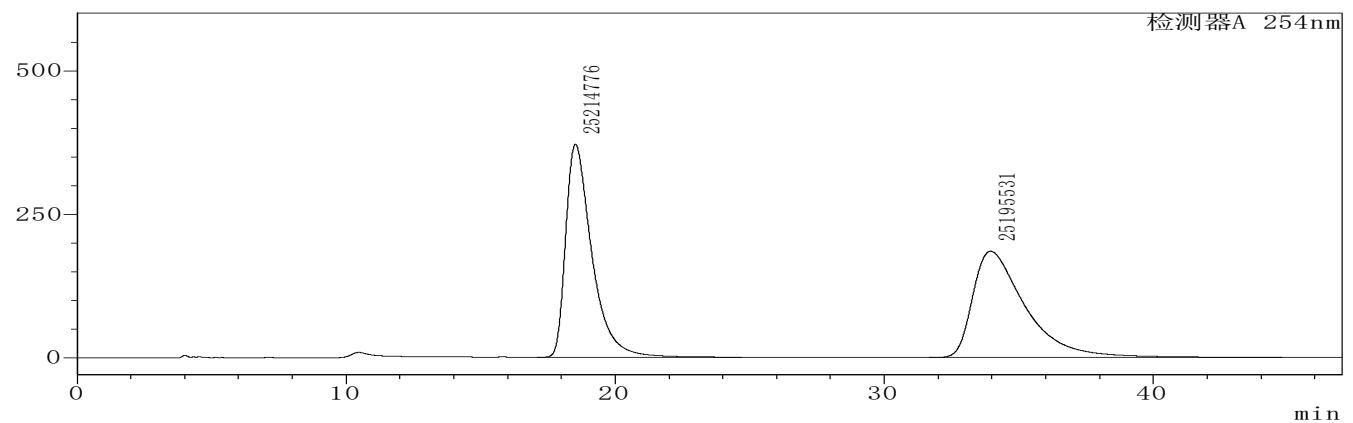

racemic

\begin{tabular}{|c|c|c|c|}
\hline & Retention Time & Area & \%Area \\
\hline 1 & 18.521 & 25214776 & 50.019 \\
\hline 2 & 33.950 & 25195531 & 49.981 \\
\hline
\end{tabular}

$\mathrm{mV}$

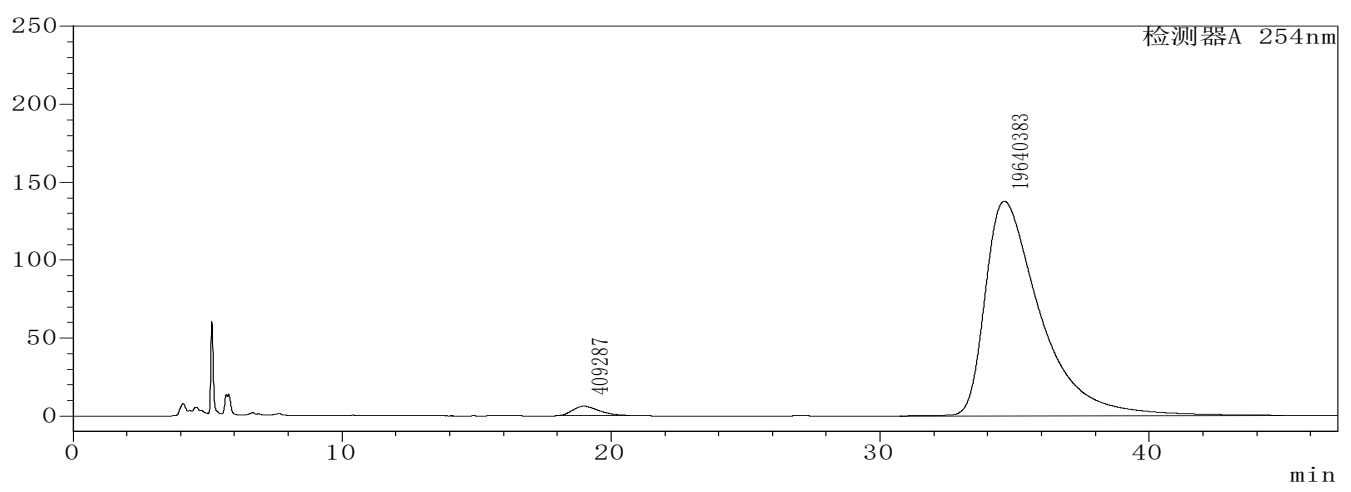

enantio-enriched

\begin{tabular}{|c|c|c|c|}
\hline & Retention Time & Area & \%Area \\
\hline 1 & 18.989 & 409287 & 2.041 \\
\hline 2 & 34.617 & 19640383 & 97.959 \\
\hline
\end{tabular}


tert-Butyl (S)-(1-(5-(benzyloxy)-2-methyl-4-oxo-4H-chromen-3-yl)-3-phenylprop-2-yn-1-yl)carbamate (3h)

(c)

Compound 3h: Prepared in $0.2 \mathrm{mmol}$ scale using 1.5 equiv of $1 \mathbf{a}$ at $70{ }^{\circ} \mathrm{C}$ for 78 h. $40 \%$ yield (40.0 mg), colorless oil, $97 \%$ ee. HPLC (chiral ID column), hexane $/ i-\mathrm{PrOH}=70 / 30$, flow rate $0.8 \mathrm{ml} / \mathrm{min}, \lambda=254 \mathrm{~nm}, t \mathrm{r}($ minor $)=10.26 \mathrm{~min}, t \mathrm{r}$

$($ major $)=15.36 \mathrm{~min}$.

$[\alpha]^{25} \mathrm{D}=+121.2\left(\mathrm{c}=1.0\right.$ in $\left.\mathrm{CH}_{2} \mathrm{Cl}_{2}\right)$.

${ }^{1} \mathrm{H}$ NMR $\left(400 \mathrm{MHz}, \mathrm{CDCl}_{3}\right) \delta 7.58(\mathrm{~d}, J=7.4 \mathrm{~Hz}, 2 \mathrm{H}), 7.48-7.35(\mathrm{~m}, 5 \mathrm{H}), 7.33-$ $7.22(\mathrm{~m}, 4 \mathrm{H}), 6.96(\mathrm{~d}, J=8.4 \mathrm{~Hz}, 1 \mathrm{H}), 6.79(\mathrm{~d}, J=8.2 \mathrm{~Hz}, 1 \mathrm{H}), 6.65(\mathrm{~d}, J=9.2 \mathrm{~Hz}$, $1 \mathrm{H}), 5.84(\mathrm{~d}, J=9.4 \mathrm{~Hz}, 1 \mathrm{H}), 5.32(\mathrm{~s}, 2 \mathrm{H}), 2.59(\mathrm{~s}, 3 \mathrm{H}), 1.44(\mathrm{~s}, 9 \mathrm{H}) .{ }^{13} \mathrm{C}\{1 \mathrm{H}\} \mathrm{NMR}$ $\left(150 \mathrm{MHz}, \mathrm{CDCl}_{3}\right) \delta 176.6,161.2,158.7,158.0,155.3,136.7,133.6,132.1,128.8$, 128.2, 128.2, 127.8, 126.8, 123.1, 120.5, 114.9, 110.3, 108.5, 87.4, 81.7, 79.8, 71.1, 41.1, 28.5, 18.2. HRMS (FTMS-ESI), $\mathrm{m} / \mathrm{z}$ calcd for $\mathrm{C}_{31} \mathrm{H}_{29} \mathrm{NaO}_{5}{ }^{+}\left([\mathrm{M}+\mathrm{Na}]^{+}\right)$ 518.1938 , found 518.1943 .

$\mathrm{mV}$

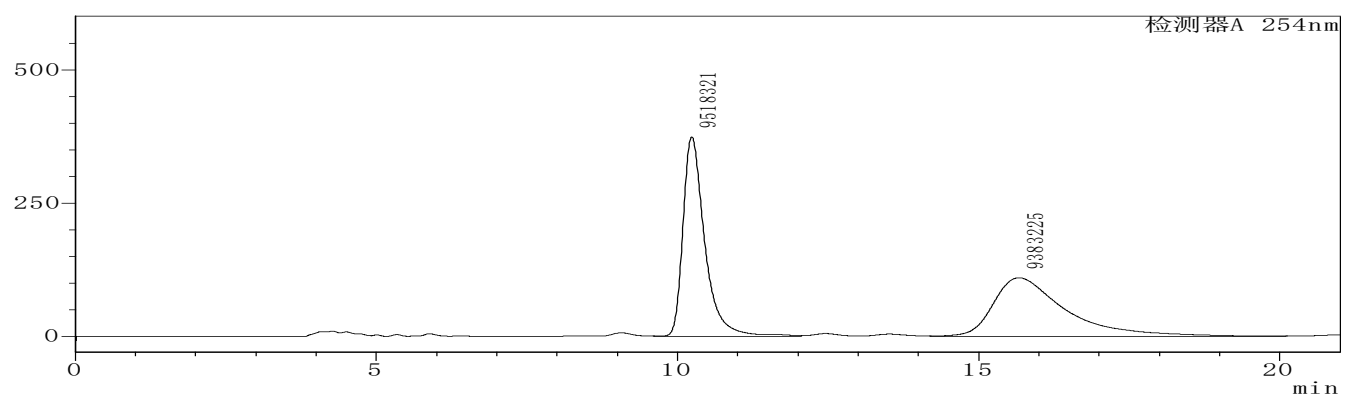

racemic

\begin{tabular}{|c|c|c|c|}
\hline & Retention Time & Area & \%Area \\
\hline 1 & 10.237 & 9518321 & 50.357 \\
\hline 2 & 15.668 & 9383225 & 49.643 \\
\hline
\end{tabular}

$\mathrm{mV}$

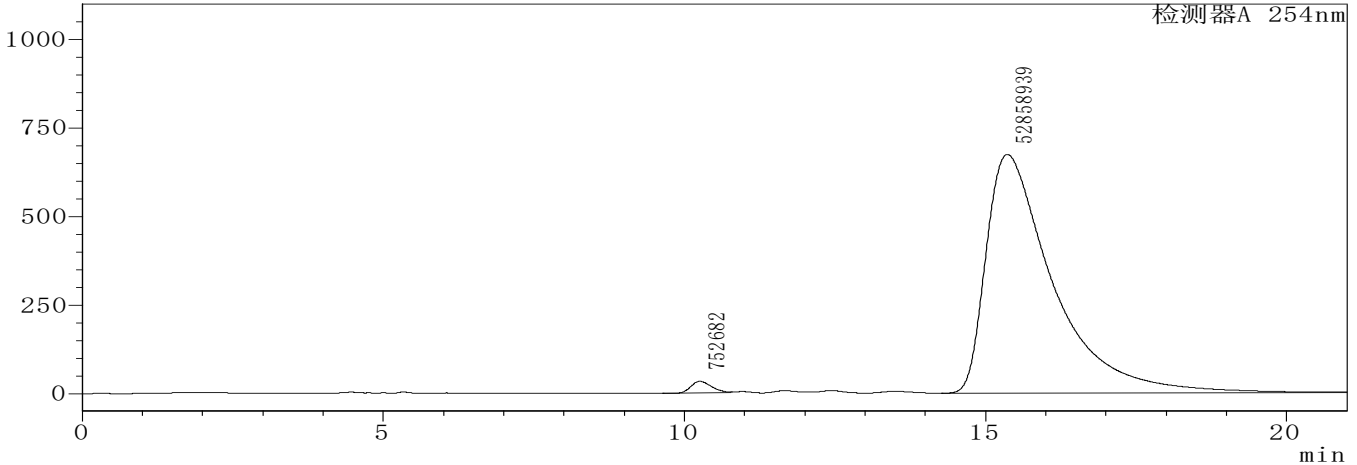

enantio-enriched

\begin{tabular}{|c|c|c|c|}
\hline & Retention Time & Area & \%Area \\
\hline 1 & 10.258 & 752682 & 1.404 \\
\hline 2 & 15.364 & 52858939 & 98.596 \\
\hline
\end{tabular}


tert-Butyl (S)-(1-(4-oxo-4H-chromen-3-yl)-3-phenylprop-2-yn-1-yl)carbamate (3i)<smiles>CC(C)(C)OC(=O)[C@H](C#Cc1ccccc1)c1coc2ccccc2c1=O</smiles>

Compound 3i: Prepared in $0.1 \mathrm{mmol}$ scale using 1.5 equiv of 2a at $90{ }^{\circ} \mathrm{C}$ for $60 \mathrm{~h} .59 \%$ yield $(22.0 \mathrm{mg})$, yellow solid; mp: 128.2-129.5 ${ }^{\circ} \mathrm{C}, 90 \%$ ee. HPLC (chiral IB column), hexane $/ i-\mathrm{PrOH}=70 / 30$, flow rate $0.8 \mathrm{ml} / \mathrm{min}, \lambda=254 \mathrm{~nm}, t \mathrm{r}$ $($ minor $)=6.85 \mathrm{~min}, t \mathrm{r}($ major $)=9.21 \mathrm{~min}$.

$[\alpha]^{25} \mathrm{D}=+44.5\left(\mathrm{c}=1.0\right.$ in $\left.\mathrm{CH}_{2} \mathrm{Cl}_{2}\right)$.

${ }^{1} \mathrm{H}$ NMR $\left(400 \mathrm{MHz}, \mathrm{CDCl}_{3}\right) \delta 8.26(\mathrm{~d}, J=8.0 \mathrm{~Hz}, 1 \mathrm{H}), 8.14(\mathrm{~s}, 1 \mathrm{H}), 7.69(\mathrm{t}, J=7.8$ $\mathrm{Hz}, 1 \mathrm{H}), 7.48(\mathrm{~d}, J=8.6 \mathrm{~Hz}, 1 \mathrm{H}), 7.45-7.39(\mathrm{~m}, 3 \mathrm{H}), 7.31-7.21(\mathrm{~m}, 4 \mathrm{H}), 5.98(\mathrm{~s}$, $1 \mathrm{H}), 5.64(\mathrm{~d}, J=9.0 \mathrm{~Hz}, 1 \mathrm{H}), 1.44(\mathrm{~s}, 9 \mathrm{H}) .{ }^{13} \mathrm{C}\{1 \mathrm{H}\} \mathrm{NMR}\left(150 \mathrm{MHz}, \mathrm{CDCl}_{3}\right) \delta 176.6$, $156.5,154.8,153.8,134.1,132.0,128.5,128.3,126.0,125.5,124.5,122.6,122.4$, 118.4, 86.4, 83.4, 80.3, 41.8, 28.5. HRMS (FTMS-ESI), m/z calcd for $\mathrm{C}_{23} \mathrm{H}_{21} \mathrm{NNaO}_{4}{ }^{+}$ $\left([\mathrm{M}+\mathrm{Na}]^{+}\right)$398.1363, found 398.1367. $\mathrm{mV}$

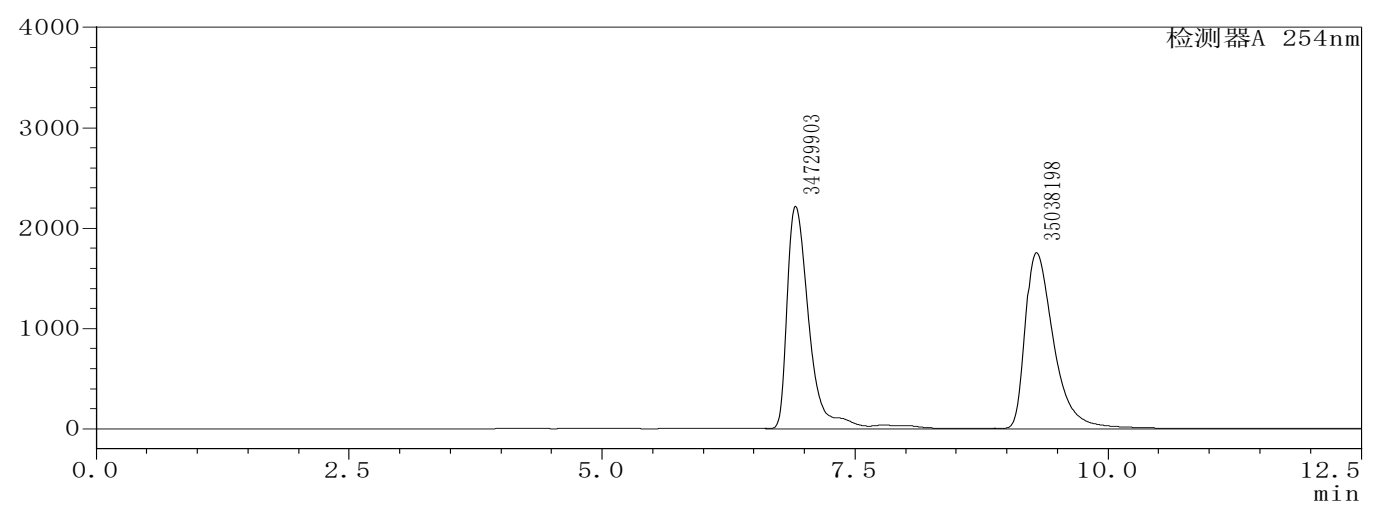

racemic

\begin{tabular}{|c|c|c|c|}
\hline & Retention Time & Area & \%Area \\
\hline 1 & 6.911 & 34729903 & 49.779 \\
\hline 2 & 9.289 & 35038198 & 50.221 \\
\hline
\end{tabular}

$\mathrm{mV}$

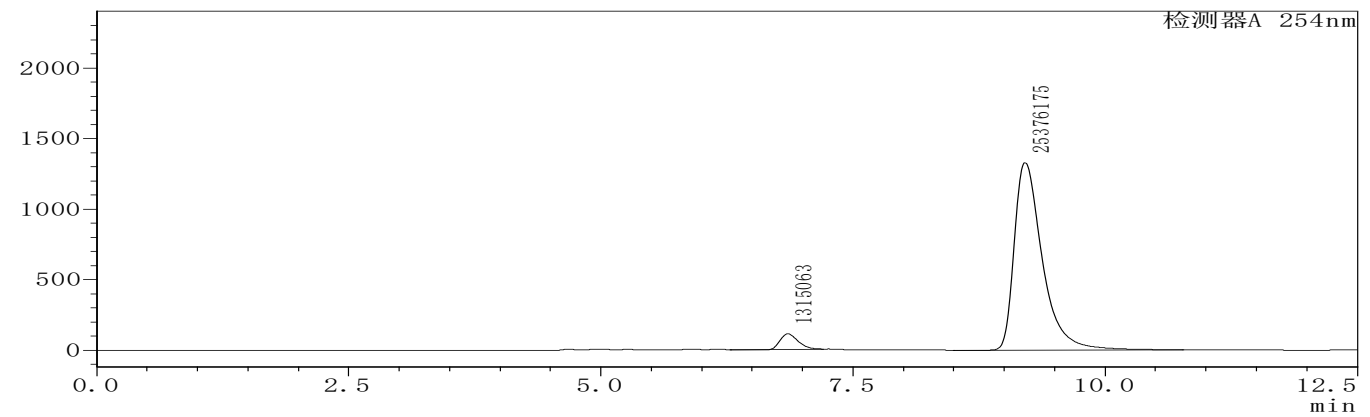

enantio-enriched

\begin{tabular}{|c|c|c|c|}
\hline & Retention Time & Area & \%Area \\
\hline 1 & 6.854 & 1315063 & 4.927 \\
\hline 2 & 9.207 & 25376175 & 95.073 \\
\hline
\end{tabular}


tert-Butyl (S)-(1-(2-ethyl-4-oxo-4H-chromen-3-yl)-3-phenylprop-2-yn-1-yl)carbamate (3j)<smiles>CCc1oc2ccccc2c(=O)c1[C@H](C#Cc1ccccc1)NC(C)(C)C</smiles>

Compound 3j: Prepared in $0.2 \mathrm{mmol}$ scale using 1.5 equiv of 1a at $70{ }^{\circ} \mathrm{C}$ for 72 h. $93 \%$ yield $(75.0 \mathrm{mg})$, colorless oil, $93 \%$ $e e$. HPLC (chiral ID column), hexane $/ i-\mathrm{PrOH}=70 / 30$, flow rate $0.8 \mathrm{ml} / \mathrm{min}, \lambda=254 \mathrm{~nm}, t \mathrm{r}$ (minor) $=8.23 \mathrm{~min}, t \mathrm{r}$ (major)

$=12.70 \mathrm{~min}$.

$[\alpha]^{25} \mathrm{D}=+35.5\left(\mathrm{c}=1.0\right.$ in $\left.\mathrm{CH}_{2} \mathrm{Cl}_{2}\right)$.

${ }^{1} \mathrm{H}$ NMR $\left(400 \mathrm{MHz}, \mathrm{CDCl}_{3}\right) \delta 8.21(\mathrm{~d}, J=8.0 \mathrm{~Hz}, 1 \mathrm{H}), 7.65(\mathrm{t}, J=7.8 \mathrm{~Hz}, 1 \mathrm{H}), 7.49$ $-7.35(\mathrm{~m}, 4 \mathrm{H}), 7.25(\mathrm{t}, J=5.0 \mathrm{~Hz}, 2 \mathrm{H}), 6.58(\mathrm{~d}, J=9.0 \mathrm{~Hz}, 1 \mathrm{H}), 5.93(\mathrm{~d}, J=9.4 \mathrm{~Hz}$, $1 \mathrm{H}), 3.03(\mathrm{td}, J=14.8,7.4 \mathrm{~Hz}, 1 \mathrm{H}), 2.91(\mathrm{td}, J=14.8,7.4 \mathrm{~Hz}, 1 \mathrm{H}), 1.50-1.35(\mathrm{~m}$, $12 \mathrm{H}) .{ }^{13} \mathrm{C}\{1 \mathrm{H}\} \mathrm{NMR}\left(150 \mathrm{MHz}, \mathrm{CDCl}_{3}\right) \delta 177.4,167.7,156.1,155.1,133.7,132.0$, 128.3, 128.2, 125.8, 125.1, 123.6, 122.9, 118.9, 117.9, 87.3, 82.0, 80.0, 40.7, 28.5, 25.8, 12.2. HRMS (FTMS-ESI), m/z calcd for $\mathrm{C}_{25} \mathrm{H}_{25} \mathrm{NNaO}_{4}{ }^{+}\left([\mathrm{M}+\mathrm{Na}]^{+}\right) 426.1676$, found 426.1679 .

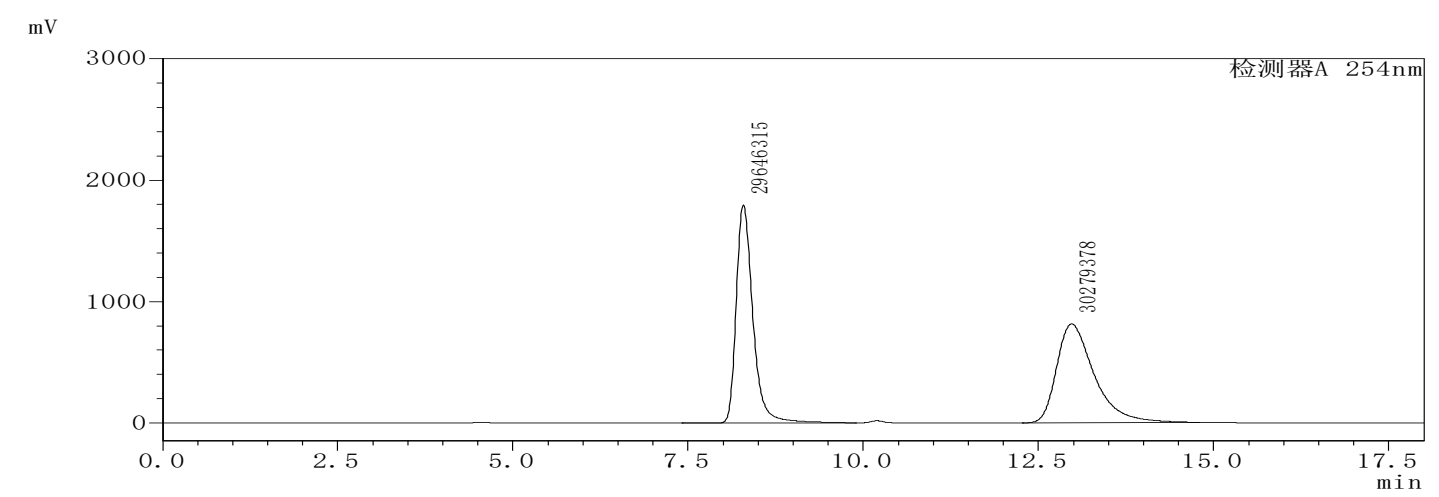

racemic

\begin{tabular}{|c|c|c|c|}
\hline & Retention Time & Area & \%Area \\
\hline 1 & 8.288 & 29646315 & 49.472 \\
\hline 2 & 12.973 & 30279378 & 50.528 \\
\hline
\end{tabular}

$\mathrm{mV}$

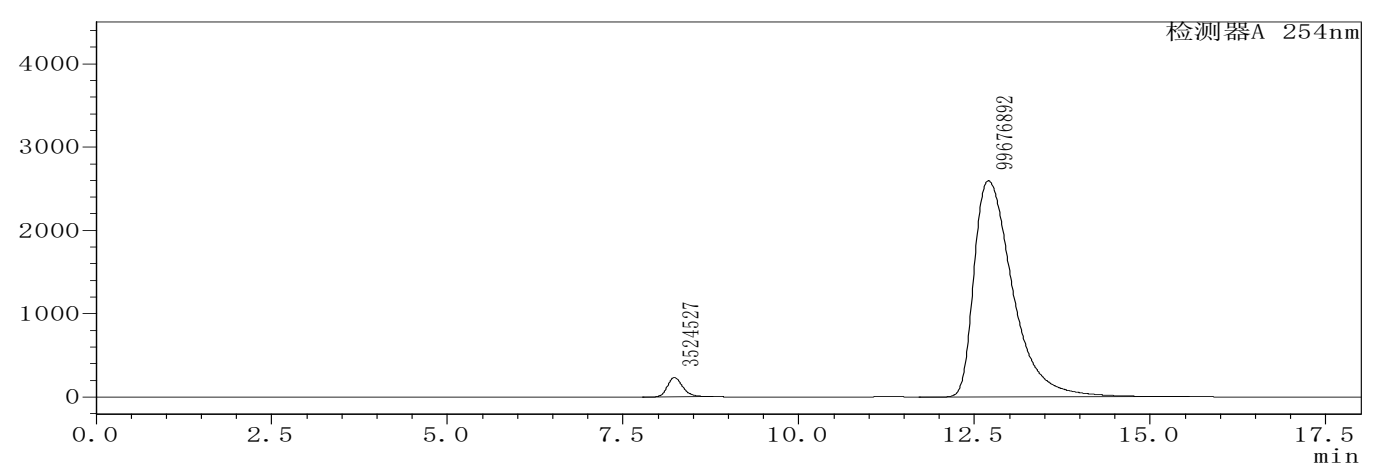

enantio-enriched

\begin{tabular}{|c|c|c|c|}
\hline & Retention Time & Area & \%Area \\
\hline 1 & 8.231 & 3524527 & 3.415 \\
\hline 2 & 12.701 & 99676892 & 96.585 \\
\hline
\end{tabular}


tert-Butyl (S)-(1-(4-oxo-2-propyl-4H-chromen-3-yl)-3-phenylprop-2-yn-1-yl)carbamate (3k)<smiles>CCCc1oc2ccccc2c(=O)c1[C@H](C#Cc1ccccc1)C(=O)OC(C)(C)C</smiles>

Compound 3k: Prepared in $0.2 \mathrm{mmol}$ scale using 1.5 equiv of $2 \mathbf{a}$ at $70{ }^{\circ} \mathrm{C}$ for $60 \mathrm{~h} .80 \%$ yield $(66.7 \mathrm{mg})$, colorless oil, $95 \%$ ee. HPLC (chiral ID column), hexane $/ i-\mathrm{PrOH}=80 / 20$, flow rate $0.8 \mathrm{ml} / \mathrm{min}, \lambda=254 \mathrm{~nm}, t \mathrm{r}$ (minor) $=9.02 \mathrm{~min}$, $t \mathrm{r}$

$($ major $)=14.65 \mathrm{~min}$.

$[\alpha]^{25}=+29.3\left(\mathrm{c}=1.0\right.$ in $\left.\mathrm{CH}_{2} \mathrm{Cl}_{2}\right)$.

${ }^{1} \mathrm{H}$ NMR $\left(400 \mathrm{MHz}, \mathrm{CDCl}_{3}\right) \delta 8.21(\mathrm{dd}, J=8.0,1.4 \mathrm{~Hz}, 1 \mathrm{H}), 7.65$ (ddd, $J=8.8,7.2$, $1.8 \mathrm{~Hz}, 1 \mathrm{H}), 7.44-7.35$ (m, 4H), $7.27-7.22$ (m, 3H), 6.59 (d, J=9.4 Hz, 1H), 5.92 $(\mathrm{d}, J=9.4 \mathrm{~Hz}, 1 \mathrm{H}), 3.08-2.95(\mathrm{~m}, 1 \mathrm{H}), 2.88-2.75(\mathrm{~m}, 1 \mathrm{H}), 1.89(\mathrm{dq}, J=14.8,7.4$ $\mathrm{Hz}, 2 \mathrm{H}), 1.43(\mathrm{~s}, 9 \mathrm{H}), 1.09(\mathrm{t}, J=7.4 \mathrm{~Hz}, 3 \mathrm{H}) .{ }^{13} \mathrm{C}\{1 \mathrm{H}\} \mathrm{NMR}\left(150 \mathrm{MHz}, \mathrm{CDCl}_{3}\right) \delta$ $177.4,166.7,156.0,155.1,133.7,132.0,128.3,128.2$, 125.8, 125.1, 123.6, 122.9, 119.5, 117. 9, 87.3, 81.9, 80.0, 40.8, 34.1, 28.5, 21.3, 13.9. HRMS (TOFMS-ESI), $\mathrm{m} / \mathrm{z}$ calcd for $\mathrm{C}_{26} \mathrm{H}_{28} \mathrm{NO}_{4}{ }^{+}\left([\mathrm{M}+\mathrm{H}]^{+}\right)$418.2013, found 418.2019.

$\mathrm{mV}$

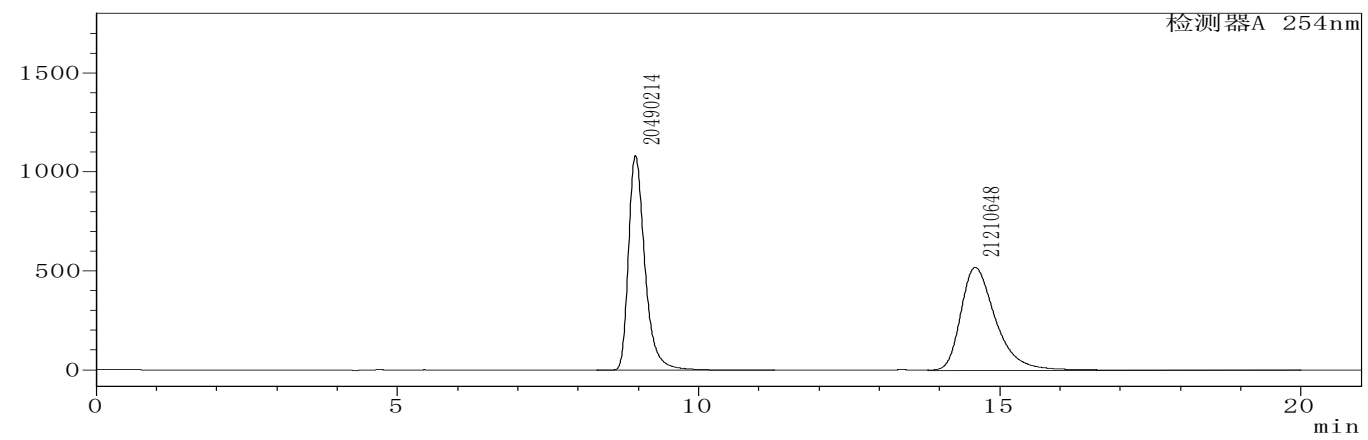

racemic

\begin{tabular}{|c|c|c|c|}
\hline & Retention Time & Area & \%Area \\
\hline 1 & 8.953 & 20490214 & 49.136 \\
\hline 2 & 14.595 & 21210648 & 50.864 \\
\hline
\end{tabular}

$\mathrm{mV}$

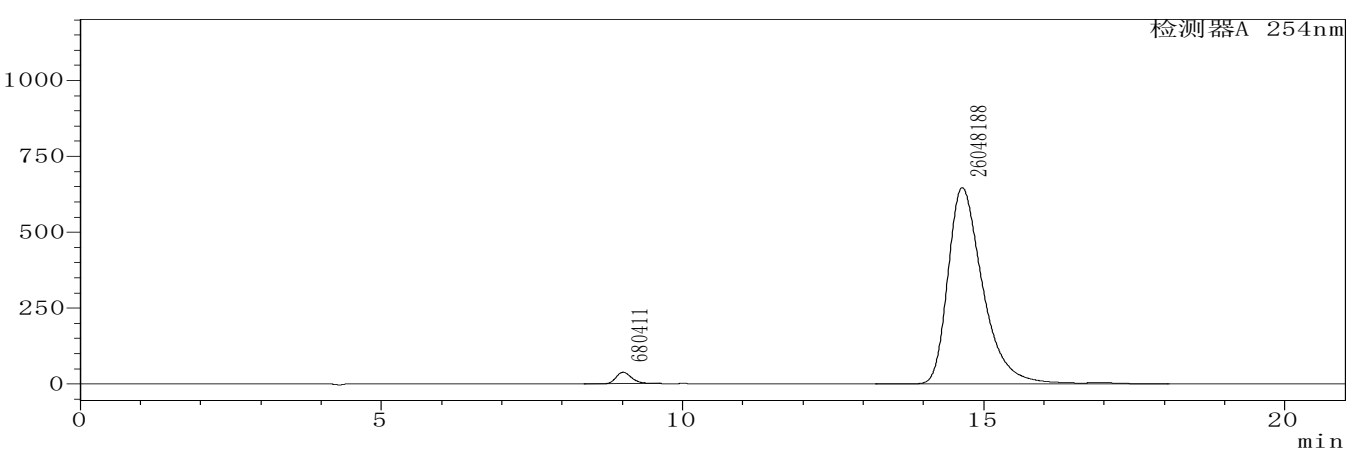

enantio-enriched

\begin{tabular}{|c|c|c|c|}
\hline & Retention Time & Area & \%Area \\
\hline 1 & 9.015 & 680411 & 2.546 \\
\hline 2 & 14.646 & 26048188 & 97.454 \\
\hline
\end{tabular}


tert-Butyl (S)-(1-(4-oxo-2-pentyl-4H-chromen-3-yl)-3-phenylprop-2-yn-1-yl)carbamate (3I)

$\begin{aligned} & \text { Compound 3l: Prepared in } 0.2 \mathrm{mmol} \text { scale using } 1.5 \text { equiv of } \\ & \mathbf{2 a} \text { at } 70^{\circ} \mathrm{C} \text { for } 60 \mathrm{~h} .95 \% \text { yield }(85.0 \mathrm{mg}), \text { colorless oil, } 95 \%\end{aligned}$
$\begin{aligned} & \text { ee. } \mathrm{HPLC} \text { (chiral ID column), hexane } / \mathrm{i}-\mathrm{PrOH}=70 / 30, \text { flow } \\ & \text { rate } 0.8 \mathrm{ml} / \mathrm{min}, \lambda=254 \mathrm{~nm}, t \mathrm{r} \text { (minor) }=6.70 \mathrm{~min}, \mathrm{tr} \text { (major) } \\ & =9.08 \mathrm{~min} .\end{aligned}$ $[\alpha]^{25}{ }_{\mathrm{D}}=+14.0\left(\mathrm{c}=1.0\right.$ in $\left.\mathrm{CH}_{2} \mathrm{Cl}_{2}\right)$.

${ }^{1} \mathrm{H}$ NMR $\left(400 \mathrm{MHz}, \mathrm{CDCl}_{3}\right) \delta 8.21(\mathrm{~d}, J=7.8 \mathrm{~Hz}, 1 \mathrm{H}), 7.68-7.61(\mathrm{~m}, 1 \mathrm{H}), 7.46-$ $7.30(\mathrm{~m}, 4 \mathrm{H}), 7.28-7.17(\mathrm{~m}, 3 \mathrm{H}), 6.59(\mathrm{~d}, J=9.4 \mathrm{~Hz}, 1 \mathrm{H}), 5.92(\mathrm{~d}, J=9.4 \mathrm{~Hz}, 1 \mathrm{H})$, $3.09-2.97(\mathrm{~m}, 1 \mathrm{H}), 2.89-2.76(\mathrm{~m}, 1 \mathrm{H}), 1.93-1.78(\mathrm{~m}, 2 \mathrm{H}), 1.53-1.34(\mathrm{~m}, 13 \mathrm{H})$, $0.90(\mathrm{t}, J=7.0 \mathrm{~Hz}, 3 \mathrm{H}) .{ }^{13} \mathrm{C}\{1 \mathrm{H}\} \mathrm{NMR}\left(100 \mathrm{MHz}, \mathrm{CDCl}_{3}\right) \delta 177.4,167.0,156.0$, 155.1, 133.7, 131.9, 128.2, 128.2, 125.8, 125.1, 123.6, 122.9, 119.3, 117.9, 87.3, 81.9, 79.9, 40.8, 32.3, 31.5, 28.5, 27.6, 22.6, 14.0. HRMS (TOFMS-ESI), m/z calcd for $\mathrm{C}_{28} \mathrm{H}_{32} \mathrm{NO}_{4}{ }^{+}\left([\mathrm{M}+\mathrm{H}]^{+}\right) 446.2326$, found 446.2335.

$\mathrm{mV}$

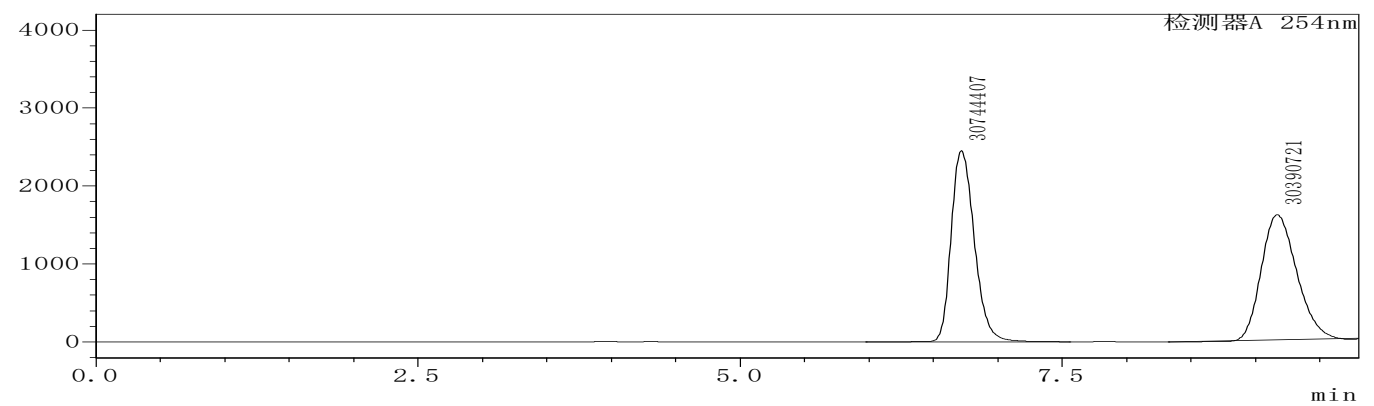

racemic

\begin{tabular}{|c|c|c|c|}
\hline & Retention Time & Area & \%Area \\
\hline 1 & 6.719 & 30744407 & 50.289 \\
\hline 2 & 9.170 & 30390721 & 49.711 \\
\hline
\end{tabular}

$\mathrm{mV}$

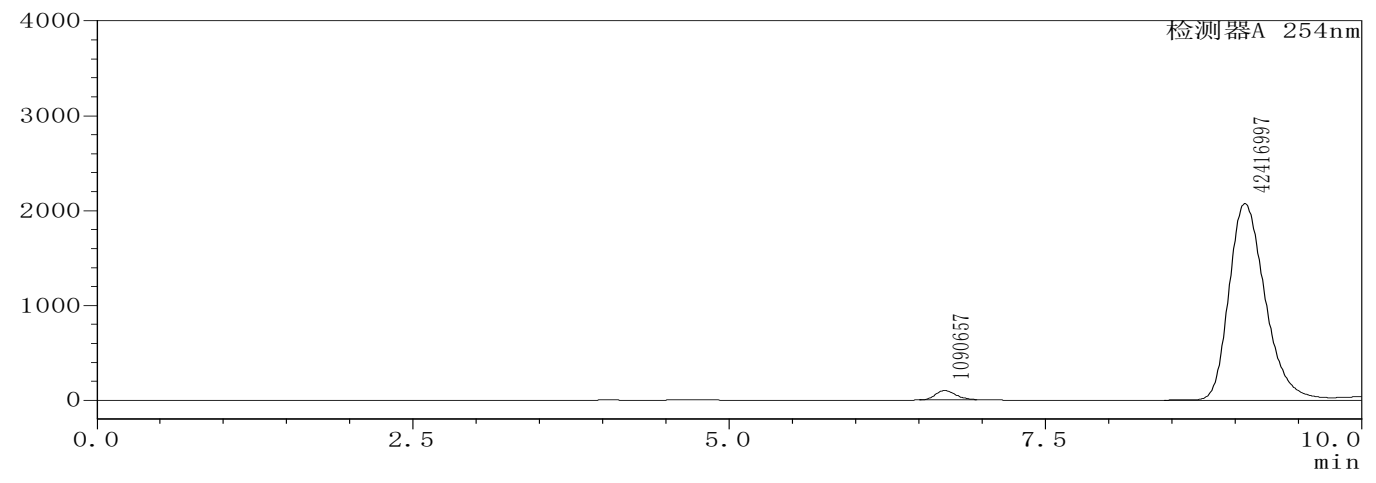

enantio-enriched

\begin{tabular}{|c|c|c|c|}
\hline & Retention Time & Area & \%Area \\
\hline 1 & 6.704 & 1090657 & 2.507 \\
\hline 2 & 9.079 & 42416997 & 97.493 \\
\hline
\end{tabular}


tert-Butyl (S)-(1-(2-(methoxymethyl)-4-oxo-4H-chromen-3-yl)-3-phenylprop-2yn-1-yl)carbamate (3m)<smiles>COCc1oc2ccccc2c(=O)c1C(C#Cc1ccccc1)NC(C)(C)C</smiles>

Compound 3m: Prepared in $0.2 \mathrm{mmol}$ scale using 1.5 equiv of $2 \mathbf{a}$ at $70{ }^{\circ} \mathrm{C}$ for $60 \mathrm{~h} .75 \%$ yield $(63.1 \mathrm{mg})$, colorless oil, $96 \%$ ee. HPLC (chiral ID column), hexane $/ i-\mathrm{PrOH}=70 / 30$, flow rate $0.8 \mathrm{ml} / \mathrm{min}, \lambda=254 \mathrm{~nm}, t \mathrm{r}$ (minor) $=11.25 \mathrm{~min}, t \mathrm{r}$ (major) $=17.21 \mathrm{~min}$.

$[\alpha]^{25} \mathrm{D}=+35.4\left(\mathrm{c}=1.0\right.$ in $\left.\mathrm{CH}_{2} \mathrm{Cl}_{2}\right)$.

${ }^{1} \mathrm{H} \mathrm{NMR}\left(400 \mathrm{MHz}, \mathrm{CDCl}_{3}\right) \delta 8.22(\mathrm{dd}, J=7.8,0.8 \mathrm{~Hz}, 1 \mathrm{H}), 7.68(\mathrm{t}, J=7.8 \mathrm{~Hz}, 1 \mathrm{H})$, $7.47(\mathrm{~d}, J=8.4 \mathrm{~Hz}, 1 \mathrm{H}), 7.43-7.35(\mathrm{~m}, 3 \mathrm{H}), 7.28-7.19(\mathrm{~m}, 3 \mathrm{H}), 6.42(\mathrm{~d}, J=9.2 \mathrm{~Hz}$, $1 \mathrm{H}), 6.10(\mathrm{~d}, J=9.2 \mathrm{~Hz}, 1 \mathrm{H}), 4.91(\mathrm{~d}, J=13.0 \mathrm{~Hz}, 1 \mathrm{H}), 4.53(\mathrm{~d}, J=13.0 \mathrm{~Hz}, 1 \mathrm{H})$, $3.53(\mathrm{~s}, 3 \mathrm{H}), 1.42(\mathrm{~s}, 9 \mathrm{H}) .{ }^{13} \mathrm{C}\{1 \mathrm{H}\} \mathrm{NMR}\left(100 \mathrm{MHz}, \mathrm{CDCl}_{3}\right) \delta 177.3,161.1,155.9$, 155.0, 134.2, 132.0, 128.3, 128.2, 125.9, 125.4, 123.7, 122.8, 121.3, 118.2, 86.9, 82.2, 80.0, 69.8, 59.2, 59.1, 39.9, 28.5. HRMS (TOFMS-ESI), $\mathrm{m} / \mathrm{z}$ calcd for $\mathrm{C}_{25} \mathrm{H}_{26} \mathrm{NO}_{5}{ }^{+}$ $\left([\mathrm{M}+\mathrm{H}]^{+}\right) 420.1805$, found 420.1815 .

$\mathrm{mV}$

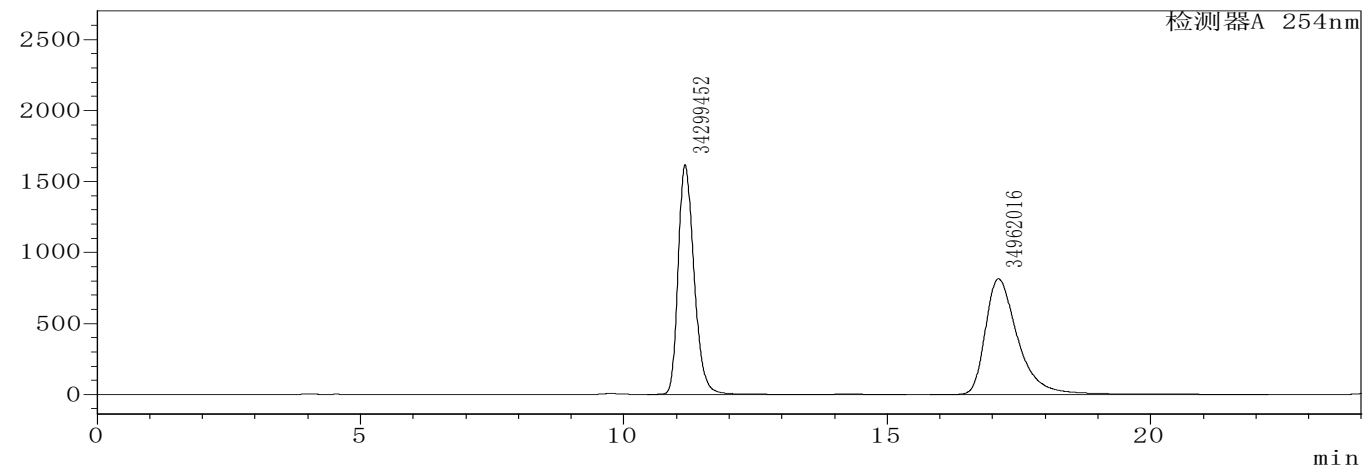

racemic

\begin{tabular}{|c|c|c|c|}
\hline & Retention Time & Area & \%Area \\
\hline 1 & 11.162 & 34299452 & 49.522 \\
\hline 2 & 17.111 & 34962016 & 50.478 \\
\hline
\end{tabular}

$\mathrm{mV}$

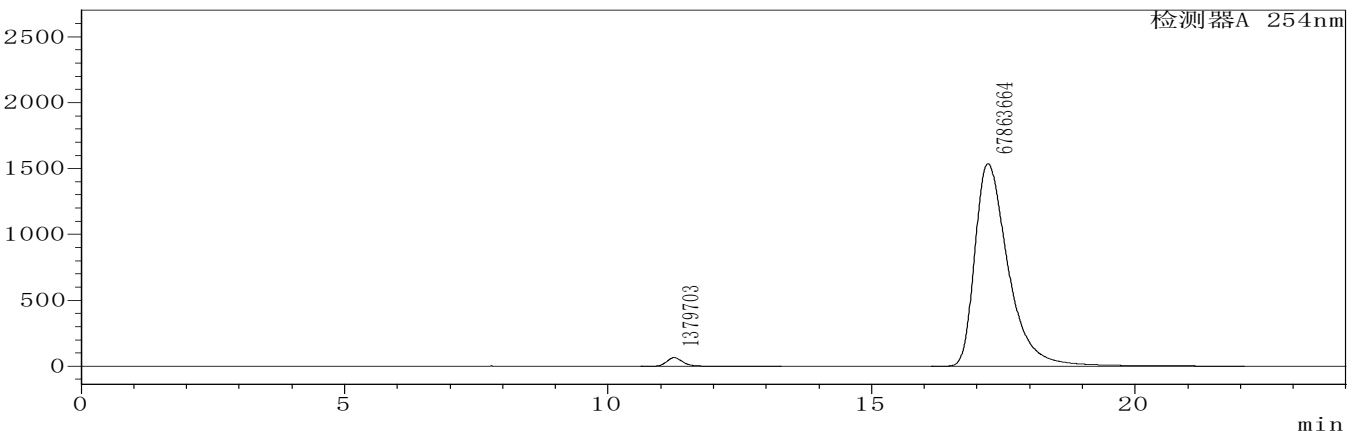

enantio-enriched

\begin{tabular}{|c|c|c|c|}
\hline & Retention Time & Area & \%Area \\
\hline 1 & 11.252 & 1379703 & 1.993 \\
\hline 2 & 17.210 & 67863664 & 98.007 \\
\hline
\end{tabular}


tert-Butyl (S)-(1-(4-oxo-2-phenethyl-4H-chromen-3-yl)-3-phenylprop-2-yn-1-yl) carbamate (3n)<smiles>CC(C)(C)OC(=O)N[C@H](C#Cc1ccccc1)c1c(CCc2ccccc2)oc2ccccc2c1=O</smiles>

Compound 3n: Prepared in $0.2 \mathrm{mmol}$ scale using 1.2 equiv of $1 \mathbf{a}$ at $70{ }^{\circ} \mathrm{C}$ for $72 \mathrm{~h} .78 \%$ yield $(75.0 \mathrm{mg})$, colorless oil, $95 \%$ ee. HPLC (chiral ID column), hexane $/ i$-PrOH $=70 / 30$, flow rate $0.8 \mathrm{ml} / \mathrm{min}, \lambda=254 \mathrm{~nm}, t \mathrm{r}$ (minor) $=9.24 \mathrm{~min}, t \mathrm{r}$

$($ major $)=13.51 \mathrm{~min}$.

$[\alpha]^{25} \mathrm{D}=+3.8\left(\mathrm{c}=1.0\right.$ in $\left.\mathrm{CH}_{2} \mathrm{Cl}_{2}\right)$.

${ }^{1} \mathrm{H}$ NMR $\left(400 \mathrm{MHz}, \mathrm{CDCl}_{3}\right) \delta 8.21(\mathrm{~d}, J=7.8 \mathrm{~Hz}, 1 \mathrm{H}), 7.65(\mathrm{t}, J=7.8 \mathrm{~Hz}, 1 \mathrm{H}), 7.43$ $-7.36(\mathrm{~m}, 4 \mathrm{H}), 7.32-7.22(\mathrm{~m}, 7 \mathrm{H}), 7.21-7.17(\mathrm{~m}, 1 \mathrm{H}), 6.53(\mathrm{~d}, J=9.0 \mathrm{~Hz}, 1 \mathrm{H})$, $5.98(\mathrm{~d}, J=9.4 \mathrm{~Hz}, 1 \mathrm{H}), 3.38-3.25(\mathrm{~m}, 1 \mathrm{H}), 3.22-3.10(\mathrm{~m}, 3 \mathrm{H}), 1.44(\mathrm{~s}, 9 \mathrm{H})$. ${ }^{13} \mathrm{C}\{1 \mathrm{H}\} \mathrm{NMR}\left(150 \mathrm{MHz}, \mathrm{CDCl}_{3}\right) \delta 177.4,165.6,156.0,155.1,139.9,133.8,132.0$, 128.8, 128.6, 128.3, 128.2, 126.7, 125.9, 125.2, 123.6, 122.9, 119.6, 117.9, 87.2, 82.1, 80.0, 40.7, 34.3, 33.9, 28.5. HRMS (FTMS-ESI), m/z calcd for $\mathrm{C}_{31} \mathrm{H}_{29} \mathrm{NNaO}_{4}{ }^{+}([\mathrm{M}+$ $\mathrm{Na}]^{+}$) 502.1989, found 502.1985.

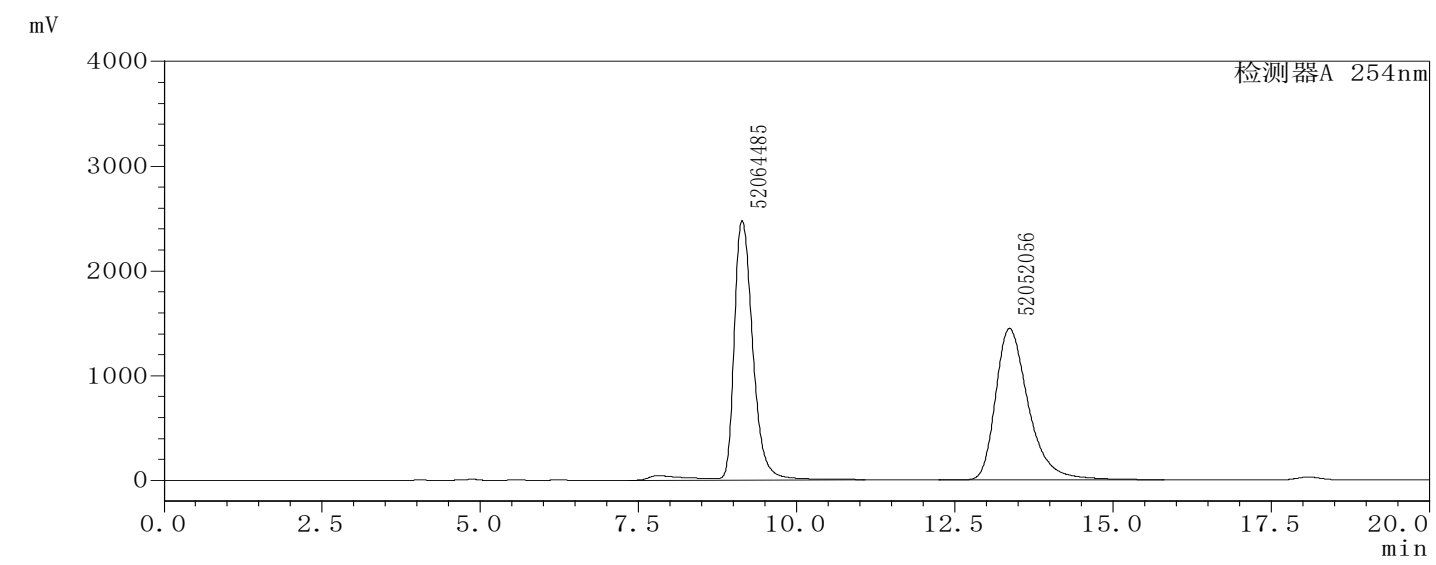

racemic

\begin{tabular}{|c|c|c|c|}
\hline & Retention Time & Area & \%Area \\
\hline 1 & 9.138 & 52064485 & 50.006 \\
\hline 2 & 13.366 & 52052056 & 49.994 \\
\hline
\end{tabular}

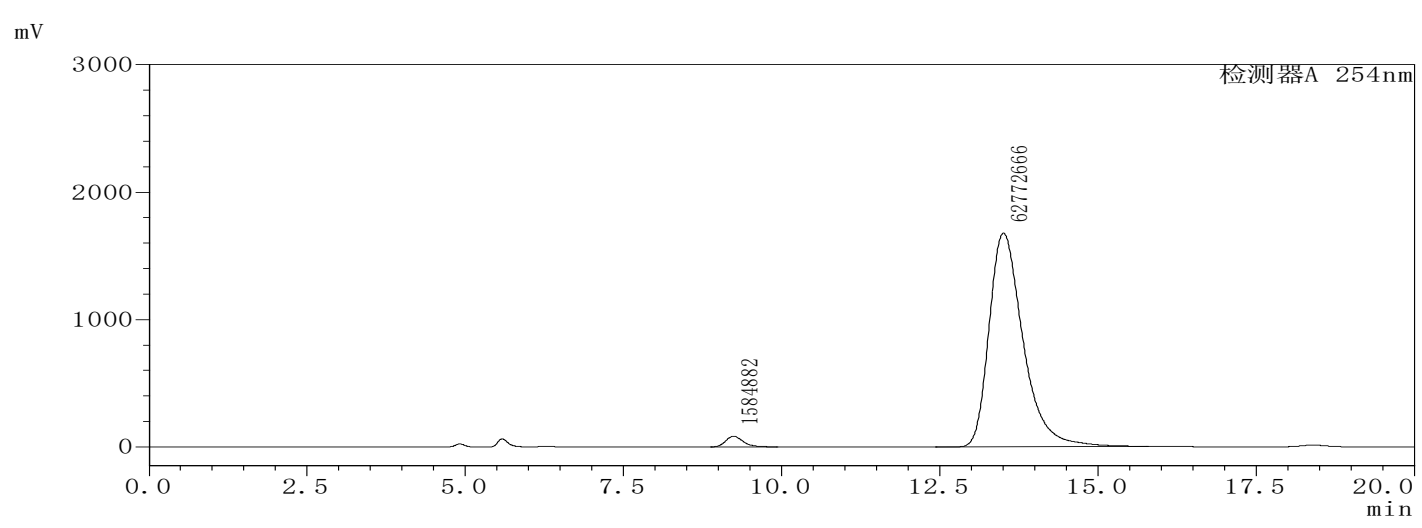

enantio-enriched

\begin{tabular}{|c|c|c|c|}
\hline & Retention Time & Area & \%Area \\
\hline 1 & 9.240 & 1584882 & 2.463 \\
\hline 2 & 13.507 & 62772666 & 97.537 \\
\hline
\end{tabular}


tert-Butyl (S)-(3-(2-bromophenyl)-1-(2-methyl-4-oxo-4H-chromen-3-yl)prop-2yn-1-yl)carbamate (3o)<smiles>Cc1oc2ccccc2c(=O)c1[C@H](C#Cc1ccccc1Br)NC(=O)OC(C)(C)C</smiles>

Compound 3o: Prepared in $0.2 \mathrm{mmol}$ scale using 1.5 equiv of $1 \mathrm{a}$ at $70{ }^{\circ} \mathrm{C}$ for $60 \mathrm{~h} .97 \%$ yield $(91.0 \mathrm{mg})$, colorless oil, 95\% ee. HPLC (chiral ID column), hexane $/ i-\mathrm{PrOH}=70 / 30$, flow rate $0.8 \mathrm{ml} / \mathrm{min}, \lambda=254$ $\mathrm{nm}, \operatorname{tr}($ minor $)=11.47 \mathrm{~min}, \mathrm{tr}($ major $)=17.83 \mathrm{~min}$.

$[\alpha]^{25} \mathrm{D}=+72.9\left(\mathrm{c}=1.0\right.$ in $\left.\mathrm{CH}_{2} \mathrm{Cl}_{2}\right)$.

${ }^{1} \mathrm{H}$ NMR $\left(400 \mathrm{MHz}, \mathrm{CDCl}_{3}\right) \delta 8.20(\mathrm{~d}, J=8.0 \mathrm{~Hz}, 1 \mathrm{H}), 7.67-7.60(\mathrm{~m}, 1 \mathrm{H}), 7.52-$ 7.35 (m, 4H), $7.26-7.23(\mathrm{~m}, 1 \mathrm{H}), 7.15$ (dt, $J=34.8,7.8 \mathrm{~Hz}, 1 \mathrm{H}), 6.56$ (dd, $J=17.4$, $9.4 \mathrm{~Hz}, 1 \mathrm{H}), 5.91(\mathrm{dd}, J=23.8,9.4 \mathrm{~Hz}, 1 \mathrm{H}), 2.66(\mathrm{~d}, J=5.6 \mathrm{~Hz}, 3 \mathrm{H}), 1.43(\mathrm{~s}, 9 \mathrm{H})$. ${ }^{13} \mathrm{C}\{1 \mathrm{H}\} \mathrm{NMR}\left(100 \mathrm{MHz}, \mathrm{CDCl}_{3}\right) \delta 177.1,163.4,155.9,155.2,133.8,132.3,132.0$, 129.5, 128.3, 128.2, 126.9, 125.8, 125.1, 123.6, 122.8, 117.9, 91.6, 86.8, 80.0, 41.0, 28.5, 18.6. HRMS (TOFMS-ESI), $\mathrm{m} / \mathrm{z}$ calcd for $\mathrm{C}_{24} \mathrm{H}_{23} \mathrm{BrNO}_{4}{ }^{+}\left([\mathrm{M}+\mathrm{H}]^{+}\right) 468.0805$ (470.0785 for ${ }^{81} \mathrm{Br}$ ), found 468.0810 (470.0794 for ${ }^{81} \mathrm{Br}$ ).

$\mathrm{mV}$

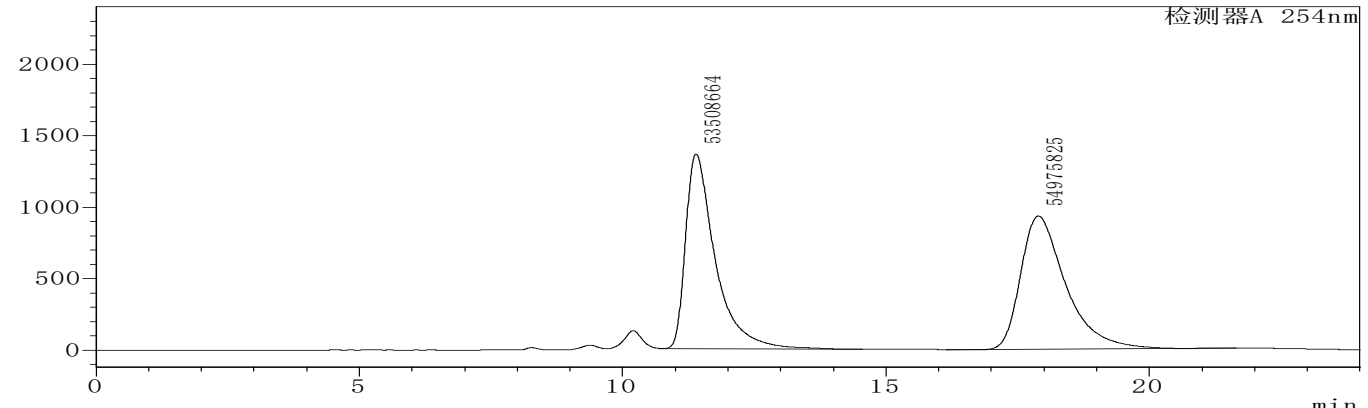

racemic

\begin{tabular}{|c|c|c|c|}
\hline & Retention Time & Area & \%Area \\
\hline 1 & 11.394 & 53508664 & 49.324 \\
\hline 2 & 17.891 & 54975825 & 50.676 \\
\hline
\end{tabular}

$\mathrm{mV}$

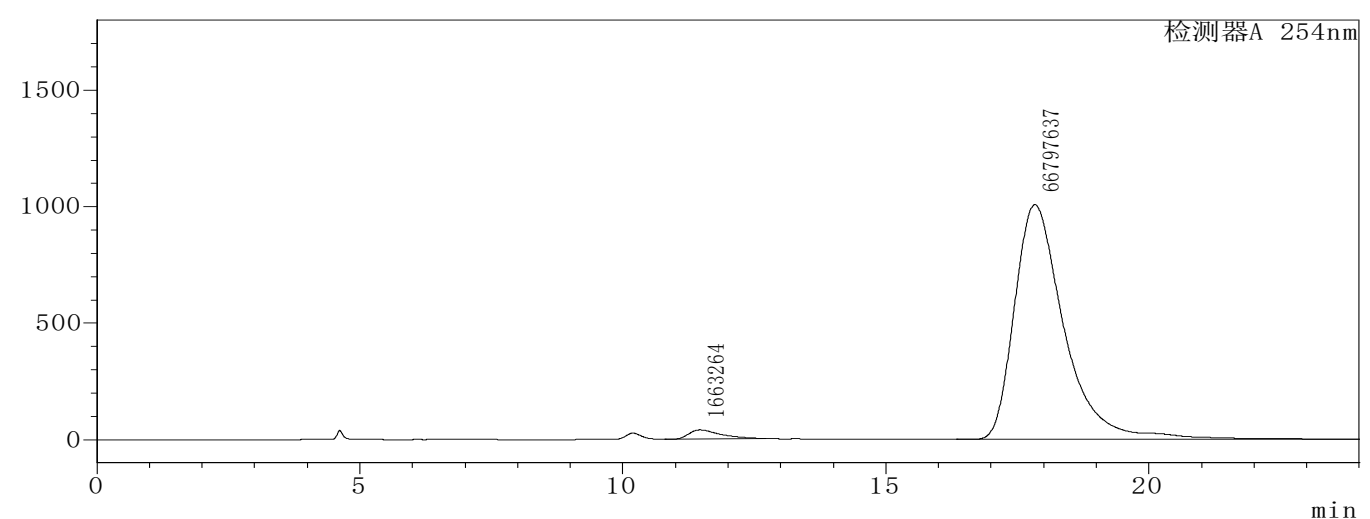

enantio-enriched

\begin{tabular}{|c|c|c|c|}
\hline & Retention Time & Area & \%Area \\
\hline 1 & 11.467 & 1663264 & 2.430 \\
\hline 2 & 17.832 & 66797637 & 97.570 \\
\hline
\end{tabular}


tert-Butyl (S)-(3-(2-methoxyphenyl)-1-(2-methyl-4-oxo-4H-chromen-3-yl)prop2-yn-1-yl)carbamate (3p)<smiles>COC(=O)N[C@@H](C#Cc1ccccc1OC)c1c(C)oc2ccccc2c1=O</smiles>

Compound 3p: Prepared in $0.2 \mathrm{mmol}$ scale using 1.5 equiv of $1 \mathrm{a}$ at $70{ }^{\circ} \mathrm{C}$ for $65 \mathrm{~h} .95 \%$ yield $(79.4 \mathrm{mg})$, colorless oil, 95\% ee. HPLC (chiral IC column), hexane $/ i-\mathrm{PrOH}=70 / 30$, flow rate $0.8 \mathrm{ml} / \mathrm{min}, \lambda=254$ $\mathrm{nm}, \operatorname{tr}($ minor $)=20.59 \mathrm{~min}, \operatorname{tr}($ major $)=26.90 \mathrm{~min}$.

$[\alpha]^{25} \mathrm{D}=+60.6\left(\mathrm{c}=1.0\right.$ in $\left.\mathrm{CH}_{2} \mathrm{Cl}_{2}\right)$.

${ }^{1} \mathrm{H}$ NMR $\left(400 \mathrm{MHz}, \mathrm{CDCl}_{3}\right) \delta 8.20(\mathrm{~d}, J=8.0 \mathrm{~Hz}, 1 \mathrm{H}), 7.63(\mathrm{t}, J=7.8 \mathrm{~Hz}, 1 \mathrm{H}), 7.37$ $(\mathrm{dd}, J=14.6,7.6 \mathrm{~Hz}, 3 \mathrm{H}), 7.22(\mathrm{t}, J=8.0 \mathrm{~Hz}, 1 \mathrm{H}), 6.82(\mathrm{dd}, J=17.8,8.0 \mathrm{~Hz}, 2 \mathrm{H})$, $6.49(\mathrm{~d}, J=8.4 \mathrm{~Hz}, 1 \mathrm{H}), 5.93(\mathrm{~d}, J=9.0 \mathrm{~Hz}, 1 \mathrm{H}), 3.81(\mathrm{~s}, 3 \mathrm{H}), 2.66(\mathrm{~s}, 3 \mathrm{H}), 1.42$ (s, 9H). ${ }^{13} \mathrm{C}\{1 \mathrm{H}\} \mathrm{NMR}\left(100 \mathrm{MHz}, \mathrm{CDCl}_{3}\right) \delta 176.9,163.5,1602,155.9,155.1,134.1$, 133.6, 129.8, 125.8, 125.0, 123.7, 120.4, 119.8, 117.8, 111.9, 110.6, 90.8, 79.9, 78.5, 55.8, 41.2, 28.5, 18.7. HRMS (TOFMS-ESI), m/z calcd for $\mathrm{C}_{25} \mathrm{H}_{26} \mathrm{NO}_{5}{ }^{+}\left([\mathrm{M}+\mathrm{H}]^{+}\right)$ 420.1805 , found 420.1813 .

$\mathrm{mV}$

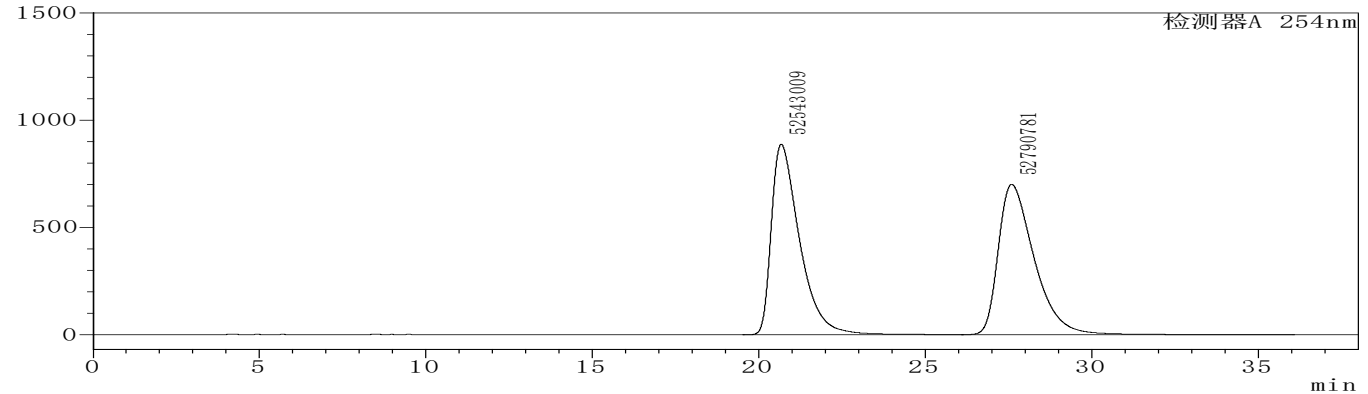

racemic

\begin{tabular}{|c|c|c|c|}
\hline & Retention Time & Area & \%Area \\
\hline 1 & 20.673 & 52543009 & 49.882 \\
\hline 2 & 27.602 & 52790781 & 50.118 \\
\hline
\end{tabular}

$\mathrm{mV}$

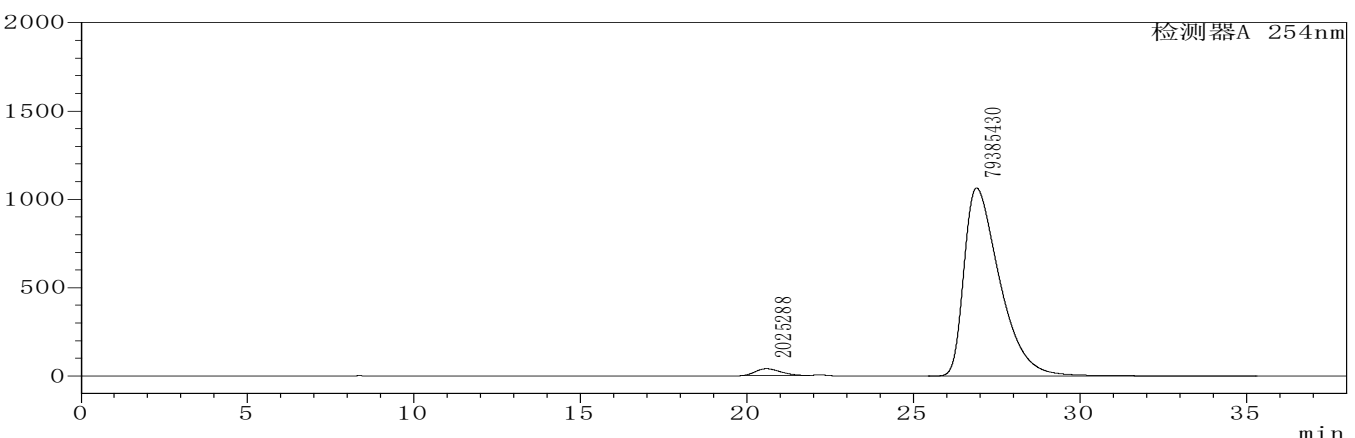

enantio-enriched

\begin{tabular}{|c|c|c|c|}
\hline & Retention Time & Area & \%Area \\
\hline 1 & 20.590 & 2025288 & 2.488 \\
\hline 2 & 26.904 & 79385430 & 97.512 \\
\hline
\end{tabular}


tert-Butyl (S)-(3-(3-(benzyloxy)phenyl)-1-(2-methyl-4-oxo-4H-chromen-3-yl) prop-2-yn-1-yl)carbamate (3q)<smiles>Cc1oc2ccccc2c(=O)c1[C@H](C#Cc1cccc(OCc2ccccc2)c1)NC(C)(C)C</smiles>

Compound 3q: Prepared in $0.2 \mathrm{mmol}$ scale using 1.5 equiv of $1 \mathrm{a}$ at $70{ }^{\circ} \mathrm{C}$ for $64 \mathrm{~h} .78 \%$ yield $(77.4 \mathrm{mg})$, colorless oil, 95\% ee. HPLC (chiral ID column), hexane $/ i-\mathrm{PrOH}=70 / 30$, flow rate $0.8 \mathrm{ml} / \mathrm{min}, \lambda=254$ $\mathrm{nm}, \operatorname{tr}($ minor $)=16.44 \mathrm{~min}, t \mathrm{r}($ major $)=38.58 \mathrm{~min}$. $[\alpha]^{25} \mathrm{D}=+49.2\left(\mathrm{c}=1.0\right.$ in $\left.\mathrm{CH}_{2} \mathrm{Cl}_{2}\right)$.

${ }^{1} \mathrm{H}$ NMR $\left(400 \mathrm{MHz}, \mathrm{CDCl}_{3}\right) \delta 8.21(\mathrm{dd}, J=8.0,1.4 \mathrm{~Hz}, 1 \mathrm{H}), 7.68-7.62(\mathrm{~m}, 1 \mathrm{H})$, $7.44-7.29(\mathrm{~m}, 7 \mathrm{H}), 7.16(\mathrm{t}, J=8.0 \mathrm{~Hz}, 1 \mathrm{H}), 7.03(\mathrm{~d}, J=7.6 \mathrm{~Hz}, 2 \mathrm{H}), 6.92-6.88(\mathrm{~m}$, $1 \mathrm{H}), 6.56(\mathrm{~d}, J=9.2 \mathrm{~Hz}, 1 \mathrm{H}), 5.89(\mathrm{~d}, J=9.4 \mathrm{~Hz}, 1 \mathrm{H}), 5.01(\mathrm{~s}, 2 \mathrm{H}), 2.66(\mathrm{~s}, 3 \mathrm{H}), 1.44$ $(\mathrm{s}, 9 \mathrm{H}) .{ }^{13} \mathrm{C}\{1 \mathrm{H}\} \mathrm{NMR}\left(150 \mathrm{MHz}, \mathrm{CDCl}_{3}\right) \delta 177.1,163.5,158.5,156.0,155.2,136.8$, $133.8,129.3$, 128.7, 128.1, 127.6, 125.8, 125.1, 124.9, 123.9, 123.6, 119.6, 117.9, 117.7, 115.9, 86.8, 82.0, 80.0, 70.1, 41.0, 28.5, 18.6. HRMS (TOFMS-ESI), m/z calcd for $\mathrm{C}_{31} \mathrm{H}_{30} \mathrm{NO}_{5}{ }^{+}\left([\mathrm{M}+\mathrm{H}]^{+}\right)$496.2118, found 496.2122.

$\mathrm{mV}$

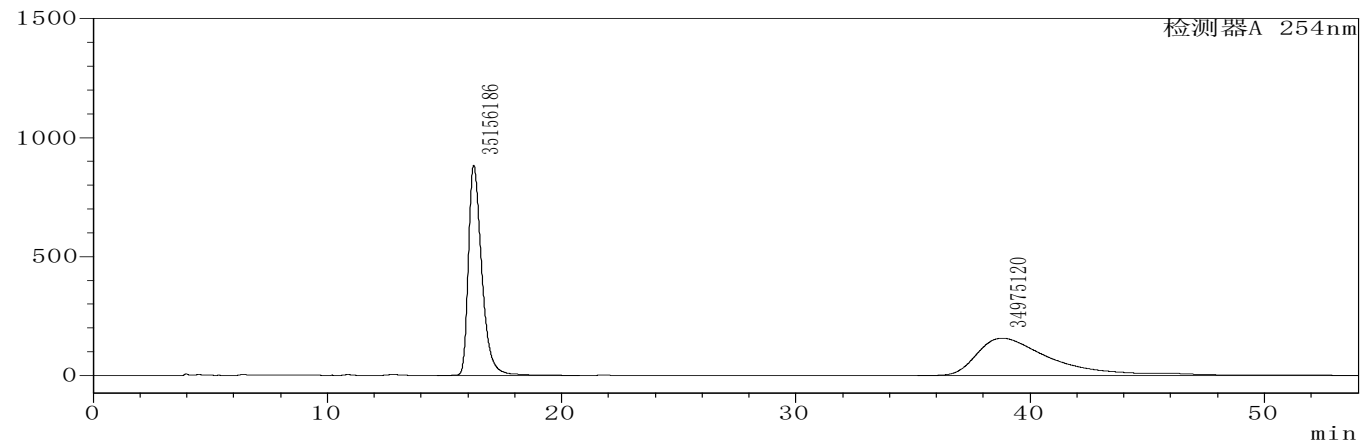

racemic

\begin{tabular}{|c|c|c|c|}
\hline & Retention Time & Area & \%Area \\
\hline 1 & 16.249 & 35156186 & 50.129 \\
\hline 2 & 38.797 & 34975120 & 49.871 \\
\hline
\end{tabular}

$\mathrm{mV}$

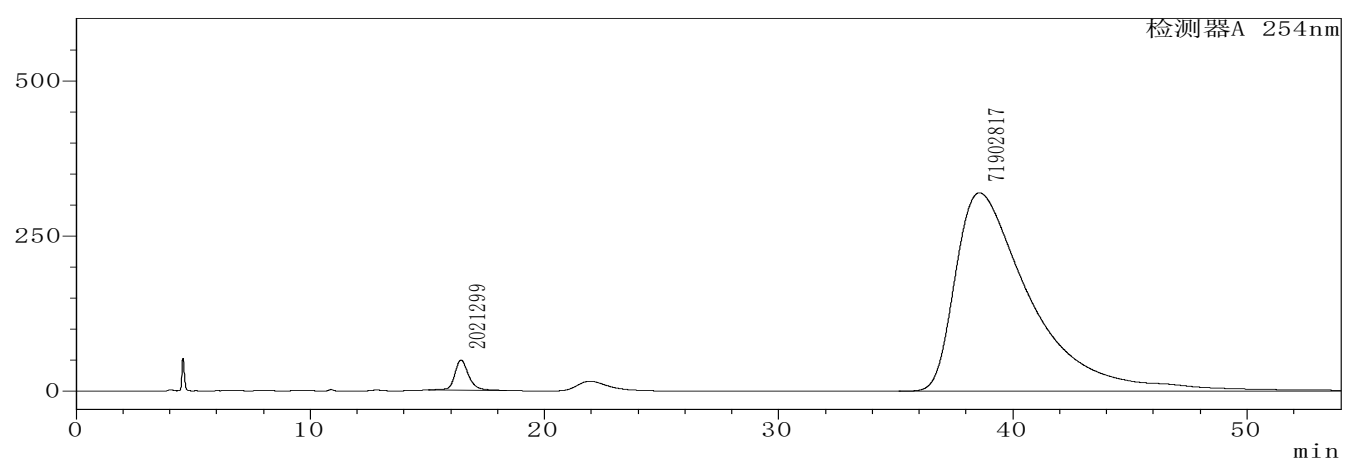

enantio-enriched

\begin{tabular}{|c|c|c|c|}
\hline & Retention Time & Area & \%Area \\
\hline 1 & 16.435 & 1925473 & 2.608 \\
\hline 2 & 38.577 & 71902817 & 97.392 \\
\hline
\end{tabular}


tert-Butyl (S)-(1-(2-methyl-4-oxo-4H-chromen-3-yl)-3-(p-tolyl)prop-2-yn-1-yl) carbamate (3r)<smiles>Cc1ccc(C#C[C@H](NC(=O)OC(C)(C)C)c2c(C)oc3ccccc3c2=O)cc1</smiles>

Compound 3r: Prepared in $0.2 \mathrm{mmol}$ scale using 1.5 equiv of $\mathbf{1 a}$ at $70{ }^{\circ} \mathrm{C}$ for $60 \mathrm{~h} .96 \%$ yield $(77.5 \mathrm{mg})$, colorless oil, 95\% ee. HPLC (chiral ID column), hexane $/ i-\mathrm{PrOH}=70 / 30$, flow rate $0.8 \mathrm{ml} / \mathrm{min}, \lambda=254$ $\mathrm{nm}, t \mathrm{r}($ minor $)=11.06 \mathrm{~min}, \mathrm{tr}($ major $)=18.52 \mathrm{~min}$. $[\alpha]^{25}=+58.1\left(\mathrm{c}=1.0\right.$ in $\left.\mathrm{CH}_{2} \mathrm{Cl}_{2}\right)$.

${ }^{1} \mathrm{H}$ NMR $\left(400 \mathrm{MHz}, \mathrm{CDCl}_{3}\right) \delta 8.20(\mathrm{dd}, J=8.0,1.4 \mathrm{~Hz}, 1 \mathrm{H}), 7.70-7.59(\mathrm{~m}, 1 \mathrm{H})$, $7.39(\mathrm{dd}, J=14.4,7.6 \mathrm{~Hz}, 2 \mathrm{H}), 7.29(\mathrm{~d}, J=8.0 \mathrm{~Hz}, 2 \mathrm{H}), 7.05(\mathrm{~d}, J=8.0 \mathrm{~Hz}, 2 \mathrm{H}), 6.53$ (d, $J=9.0 \mathrm{~Hz}, 1 \mathrm{H}), 5.87(\mathrm{~d}, J=9.4 \mathrm{~Hz}, 1 \mathrm{H}), 2.65(\mathrm{~s}, 3 \mathrm{H}), 2.30(\mathrm{~s}, 3 \mathrm{H}), 1.43(\mathrm{~s}, 9 \mathrm{H})$. ${ }^{13} \mathrm{C}\{1 \mathrm{H}\} \mathrm{NMR}\left(100 \mathrm{MHz}, \mathrm{CDCl}_{3}\right) \delta 177.1,163.4,156.0,155.2,138.4,133.7,131.9$, 129.0, 125.8, 125.1, 123.7, 119.7, 117.9, 86.1, 82.2, 80.0, 41.1, 28.5, 21.6, 18.6. HRMS (TOFMS-ESI), $\mathrm{m} / \mathrm{z}$ calcd for $\mathrm{C}_{25} \mathrm{H}_{26} \mathrm{NO}_{4}{ }^{+}\left([\mathrm{M}+\mathrm{H}]^{+}\right) 404.1856$, found 404.1862.

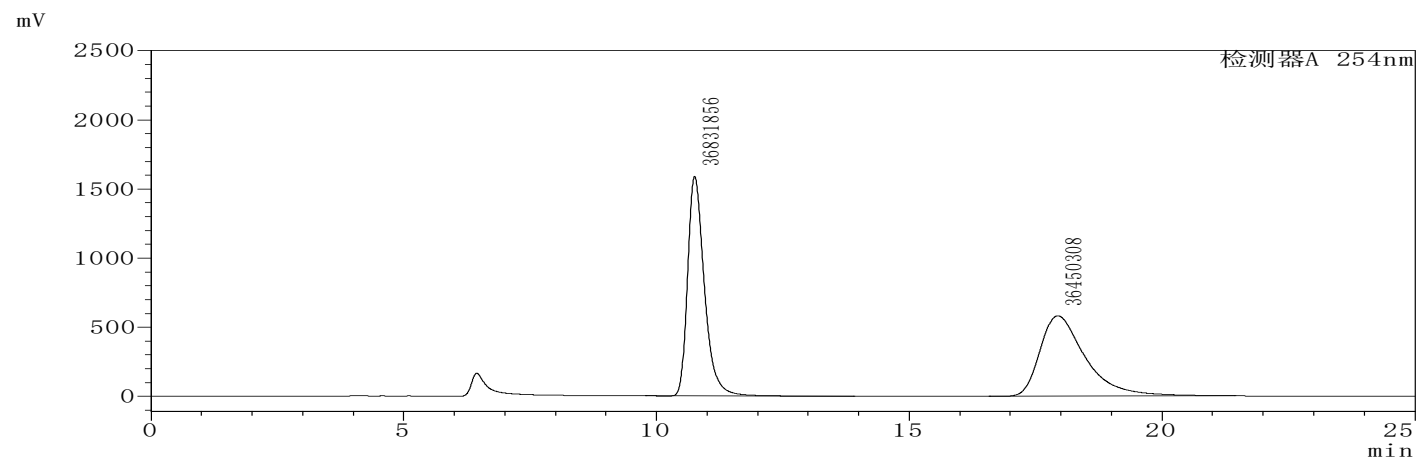

racemic

\begin{tabular}{|c|c|c|c|}
\hline & Retention Time & Area & \%Area \\
\hline 1 & 10.753 & 36831856 & 50.260 \\
\hline 2 & 17.936 & 36450308 & 49.740 \\
\hline
\end{tabular}

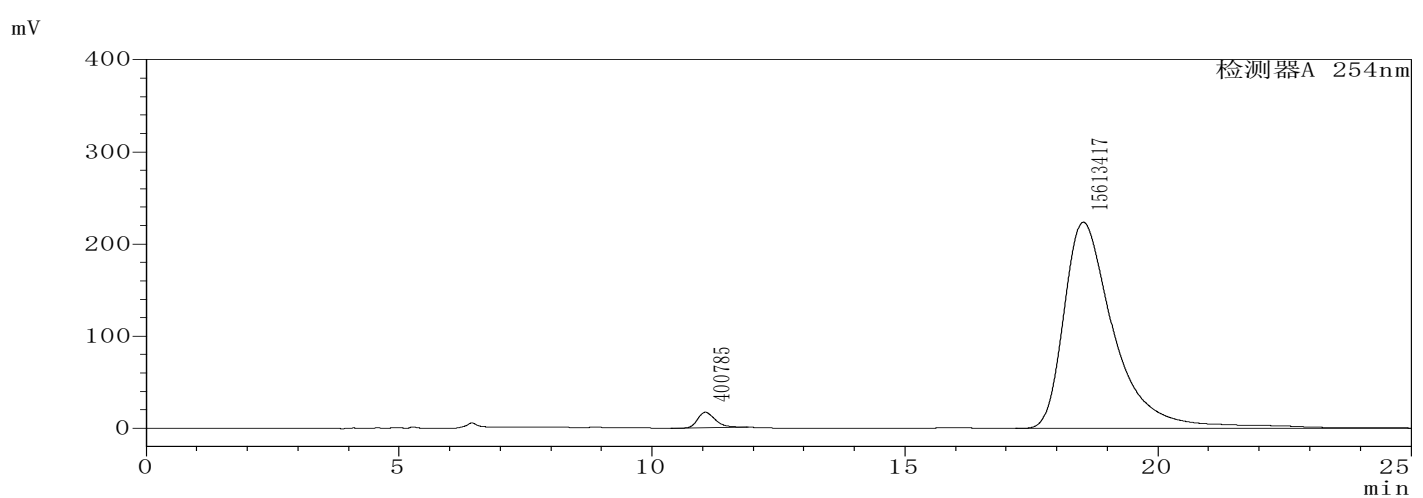

enantio-enriched

\begin{tabular}{|c|c|c|c|}
\hline & Retention Time & Area & \%Area \\
\hline 1 & 11.057 & 400785 & 2.503 \\
\hline 2 & 18.524 & 15613417 & 97.497 \\
\hline
\end{tabular}


tert-Butyl (S)-(3-(4-methoxyphenyl)-1-(2-methyl-4-oxo-4H-chromen-3-yl)prop2-yn-1-yl)carbamate (3s)<smiles>COC(=O)N[C@@H](C#Cc1ccc(OC)cc1)c1c(C)oc2ccccc2c1=O</smiles>

Compound 3s: Prepared in $0.2 \mathrm{mmol}$ scale using 1.5 equiv of $1 \mathrm{a}$ at $70{ }^{\circ} \mathrm{C}$ for 65 h. $95 \%$ yield $(79.7$ $\mathrm{mg}$ ), colorless oil, 94\% ee. HPLC (chiral ID column), hexane $/ i-\mathrm{PrOH}=70 / 30$, flow rate 0.8 $\mathrm{ml} / \mathrm{min}, \lambda=254 \mathrm{~nm}, \mathrm{tr}$ (minor) $=16.40 \mathrm{~min}, \mathrm{tr}$ $($ major $)=30.70 \mathrm{~min}$.

$[\alpha]^{25}=+61.8\left(\mathrm{c}=1.0\right.$ in $\left.\mathrm{CH}_{2} \mathrm{Cl}_{2}\right)$.

${ }^{1} \mathrm{H}$ NMR $\left(400 \mathrm{MHz}, \mathrm{CDCl}_{3}\right) \delta 8.19(\mathrm{~d}, J=8.0 \mathrm{~Hz}, 1 \mathrm{H}), 7.63(\mathrm{t}, J=7.8 \mathrm{~Hz}, 1 \mathrm{H}), 7.41$ $-7.37(\mathrm{~m}, 2 \mathrm{H}), 7.33(\mathrm{~d}, J=8.6 \mathrm{~Hz}, 2 \mathrm{H}), 6.76(\mathrm{~d}, J=8.6 \mathrm{~Hz}, 2 \mathrm{H}), 6.53(\mathrm{~d}, J=9.0 \mathrm{~Hz}$, $1 \mathrm{H}), 5.86(\mathrm{~d}, J=9.2 \mathrm{~Hz}, 1 \mathrm{H}), 3.76(\mathrm{~s}, 3 \mathrm{H}), 2.64(\mathrm{~s}, 3 \mathrm{H}), 1.42(\mathrm{~s}, 9 \mathrm{H}) .{ }^{13} \mathrm{C}\{1 \mathrm{H}\} \mathrm{NMR}$ $\left(100 \mathrm{MHz}, \mathrm{CDCl}_{3}\right) \delta 177.1,163.3,159.6,155.9,155.2,133.7,133.4,125.8,125.1$, 123.6, 119.7, 117.8, 114.8, 113.8, 85.4, 81.9, 79.9, 55.3, 41.0, 28.5, 18.6. HRMS (TOFMS-ESI), m/z calcd for $\mathrm{C}_{25} \mathrm{H}_{26} \mathrm{NO}_{5}^{+}\left([\mathrm{M}+\mathrm{H}]^{+}\right) 420.1805$, found 420.1814 . $\mathrm{mV}$

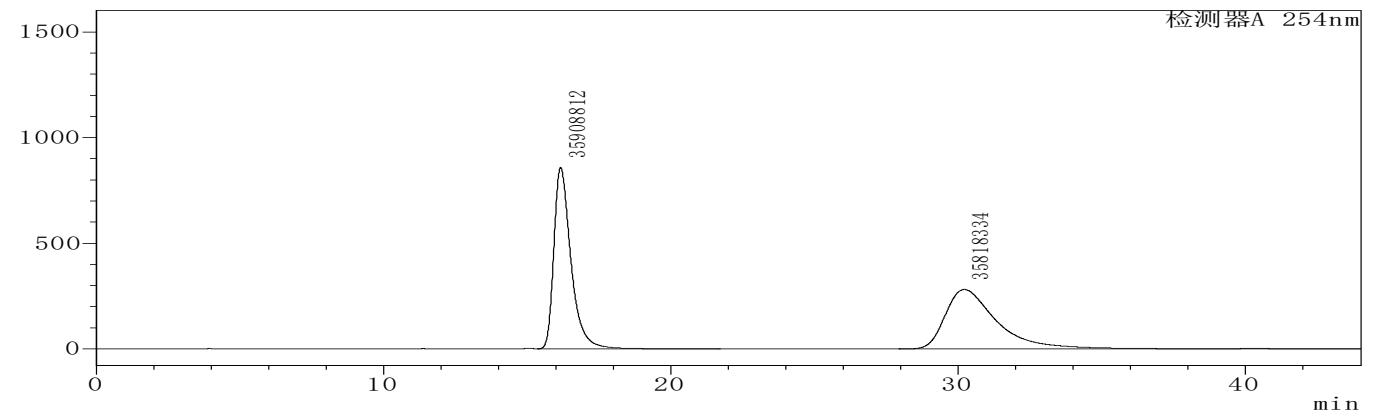

racemic

\begin{tabular}{|c|c|c|c|}
\hline & Retention Time & Area & \%Area \\
\hline 1 & 16.166 & 35908812 & 50.063 \\
\hline 2 & 30.211 & 35818334 & 49.937 \\
\hline
\end{tabular}

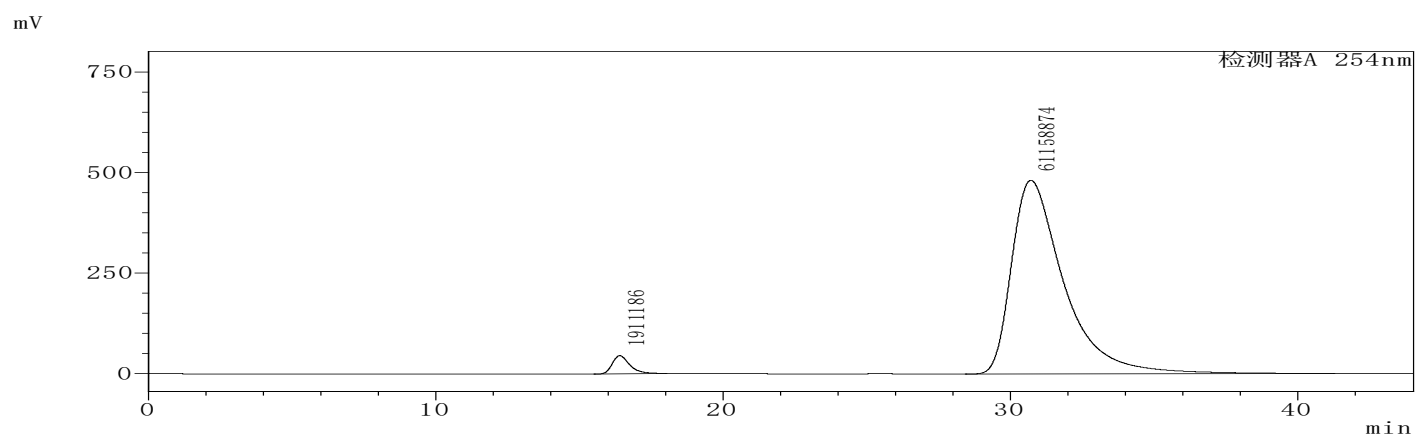

enantio-enriched

\begin{tabular}{|c|c|c|c|}
\hline & Retention Time & Area & \%Area \\
\hline 1 & 16.404 & 1911186 & 3.030 \\
\hline 2 & 30.704 & 61158874 & 96.970 \\
\hline
\end{tabular}


tert-Butyl (S)-(3-(4-chlorophenyl)-1-(2-methyl-4-oxo-4H-chromen-3-yl)prop-2-

yn-1-yl)carbamate (3t)<smiles>CO[R16](=O)NC(C#Cc1ccc(Cl)cc1)c1c(C)oc2ccccc2c1=O</smiles>

Compound 3t: Prepared in $0.2 \mathrm{mmol}$ scale using 1.5 equiv of $1 \mathrm{a}$ at $70{ }^{\circ} \mathrm{C}$ for $60 \mathrm{~h} .79 \%$ yield $(67.0 \mathrm{mg})$, colorless oil, 95\% ee. HPLC (chiral ID column), hexane $/ i-\mathrm{PrOH}=70 / 30$, flow rate $0.8 \mathrm{ml} / \mathrm{min}, \lambda=$ $254 \mathrm{~nm}, \operatorname{tr}($ minor $)=9.10 \mathrm{~min}, \operatorname{tr}($ major $)=13.65 \mathrm{~min}$. $[\alpha]^{25}=+52.0\left(\mathrm{c}=1.0\right.$ in $\left.\mathrm{CH}_{2} \mathrm{Cl}_{2}\right)$.

${ }^{1} \mathrm{H}$ NMR $\left(400 \mathrm{MHz}, \mathrm{CDCl}_{3}\right) \delta 8.19(\mathrm{~d}, J=8.0 \mathrm{~Hz}, 1 \mathrm{H}), 7.65(\mathrm{t}, J=7.8 \mathrm{~Hz}, 1 \mathrm{H}), 7.39$ $(\mathrm{dd}, J=13.6,7.8 \mathrm{~Hz}, 2 \mathrm{H}), 7.32(\mathrm{~d}, J=8.6 \mathrm{~Hz}, 2 \mathrm{H}), 7.21(\mathrm{~d}, J=8.4 \mathrm{~Hz}, 2 \mathrm{H}), 6.53(\mathrm{~d}$, $J=9.2 \mathrm{~Hz}, 1 \mathrm{H}), 5.86(\mathrm{~d}, J=9.4 \mathrm{~Hz}, 1 \mathrm{H}), 2.64(\mathrm{~s}, 3 \mathrm{H}), 1.43(\mathrm{~s}, 9 \mathrm{H}) .{ }^{13} \mathrm{C}\{1 \mathrm{H}\} \mathrm{NMR}$ $\left(100 \mathrm{MHz}, \mathrm{CDCl}_{3}\right) \delta 177.1,163.5,155.9,155.2,134.3,133.8,133.2,128.5,125.8$, $125.2,123.6,121.3,119.4,117.9,87.9,80.9,80.1,41.0,28.5,18.6$. HRMS (TOFMS-ESI), $\mathrm{m} / \mathrm{z}$ calcd for $\mathrm{C}_{24} \mathrm{H}_{23} \mathrm{ClNO}_{4}{ }^{+}\left([\mathrm{M}+\mathrm{H}]^{+}\right) 424.1310$, found 424.1317 .

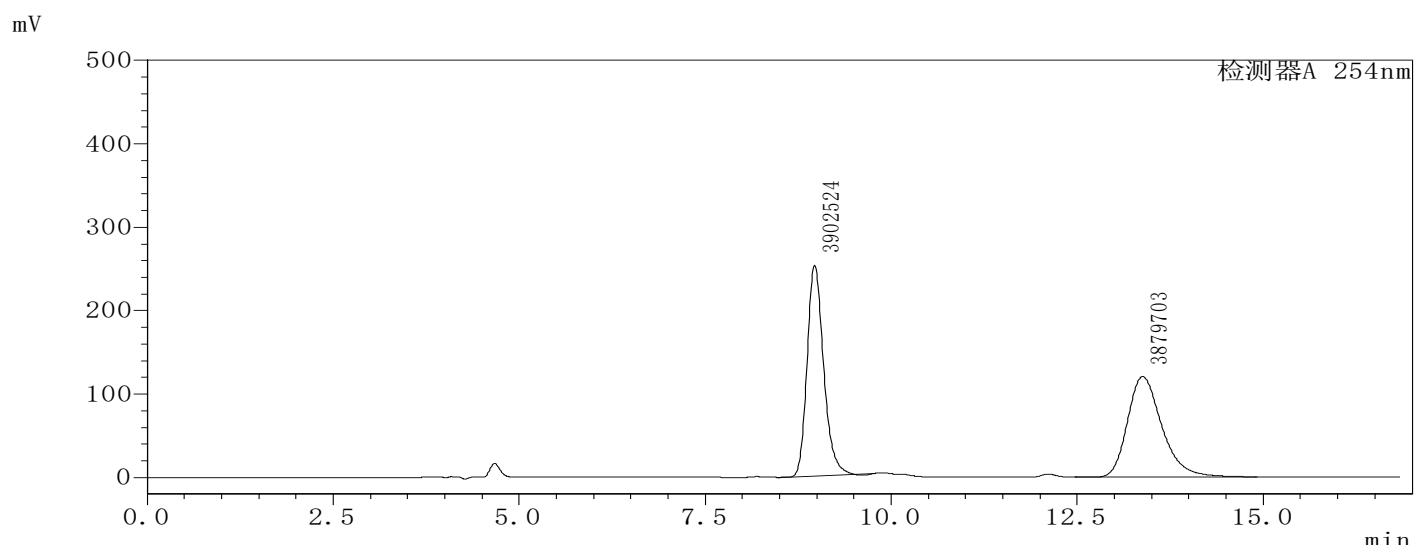

racemic

\begin{tabular}{|c|c|c|c|}
\hline & Retention Time & Area & \%Area \\
\hline 1 & 8.971 & 3902524 & 50.147 \\
\hline 2 & 13.378 & 3879703 & 49.853 \\
\hline
\end{tabular}

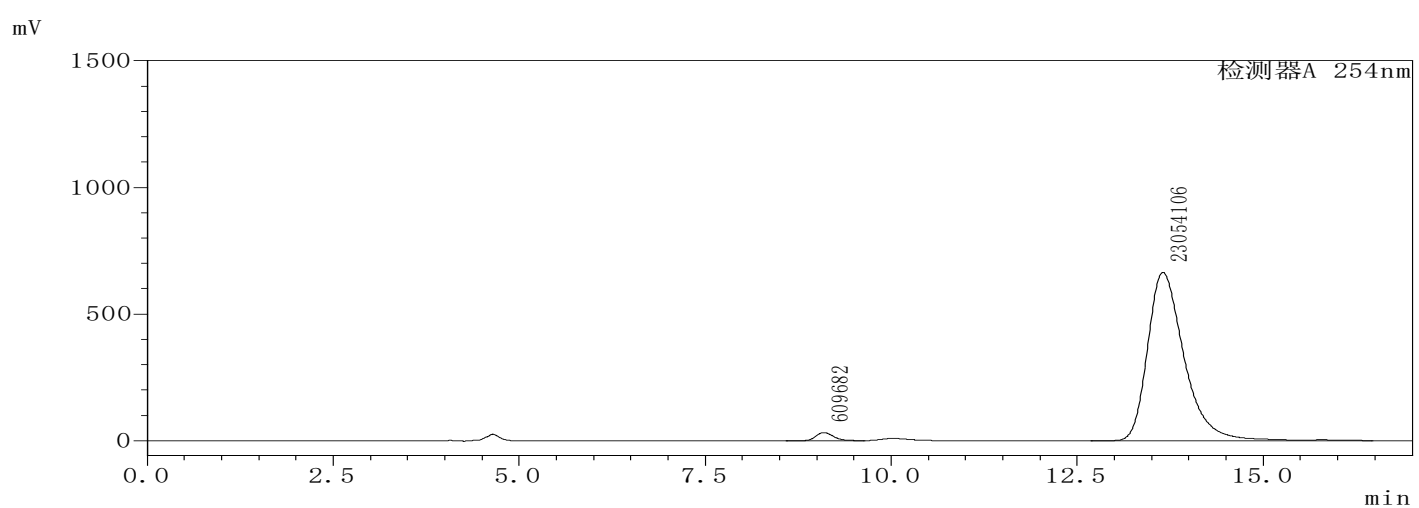

enantio-enriched

\begin{tabular}{|c|c|c|c|}
\hline & Retention Time & Area & \%Area \\
\hline 1 & 9.095 & 609682 & 2.576 \\
\hline 2 & 13.651 & 23054106 & 97.424 \\
\hline
\end{tabular}


tert-Butyl (S)-(3-(4-fluorophenyl)-1-(2-methyl-4-oxo-4H-chromen-3-yl)prop-2yn-1-yl)carbamate (3u)<smiles>Cc1oc2ccccc2c(=O)c1C(C#Cc1ccc(F)cc1)NC(=O)OC(C)(C)C</smiles>

Compound 3u: Prepared in $0.2 \mathrm{mmol}$ scale using 1.5 equiv of $1 \mathrm{a}$ at $70{ }^{\circ} \mathrm{C}$ for $70 \mathrm{~h} .97 \%$ yield $(80.0 \mathrm{mg})$, colorless oil, 95\% ee. HPLC (chiral ID column), hexane $/ i-\mathrm{PrOH}=70 / 30$, flow rate $0.8 \mathrm{ml} / \mathrm{min}, \lambda=254$ $\mathrm{nm}, t \mathrm{r}$ (minor) $=9.04 \mathrm{~min}, t \mathrm{r}$ (major) $=14.00 \mathrm{~min}$. $[\alpha]^{25} \mathrm{D}=+48.6\left(\mathrm{c}=1.0\right.$ in $\left.\mathrm{CH}_{2} \mathrm{Cl}_{2}\right)$.

${ }^{1} \mathrm{H}$ NMR $\left(400 \mathrm{MHz}, \mathrm{CDCl}_{3}\right) \delta 8.19(\mathrm{~d}, J=8.0 \mathrm{~Hz}, 1 \mathrm{H}), 7.72-7.59(\mathrm{~m}, 1 \mathrm{H}), 7.50-$ $7.33(\mathrm{~m}, 4 \mathrm{H}), 6.94(\mathrm{t}, J=8.6 \mathrm{~Hz}, 2 \mathrm{H}), 6.53(\mathrm{~d}, J=9.2 \mathrm{~Hz}, 1 \mathrm{H}), 5.85(\mathrm{~d}, J=9.4 \mathrm{~Hz}$, $1 \mathrm{H}), 2.65(\mathrm{~s}, 3 \mathrm{H}), 1.43(\mathrm{~s}, 9 \mathrm{H}) .{ }^{13} \mathrm{C}\{1 \mathrm{H}\} \mathrm{NMR}\left(150 \mathrm{MHz}, \mathrm{CDCl}_{3}\right) \delta 177.1,163.4(\mathrm{~d}, J$ $=10.2 \mathrm{~Hz}), 161.8,156.0,155.2,133.9(\mathrm{~d}, J=8.4 \mathrm{~Hz}), 133.8,125.8,125.2,123.7$, $119.6,118.9$ (d, $J=3.4 \mathrm{~Hz}), 117.9,115.5(\mathrm{~d}, J=22.2 \mathrm{~Hz}), 86.6,81.0,80.1,41.0,28.5$, 18.6. HRMS (TOFMS-ESI), $\mathrm{m} / \mathrm{z}$ calcd for $\mathrm{C}_{24} \mathrm{H}_{23} \mathrm{FNO}_{4}{ }^{+}\left([\mathrm{M}+\mathrm{H}]^{+}\right) 408.1606$, found 408.1615.

$\mathrm{mV}$

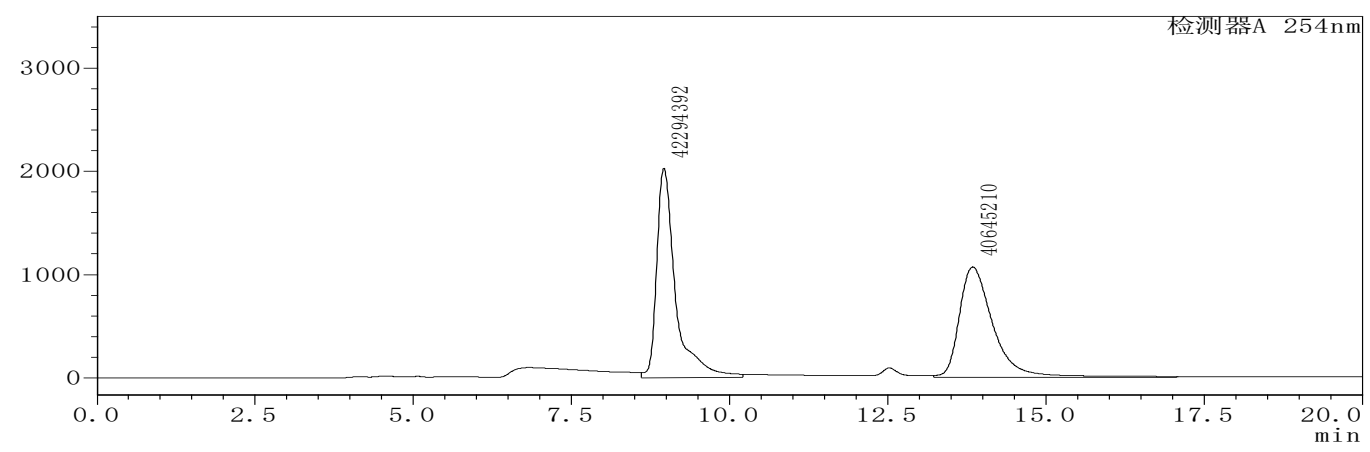

racemic

\begin{tabular}{|c|c|c|c|}
\hline & Retention Time & Area & \%Area \\
\hline 1 & 8.961 & 42294392 & 50.994 \\
\hline 2 & 13.843 & 40645210 & 49.006 \\
\hline
\end{tabular}

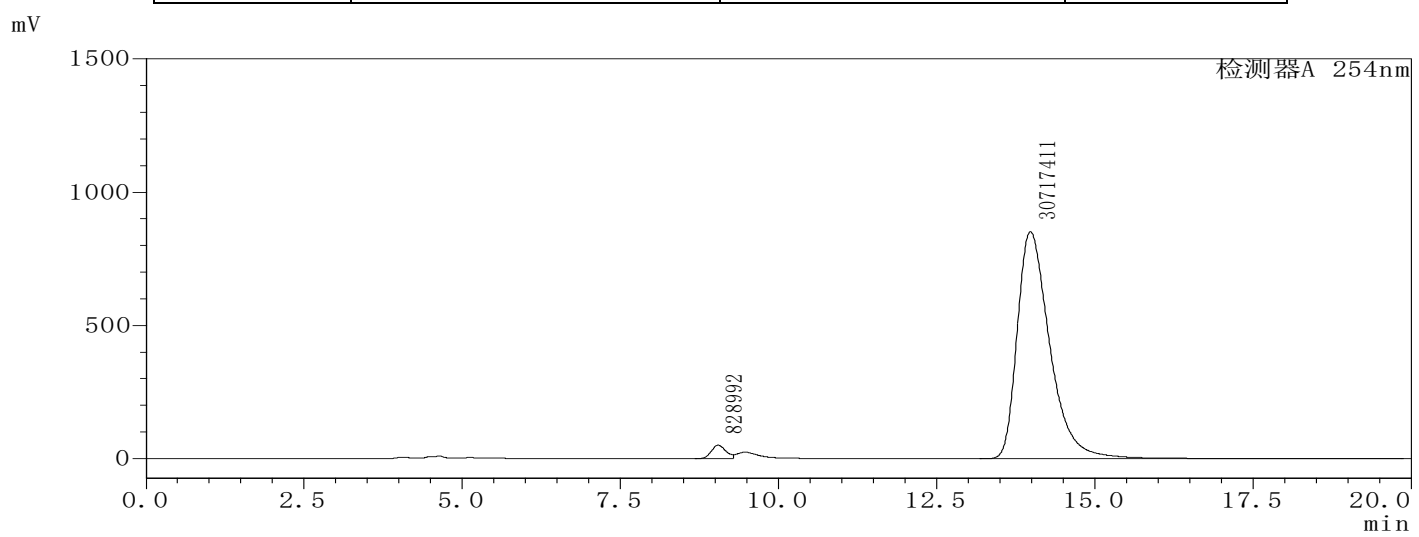

enantio-enriched

\begin{tabular}{|c|c|c|c|}
\hline & Retention Time & Area & \%Area \\
\hline 1 & 9.043 & 828992 & 2.628 \\
\hline 2 & 13.983 & 30717411 & 97.372 \\
\hline
\end{tabular}


tert-Butyl (S)-(1-(2-methyl-4-oxo-4H-chromen-3-yl)-3-(naphthalen-2-yl)prop-2-

yn-1-yl)carbamate (3v)<smiles>Cc1oc2ccccc2c(=O)c1[C@H](C#Cc1ccc2ccccc2c1)C(=O)OC(C)(C)C</smiles>

Compound 3v: Prepared in $0.2 \mathrm{mmol}$ scale using 1.5 equiv of $1 \mathrm{a}$ at $70{ }^{\circ} \mathrm{C}$ for $62 \mathrm{~h} .64 \%$ yield $(56.5 \mathrm{mg})$, colorless oil, 94\% ee. HPLC (chiral ID column), hexane $/ i-\mathrm{PrOH}=70 / 30$, flow rate $0.8 \mathrm{ml} / \mathrm{min}, \lambda=254$ $\mathrm{nm}, t \mathrm{r}($ minor $)=14.76 \mathrm{~min}, t \mathrm{r}($ major $)=24.67 \mathrm{~min}$.

$[\alpha]^{25} \mathrm{D}=+48.5\left(\mathrm{c}=1.0\right.$ in $\left.\mathrm{CH}_{2} \mathrm{Cl}_{2}\right)$.

${ }^{1} \mathrm{H}$ NMR $\left(400 \mathrm{MHz}, \mathrm{CDCl}_{3}\right) \delta 8.23(\mathrm{~d}, J=8.0 \mathrm{~Hz}, 1 \mathrm{H}), 7.94(\mathrm{~s}, 1 \mathrm{H}), 7.80-7.70(\mathrm{~m}$, $3 \mathrm{H}), 7.69-7.62(\mathrm{~m}, 1 \mathrm{H}), 7.50-7.37(\mathrm{~m}, 5 \mathrm{H}), 6.59(\mathrm{~d}, J=9.0 \mathrm{~Hz}, 1 \mathrm{H}), 5.94(\mathrm{~d}, J=$ $9.4 \mathrm{~Hz}, 1 \mathrm{H}), 2.68(\mathrm{~s}, 3 \mathrm{H}), 1.45(\mathrm{~s}, 9 \mathrm{H}) .{ }^{13} \mathrm{C}\{1 \mathrm{H}\} \mathrm{NMR}\left(150 \mathrm{MHz}, \mathrm{CDCl}_{3}\right) \delta 177.2$, $163.5,156.0,155.2,133.8,133.0,132.9,132.0,128.8,127.9,127.8,127.8,126.7$, $126.5,125.9,125.2,123.7,120.2,119.7,117.9,87.2,82.4,80.1,41.2,28.5,18.7$. HRMS (FTMS-ESI), m/z calcd for $\mathrm{C}_{28} \mathrm{H}_{25} \mathrm{NNaO}_{4}{ }^{+}\left([\mathrm{M}+\mathrm{Na}]^{+}\right) 462.1676$, found 462.1673 .

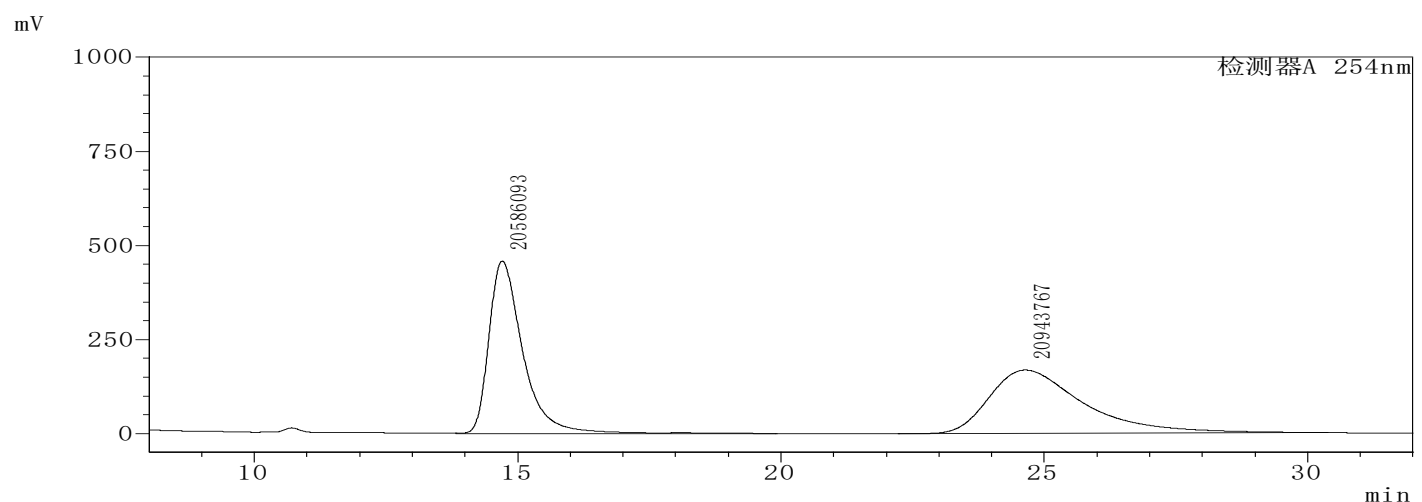

racemic

\begin{tabular}{|c|c|c|c|}
\hline & Retention Time & Area & \%Area \\
\hline 1 & 14.709 & 20586093 & 49.596 \\
\hline 2 & 24.636 & 20943767 & 50.431 \\
\hline
\end{tabular}

$\mathrm{mV}$

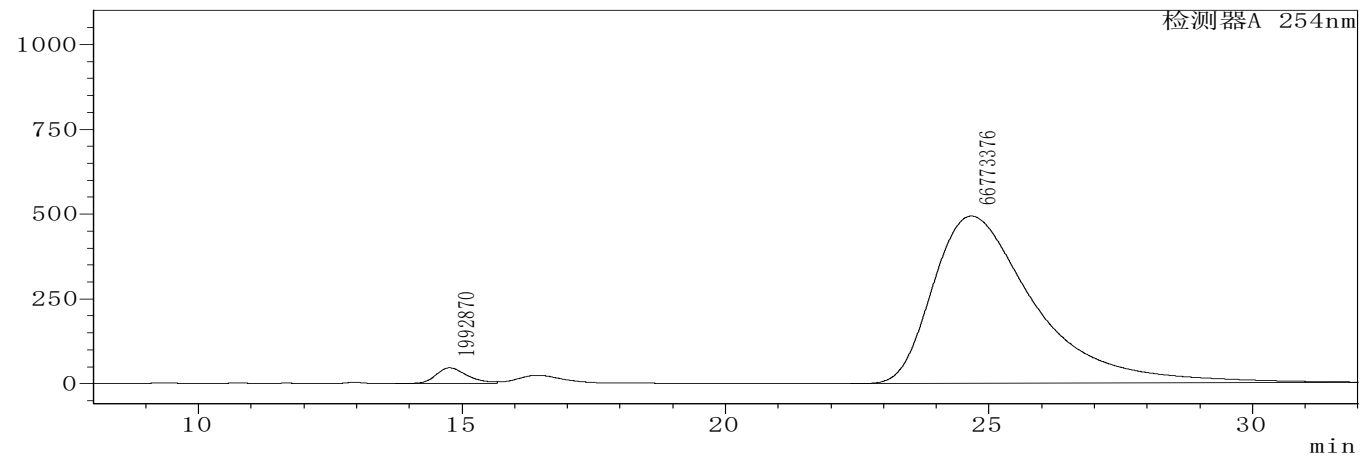

enantio-enriched

\begin{tabular}{|c|c|c|c|}
\hline & Retention Time & Area & \%Area \\
\hline 1 & 14.762 & 1992870 & 2.898 \\
\hline 2 & 24.666 & 66773376 & 97.102 \\
\hline
\end{tabular}


tert-Butyl (S)-(3-cyclohexyl-1-(2-methyl-4-oxo-4H-chromen-3-yl)prop-2-yn-1-yl) carbamate (3w)<smiles>Cc1oc2ccccc2c(=O)c1[C@H](C#CC1CCCCC1)NC(=O)O</smiles>

Compound 3w: Prepared in $0.2 \mathrm{mmol}$ scale using 1.5 equiv of $1 \mathrm{a}$ at $70{ }^{\circ} \mathrm{C}$ for $65 \mathrm{~h} .86 \%$ yield $(69.0 \mathrm{mg})$, colorless oil, $86 \%$ ee. HPLC (chiral ID column), hexane $/ i-\mathrm{PrOH}=70 / 30$, flow rate $0.8 \mathrm{ml} / \mathrm{min}, \lambda=254$ $\mathrm{nm}, \mathrm{tr}($ minor $)=7.09 \mathrm{~min}, \mathrm{tr}($ major $)=9.25 \mathrm{~min}$.

$[\alpha]^{25} \mathrm{D}=+29.7\left(\mathrm{c}=1.0\right.$ in $\left.\mathrm{CH}_{2} \mathrm{Cl}_{2}\right)$.

${ }^{1} \mathrm{H}$ NMR $\left(400 \mathrm{MHz}, \mathrm{CDCl}_{3}\right) \delta 8.16(\mathrm{dd}, J=8.0,1.4 \mathrm{~Hz}, 1 \mathrm{H}), 7.69-7.52(\mathrm{~m}, 1 \mathrm{H})$, $7.46-7.31(\mathrm{~m}, 2 \mathrm{H}), 6.36(\mathrm{~d}, J=9.0 \mathrm{~Hz}, 1 \mathrm{H}), 5.61(\mathrm{~d}, J=9.0 \mathrm{~Hz}, 1 \mathrm{H}), 2.58(\mathrm{~s}, 3 \mathrm{H})$, $2.36-2.25(\mathrm{~m}, 1 \mathrm{H}), 1.77-1.67(\mathrm{~m}, 2 \mathrm{H}), 1.66-1.58(\mathrm{~m}, 2 \mathrm{H}), 1.51-1.30(\mathrm{~m}, 12 \mathrm{H})$, $1.29-1.12(\mathrm{~m}, 3 \mathrm{H}) .{ }^{13} \mathrm{C}\{1 \mathrm{H}\} \mathrm{NMR}\left(100 \mathrm{MHz}, \mathrm{CDCl}_{3}\right) \delta 176.9,163.1,155.8,155.1$, 133.5, 125.8, 124.9, 123.7, 120.1, 117.8, 86.7, 79.7, 77.4, 40.6, 32.6, 29.1, 28.4, 25.9, 24.9, 18.5. HRMS (FTMS-ESI), $\mathrm{m} / \mathrm{z}$ calcd for $\mathrm{C}_{24} \mathrm{H}_{29} \mathrm{NNaO}_{4}{ }^{+}\left([\mathrm{M}+\mathrm{Na}]^{+}\right) 418.1989$, found 418.1996 .

$\mathrm{mV}$

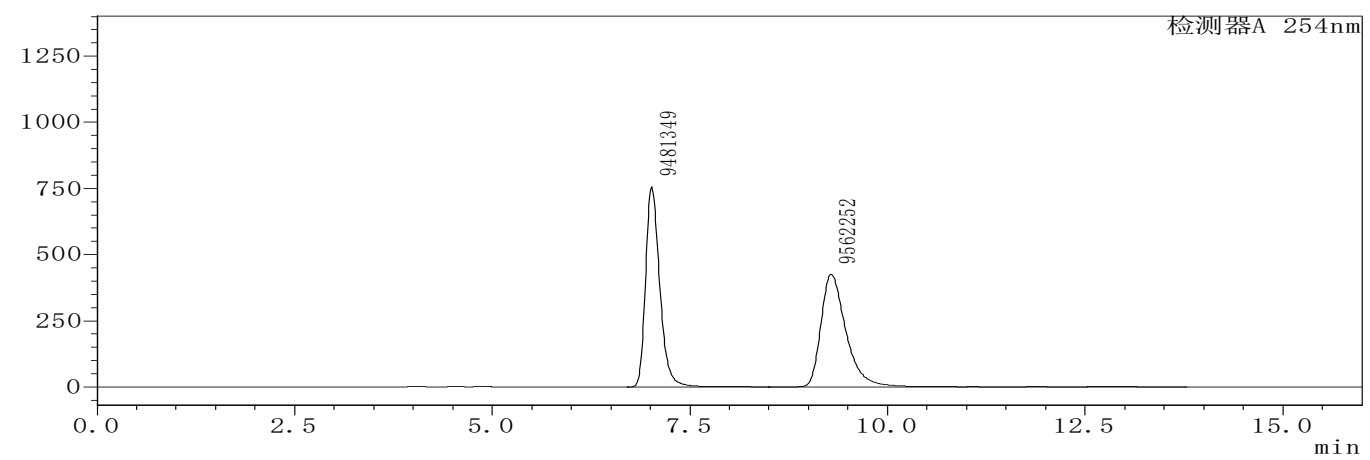

racemic

\begin{tabular}{|c|c|c|c|}
\hline & Retention Time & Area & \%Area \\
\hline 1 & 7.017 & 9481349 & 49.788 \\
\hline 2 & 9.287 & 9562252 & 50.212 \\
\hline
\end{tabular}

$\mathrm{mV}$

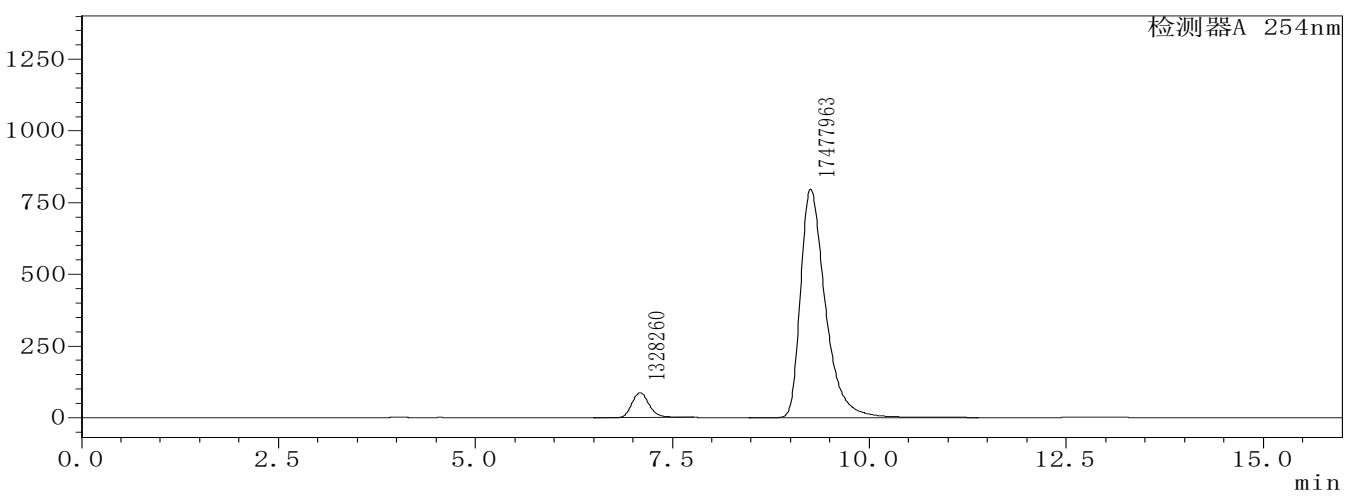

enantio-enriched

\begin{tabular}{|c|c|c|c|}
\hline & Retention Time & Area & \%Area \\
\hline 1 & 7.090 & 1328260 & 7.063 \\
\hline 2 & 9.254 & 17477963 & 92.937 \\
\hline
\end{tabular}


tert-Butyl (S)-(1-(2-methyl-4-oxo-4H-chromen-3-yl)-5-phenylpent-2-yn-1-yl)carbamate (3x)<smiles>Cc1oc2ccccc2c(=O)c1[C@H](C#CCCc1ccccc1)C(=O)OC(C)(C)C</smiles>

Compound 3x: Prepared in $0.2 \mathrm{mmol}$ scale using 1.5 equiv of $1 \mathrm{a}$ at $70{ }^{\circ} \mathrm{C}$ for 65 h. $70 \%$ yield (58.8 $\mathrm{mg}$ ), colorless oil, 93\% ee. HPLC (chiral ID column), hexane $/ i-\mathrm{PrOH}=70 / 30$, flow rate 0.8 $\mathrm{ml} / \mathrm{min}, \lambda=254 \mathrm{~nm}, t \mathrm{r}$ (minor) $=10.55 \mathrm{~min}, t \mathrm{r}$

$($ major $)=14.04 \mathrm{~min}$.

$[\alpha]^{25}=+33.9\left(\mathrm{c}=1.0\right.$ in $\left.\mathrm{CH}_{2} \mathrm{Cl}_{2}\right)$.

${ }^{1} \mathrm{H}$ NMR $\left(400 \mathrm{MHz}, \mathrm{CDCl}_{3}\right) \delta 8.20(\mathrm{~d}, J=7.8 \mathrm{~Hz}, 1 \mathrm{H}), 7.65(\mathrm{t}, J=7.8 \mathrm{~Hz}, 1 \mathrm{H}), 7.44$ $-7.36(\mathrm{~m}, 2 \mathrm{H}), 7.23-7.10(\mathrm{~m}, 5 \mathrm{H}), 6.45(\mathrm{~d}, J=9.2 \mathrm{~Hz}, 1 \mathrm{H}), 5.61(\mathrm{~d}, J=9.4 \mathrm{~Hz}, 1 \mathrm{H})$, $2.78(\mathrm{t}, J=7.6 \mathrm{~Hz}, 2 \mathrm{H}), 2.55(\mathrm{~s}, 3 \mathrm{H}), 2.44(\mathrm{t}, J=6.8 \mathrm{~Hz}, 2 \mathrm{H}), 1.42(\mathrm{~s}, 9 \mathrm{H}) .{ }^{13} \mathrm{C}\{1 \mathrm{H}\}$ NMR $\left(100 \mathrm{MHz}, \mathrm{CDCl}_{3}\right) \delta 177.2,163.2,155.9,155.2,140.8,133.7,128.6,128.3$, 126.2, 125.8, 125.1, 123.7, 119.8, 117.8, 81.9, 79.8, 78.5, 40.6, 35.0, 28.5, 21.2, 18.5. HRMS (FTMS-ESI), m/z calcd for $\mathrm{C}_{26} \mathrm{H}_{27} \mathrm{NNaO}_{4}{ }^{+}\left([\mathrm{M}+\mathrm{Na}]^{+}\right) 440.1832$, found 440.1838 .

$\mathrm{mV}$

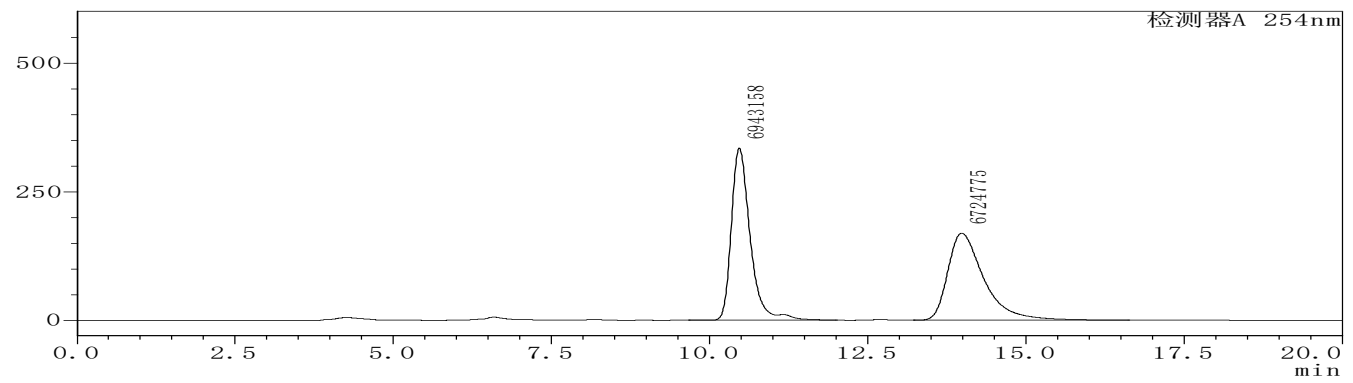

racemic

\begin{tabular}{|c|c|c|c|}
\hline & Retention Time & Area & \%Area \\
\hline 1 & 10.469 & 6943158 & 50.799 \\
\hline 2 & 13.988 & 6724775 & 49.201 \\
\hline
\end{tabular}

$\mathrm{mV}$

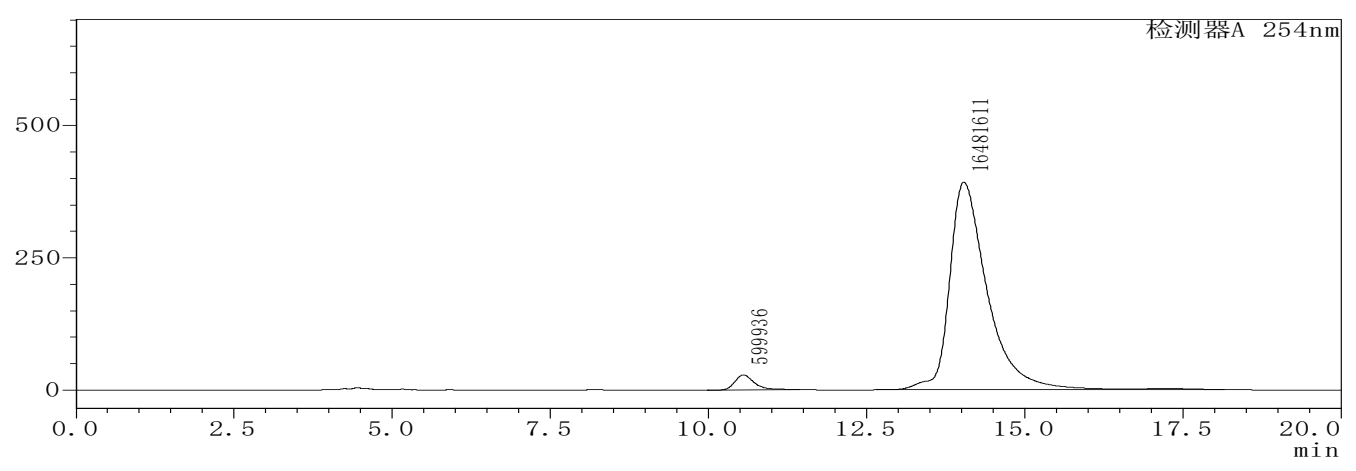

enantio-enriched

\begin{tabular}{|c|c|c|c|}
\hline & Retention Time & Area & \%Area \\
\hline 1 & 10.552 & 599936 & 3.512 \\
\hline 2 & 14.039 & 16481611 & 96.488 \\
\hline
\end{tabular}


tert-Butyl (R)-(1-(2-methyl-4-oxo-4H-chromen-3-yl)-3-(trimethylsilyl)prop-2-yn1-yl)carbamate (3y)<smiles>Cc1oc2ccccc2c(=O)c1[C@H](C#CC#N)NC(=O)OC(C)(C)C</smiles>

Compound 3y: Prepared in $0.2 \mathrm{mmol}$ scale using 1.5 equiv of $1 \mathrm{a}$ at $70{ }^{\circ} \mathrm{C}$ for $64 \mathrm{~h} .71 \%$ yield $(54.5 \mathrm{mg})$, colorless oil, 95\% ee. HPLC (chiral ID column), hexane $/ i-\mathrm{PrOH}=95 / 5$, flow rate $0.8 \mathrm{ml} / \mathrm{min}, \lambda=254 \mathrm{~nm}, t \mathrm{r}($ minor $)=8.69 \mathrm{~min}, t \mathrm{r}$

$($ major $)=10.14 \mathrm{~min}$.

$[\alpha]^{25}{ }_{D}=+34.0\left(\mathrm{c}=1.0\right.$ in $\left.\mathrm{CH}_{2} \mathrm{Cl}_{2}\right)$.

${ }^{1} \mathrm{H}$ NMR $\left(400 \mathrm{MHz}, \mathrm{CDCl}_{3}\right) \delta 8.19(\mathrm{~d}, J=7.0 \mathrm{~Hz}, 1 \mathrm{H}), 7.66-7.61(\mathrm{~m}, 1 \mathrm{H}), 7.44-$ $7.34(\mathrm{~m}, 2 \mathrm{H}), 6.38(\mathrm{~d}, J=8.2 \mathrm{~Hz}, 1 \mathrm{H}), 5.66(\mathrm{~d}, J=9.2 \mathrm{~Hz}, 1 \mathrm{H}), 2.60$ (s, 3H), 1.41 (s, 9H), $0.12(\mathrm{~s}, 9 \mathrm{H}) .{ }^{13} \mathrm{C}\{1 \mathrm{H}\} \mathrm{NMR}\left(150 \mathrm{MHz}, \mathrm{CDCl}_{3}\right) \delta 176.8,163.5,155.9,155.1$, 133.7, 125.9, 125.1, 123.7, 119.5, 117.8, 102.7, 86.7, 80.0, 41.1, 28.5, 18.6, 0.0. HRMS (TOFMS-ESI), m/z calcd for $\mathrm{C}_{21} \mathrm{H}_{28} \mathrm{NO}_{4} \mathrm{Si}^{+}\left([\mathrm{M}+\mathrm{H}]^{+}\right) 386.1782$, found 386.1791 .

$\mathrm{mV}$

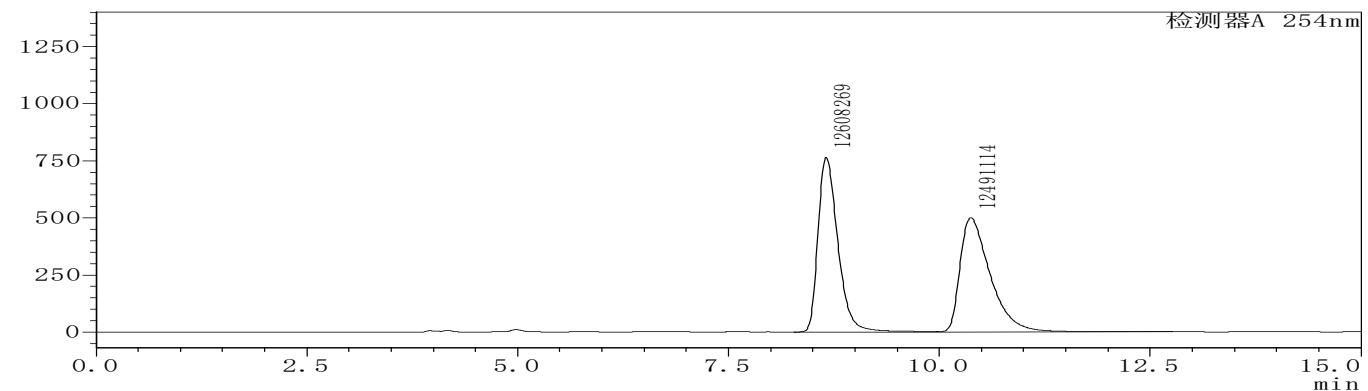

racemic

\begin{tabular}{|c|c|c|c|}
\hline & Retention Time & Area & \%Area \\
\hline 1 & 8.658 & 12608269 & 50.233 \\
\hline 2 & 10.373 & 12491114 & 49.767 \\
\hline
\end{tabular}

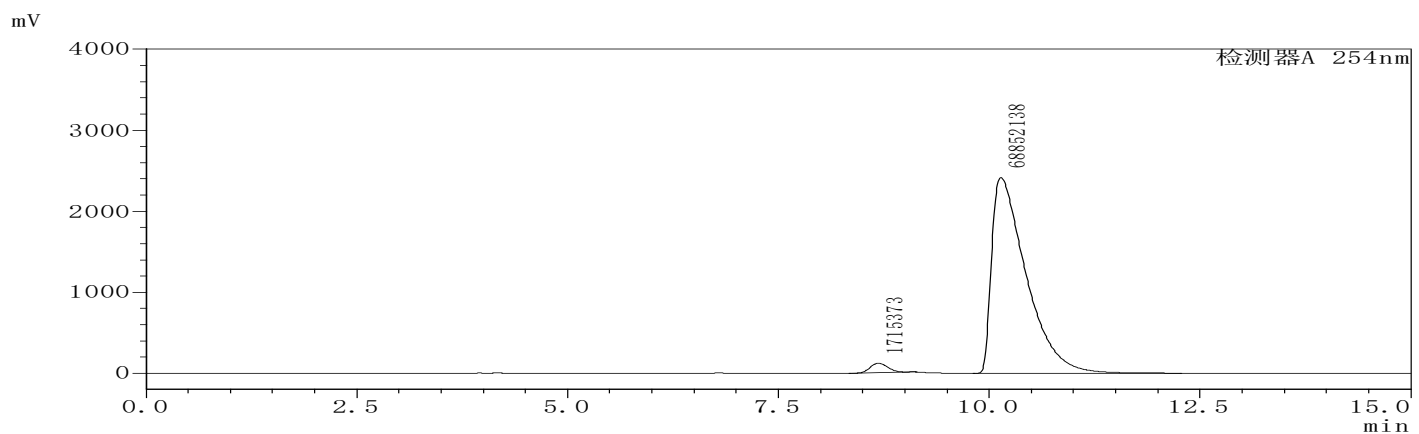

enantio-enriched

\begin{tabular}{|c|c|c|c|}
\hline & Retention Time & Area & \%Area \\
\hline 1 & 8.687 & 1715373 & 2.431 \\
\hline 2 & 10.140 & 68852138 & 97.569 \\
\hline
\end{tabular}


tert-Butyl $(S, E)-(1-(4-0 x 0-2-s t y r y l-4 H$-chromen-3-yl)-3-phenylprop-2-yn-1-yl) carbamate (4a)<smiles>CC(C)(C)OC(=O)[C@H](C#Cc1c(C=Cc2ccccc2)oc2ccccc2c1=O)c1ccccc1</smiles>

Compound 4a: Prepared in $0.2 \mathrm{mmol}$ scale using 1.2 equiv of $1 \mathrm{a}$ at $70{ }^{\circ} \mathrm{C}$ for 51 h. $90 \%$ yield $(86.0 \mathrm{mg})$, yellow solid; mp: $108.6-110.3{ }^{\circ} \mathrm{C}, 96 \%$ ee. HPLC (chiral ID column), hexane $/ i-\mathrm{PrOH}=70 / 30$, flow rate $0.8 \mathrm{ml} / \mathrm{min}, \lambda=254 \mathrm{~nm}, t \mathrm{r}$ $($ minor $)=11.97 \mathrm{~min}, t \mathrm{r}($ major $)=16.68 \mathrm{~min}$.

$[\alpha]^{25} \mathrm{D}=-184.6\left(\mathrm{c}=1.0\right.$ in $\left.\mathrm{CH}_{2} \mathrm{Cl}_{2}\right)$.

${ }^{1} \mathrm{H}$ NMR $\left(400 \mathrm{MHz}, \mathrm{cdcl}_{3}\right) \delta 8.21(\mathrm{~d}, J=8.0 \mathrm{~Hz}, 1 \mathrm{H}), 7.74(\mathrm{~d}, J=16.2 \mathrm{~Hz}, 1 \mathrm{H}), 7.70$ $-7.66(\mathrm{~m}, 3 \mathrm{H}), 7.56-7.47(\mathrm{~m}, 2 \mathrm{H}), 7.46-7.35(\mathrm{~m}, 6 \mathrm{H}), 7.29-7.20(\mathrm{~m}, 3 \mathrm{H}), 6.67(\mathrm{~d}$, $J=7.4 \mathrm{~Hz}, 1 \mathrm{H}), 6.22(\mathrm{~d}, J=9.2 \mathrm{~Hz}, 1 \mathrm{H}), 1.46(\mathrm{~s}, 9 \mathrm{H}) .{ }^{13} \mathrm{C}\{1 \mathrm{H}\}$ NMR $(150 \mathrm{MHz}$, $\left.\mathrm{CDCl}_{3}\right) \delta 177.7,158.2,155.5,155.2,139.3,135.2,134.1,132.0,130.2,129.1,128.3$, 128.2, 128.2, 125.8, 125.0, 123.6, 122.8, 118.6, 117.8, 116.7, 87.3, 82.4, 80.1, 40.2, 28.5. HRMS (FTMS-ESI), $\mathrm{m} / \mathrm{z}$ calcd for $\mathrm{C}_{31} \mathrm{H}_{27} \mathrm{NNaO}_{4}{ }^{+}\left([\mathrm{M}+\mathrm{Na}]^{+}\right) 500.1832$, found 500.1836 .

$\mathrm{mV}$

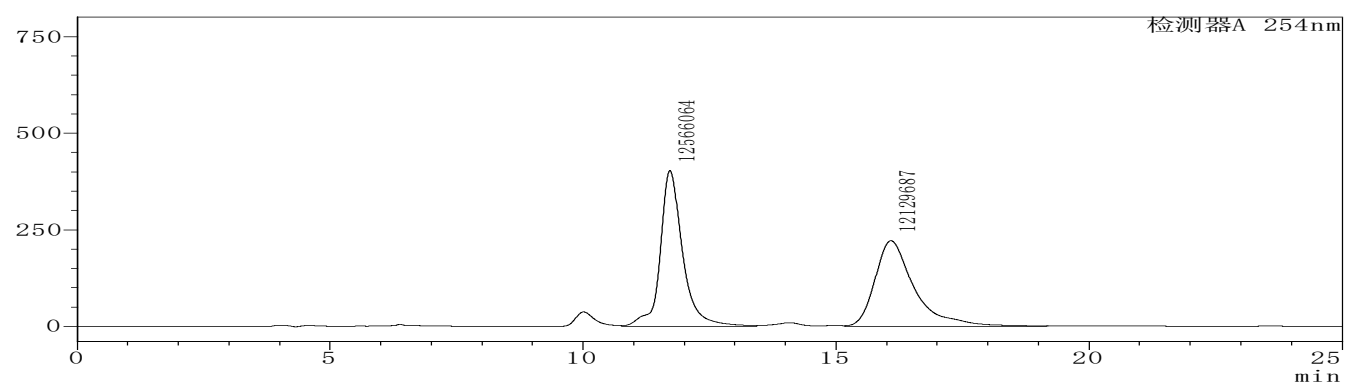

racemic

\begin{tabular}{|c|c|c|c|}
\hline & Retention Time & Area & \%Area \\
\hline 1 & 11.719 & 12566064 & 50.884 \\
\hline 2 & 16.084 & 12129687 & 49.116 \\
\hline
\end{tabular}

$\mathrm{mV}$

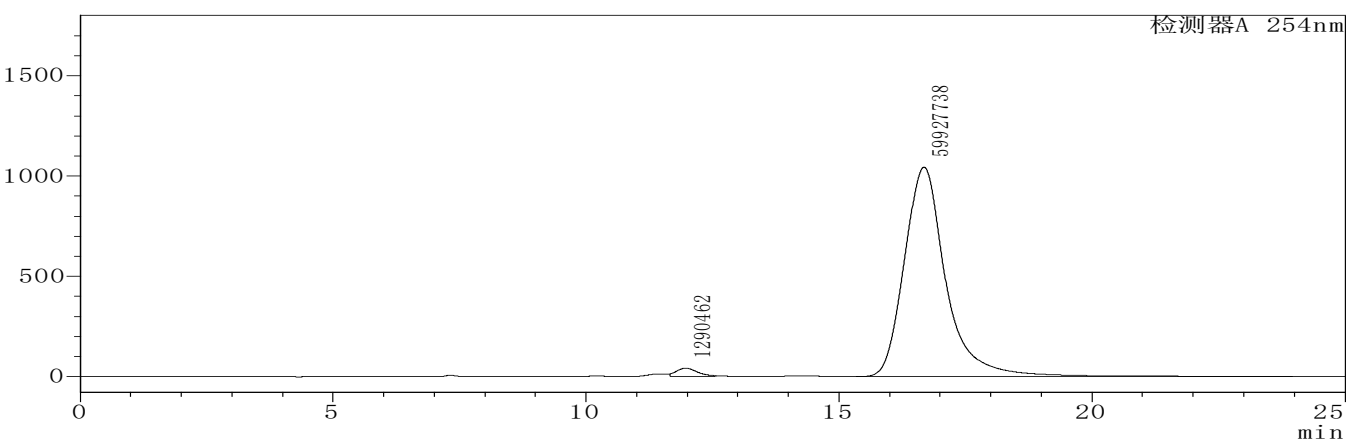

enantio-enriched

\begin{tabular}{|c|c|c|c|}
\hline & Retention Time & Area & \%Area \\
\hline 1 & 11.967 & 1290462 & 2.108 \\
\hline 2 & 16.680 & 59927738 & 97.892 \\
\hline
\end{tabular}


tert-Butyl (S,E)-(1-(2-(2-fluorostyryl)-4-oxo-4H-chromen-3-yl)-3-phenylprop-2yn-1-yl)carbamate (4b)<smiles>CC(C)(C)OC(=O)C[C@H](C#Cc1c(/C=C/c2ccccc2F)oc2ccccc2c1=O)c1ccccc1</smiles>

Compound 4b: Prepared in $0.2 \mathrm{mmol}$ scale using 1.2 equiv of $1 \mathbf{a}$ at $70{ }^{\circ} \mathrm{C}$ for $72 \mathrm{~h} .84 \%$ yield $(83.0 \mathrm{mg}$ ), yellow solid; mp: $137.3-138.8{ }^{\circ} \mathrm{C}, 96 \%$ ee. HPLC (chiral ID column), hexane $/ i-\mathrm{PrOH}=70 / 30$, flow rate $0.8 \mathrm{ml} / \mathrm{min}, \lambda=254 \mathrm{~nm}, t \mathrm{r}$ $($ minor $)=9.56 \mathrm{~min}, \mathrm{tr}($ major $)=13.73 \mathrm{~min}$.

$[\alpha]^{25} \mathrm{D}=-162.3\left(\mathrm{c}=1.0\right.$ in $\left.\mathrm{CH}_{2} \mathrm{Cl}_{2}\right)$.

${ }^{1} \mathrm{H}$ NMR $\left(400 \mathrm{MHz}, \mathrm{CDCl}_{3}\right) \delta 8.21(\mathrm{~d}, J=8.0 \mathrm{~Hz}, 1 \mathrm{H}), 7.91$ $(\mathrm{d}, J=15.8 \mathrm{~Hz}, 1 \mathrm{H}), 7.79-7.74(\mathrm{~m}, 1 \mathrm{H}), 7.72-7.68(\mathrm{~m}, 1 \mathrm{H}), 7.58-7.52(\mathrm{~m}, 2 \mathrm{H})$, $7.44-7.33(\mathrm{~m}, 4 \mathrm{H}), 7.26-7.18(\mathrm{~m}, 4 \mathrm{H}), 7.16-7.10(\mathrm{~m}, 1 \mathrm{H}), 6.64(\mathrm{~d}, J=8.0 \mathrm{~Hz}$, $1 \mathrm{H}), 6.21(\mathrm{~d}, J=9.2 \mathrm{~Hz}, 1 \mathrm{H}), 1.45(\mathrm{~s}, 9 \mathrm{H}) .{ }^{13} \mathrm{C}\{1 \mathrm{H}\} \mathrm{NMR}\left(150 \mathrm{MHz}, \mathrm{CDCl}_{3}\right) \delta 177.7$, $161.3(\mathrm{~d}, J=253.0 \mathrm{~Hz}), 157.9,155.5,155.2,134.2,132.0,131.6(\mathrm{~d}, J=8.8 \mathrm{~Hz})$, $131.4(\mathrm{~d}, J=4.0 \mathrm{~Hz}), 128.4,128.2,125.9,125.2,124.7(\mathrm{~d}, J=3.4 \mathrm{~Hz}), 123.7,123.4$ $(\mathrm{d}, J=11.6 \mathrm{~Hz}), 122.8,119.2,118.9,117.9,116.3(\mathrm{~d}, J=21.8 \mathrm{~Hz}), 87.2,82.5,80.1$, 40.2, 28.5. HRMS (ESI), $\mathrm{m} / \mathrm{z}$ calcd for $\mathrm{C}_{31} \mathrm{H}_{26} \mathrm{FNNaO}_{4}{ }^{+}\left([\mathrm{M}+\mathrm{Na}]^{+}\right) 518.1738$, found 518.1747 .

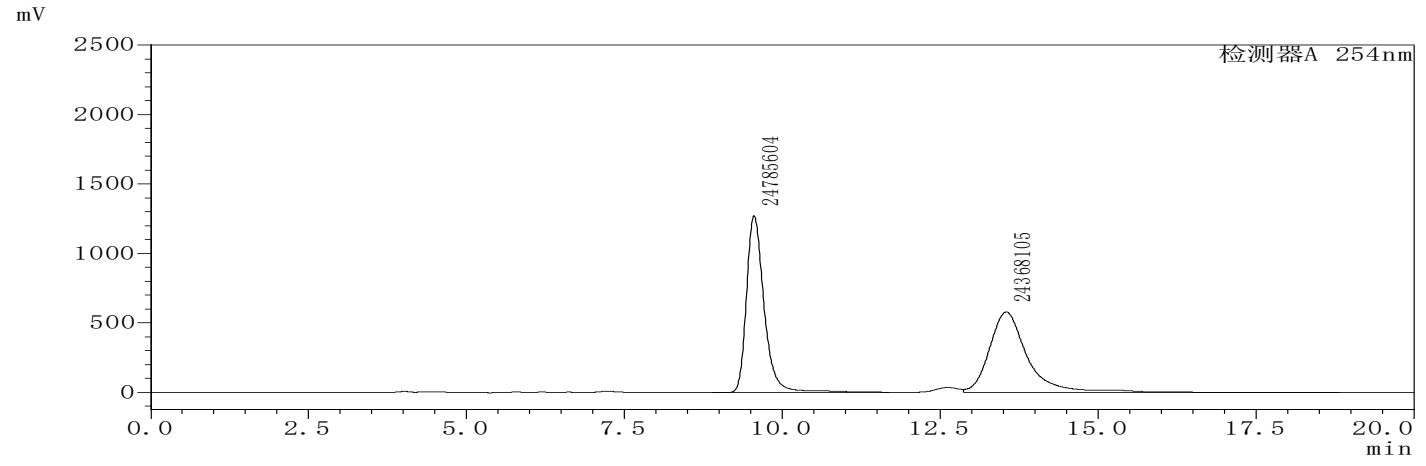

racemic

\begin{tabular}{|c|c|c|c|}
\hline & Retention Time & Area & \%Area \\
\hline 1 & 9.553 & 24785604 & 50.425 \\
\hline 2 & 13.547 & 24368105 & 49.575 \\
\hline
\end{tabular}

$\mathrm{mV}$

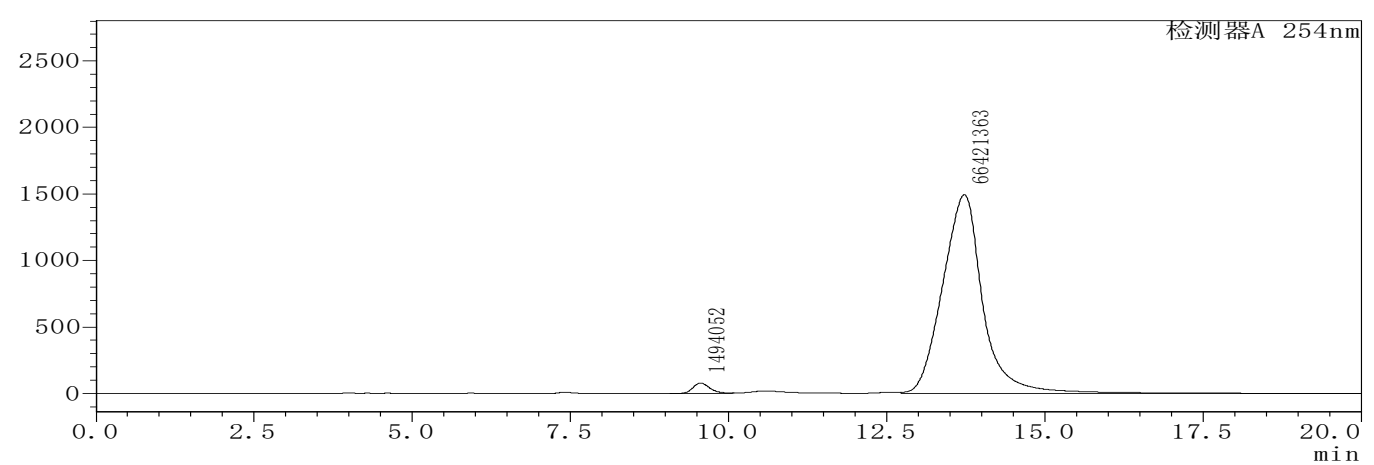

enantio-enriched

\begin{tabular}{|c|c|c|c|}
\hline & Retention Time & Area & \%Area \\
\hline 1 & 9.557 & 1494052 & 2.200 \\
\hline 2 & 13.729 & 66421363 & 97.800 \\
\hline
\end{tabular}


tert-Butyl $(S, E)$-(1-(2-(2-methoxystyryl)-4-oxo-4H-chromen-3-yl)-3-phenylprop2-yn-1-yl)carbamate (4c)<smiles>COC(=O)[C@@H](C#Cc1ccccc1)c1c(/C=C/c2ccccc2OC)oc2ccccc2c1=O</smiles>

Compound 4c: Prepared in $0.2 \mathrm{mmol}$ scale using 1.2 equiv of $1 \mathrm{a}$ at $70{ }^{\circ} \mathrm{C}$ for $48 \mathrm{~h} .83 \%$ yield $(84.3 \mathrm{mg}$ ), yellow solid; mp: $75.1-76.8{ }^{\circ} \mathrm{C}, 95 \%$ ee. HPLC (chiral ID column), hexane $/ i-\mathrm{PrOH}=70 / 30$, flow rate $0.8 \mathrm{ml} / \mathrm{min}, \lambda=254 \mathrm{~nm}, t \mathrm{r}$ $($ minor $)=15.78 \mathrm{~min}, t \mathrm{r}$ (major) $=21.86 \mathrm{~min}$.

$[\alpha]^{25}=-74.1\left(\mathrm{c}=1.0\right.$ in $\left.\mathrm{CH}_{2} \mathrm{Cl}_{2}\right)$.

${ }^{1} \mathrm{H}$ NMR $\left(400 \mathrm{MHz}, \mathrm{CDCl}_{3}\right) \delta 8.22(\mathrm{dd}, J=8.0,1.2 \mathrm{~Hz}, 1 \mathrm{H})$, $8.06(\mathrm{~d}, J=15.8 \mathrm{~Hz}, 1 \mathrm{H}), 7.76-7.65(\mathrm{~m}, 2 \mathrm{H}), 7.57(\mathrm{t}, J=11.0 \mathrm{~Hz}, 2 \mathrm{H}), 7.45-7.34$ (m, 4H), $7.29-7.20(\mathrm{~m}, 3 \mathrm{H}), 7.03(\mathrm{t}, J=7.6 \mathrm{~Hz}, 1 \mathrm{H}), 6.95(\mathrm{~d}, J=8.4 \mathrm{~Hz}, 1 \mathrm{H}), 6.67$ $(\mathrm{d}, J=8.0 \mathrm{~Hz}, 1 \mathrm{H}), 6.23(\mathrm{~d}, J=9.2 \mathrm{~Hz}, 1 \mathrm{H}), 3.96(\mathrm{~s}, 3 \mathrm{H}), 1.45(\mathrm{~s}, 9 \mathrm{H}) .{ }^{13} \mathrm{C}\{1 \mathrm{H}\}$ NMR $\left(100 \mathrm{MHz}, \mathrm{CDCl}_{3}\right) \delta 177.8,159.0,158.3,155.5,155.1,134.7,133.9,132.0$, 131.5, 128.8, 128.3, 128.2, 125.8, 125.0, 124.3, 123.6, 122.9, 121.0, 118.3, 117.9, 117.4, 111.2, 87.5, 82.2, 80.0, 55.7, 40.1, 28.5. HRMS (FTMS-ESI), m/z calcd for $\mathrm{C}_{32} \mathrm{H}_{29} \mathrm{NNaO}_{5}{ }^{+}\left([\mathrm{M}+\mathrm{Na}]^{+}\right) 530.1938$, found 530.1940 .

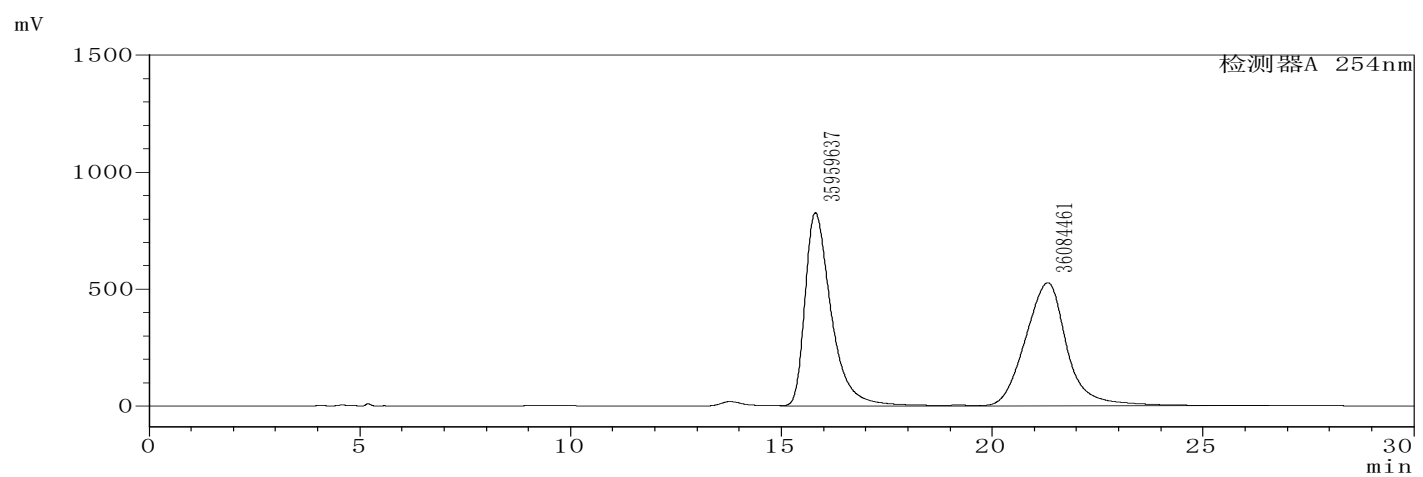

racemic

\begin{tabular}{|c|c|c|c|}
\hline & Retention Time & Area & \%Area \\
\hline 1 & 15.806 & 35959637 & 49.913 \\
\hline 2 & 21.317 & 36084461 & 50.087 \\
\hline
\end{tabular}

$\mathrm{mV}$

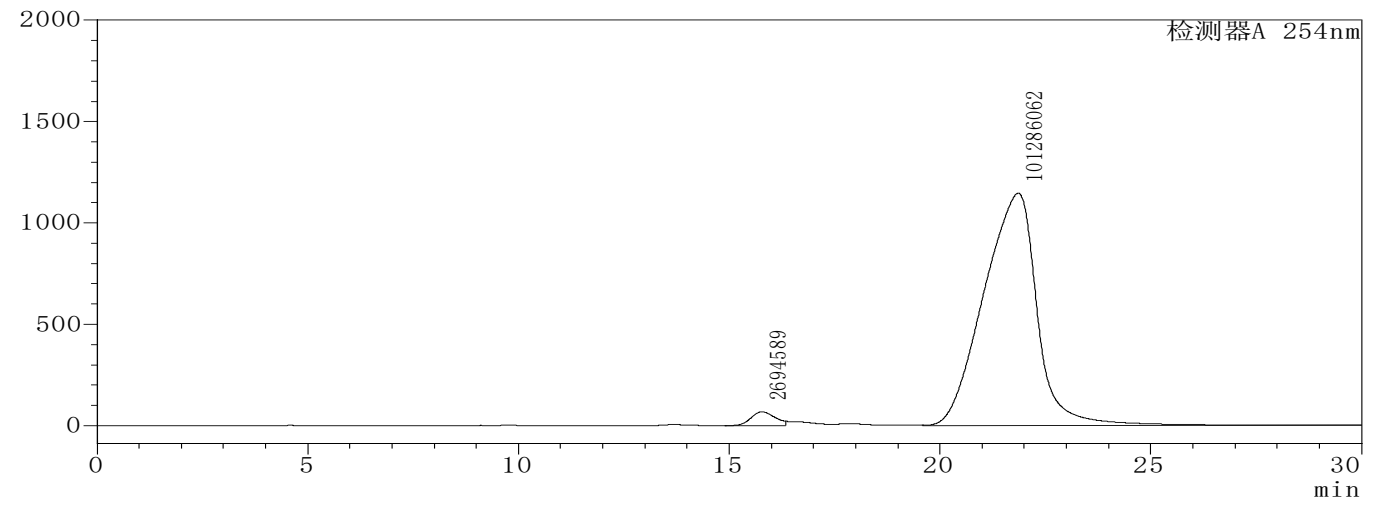

enantio-enriched

\begin{tabular}{|c|c|c|c|}
\hline & Retention Time & Area & \%Area \\
\hline 1 & 15.777 & 2694589 & 2.591 \\
\hline 2 & 21.857 & 101286062 & 97.409 \\
\hline
\end{tabular}


tert-Butyl $(S, E)$-(1-(2-(4-methylstyryl)-4-oxo-4H-chromen-3-yl)-3-phenylprop-2yn-1-yl)carbamate (4d)<smiles>Cc1ccc(/C=C/c2oc3ccccc3c(=O)c2C(C#Cc2ccccc2)NC(=O)OC(C)(C)C)cc1</smiles>

Compound 4d: Prepared in $0.2 \mathrm{mmol}$ scale using 1.2 equiv of $1 \mathrm{a}$ at $70{ }^{\circ} \mathrm{C}$ for $72 \mathrm{~h} .94 \%$ yield (93.0 mg), yellow solid; mp: 83.1-85.7 ${ }^{\circ} \mathrm{C}, 97 \%$ ee. HPLC (chiral ID column), hexane $/ i-\mathrm{PrOH}=70 / 30$, flow rate $0.8 \mathrm{ml} / \mathrm{min}, \lambda=254 \mathrm{~nm}$, $\operatorname{tr}($ minor $)=10.94 \mathrm{~min}, t \mathrm{r}$ (major $)=14.96 \mathrm{~min}$.

$[\alpha]^{25} \mathrm{D}=-193.2\left(\mathrm{c}=1.0\right.$ in $\left.\mathrm{CH}_{2} \mathrm{Cl}_{2}\right)$.

${ }^{1} \mathrm{H} \mathrm{NMR}\left(400 \mathrm{MHz}, \mathrm{CDCl}_{3}\right) \delta 8.17(\mathrm{~d}, J=7.8 \mathrm{~Hz}, 1 \mathrm{H})$, $7.70-7.64(\mathrm{~m}, 2 \mathrm{H}), 7.54(\mathrm{~d}, J=7.8 \mathrm{~Hz}, 2 \mathrm{H}), 7.49(\mathrm{~d}, J=8.4 \mathrm{~Hz}, 1 \mathrm{H}), 7.42(\mathrm{dd}, J=$ $7.2,3.4 \mathrm{~Hz}, 2 \mathrm{H}), 7.39-7.33(\mathrm{~m}, 2 \mathrm{H}), 7.26-7.23(\mathrm{~m}, 3 \mathrm{H}), 7.19(\mathrm{~d}, J=7.8 \mathrm{~Hz}, 2 \mathrm{H})$, $6.74(\mathrm{~d}, J=8.2 \mathrm{~Hz}, 1 \mathrm{H}), 6.24(\mathrm{~d}, J=9.2 \mathrm{~Hz}, 1 \mathrm{H}), 2.36(\mathrm{~s}, 3 \mathrm{H}), 1.47(\mathrm{~s}, 9 \mathrm{H}) .{ }^{13} \mathrm{C}\{1 \mathrm{H}\}$ NMR $\left(150 \mathrm{MHz}, \mathrm{CDCl}_{3}\right) \delta 177.7,158.5,155.5,155.2,140.7,139.4,134.0,132.6$, 132.0, 129.9, 128.3, 128.2, 125.9, 125.0, 123.7, 122.9, 118.4, 117.8, 115.7, 87.4, 82.4, 80.1, 40.2, 28.6, 21.6. HRMS (FTMS-ESI), m/z calcd for $\mathrm{C}_{32} \mathrm{H}_{29} \mathrm{NNaO}_{4}{ }^{+}\left([\mathrm{M}+\mathrm{Na}]^{+}\right)$ 514.1989 , found 514.1996.

$\mathrm{mV}$

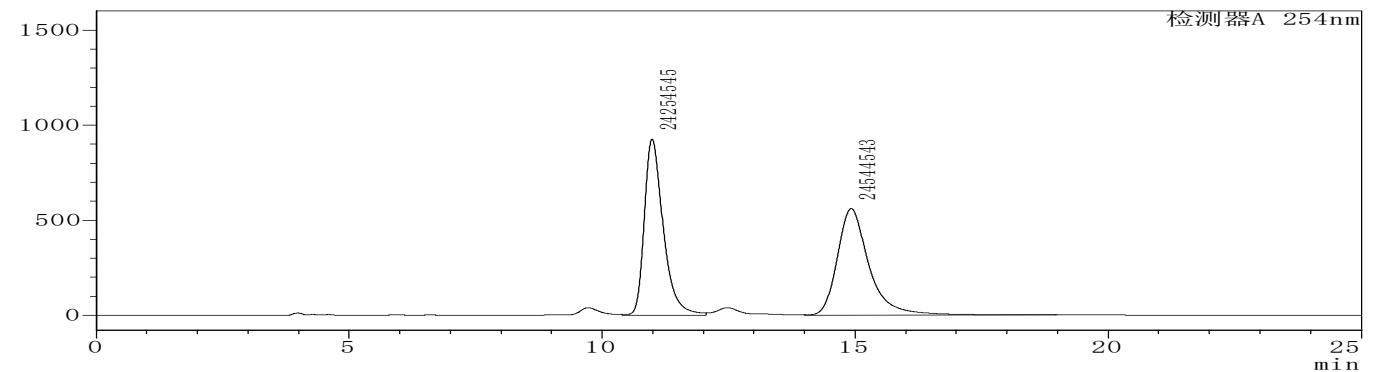

racemic

\begin{tabular}{|c|c|c|c|}
\hline & Retention Time & Area & \%Area \\
\hline 1 & 10.988 & 24254545 & 49.703 \\
\hline 2 & 14.923 & 24544543 & 50.297 \\
\hline
\end{tabular}

$\mathrm{mV}$

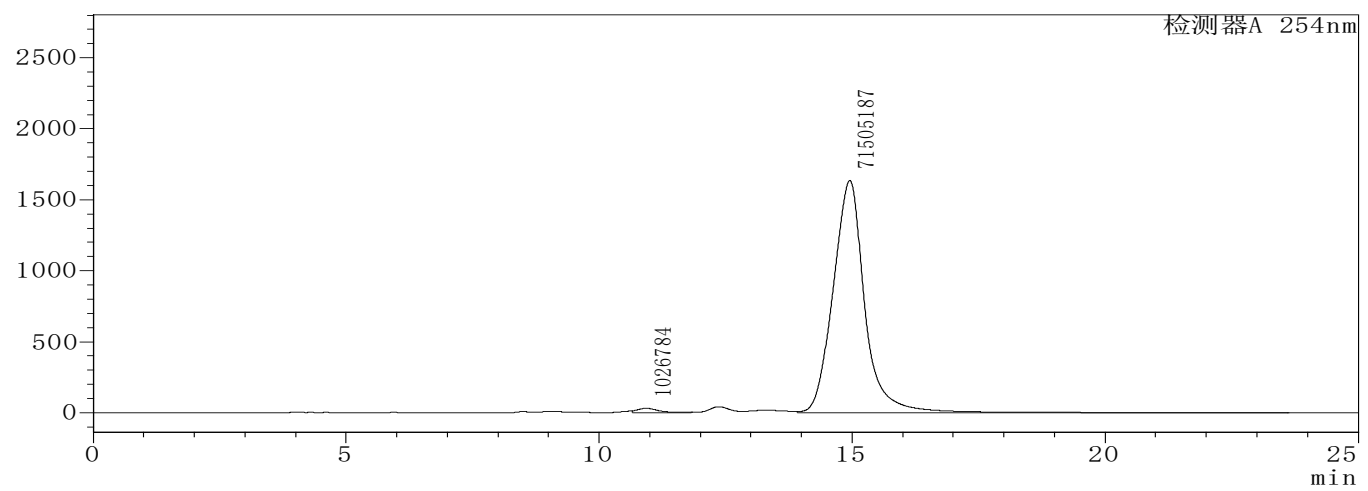

enantio-enriched

\begin{tabular}{|c|c|c|c|}
\hline & Retention Time & Area & \%Area \\
\hline 1 & 10.935 & 1026784 & 1.416 \\
\hline 2 & 14.956 & 71505187 & 98.584 \\
\hline
\end{tabular}


tert-Butyl (S,E)-(1-(2-(4-chlorostyryl)-4-oxo-4H-chromen-3-yl)-3-phenylprop-2yn-1-yl)carbamate (4e)<smiles>CC(C)(C)OC(=O)C#Cc1c(/C=C/c2ccc(Cl)cc2)oc2ccccc2c1=O</smiles>

Compound 4e: Prepared in $0.2 \mathrm{mmol}$ scale using 1.2 equiv of $\mathbf{1 a}$ at $70{ }^{\circ} \mathrm{C}$ for $48 \mathrm{~h} .86 \%$ yield $(88.0 \mathrm{mg})$, yellow solid; mp: 97.9-99.5 ${ }^{\circ} \mathrm{C}, 96 \%$ ee. HPLC (chiral ID column), hexane $/ i-\mathrm{PrOH}=70 / 30$, flow rate $0.8 \mathrm{ml} / \mathrm{min}, \lambda=254 \mathrm{~nm}$, $\operatorname{tr}($ minor $)=9.22 \mathrm{~min}, \mathrm{tr}($ major $)=11.66 \mathrm{~min}$. $[\alpha]^{25} \mathrm{D}=-164.9\left(\mathrm{c}=1.0\right.$ in $\left.\mathrm{CH}_{2} \mathrm{Cl}_{2}\right)$.

${ }^{1} \mathrm{H}$ NMR $\left(400 \mathrm{MHz}, \mathrm{CDCl}_{3}\right) \delta 8.22(\mathrm{~d}, J=8.0 \mathrm{~Hz}, 1 \mathrm{H})$, $7.73-7.66(\mathrm{~m}, 2 \mathrm{H}), 7.61(\mathrm{~d}, J=8.2 \mathrm{~Hz}, 2 \mathrm{H}), 7.53(\mathrm{~d}, J=8.4 \mathrm{~Hz}, 1 \mathrm{H}), 7.48-7.37(\mathrm{~m}$, $6 \mathrm{H}), 7.29-7.20(\mathrm{~m}, 3 \mathrm{H}), 6.64(\mathrm{~d}, J=7.4 \mathrm{~Hz}, 1 \mathrm{H}), 6.18(\mathrm{~d}, J=9.2 \mathrm{~Hz}, 1 \mathrm{H}), 1.45$ (s, 9H). ${ }^{13} \mathrm{C}\{1 \mathrm{H}\} \mathrm{NMR}\left(150 \mathrm{MHz}, \mathrm{CDCl}_{3}\right) \delta 157.9,155.5,155.2,137.8,136.1,134.2$, $133.8,132.0,129.4,129.4,128.4,128.2,126.0,125.2$, 123.7, 122.8, 119.0, 117.8, 117.4, 87.2, 82.6, 80.2, 40.2, 28.5. HRMS (FTMS-ESI), $\mathrm{m} / \mathrm{z}$ calcd for $\mathrm{C}_{31} \mathrm{H}_{26} \mathrm{ClNNaO}_{4}{ }^{+}\left([\mathrm{M}+\mathrm{Na}]^{+}\right) 534.1443\left(535.1476\right.$ for $\left.{ }^{37} \mathrm{Cl}\right)$, found 534.1453 $\left(535.1486\right.$ for $\left.{ }^{37} \mathrm{Cl}\right)$.

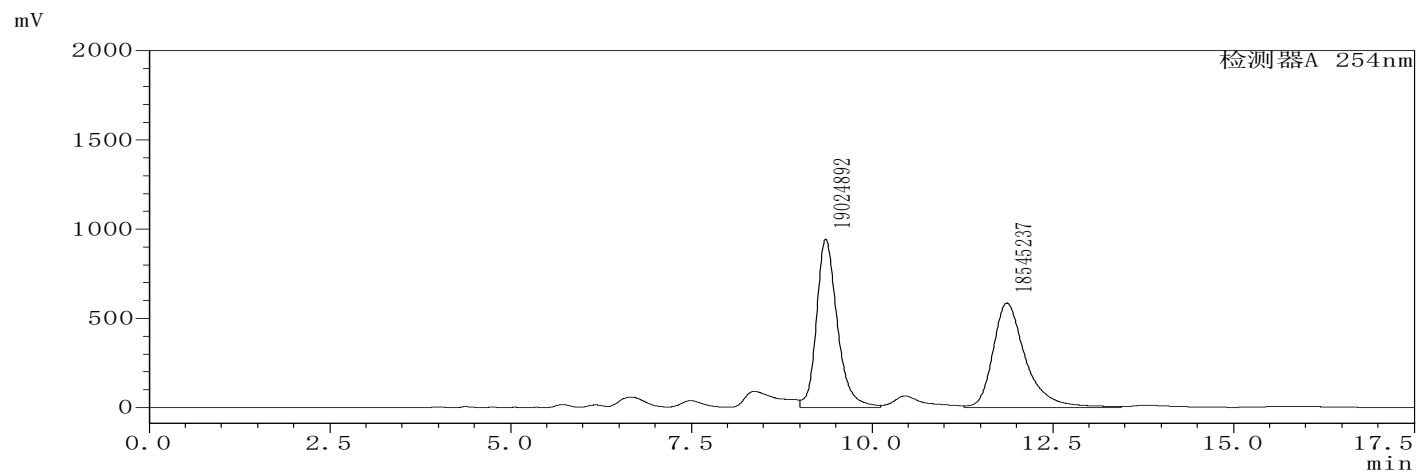

racemic

\begin{tabular}{|c|c|c|c|}
\hline & Retention Time & Area & \%Area \\
\hline 1 & 9.360 & 19024892 & 50.638 \\
\hline 2 & 11.867 & 18545237 & 49.362 \\
\hline
\end{tabular}

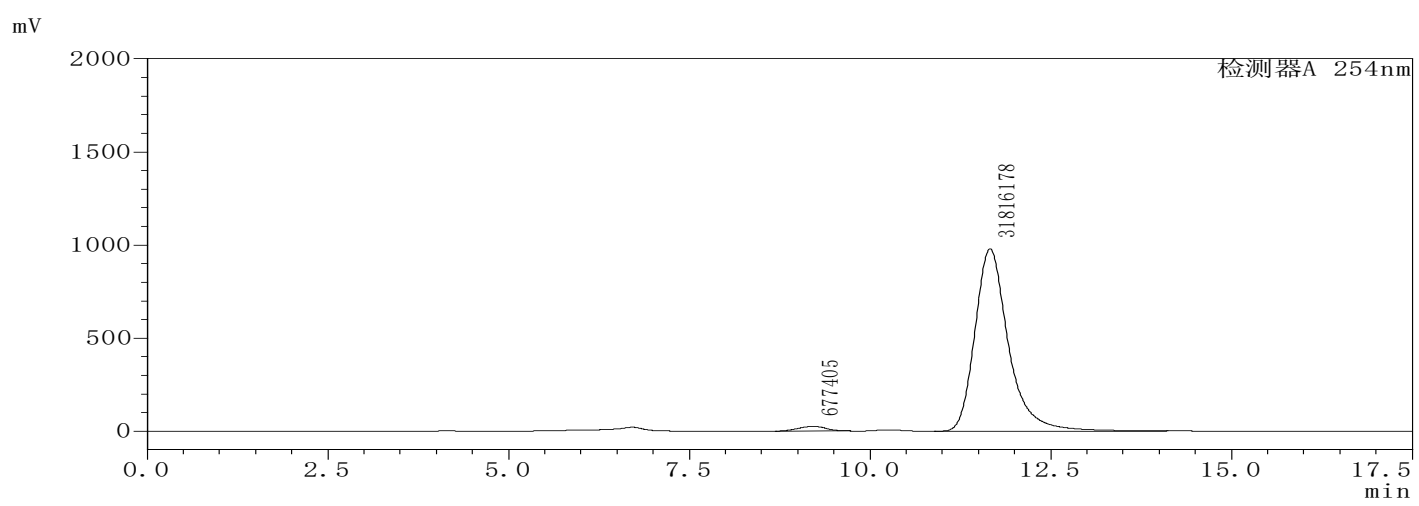

enantio-enriched

\begin{tabular}{|c|c|c|c|}
\hline & Retention Time & Area & \%Area \\
\hline 1 & 9.220 & 677405 & 2.085 \\
\hline 2 & 11.661 & 31816178 & 97.915 \\
\hline
\end{tabular}


tert-Butyl $(S, E)$-(1-(2-(4-bromostyryl)-4-oxo-4H-chromen-3-yl)-3-phenylprop-2yn-1-yl)carbamate (4f)<smiles>CC(C)(C)OC(=O)[C@H](C#Cc1ccccc1)c1c(/C=C/c2ccc(Br)cc2)oc2ccccc2c1=O</smiles>

Compound 4f: Prepared in $0.2 \mathrm{mmol}$ scale using 1.2 equiv of $1 \mathrm{a}$ at $70{ }^{\circ} \mathrm{C}$ for $48 \mathrm{~h} .78 \%$ yield $(86.6 \mathrm{mg})$, yellow solid; mp: 103.2-105.6 ${ }^{\circ} \mathrm{C}, 94 \%$ ee. HPLC (chiral ID column), hexane $/ i-\mathrm{PrOH}=70 / 30$, flow rate $0.8 \mathrm{ml} / \mathrm{min}, \lambda=254 \mathrm{~nm}$, $\operatorname{tr}($ minor $)=11.51 \mathrm{~min}, \mathrm{tr}$ (major) $=15.72 \mathrm{~min}$. $[\alpha]^{25} \mathrm{D}=-207.3\left(\mathrm{c}=1.0\right.$ in $\left.\mathrm{CH}_{2} \mathrm{Cl}_{2}\right)$.

${ }^{1} \mathrm{H}$ NMR $\left(400 \mathrm{MHz}, \mathrm{CDCl}_{3}\right) \delta 8.18(\mathrm{~d}, J=7.8 \mathrm{~Hz}, 1 \mathrm{H})$, $7.72-7.66(\mathrm{~m}, 1 \mathrm{H}), 7.63(\mathrm{~d}, J=15.8 \mathrm{~Hz}, 1 \mathrm{H}), 7.55-7.50(\mathrm{~m}, 4 \mathrm{H}), 7.48(\mathrm{~d}, J=16.2$ $\mathrm{Hz}, 1 \mathrm{H}), 7.43-7.35(\mathrm{~m}, 3 \mathrm{H}), 7.29-7.19(\mathrm{~m}, 4 \mathrm{H}), 6.65(\mathrm{~d}, J=8.2 \mathrm{~Hz}, 1 \mathrm{H}), 6.19$ (d, $J$ $=9.2 \mathrm{~Hz}, 1 \mathrm{H}), 1.45(\mathrm{~s}, 9 \mathrm{H}) .{ }^{13} \mathrm{C}\{1 \mathrm{H}\} \mathrm{NMR}\left(150 \mathrm{MHz}, \mathrm{CDCl}_{3}\right) \delta 177.6,157.8,155.4$, $155.2,137.8,134.2,134.1,132.3,132.0,129.5,128.4,128.2,125.9,125.1,124.4$, 123.6, 122.8, 118.9, 117.7, 117.4, 87.2, 82.6, 80.2, 40.2, 28.5. HRMS (FTMS-ESI), $\mathrm{m} / \mathrm{z}$ calcd for $\mathrm{C}_{31} \mathrm{H}_{26} \mathrm{BrNNaO}_{4}{ }^{+}\left([\mathrm{M}+\mathrm{Na}]^{+}\right) 578.0937$ (580.0917 for ${ }^{81} \mathrm{Br}$ ), found 578.0945 (580.0927 for $\left.{ }^{81} \mathrm{Br}\right)$.

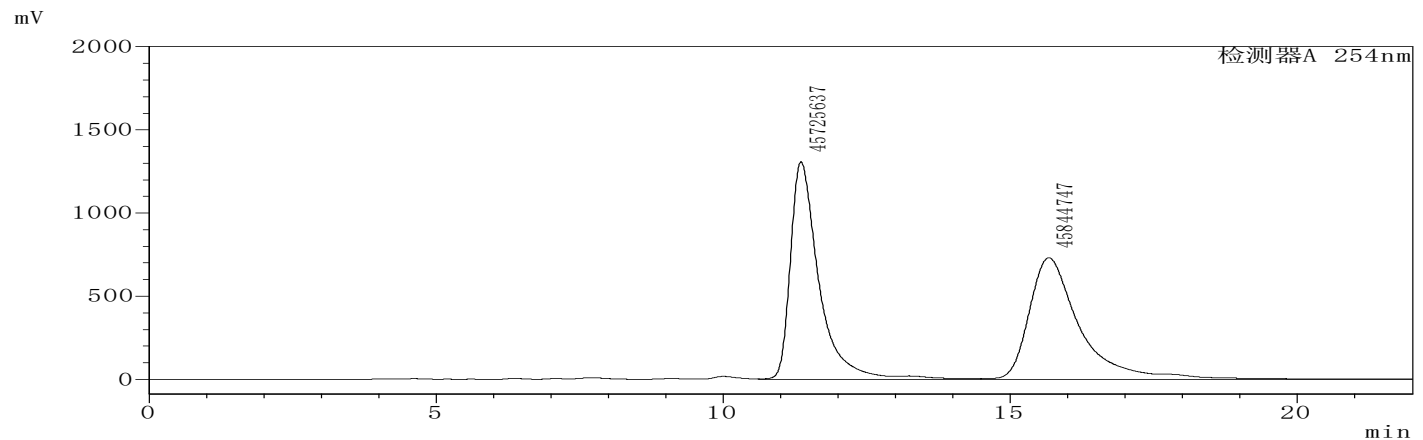

racemic

\begin{tabular}{|c|c|c|c|}
\hline & Retention Time & Area & \%Area \\
\hline 1 & 11.355 & 45725637 & 49.935 \\
\hline 2 & 15.669 & 45844747 & 50.065 \\
\hline
\end{tabular}

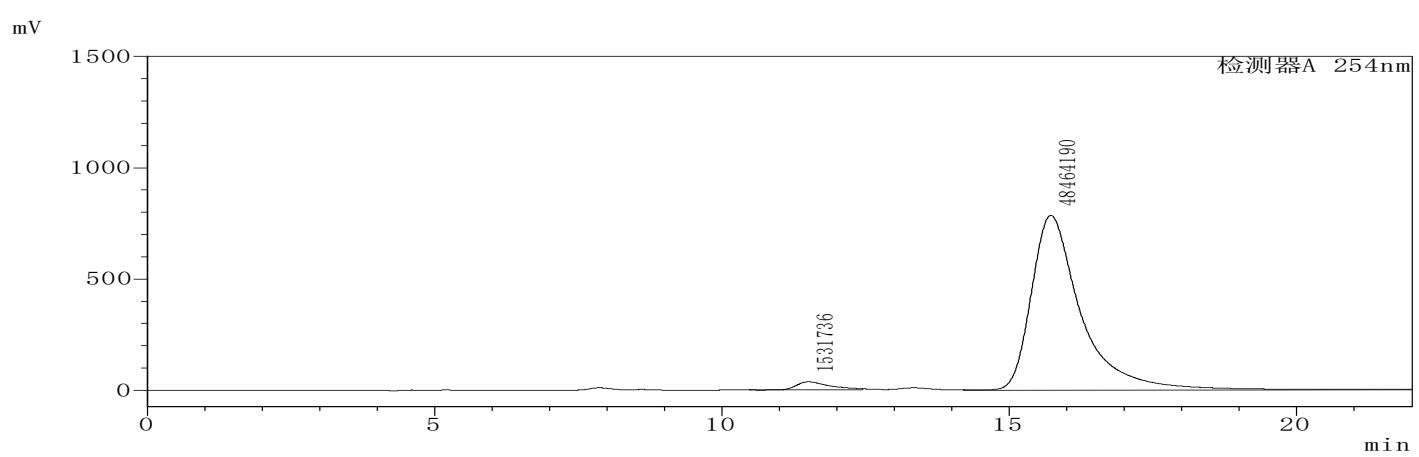

enantio-enriched

\begin{tabular}{|c|c|c|c|}
\hline & Retention Time & Area & \%Area \\
\hline 1 & 11.510 & 1531736 & 3.064 \\
\hline 2 & 15.722 & 48464190 & 96.936 \\
\hline
\end{tabular}


tert-Butyl $(S, E)-(1-(6-m e t h y l-4-o x o-2-s t y r y l-4 H$-chromen-3-yl)-3-phenylprop-2-

yn-1-yl)carbamate (4g)<smiles>Cc1ccc2oc(/C=C/c3ccccc3)c([C@H](C#Cc3ccccc3)N[R6](=O)[O-])c(=O)c2c1</smiles>

Compound 4g: Prepared in $0.2 \mathrm{mmol}$ scale using 1.2 equiv of $1 \mathrm{a}$ at $70{ }^{\circ} \mathrm{C}$ for $66 \mathrm{~h} .93 \%$ yield $(91.0 \mathrm{mg})$, yellow solid; mp: $134.6-136.1{ }^{\circ} \mathrm{C}$, 98\% ee. HPLC (chiral ID column), hexane $/ i-\mathrm{PrOH}=80 / 20$, flow rate $0.8 \mathrm{ml} / \mathrm{min}, \lambda$ $=254 \mathrm{~nm}, \operatorname{tr}($ minor $)=14.89 \mathrm{~min}, t \mathrm{r}$ (major) $=17.53 \mathrm{~min}$. $[\alpha]^{25} \mathrm{D}=-143.3\left(\mathrm{c}=1.0\right.$ in $\left.\mathrm{CH}_{2} \mathrm{Cl}_{2}\right)$.

${ }^{1} \mathrm{H}$ NMR $\left(400 \mathrm{MHz}, \mathrm{CDCl}_{3}\right) \delta 7.98(\mathrm{~s}, 1 \mathrm{H}), 7.71(\mathrm{~d}, J=15.8 \mathrm{~Hz}, 1 \mathrm{H}), 7.66(\mathrm{~d}, J=6.8$ $\mathrm{Hz}, 2 \mathrm{H}), 7.50(\mathrm{dd}, J=8.6,1.8 \mathrm{~Hz}, 1 \mathrm{H}), 7.46-7.38(\mathrm{~m}, 7 \mathrm{H}), 7.26-7.23(\mathrm{~m}, 3 \mathrm{H})$, $6.70(\mathrm{~d}, J=8.4 \mathrm{~Hz}, 1 \mathrm{H}), 6.20(\mathrm{~d}, J=9.4 \mathrm{~Hz}, 1 \mathrm{H}), 2.45(\mathrm{~s}, 3 \mathrm{H}), 1.45(\mathrm{~s}, 9 \mathrm{H}) .{ }^{13} \mathrm{C}\{1 \mathrm{H}\}$ NMR $\left(150 \mathrm{MHz}, \mathrm{CDCl}_{3}\right) \delta 177.8,158.1,155.2,153.8,139.1,135.3,135.3,135.0$, 132.0, 130.1, 129.1, 128.3, 128.2, 128.2, 125.2, 123.3, 122.9, 118.5, 117. 6, 116.9, 87.5, 82.4, 80.0, 40.2, 28.5, 21.1. HRMS (FTMS-ESI), m/z calcd for $\mathrm{C}_{32} \mathrm{H}_{29} \mathrm{NNaO}_{4}{ }^{+}$ $\left([\mathrm{M}+\mathrm{Na}]^{+}\right)$514.1989, found 514.1990.

$\mathrm{mV}$

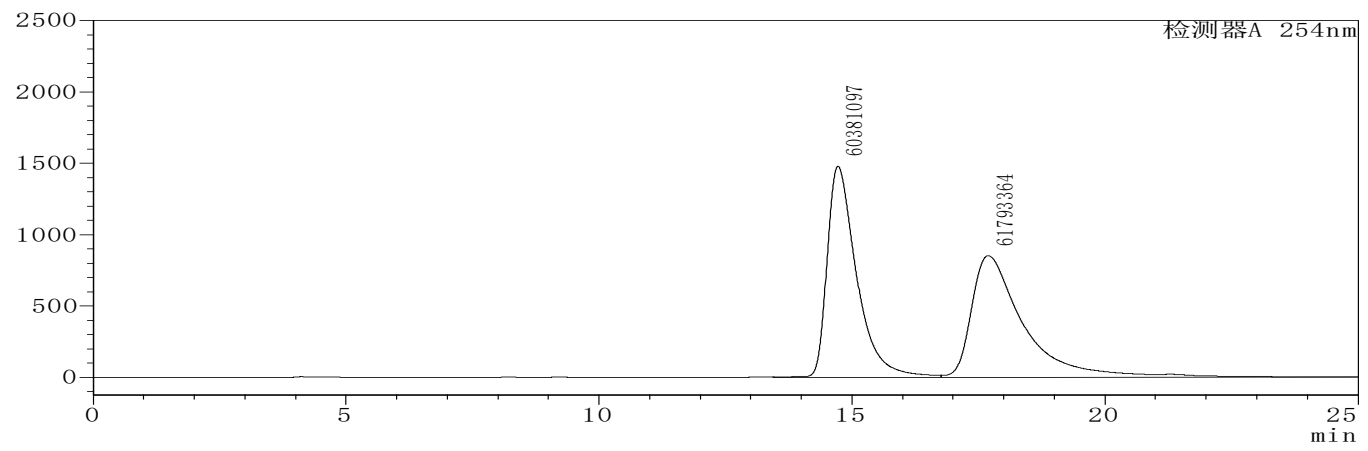

racemic

\begin{tabular}{|c|c|c|c|}
\hline & Retention Time & Area & \%Area \\
\hline 1 & 14.722 & 60381097 & 49.422 \\
\hline 2 & 17.696 & 61793364 & 50.578 \\
\hline
\end{tabular}

$\mathrm{mV}$

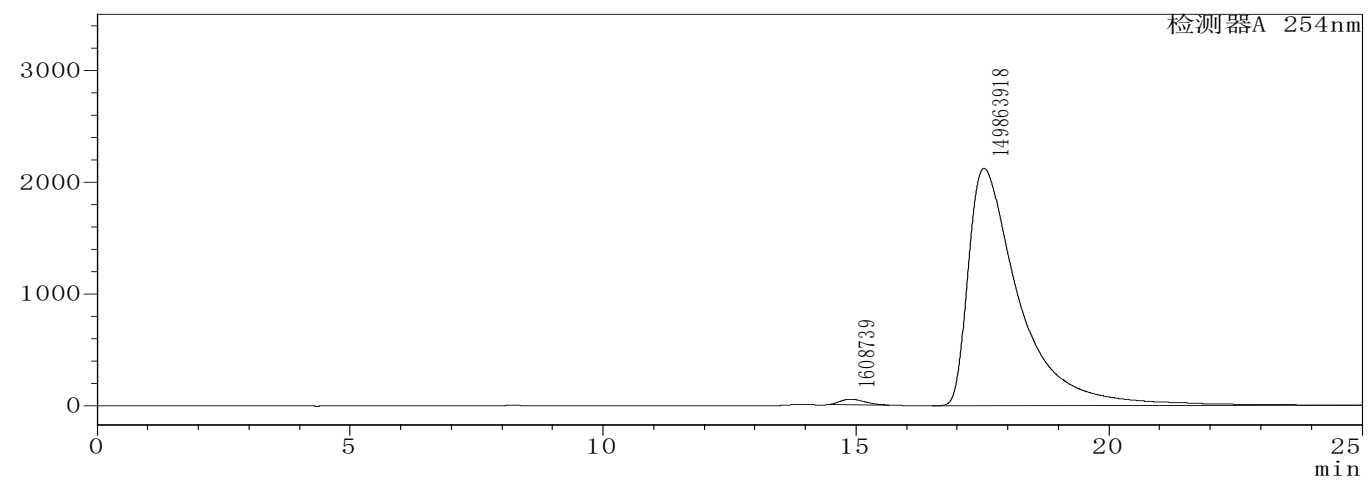

enantio-enriched

\begin{tabular}{|c|c|c|c|}
\hline & Retention Time & Area & \%Area \\
\hline 1 & 14.893 & 1608739 & 1.062 \\
\hline 2 & 17.531 & 149863918 & 98.938 \\
\hline
\end{tabular}


tert-Butyl $(S, E)-(1-(6-c h l o r o-4-0 x o-2-s t y r y l-4 H$-chromen-3-yl)-3-phenylprop-2yn-1-yl)carbamate (4h)<smiles>CC(C)(C)OC(=O)N[C@@H](C#Cc1c(/C=C/c2ccccc2)oc2ccc(Cl)cc2c1=O)C(=O)OC(C)(C)C</smiles>

Compound 4h: Prepared in $0.2 \mathrm{mmol}$ scale using 1.2 equiv of $1 \mathrm{a}$ at $70{ }^{\circ} \mathrm{C}$ for $72 \mathrm{~h} .93 \%$ yield $(95.6 \mathrm{mg}$ ), yellow solid; mp: 83.7-85.4 ${ }^{\circ} \mathrm{C}, 94 \%$ ee. HPLC (chiral ID column), hexane $/ i-\mathrm{PrOH}=70 / 30$, flow rate 0.8 $\mathrm{ml} / \mathrm{min}, \lambda=254 \mathrm{~nm}, t \mathrm{r}($ minor $)=11.85 \mathrm{~min}, \operatorname{tr}($ major $)=$ $15.61 \mathrm{~min}$.

$[\alpha]^{25}{ }_{D}=-125.5\left(\mathrm{c}=1.0\right.$ in $\left.\mathrm{CH}_{2} \mathrm{Cl}_{2}\right)$.

${ }^{1} \mathrm{H}$ NMR $\left(400 \mathrm{MHz}, \mathrm{CDCl}_{3}\right) \delta 8.12(\mathrm{~d}, J=2.2 \mathrm{~Hz}, 1 \mathrm{H}), 7.69(\mathrm{~d}, J=15.8 \mathrm{~Hz}, 1 \mathrm{H})$, $7.65-7.59(\mathrm{~m}, 3 \mathrm{H}), 7.49(\mathrm{~d}, J=9.0 \mathrm{~Hz}, 1 \mathrm{H}), 7.45-7.35(\mathrm{~m}, 6 \mathrm{H}), 7.30-7.21(\mathrm{~m}$, $3 \mathrm{H}), 6.59(\mathrm{~d}, J=8.6 \mathrm{~Hz}, 1 \mathrm{H}), 6.20(\mathrm{~d}, J=9.2 \mathrm{~Hz}, 1 \mathrm{H}), 1.46(\mathrm{~s}, 9 \mathrm{H}) .{ }^{13} \mathrm{C}\{1 \mathrm{H}\} \mathrm{NMR}$ $\left(150 \mathrm{MHz}, \mathrm{CDCl}_{3}\right) \delta 176.5,158.5,155.1,153.8,139.7,135.0,134.2,132.0,131.0$, 130.4, 129.1, 128.5, 128.3, 128.2, 125.3, 124.5, 122.7, 119.5, 118.7, 116.4, 87.0, 82.7, 80.2, 40.2, 28.5. HRMS (FTMS-ESI), m/z calcd for $\mathrm{C}_{31} \mathrm{H}_{26} \mathrm{ClNNaO}_{4}{ }^{+}\left([\mathrm{M}+\mathrm{Na}]^{+}\right)$ $534.1443\left(535.1476\right.$ for $\left.{ }^{37} \mathrm{Cl}\right)$, found 534.1445 (535.1476 for $\left.{ }^{37} \mathrm{Cl}\right)$.

$\mathrm{mV}$

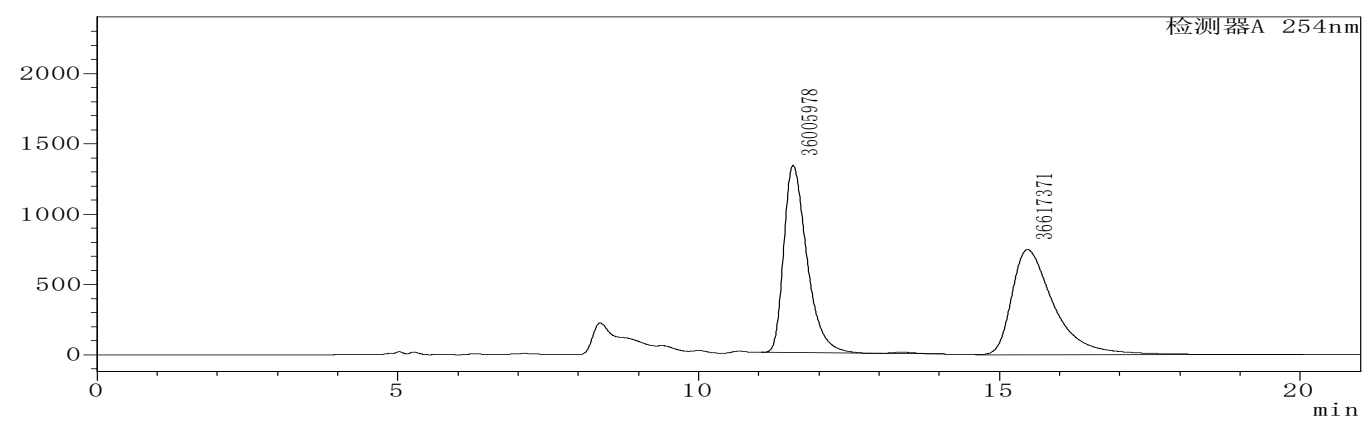

racemic

\begin{tabular}{|c|c|c|c|}
\hline & Retention Time & Area & \%Area \\
\hline 1 & 11.572 & 36005978 & 49.579 \\
\hline 2 & 15.471 & 36617371 & 50.421 \\
\hline
\end{tabular}

$\mathrm{mV}$

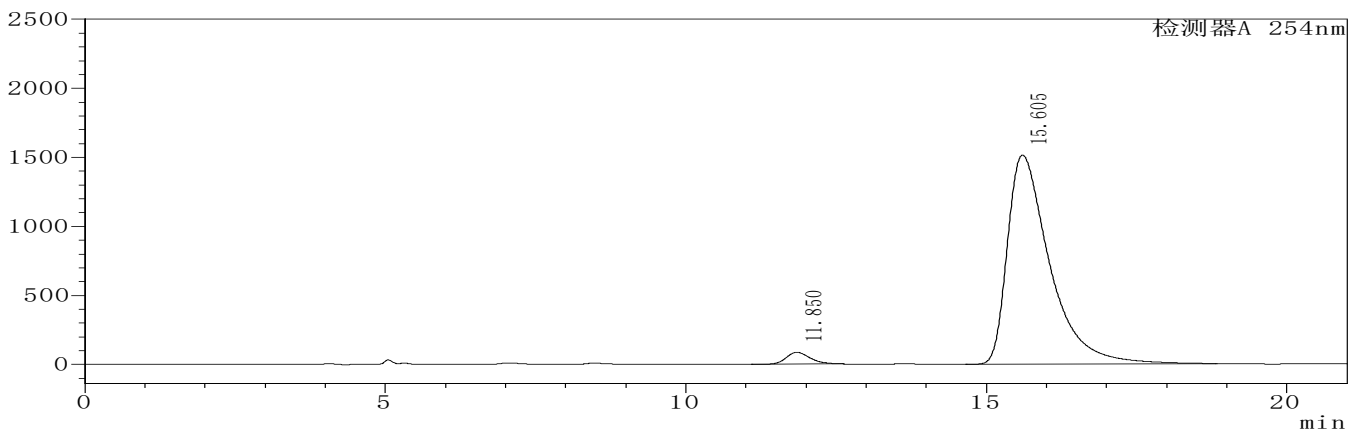

enantio-enriched

\begin{tabular}{|c|c|c|c|}
\hline & Retention Time & Area & \%Area \\
\hline 1 & 11.850 & 2373871 & 3.099 \\
\hline 2 & 15.605 & 74230753 & 96.901 \\
\hline
\end{tabular}


tert-Butyl $(S, E)-(1-(6-b r o m o-4-0 x o-2-s t y r y l-4 H$-chromen-3-yl)-3-phenylprop-2yn-1-yl)carbamate (4i)<smiles>CC(C)(C)OC(=O)[C@H](C#Cc1ccccc1)c1c(/C=C/c2ccccc2)oc2ccc(Br)cc2c1=O</smiles>

Compound 4i: Prepared in $0.2 \mathrm{mmol}$ scale using 1.2 equiv of $1 \mathrm{a}$ at $70{ }^{\circ} \mathrm{C}$ for $72 \mathrm{~h} .80 \%$ yield $(88.5 \mathrm{mg})$, yellow solid; mp: $186.2-187.6^{\circ} \mathrm{C}, 90 \%$ ee. HPLC (chiral ID column), hexane $/ i-\mathrm{PrOH}=70 / 30$, flow rate 0.8 $\mathrm{ml} / \mathrm{min}, \lambda=254 \mathrm{~nm}, t \mathrm{r}($ minor $)=11.31 \mathrm{~min}, \operatorname{tr}($ major $)=$ $14.92 \mathrm{~min}$.

$[\alpha]^{25}{ }_{D}=-164.9\left(\mathrm{c}=1.0\right.$ in $\left.\mathrm{CH}_{2} \mathrm{Cl}_{2}\right)$.

${ }^{1} \mathrm{H}$ NMR $\left(400 \mathrm{MHz}, \mathrm{CDCl}_{3}\right) \delta 8.06(\mathrm{~d}, J=8.6 \mathrm{~Hz}, 1 \mathrm{H}), 7.75(\mathrm{~s}, 1 \mathrm{H}), 7.70(\mathrm{~d}, J=15.8$ $\mathrm{Hz}, 1 \mathrm{H}), 7.66(\mathrm{~d}, J=6.8 \mathrm{~Hz}, 2 \mathrm{H}), 7.53-7.45(\mathrm{~m}, 2 \mathrm{H}), 7.42(\mathrm{~d}, J=5.4 \mathrm{~Hz}, 5 \mathrm{H}), 7.29$ $-7.20(\mathrm{~m}, 3 \mathrm{H}), 6.59(\mathrm{~d}, J=8.2 \mathrm{~Hz}, 1 \mathrm{H}), 6.20(\mathrm{~d}, J=9.2 \mathrm{~Hz}, 1 \mathrm{H}), 1.46(\mathrm{~s}, 9 \mathrm{H})$. ${ }^{13} \mathrm{C}\{1 \mathrm{H}\} \mathrm{NMR}\left(150 \mathrm{MHz}, \mathrm{CDCl}_{3}\right) \delta 176.9,158.3,155.5,155.1,139.6,135.1,132.0$, $130.4,129.1,128.7,128.4,128.3,128.2$, 127.3, 122.7, 122.5, 120.9, 118.9, 116.5, 87.0, 82.6, 80.2, 40.1, 28.5. HRMS (FTMS-ESI), m/z calcd for $\mathrm{C}_{31} \mathrm{H}_{26} \mathrm{BrNNaO}_{4}{ }^{+}([\mathrm{M}$ $\left.+\mathrm{Na}]^{+}\right) 578.0937$ (580.0917 for ${ }^{81} \mathrm{Br}$ ), found 578.0941 (580.0926 for $\left.{ }^{81} \mathrm{Br}\right)$.

$\mathrm{mV}$

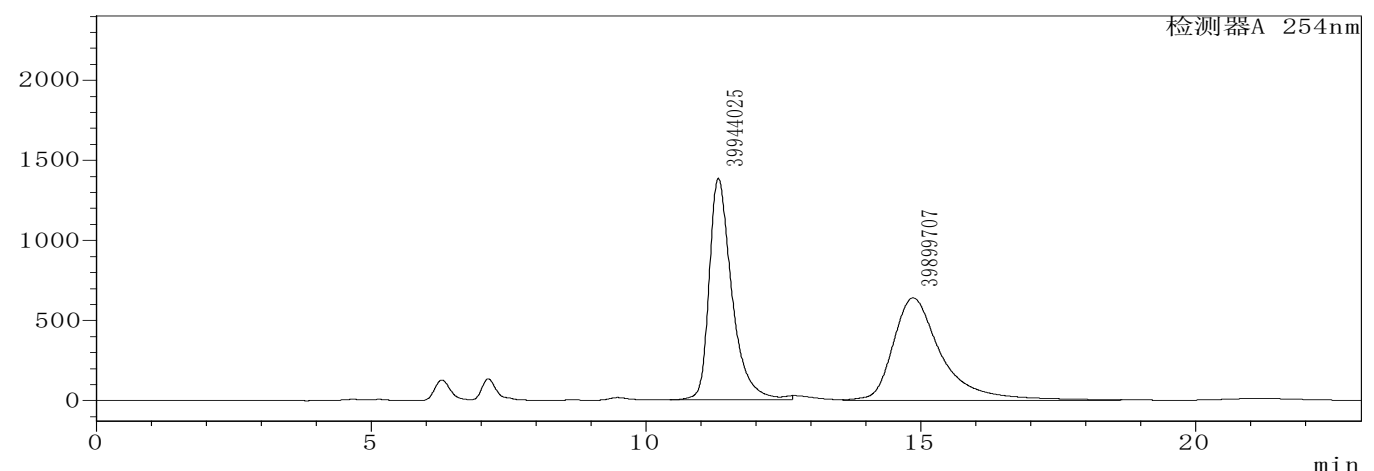

racemic

\begin{tabular}{|c|c|c|c|}
\hline & Retention Time & Area & \%Area \\
\hline 1 & 11.314 & 39944025 & 50.028 \\
\hline 2 & 14.857 & 39899707 & 49.972 \\
\hline
\end{tabular}

$\mathrm{mV}$

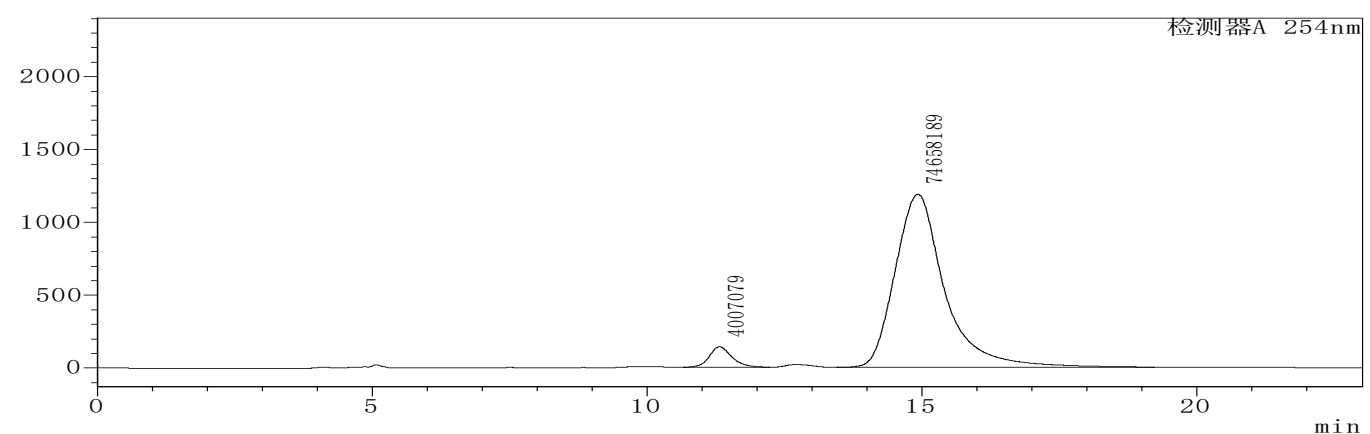

enantio-enriched

\begin{tabular}{|c|c|c|c|}
\hline & Retention Time & Area & \%Area \\
\hline 1 & 11.314 & 4007079 & 5.094 \\
\hline 2 & 14.922 & 74658189 & 94.906 \\
\hline
\end{tabular}


tert-Butyl $(S, E)$-(1-(7-methoxy-4-oxo-2-styryl-4H-chromen-3-yl)-3-phenylprop2-yn-1-yl)carbamate (4j)<smiles>COc1ccc2c(=O)c([C@H](C#Cc3ccccc3)NC(=O)OCc3ccccc3)c(/C=C/c3ccccc3)oc2c1</smiles>

Compound 4j: Prepared in $0.2 \mathrm{mmol}$ scale using 1.2 equiv of $1 \mathrm{a}$ at $70{ }^{\circ} \mathrm{C}$ for $72 \mathrm{~h} .67 \%$ yield $(68.4 \mathrm{mg})$, yellow solid; mp: $76.2-77.4{ }^{\circ} \mathrm{C}, 96 \%$ ee. HPLC (chiral ID column), hexane $/ i$-PrOH $=70 / 30$, flow rate 0.8 $\mathrm{ml} / \mathrm{min}, \lambda=254 \mathrm{~nm}, t \mathrm{r}$ (minor) $=18.14 \mathrm{~min}, t \mathrm{r}$ (major) $=26.41 \mathrm{~min}$.

$[\alpha]^{25}{ }_{D}=-170.2\left(\mathrm{c}=1.0\right.$ in $\left.\mathrm{CH}_{2} \mathrm{Cl}_{2}\right)$.

${ }^{1} \mathrm{H}$ NMR $\left(400 \mathrm{MHz}, \mathrm{CDCl}_{3}\right) \delta 8.08(\mathrm{~d}, J=8.8 \mathrm{~Hz}, 1 \mathrm{H}), 7.70(\mathrm{~d}, J=15.8 \mathrm{~Hz}, 1 \mathrm{H})$, $7.64(\mathrm{~d}, J=6.8 \mathrm{~Hz}, 2 \mathrm{H}), 7.47-7.37(\mathrm{~m}, 6 \mathrm{H}), 7.25(\mathrm{dd}, J=5.6,4.2 \mathrm{~Hz}, 3 \mathrm{H}), 6.97-$ $6.89(\mathrm{~m}, 2 \mathrm{H}), 6.73(\mathrm{~d}, J=8.6 \mathrm{~Hz}, 1 \mathrm{H}), 6.19(\mathrm{~d}, J=9.2 \mathrm{~Hz}, 1 \mathrm{H}), 3.93$ (s, 3H), 1.45 (s, 9H). ${ }^{13} \mathrm{C}\{1 \mathrm{H}\}$ NMR $\left(150 \mathrm{MHz}, \mathrm{CDCl}_{3}\right) \delta 177.1,164.6,157.8,157.3,155.2,138.7$, 135.4, 132.0, 130.1, 129.1, 128.6, 128.3, 128.2, 128.1, 127.3, 122.9, 117.6, 116.9, 114.6, 100.0, 87.5, 82.4, 80.0, 56.0, 40.2, 28.5. HRMS (FTMS-ESI), m/z calcd for $\mathrm{C}_{32} \mathrm{H}_{29} \mathrm{NNaO}_{5}^{+}\left([\mathrm{M}+\mathrm{Na}]^{+}\right) 530.1938$, found 530.1940.

$\mathrm{mV}$

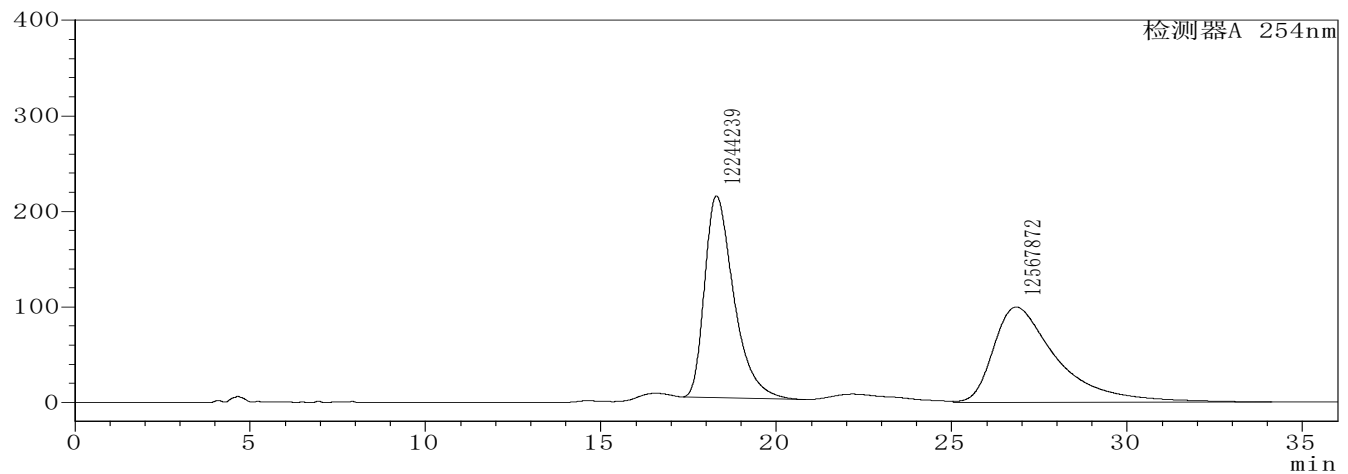

racemic

\begin{tabular}{|c|c|c|c|}
\hline & Retention Time & Area & \%Area \\
\hline 1 & 18.293 & 12244239 & 49.348 \\
\hline 2 & 26.838 & 12567872 & 50.652 \\
\hline
\end{tabular}

$\mathrm{mV}$

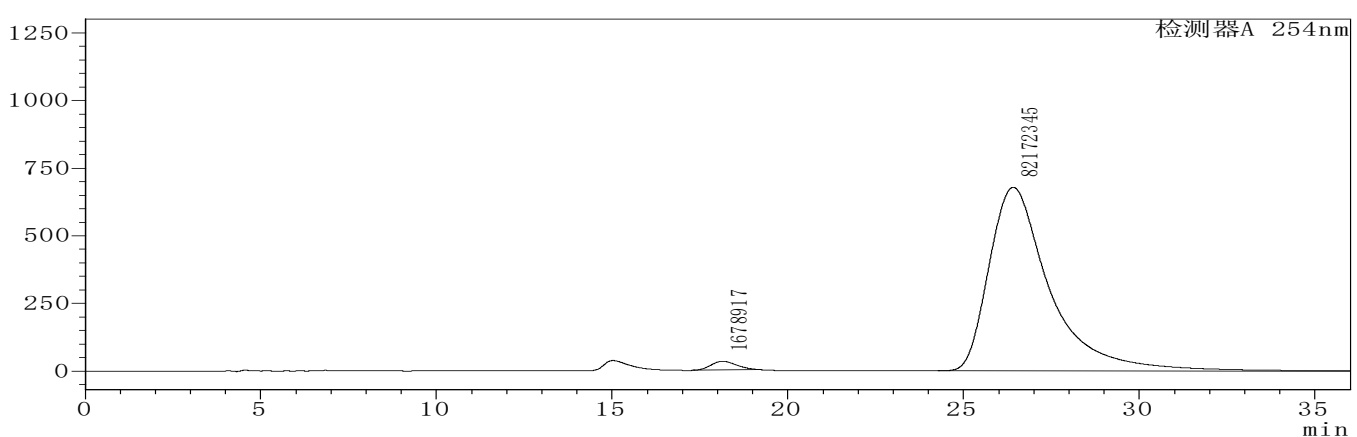

enantio-enriched

\begin{tabular}{|c|c|c|c|}
\hline & Retention Time & Area & \%Area \\
\hline 1 & 18.142 & 1678917 & 2.002 \\
\hline 2 & 26.412 & 82172345 & 97.998 \\
\hline
\end{tabular}


tert-Butyl $(S, E)-(3-(4-f l u o r o p h e n y l)-1-(4-0 x 0-2-s t y r y l-4 H$-chromen-3-yl)prop-2yn-1-yl)carbamate (4k)<smiles>O=C(O)[C@H](C#Cc1ccc(F)cc1)c1c(/C=C/c2ccccc2)oc2ccccc2c1=O</smiles>

Compound 4k: Prepared in $0.2 \mathrm{mmol}$ scale using 1.2 equiv of $\mathbf{1 a}$ at $70{ }^{\circ} \mathrm{C}$ for $48 \mathrm{~h} .62 \%$ yield $(61.5 \mathrm{mg})$, yellow solid; mp: $150.9-152.2{ }^{\circ} \mathrm{C}, 96 \%$ ee. HPLC (chiral ID column), hexane $/ i-\mathrm{PrOH}=70 / 30$, flow rate $0.8 \mathrm{ml} / \mathrm{min}, \lambda=254 \mathrm{~nm}$, $t \mathrm{r}$ (minor) $=9.82 \mathrm{~min}, t \mathrm{r}$ $($ major $)=13.06 \mathrm{~min}$.

$[\alpha]^{25} \mathrm{D}=-148.2\left(\mathrm{c}=1.0\right.$ in $\left.\mathrm{CH}_{2} \mathrm{Cl}_{2}\right)$.

${ }^{1} \mathrm{H}$ NMR $\left(400 \mathrm{MHz}, \mathrm{CDCl}_{3}\right) \delta 8.22(\mathrm{~d}, J=7.8 \mathrm{~Hz}, 1 \mathrm{H}), 7.75(\mathrm{~d}, J=15.8 \mathrm{~Hz}, 1 \mathrm{H})$, $7.71-7.66(\mathrm{~m}, 3 \mathrm{H}), 7.54(\mathrm{~d}, J=8.4 \mathrm{~Hz}, 1 \mathrm{H}), 7.48-7.35(\mathrm{~m}, 7 \mathrm{H}), 6.94(\mathrm{t}, J=8.6 \mathrm{~Hz}$, $2 \mathrm{H}), 6.65(\mathrm{~d}, J=8.2 \mathrm{~Hz}, 1 \mathrm{H}), 6.19(\mathrm{~d}, J=9.4 \mathrm{~Hz}, 1 \mathrm{H}), 1.45(\mathrm{~s}, 9 \mathrm{H}) .{ }^{13} \mathrm{C}\{1 \mathrm{H}\} \mathrm{NMR}$ $\left(150 \mathrm{MHz}, \mathrm{CDCl}_{3}\right) \delta 177.8,162.6(\mathrm{~d}, J=249.0 \mathrm{~Hz}), 158.27,155.6,155.2,139.4$, 135.3, 134.1, 134.0 (d, $J=8.4 \mathrm{~Hz}), 130.3,129.2,128.2,125.9,125.1,123.7,119.0$, 118.6, 117.8, 116.8, 115.49 (d, $J=22.0 \mathrm{~Hz}), 87.1,81.4,80.2,40.2$, 28.5. HRMS (FTMS-ESI), $\mathrm{m} / \mathrm{z}$ calcd for $\mathrm{C}_{31} \mathrm{H}_{26} \mathrm{FNNaO}_{4}{ }^{+}\left([\mathrm{M}+\mathrm{Na}]^{+}\right) 518.1738$, found 518.1744. $\mathrm{mV}$

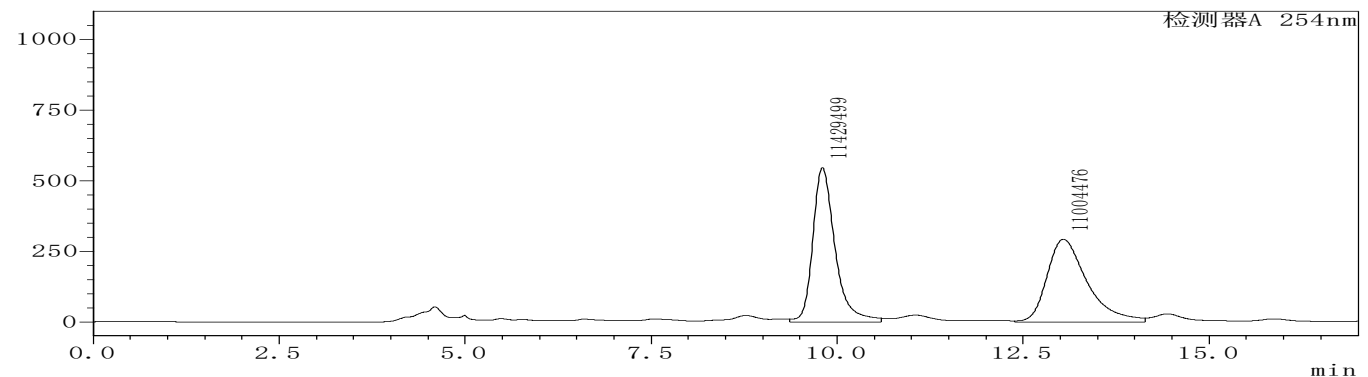

racemic

\begin{tabular}{|c|c|c|c|}
\hline & Retention Time & Area & \%Area \\
\hline 1 & 9.801 & 11429499 & 50.947 \\
\hline 2 & 13.040 & 11004476 & 49.053 \\
\hline
\end{tabular}

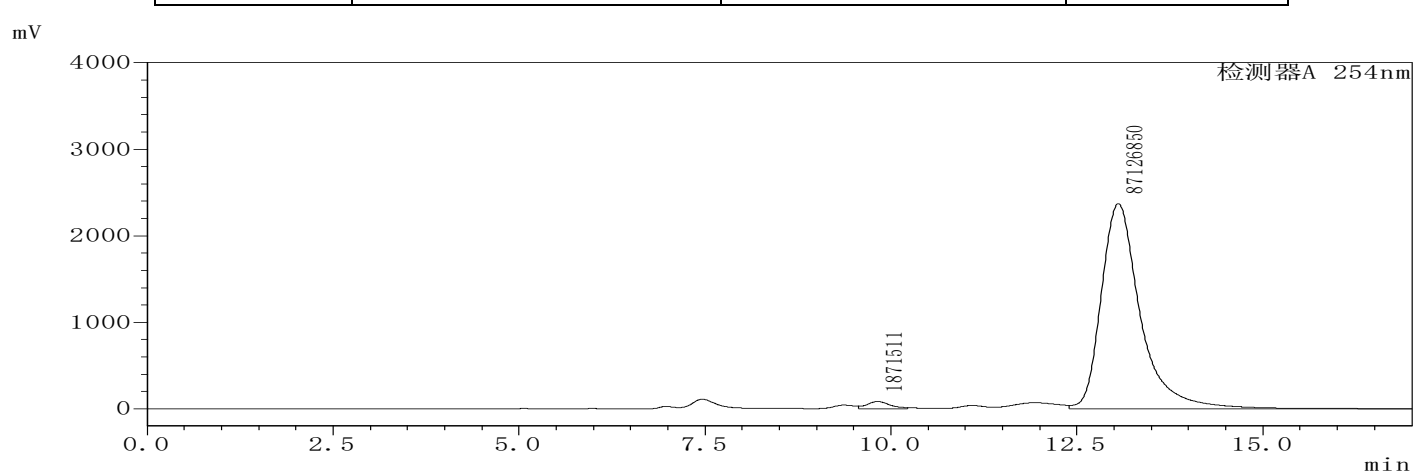

enantio-enriched

\begin{tabular}{|c|c|c|c|}
\hline & Retention Time & Area & \%Area \\
\hline 1 & 9.819 & 1871511 & 2.103 \\
\hline 2 & 13.055 & 87126850 & 97.897 \\
\hline
\end{tabular}


tert-Butyl (S,E)-(3-(4-chlorophenyl)-1-(4-oxo-2-styryl-4H-chromen-3-yl)prop-2yn-1-yl)carbamate (4I)<smiles>O=C([NH2+][C@H](C#Cc1ccccc1)c1c(/C=C/c2ccccc2)oc2ccccc2c1=O)OCc1ccccc1</smiles>

Compound 4l: Prepared in $0.2 \mathrm{mmol}$ scale using 1.2 equiv of $\mathbf{1 a}$ at $70{ }^{\circ} \mathrm{C}$ for $72 \mathrm{~h} .78 \%$ yield $(80.0 \mathrm{mg})$, yellow solid; mp: $64.7-66.0{ }^{\circ} \mathrm{C}$, 90\% ee. HPLC (chiral ID column), hexane $/ i-\mathrm{PrOH}=70 / 30$, flow rate $0.8 \mathrm{ml} / \mathrm{min}, \lambda=254 \mathrm{~nm}, \mathrm{tr}$ (minor) $=11.54 \mathrm{~min}, \mathrm{tr}$ (major) $=15.37 \mathrm{~min}$.

$[\alpha]^{25}{ }_{D}=-195.2\left(\mathrm{c}=1.0\right.$ in $\left.\mathrm{CH}_{2} \mathrm{Cl}_{2}\right)$.

${ }^{1} \mathrm{H}$ NMR $\left(400 \mathrm{MHz}, \mathrm{CDCl}_{3}\right) \delta 8.22(\mathrm{~d}, J=8.0 \mathrm{~Hz}, 1 \mathrm{H}), 7.76(\mathrm{~d}, J=15.8 \mathrm{~Hz}, 1 \mathrm{H})$, $7.71-7.66(\mathrm{~m}, 3 \mathrm{H}), 7.55(\mathrm{~d}, J=8.4 \mathrm{~Hz}, 1 \mathrm{H}), 7.48-7.38(\mathrm{~m}, 5 \mathrm{H}), 7.34(\mathrm{~d}, J=8.4 \mathrm{~Hz}$, 2H), $7.22(\mathrm{~d}, J=7.6 \mathrm{~Hz}, 2 \mathrm{H}), 6.65(\mathrm{~d}, J=8.4 \mathrm{~Hz}, 1 \mathrm{H}), 6.19(\mathrm{~d}, J=9.2 \mathrm{~Hz}, 1 \mathrm{H}), 1.45$ $(\mathrm{s}, 9 \mathrm{H}) .{ }^{13} \mathrm{C}\{1 \mathrm{H}\} \mathrm{NMR}\left(150 \mathrm{MHz}, \mathrm{CDCl}_{3}\right) \delta 177.8,158.3,155.6,155.2,139.5,135.2$, $134.4,134.2$, 133.3, 130.3, 129.2, 128.6, 128.2, 125.9, 125.2, 123.6, 121.4, 118.5, 117.8, 116.7, 88.4, 81.3, 80.2, 40.2, 28.5. HRMS (FTMS-ESI), m/z calcd for $\mathrm{C}_{31} \mathrm{H}_{26} \mathrm{ClNNaO}_{4}{ }^{+}\left([\mathrm{M}+\mathrm{Na}]^{+}\right) 534.1443\left(535.1476\right.$ for $\left.{ }^{37} \mathrm{Cl}\right)$, found 534.1452 $\left(535.1479\right.$ for $\left.{ }^{37} \mathrm{Cl}\right)$.

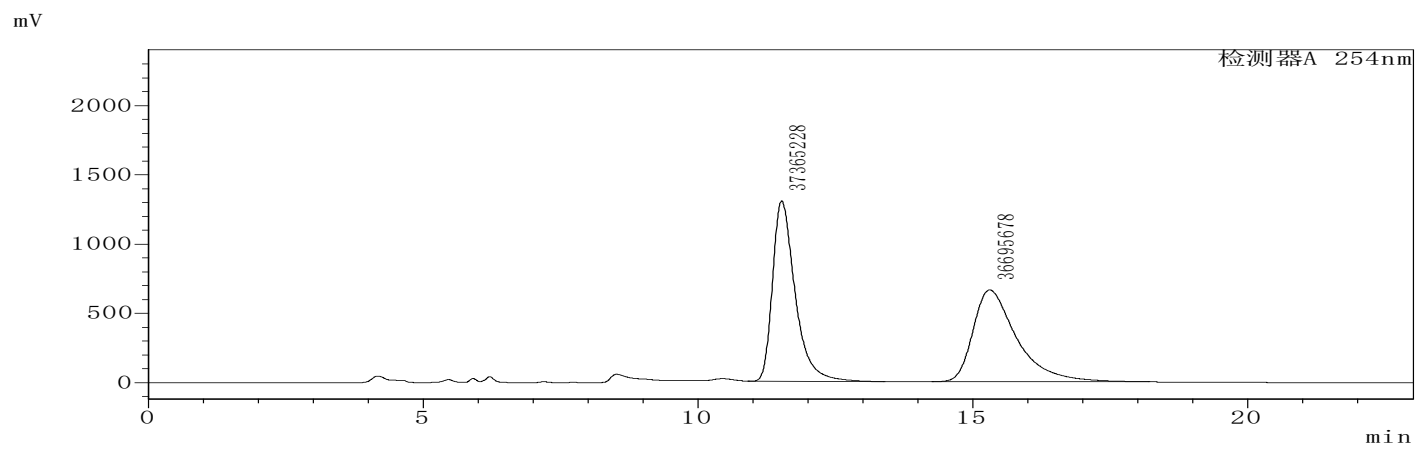

racemic

\begin{tabular}{|c|c|c|c|}
\hline & Retention Time & Area & \%Area \\
\hline 1 & 11.521 & 37365228 & 50.452 \\
\hline 2 & 15.304 & 36695678 & 49.548 \\
\hline
\end{tabular}

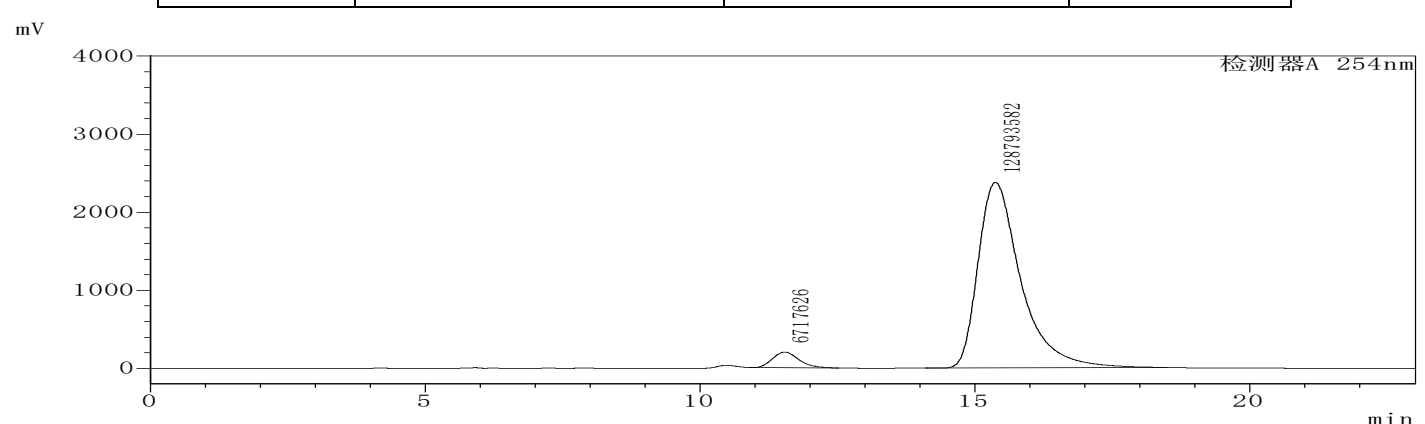

enantio-enriched

\begin{tabular}{|c|c|c|c|}
\hline & Retention Time & Area & \%Area \\
\hline 1 & 11.537 & 6717626 & 4.957 \\
\hline 2 & 15.370 & 128793582 & 95.043 \\
\hline
\end{tabular}


tert-Butyl $(S, E)$-(3-(4-methoxyphenyl)-1-(4-oxo-2-styryl-4H-chromen-3-yl)prop2-yn-1-yl)carbamate (4m)<smiles>COc1ccc(C#C[C@H](NC(=O)OCc2ccccc2)c2c(/C=C/c3ccccc3)oc3ccccc3c2=O)cc1</smiles>

Compound 4m: Prepared in $0.2 \mathrm{mmol}$ scale using 1.2 equiv of $\mathbf{1 a}$ at $70{ }^{\circ} \mathrm{C}$ for 72 h. $90 \%$ yield $(91.2$ $\mathrm{mg}$ ), yellow solid; mp: $73.5-75.1{ }^{\circ} \mathrm{C}, 96 \%$ ee. HPLC (chiral ID column), hexane $/ i-\mathrm{PrOH}=70 / 30$, flow rate $0.8 \mathrm{ml} / \mathrm{min}, \lambda=254 \mathrm{~nm}, t \mathrm{r}$ (minor) $=$ $20.22 \mathrm{~min}, \mathrm{tr}$ (major) $=25.85 \mathrm{~min}$.

$[\alpha]^{25}{ }_{D}=-203.4\left(\mathrm{c}=1.0\right.$ in $\left.\mathrm{CH}_{2} \mathrm{Cl}_{2}\right)$.

${ }^{1} \mathrm{H}$ NMR $\left(400 \mathrm{MHz}, \mathrm{CDCl}_{3}\right) \delta 8.21(\mathrm{~d}, J=8.0 \mathrm{~Hz}, 1 \mathrm{H}), 7.73(\mathrm{~d}, J=16.0 \mathrm{~Hz}, 1 \mathrm{H})$, $7.69-7.65(\mathrm{~m}, 3 \mathrm{H}), 7.55-7.47(\mathrm{~m}, 2 \mathrm{H}), 7.45-7.38(\mathrm{~m}, 4 \mathrm{H}), 7.35(\mathrm{~d}, J=7.4 \mathrm{~Hz}$, 2H), $6.77(\mathrm{~d}, J=7.4 \mathrm{~Hz}, 2 \mathrm{H}), 6.65(\mathrm{~d}, J=7.2 \mathrm{~Hz}, 1 \mathrm{H}), 6.20(\mathrm{~d}, J=9.0 \mathrm{~Hz}, 1 \mathrm{H}), 3.76$ $(\mathrm{s}, 3 \mathrm{H}), 1.45(\mathrm{~s}, 9 \mathrm{H}) .{ }^{13} \mathrm{C}\{1 \mathrm{H}\} \mathrm{NMR}\left(150 \mathrm{MHz}, \mathrm{CDCl}_{3}\right) \delta 177.7,159.7,158.2,155.5$, 155.2 , 139.2, 135.3, 134.1, 133.5, 130.2, 129.1, 128.2, 125.9, 125.0, 123.7, 118.9, 117.8, 116.9, 115.0, 113.8, 85.9, 82.4, 80.0, 55.3, 40.2, 28.5. HRMS (FTMS-ESI), $\mathrm{m} / \mathrm{z}$ calcd for $\mathrm{C}_{32} \mathrm{H}_{29} \mathrm{NNaO}_{5}{ }^{+}\left([\mathrm{M}+\mathrm{Na}]^{+}\right) 530.1938$, found 530.1946.

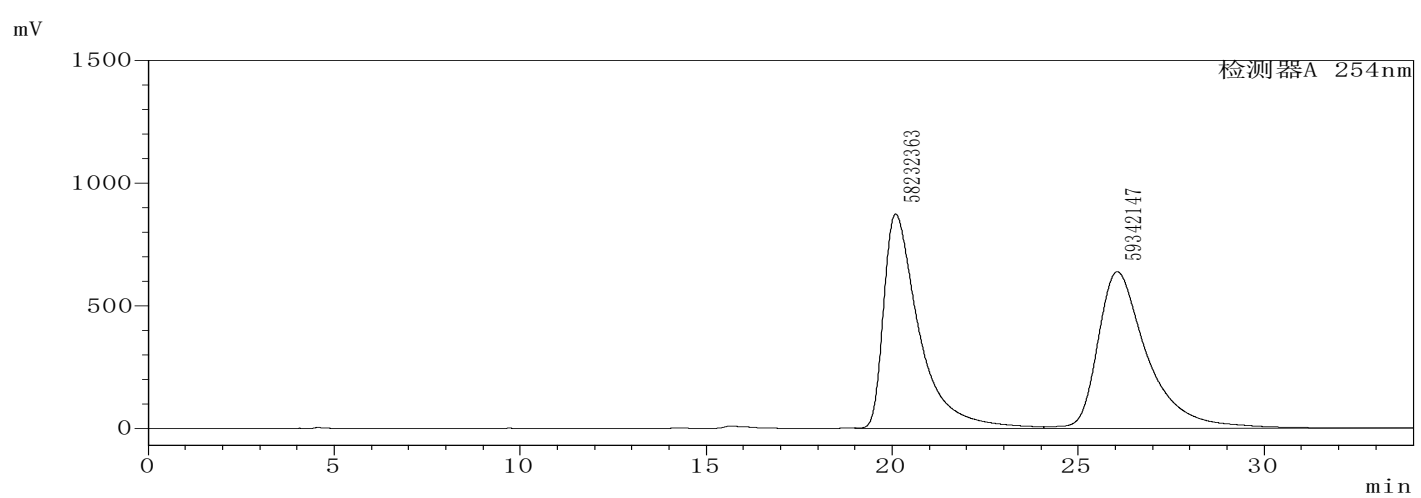

racemic

\begin{tabular}{|c|c|c|c|}
\hline & Retention Time & Area & \%Area \\
\hline 1 & 20.094 & 58232363 & 49.528 \\
\hline 2 & 26.050 & 59342147 & 50.472 \\
\hline
\end{tabular}

$\mathrm{mV}$

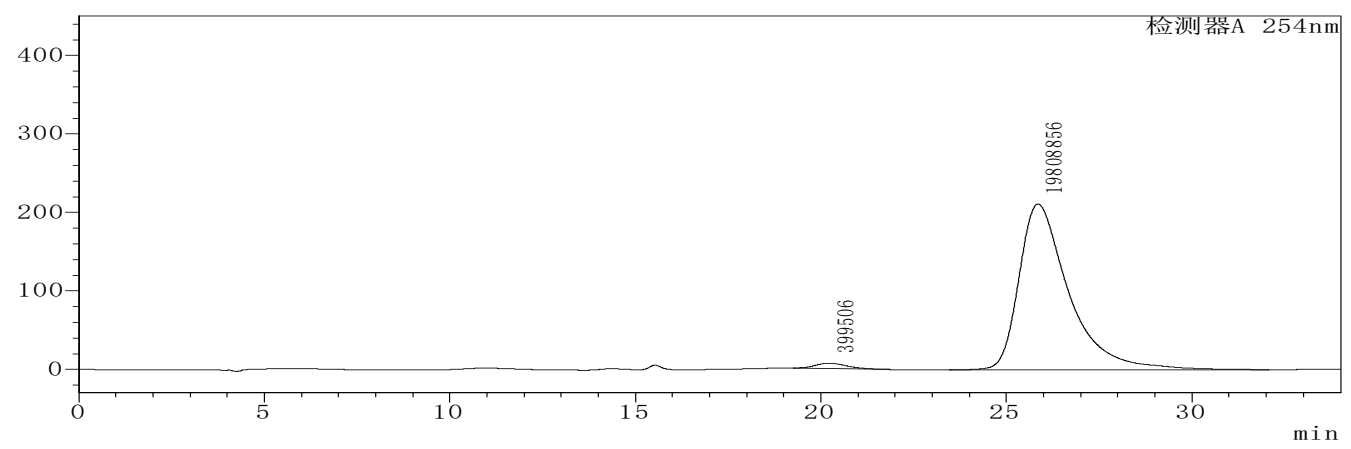

enantio-enriched

\begin{tabular}{|c|c|c|c|}
\hline & Retention Time & Area & \%Area \\
\hline 1 & 20.216 & 399506 & 1.977 \\
\hline 2 & 25.848 & 19808856 & 98.023 \\
\hline
\end{tabular}


tert-Butyl $(S, E)$-(3-(2-methoxyphenyl)-1-(4-oxo-2-styryl-4H-chromen-3-yl)prop2-yn-1-yl)carbamate (4n)<smiles>COc1ccccc1C#C[C@H](NC(=O)OCc1ccccc1)c1c(/C=C/c2ccccc2)oc2ccccc2c1=O</smiles>

Compound 4n: Prepared in $0.2 \mathrm{mmol}$ scale using 1.2 equiv of $1 \mathrm{a}$ at $70{ }^{\circ} \mathrm{C}$ for $70 \mathrm{~h} .87 \%$ yield $(88.0 \mathrm{mg})$, yellow solid; mp: $56.4-58.2{ }^{\circ} \mathrm{C}, 95 \%$ ee. HPLC (chiral ID column), hexane $/ i-\mathrm{PrOH}=70 / 30$, flow rate 0.8 $\mathrm{ml} / \mathrm{min}, \lambda=254 \mathrm{~nm}, t \mathrm{r}($ minor $)=19.16 \mathrm{~min}, t \mathrm{r}($ major $)=$ $31.83 \mathrm{~min}$.

$[\alpha]^{25}{ }_{D}=-101.6\left(\mathrm{c}=1.0\right.$ in $\left.\mathrm{CH}_{2} \mathrm{Cl}_{2}\right)$.

${ }^{1} \mathrm{H}$ NMR $\left(400 \mathrm{MHz}, \mathrm{CDCl}_{3}\right) \delta 8.21(\mathrm{~d}, J=7.8 \mathrm{~Hz}, 1 \mathrm{H}), 7.72(\mathrm{~d}, J=16.0 \mathrm{~Hz}, 1 \mathrm{H})$, $7.67(\mathrm{~d}, J=7.0 \mathrm{~Hz}, 3 \mathrm{H}), 7.56-7.48(\mathrm{~m}, 2 \mathrm{H}), 7.45-7.34(\mathrm{~m}, 5 \mathrm{H}), 7.23(\mathrm{t}, J=8.0 \mathrm{~Hz}$, $1 \mathrm{H}), 6.87-6.76(\mathrm{~m}, 2 \mathrm{H}), 6.58(\mathrm{~s}, 1 \mathrm{H}), 6.28(\mathrm{~d}, J=9.0 \mathrm{~Hz}, 1 \mathrm{H}), 3.77(\mathrm{~s}, 3 \mathrm{H}), 1.44(\mathrm{~s}$, 9H). ${ }^{13} \mathrm{C}\{1 \mathrm{H}\}$ NMR $\left(150 \mathrm{MHz}, \mathrm{CDCl}_{3}\right) \delta 177.5,160.3,158.3,155.5,155.1,139.0$, 135.4, 134.2, 134.0, 130.1, 129.8, 129.1, 128.2, 125.9, 125.0, 123.7, 120.4, 119.0, $117.8,117.2,112.1,110.7,91.4,80.0,79.0,55.8,40.3,28.5$. HRMS (FTMS-ESI), $\mathrm{m} / \mathrm{z}$ calcd for $\mathrm{C}_{32} \mathrm{H}_{29} \mathrm{NNaO}_{5}^{+}\left([\mathrm{M}+\mathrm{Na}]^{+}\right) 530.1938$, found 530.1948 .

$\mathrm{mV}$

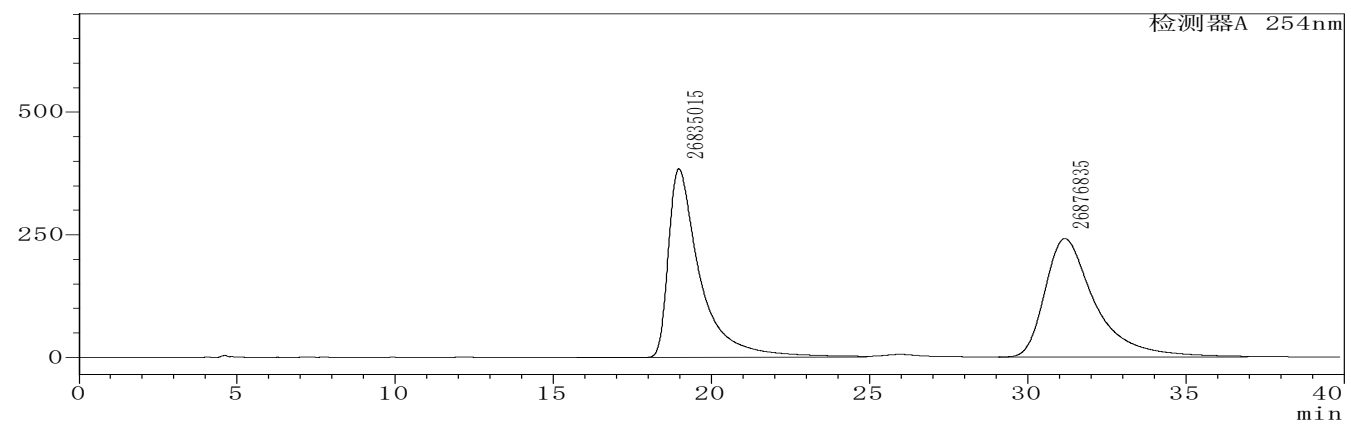

racemic

\begin{tabular}{|c|c|c|c|}
\hline & Retention Time & Area & \%Area \\
\hline 1 & 18.969 & 26835015 & 49.961 \\
\hline 2 & 31.176 & 26876835 & 50.039 \\
\hline
\end{tabular}

$\mathrm{mV}$

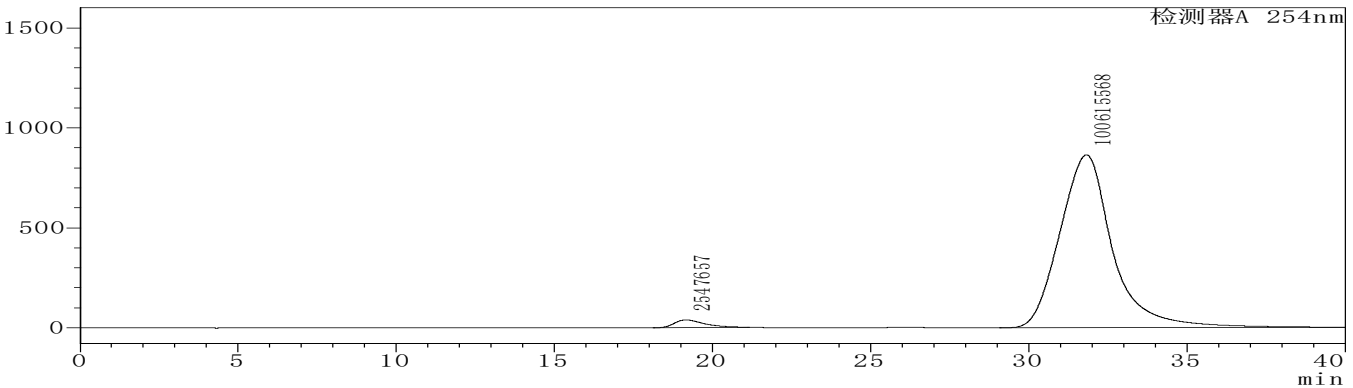

enantio-enriched

\begin{tabular}{|c|c|c|c|}
\hline & Retention Time & Area & \%Area \\
\hline 1 & 19.162 & 2547657 & 2.470 \\
\hline 2 & 31.825 & 100615568 & 97.530 \\
\hline
\end{tabular}


tert-Butyl $(S, E)$-(1-(2-(2-(naphthalen-2-yl)vinyl)-4-oxo-4H-chromen-3-yl)-3-phenylprop-2-yn-1-yl)carbamate (4o)<smiles>CC(C)(C)OC(=O)C[C@H](C#Cc1ccccc1)c1c(/C=C/c2ccc3ccccc3c2)oc2ccccc2c1=O</smiles>

Compound 4o: Prepared in $0.2 \mathrm{mmol}$ scale using 1.2 equiv of 1a at $70{ }^{\circ} \mathrm{C}$ for $72 \mathrm{~h} .82 \%$ yield $(86.7 \mathrm{mg})$, yellow solid; mp: $91.4-92.7{ }^{\circ} \mathrm{C}, 96 \%$ ee. HPLC (chiral ID column), hexane $/ i-\mathrm{PrOH}=70 / 30$, flow rate $0.8 \mathrm{ml} / \mathrm{min}, \lambda$ $=254 \mathrm{~nm}, t \mathrm{r}($ minor $)=14.42 \mathrm{~min}, t \mathrm{r}$ (major $)=21.07 \mathrm{~min}$. $[\alpha]^{25} \mathrm{D}=-227.6\left(\mathrm{c}=1.0\right.$ in $\left.\mathrm{CH}_{2} \mathrm{Cl}_{2}\right)$.

${ }^{1} \mathrm{H}$ NMR $\left(400 \mathrm{MHz}, \mathrm{CDCl}_{3}\right) \delta 8.20(\mathrm{~d}, J=7.8 \mathrm{~Hz}, 1 \mathrm{H})$, $8.03(\mathrm{~s}, 1 \mathrm{H}), 7.88(\mathrm{~d}, J=6.0 \mathrm{~Hz}, 2 \mathrm{H}), 7.86-7.80(\mathrm{~m}, 3 \mathrm{H}), 7.70(\mathrm{t}, J=7.8 \mathrm{~Hz}, 1 \mathrm{H})$, $7.60-7.48(\mathrm{~m}, 4 \mathrm{H}), 7.46-7.42(\mathrm{~m}, 2 \mathrm{H}), 7.39(\mathrm{t}, J=7.6 \mathrm{~Hz}, 1 \mathrm{H}), 7.26-7.22(\mathrm{~m}, 3 \mathrm{H})$, $6.74(\mathrm{~d}, J=7.8 \mathrm{~Hz}, 1 \mathrm{H}), 6.28(\mathrm{~d}, J=9.2 \mathrm{~Hz}, 1 \mathrm{H}), 1.48(\mathrm{~s}, 9 \mathrm{H}) .{ }^{13} \mathrm{C}\{1 \mathrm{H}\} \mathrm{NMR}(150$ $\left.\mathrm{MHz}, \mathrm{CDCl}_{3}\right) \delta 177.7,158.3,155.5,155.2,139.3,134.3,134.1,133.5,132.7,132.0$, $129.8,128.9,128.7,128.4,128.2,127.9,127.3,126.8,125.8,125.0,123.8,123.6$, 122.9, 118.6, 117.8, 116.8, 87.4, 82.5, 80.1, 40.3, 28.6. HRMS (FTMS-ESI), m/z calcd for $\mathrm{C}_{35} \mathrm{H}_{29} \mathrm{NNaO}_{4}{ }^{+}\left([\mathrm{M}+\mathrm{Na}]^{+}\right)$550.1989, found 550.1997.

$\mathrm{mV}$

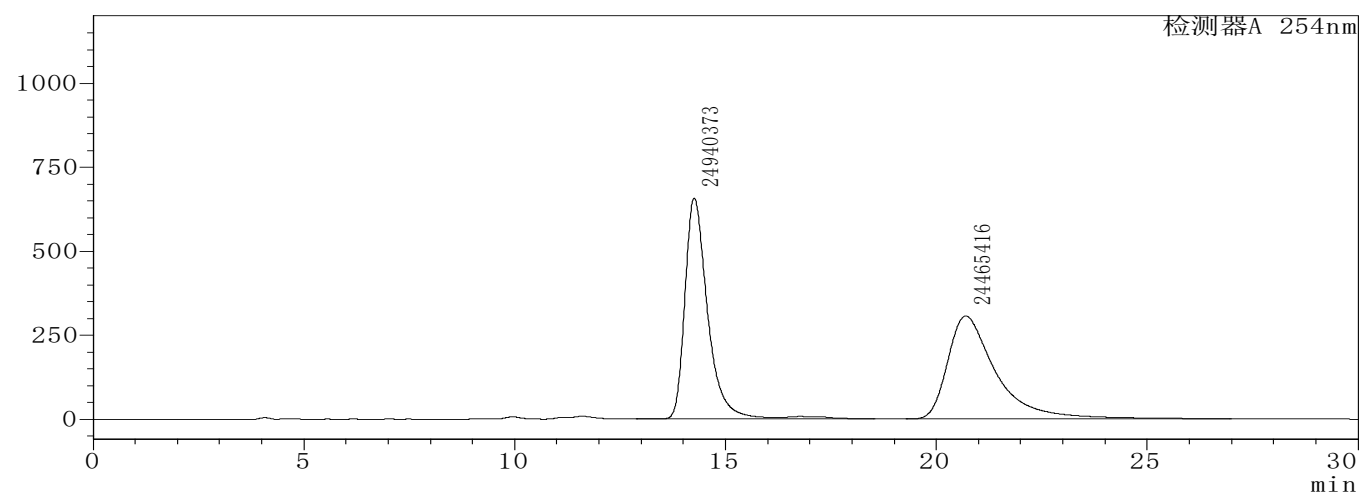

racemic

\begin{tabular}{|c|c|c|c|}
\hline & Retention Time & Area & \%Area \\
\hline 1 & 14.258 & 24940373 & 50.481 \\
\hline 2 & 20.698 & 24465416 & 49.519 \\
\hline
\end{tabular}

$\mathrm{mV}$

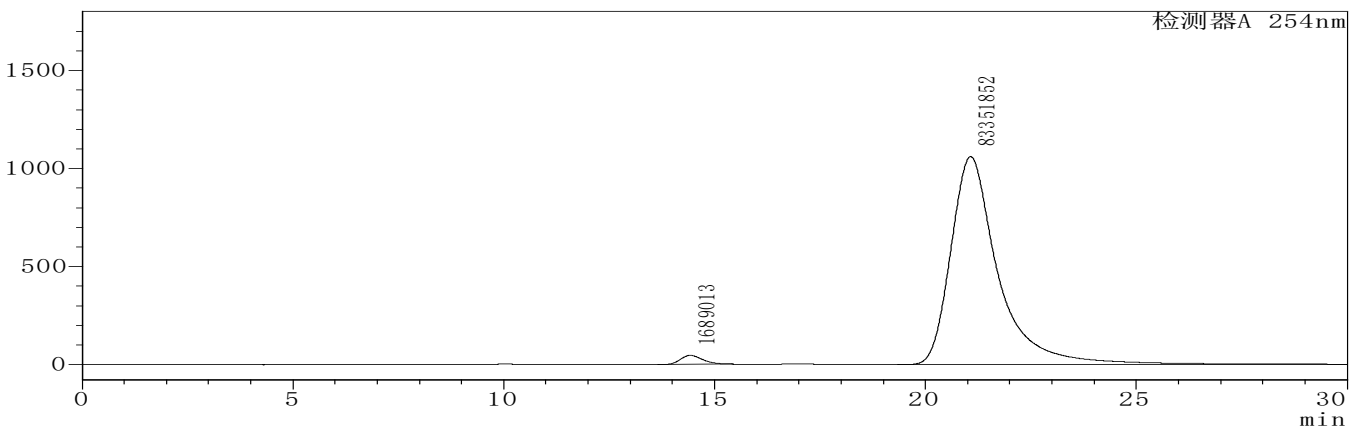

enantio-enriched

\begin{tabular}{|c|c|c|c|}
\hline & Retention Time & Area & \%Area \\
\hline 1 & 14.424 & 1689013 & 1.986 \\
\hline 2 & 21.072 & 83351852 & 98.014 \\
\hline
\end{tabular}


tert-Butyl (S,E)-(1-(2-(2-(furan-2-yl)vinyl)-4-oxo-4H-chromen-3-yl)-3-phenylprop-2-yn-1-yl)carbamate (4p)<smiles>CC(C)(C)OC(=O)C(C#Cc1ccco1)c1c(/C=C/c2ccccc2)oc2ccccc2c1=O</smiles>

Compound 4p: Prepared in $0.2 \mathrm{mmol}$ scale using 1.2 equiv of $1 \mathrm{a}$ at $70{ }^{\circ} \mathrm{C}$ for $72 \mathrm{~h} .75 \%$ yield $(70.0 \mathrm{mg}$ ), yellow solid; mp: 86.8-88.9 ${ }^{\circ} \mathrm{C}$, 95\% ee. HPLC (chiral ID column), hexane $/ i-\mathrm{PrOH}=70 / 30$, flow rate $0.8 \mathrm{ml} / \mathrm{min}, \lambda=254 \mathrm{~nm}, t \mathrm{r}$ $($ minor $)=13.38 \mathrm{~min}, t \mathrm{r}($ major $)=25.77 \mathrm{~min}$.

$[\alpha]^{25} \mathrm{D}=-181.7\left(\mathrm{c}=1.0\right.$ in $\left.\mathrm{CH}_{2} \mathrm{Cl}_{2}\right)$.

${ }^{1} \mathrm{H}$ NMR (400 MHz, $\left.\mathrm{cdcl}_{3}\right) \delta 8.18(\mathrm{~d}, J=7.8 \mathrm{~Hz}, 1 \mathrm{H}), 7.67(\mathrm{t}$, $J=7.8 \mathrm{~Hz}, 1 \mathrm{H}), 7.49(\mathrm{dd}, J=14.6,8.2 \mathrm{~Hz}, 3 \mathrm{H}), 7.44-7.40(\mathrm{~m}, 2 \mathrm{H}), 7.36(\mathrm{dd}, J=$ 15.0, 7.4 Hz, 2H), $7.30-7.22(\mathrm{~m}, 4 \mathrm{H}), 6.68(\mathrm{~d}, J=3.0 \mathrm{~Hz}, 1 \mathrm{H}), 6.50(\mathrm{~s}, 1 \mathrm{H}), 6.17(\mathrm{~d}$, $J=9.2 \mathrm{~Hz}, 1 \mathrm{H}), 1.45(\mathrm{~s}, 9 \mathrm{H}) .{ }^{13} \mathrm{C}\{1 \mathrm{H}\} \mathrm{NMR}\left(150 \mathrm{MHz}, \mathrm{CDCl}_{3}\right) \delta 177.6,158.2$, $155.4,155.2$, 151.8, 145.0, 134.0, 132.0, 128.3, 128.2, 125.9, 125.6, 125.0, 123.7, $122.9,118.5,117.7,114.7,114.6,112.7,87.4,82.5,80.0,40.2,28.5$. HRMS (FTMS-ESI), m/z calcd for $\mathrm{C}_{29} \mathrm{H}_{25} \mathrm{NNaO}_{5}{ }^{+}\left([\mathrm{M}+\mathrm{Na}]^{+}\right) 490.1625$, found 490.1633. $\mathrm{mV}$

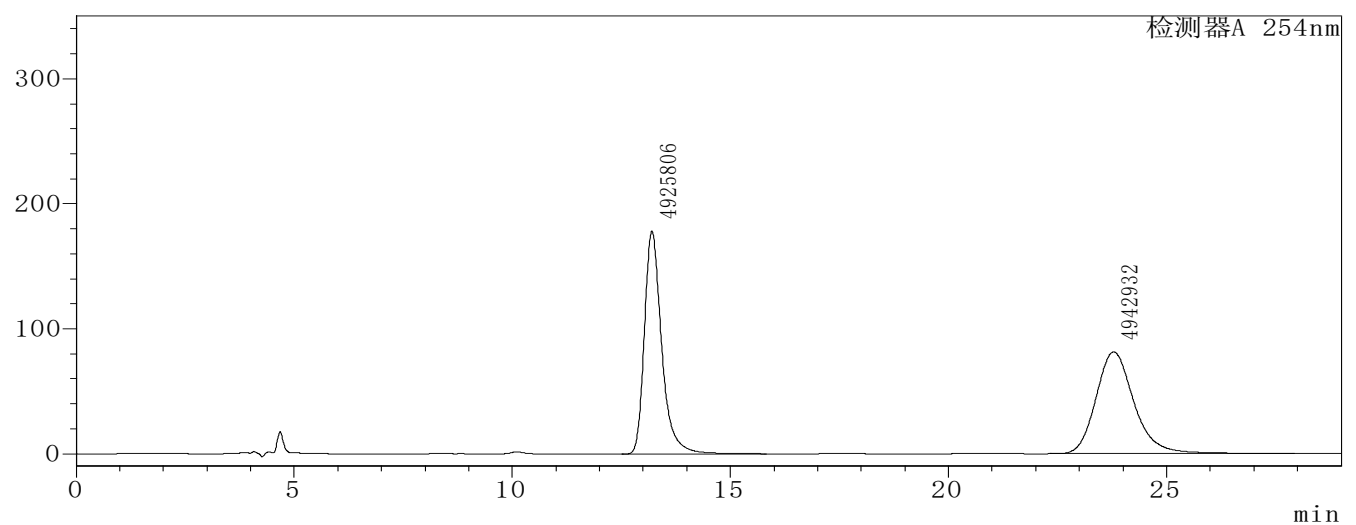

racemic

\begin{tabular}{|c|c|c|c|}
\hline & Retention Time & Area & \%Area \\
\hline 1 & 13.200 & 4925806 & 49.913 \\
\hline 2 & 23.786 & 4942932 & 50.087 \\
\hline
\end{tabular}

$\mathrm{mV}$

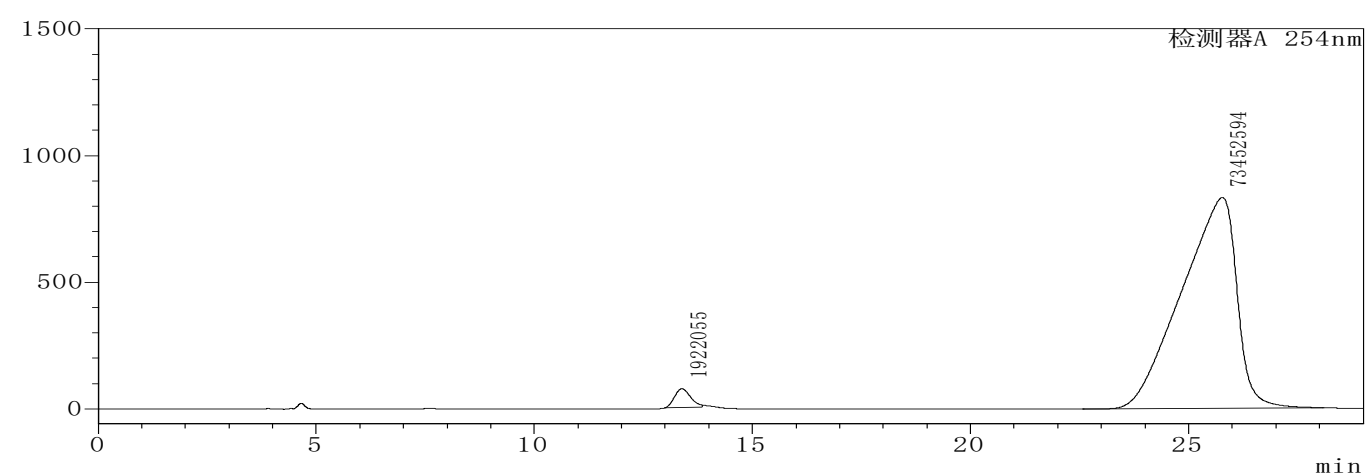

enantio-enriched

\begin{tabular}{|c|c|c|c|}
\hline & Retention Time & Area & \%Area \\
\hline 1 & 13.384 & 1922055 & 2.550 \\
\hline 2 & 25.772 & 73452594 & 97.450 \\
\hline
\end{tabular}


tert-Butyl $(S, E)$-(1-(4-oxo-2-(2-(thiophen-2-yl)vinyl)-4H-chromen-3-yl)-3-phenylprop-2-yn-1-yl)carbamate (4q)<smiles>CC(C)(C)OC(=O)C(C#Cc1cccs1)c1c(/C=C/c2ccccc2)oc2ccccc2c1=O</smiles>

Compound 4q: Prepared in $0.2 \mathrm{mmol}$ scale using 1.2 equiv of $1 \mathrm{a}$ at $70{ }^{\circ} \mathrm{C}$ for $72 \mathrm{~h} .73 \%$ yield $(70.3 \mathrm{mg})$, yellow solid; mp: 86.1-87.7 ${ }^{\circ} \mathrm{C}, 94 \%$ ee. HPLC (chiral IC column), hexane $/ i-\mathrm{PrOH}=90 / 10$, flow rate $0.8 \mathrm{ml} / \mathrm{min}, \lambda=254 \mathrm{~nm}, t \mathrm{r}$ $($ minor $)=21.46 \mathrm{~min}, \mathrm{tr}$ (major) $=31.79 \mathrm{~min}$.

$[\alpha]^{25} \mathrm{D}=-203.8\left(\mathrm{c}=1.0\right.$ in $\left.\mathrm{CH}_{2} \mathrm{Cl}_{2}\right)$.

${ }^{1} \mathrm{H}$ NMR $\left(400 \mathrm{MHz}, \mathrm{CDCl}_{3}\right) \delta 8.17(\mathrm{~d}, J=8.0 \mathrm{~Hz}, 1 \mathrm{H}), 7.82(\mathrm{~d}, J=15.4 \mathrm{~Hz}, 1 \mathrm{H})$, $7.66(\mathrm{t}, J=7.6 \mathrm{~Hz}, 1 \mathrm{H}), 7.49(\mathrm{~d}, J=8.4 \mathrm{~Hz}, 1 \mathrm{H}), 7.45-7.37(\mathrm{~m}, 3 \mathrm{H}), 7.37-7.31(\mathrm{~m}$, 2H), $7.28-7.22(\mathrm{~m}, 3 \mathrm{H}), 7.19(\mathrm{~d}, J=15.4 \mathrm{~Hz}, 1 \mathrm{H}), 7.09-7.05(\mathrm{~m}, 1 \mathrm{H}), 6.69(\mathrm{~d}, J=$ $8.0 \mathrm{~Hz}, 1 \mathrm{H}), 6.15(\mathrm{~d}, J=9.2 \mathrm{~Hz}, 1 \mathrm{H}), 1.46(\mathrm{~s}, 9 \mathrm{H}) .{ }^{13} \mathrm{C}\{1 \mathrm{H}\} \mathrm{NMR}\left(150 \mathrm{MHz}, \mathrm{CDCl}_{3}\right)$ $\delta 177.5,158.0,155.4,155.1,140.7,134.0,132.0,131.8,130.4,128.7,128.4,128.3$, 128.2, 125.8, 125.0, 123.6, 122.8, 118.2, 117.7, 115.5, 87.4, 82.5, 80.0, 40.1, 28.5. HRMS (FTMS-ESI), $\mathrm{m} / \mathrm{z}$ calcd for $\mathrm{C}_{29} \mathrm{H}_{25} \mathrm{NNaO}_{4} \mathrm{~S}^{+}\left([\mathrm{M}+\mathrm{Na}]^{+}\right) 506.1397$, found 506.1405 .

$\mathrm{mV}$

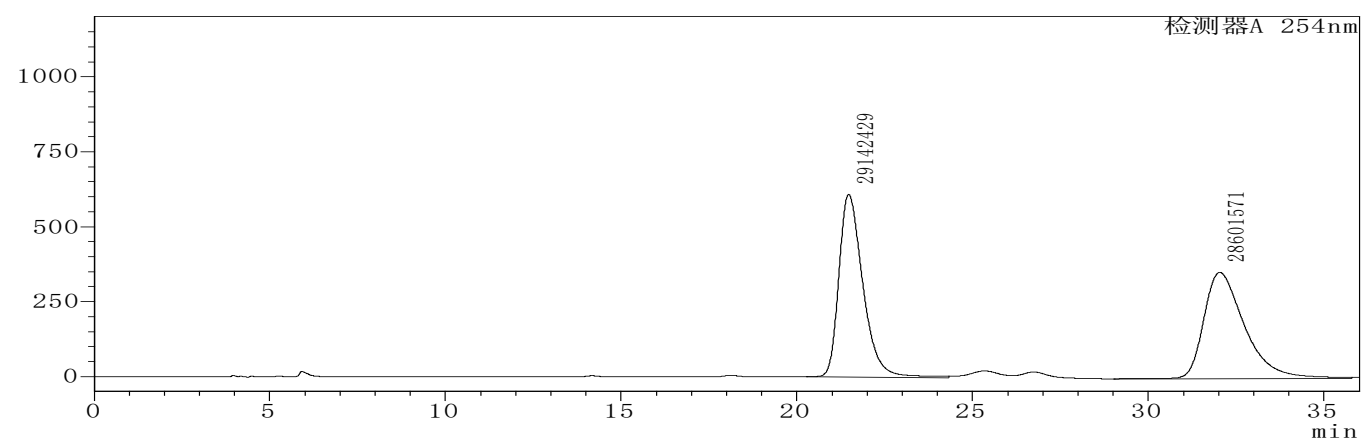

racemic

\begin{tabular}{|c|c|c|c|}
\hline & Retention Time & Area & \%Area \\
\hline 1 & 21.475 & 29142429 & 50.468 \\
\hline 2 & 32.034 & 28601571 & 49.532 \\
\hline
\end{tabular}

$\mathrm{mV}$

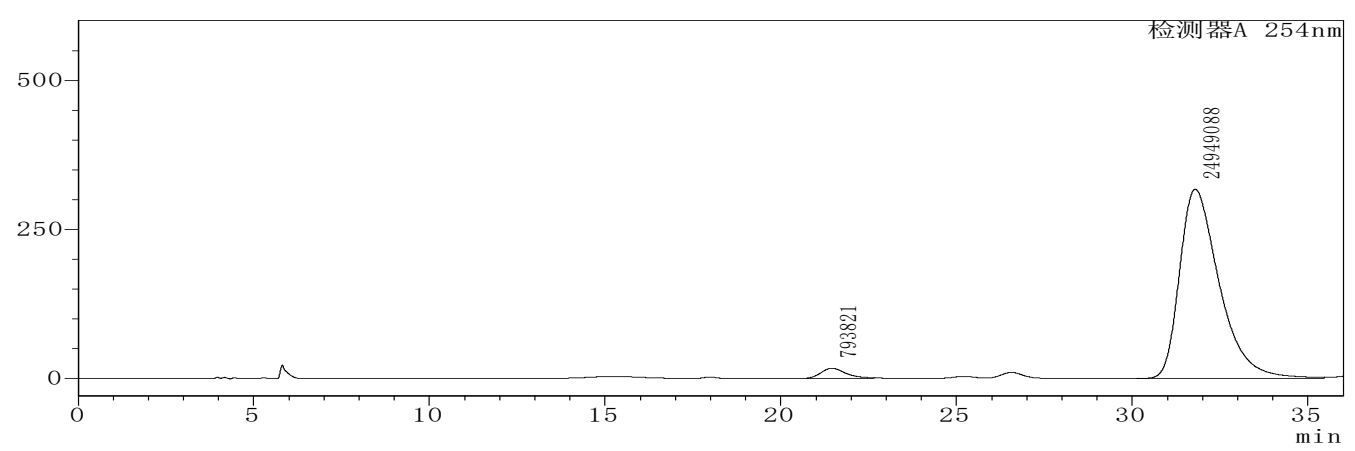

enantio-enriched

\begin{tabular}{|c|c|c|c|}
\hline & Retention Time & Area & \%Area \\
\hline 1 & 21.456 & 793821 & 3.084 \\
\hline 2 & 31.794 & 24949088 & 96.916 \\
\hline
\end{tabular}


tert-Butyl $(S, E)$-(3-cyclohexyl-1-(4-oxo-2-styryl-4H-chromen-3-yl)prop-2-yn-1yl)carbamate (4r)<smiles>CC(C)(C)OC(=O)[C@H](NC1CCCCC1)c1c(/C=C/c2ccccc2)oc2ccccc2c1=O</smiles>

Compound 4r: Prepared in $0.2 \mathrm{mmol}$ scale using 1.2 equiv of 1a at $70{ }^{\circ} \mathrm{C}$ for $48 \mathrm{~h} .73 \%$ yield $(70.6 \mathrm{mg})$, yellow solid; mp: $104.1-105.6{ }^{\circ} \mathrm{C}, 97 \%$ ee. HPLC (chiral ID column), hexane $/ i-\mathrm{PrOH}=70 / 30$, flow rate 0.8 $\mathrm{ml} / \mathrm{min}, \lambda=254 \mathrm{~nm}, \mathrm{tr}$ (minor) $=8.00 \mathrm{~min}, t \mathrm{r}$ (major) $=$ $9.88 \mathrm{~min}$.

$[\alpha]^{25}{ }_{\mathrm{D}}=-42.6\left(\mathrm{c}=1.0\right.$ in $\left.\mathrm{CH}_{2} \mathrm{Cl}_{2}\right)$.

${ }^{1} \mathrm{H}$ NMR $\left(400 \mathrm{MHz}, \mathrm{CDCl}_{3}\right) \delta 8.19(\mathrm{dd}, J=8.0,1.2 \mathrm{~Hz}, 1 \mathrm{H}), 7.74-7.63(\mathrm{~m}, 4 \mathrm{H})$, $7.51(\mathrm{~d}, J=8.4 \mathrm{~Hz}, 1 \mathrm{H}), 7.45-7.34(\mathrm{~m}, 5 \mathrm{H}), 6.49(\mathrm{~d}, J=6.0 \mathrm{~Hz}, 1 \mathrm{H}), 5.97(\mathrm{~d}, J=$ $8.8 \mathrm{~Hz}, 1 \mathrm{H}), 2.43-2.26(\mathrm{~m}, 1 \mathrm{H}), 1.83-1.69(\mathrm{~m}, 2 \mathrm{H}), 1.70-1.57(\mathrm{~m}, 2 \mathrm{H}), 1.55-$ $1.31(\mathrm{~m}, 12 \mathrm{H}), 1.31-1.14(\mathrm{~m}, 3 \mathrm{H}) .{ }^{13} \mathrm{C}\{1 \mathrm{H}\} \mathrm{NMR}\left(150 \mathrm{MHz}, \mathrm{CDCl}_{3}\right) \delta 177.6,158.0$, $155.5,155.2$, 138.8, 135.4, 133.9, 130.1, 129.1, 128.1, 125.9, 124.9, 123.7, 119.4, 117.8, 117.1, 87.3, 79.8, 78.0, 39.8, 32.6, 29.2, 28.5, 26.0, 24.9. HRMS (FTMS-ESI), $\mathrm{m} / \mathrm{z}$ calcd for $\mathrm{C}_{31} \mathrm{H}_{33} \mathrm{NNaO}_{4}{ }^{+}\left([\mathrm{M}+\mathrm{Na}]^{+}\right)$506.2302, found 506.2310.

$\mathrm{mV}$

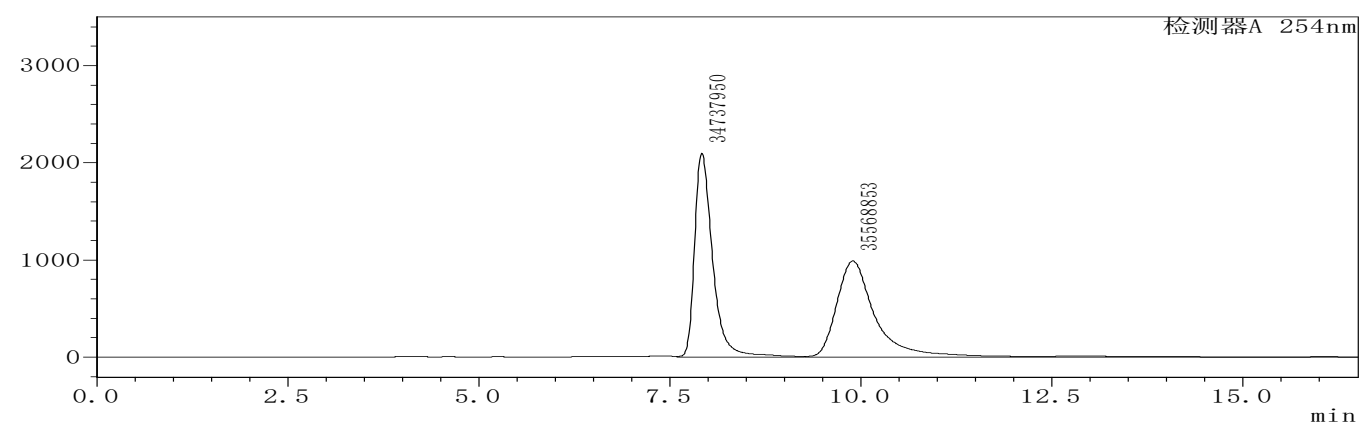

racemic

\begin{tabular}{|c|c|c|c|}
\hline & Retention Time & Area & \%Area \\
\hline 1 & 7.917 & 34737950 & 49.409 \\
\hline 2 & 9.893 & 35568853 & 50.591 \\
\hline
\end{tabular}

$\mathrm{mV}$

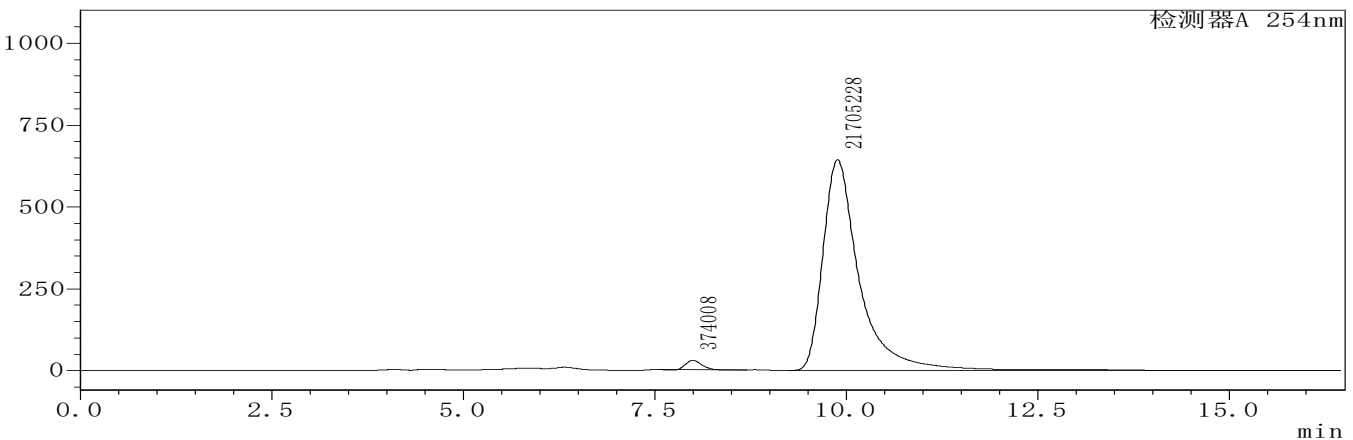

enantio-enriched

\begin{tabular}{|c|c|c|c|}
\hline & Retention Time & Area & \%Area \\
\hline 1 & 7.995 & 374008 & 1.694 \\
\hline 2 & 9.883 & 21705228 & 98.306 \\
\hline
\end{tabular}


Benzyl (R)-2-(((benzyloxy)carbonyl)amino)-2-(2-methyl-4-oxo-4H-chromen-3-yl) acetate $(6 a)$<smiles>Cc1oc2ccccc2c(=O)c1[C@H](NC(=O)OCc1ccccc1)C(=O)OCc1ccccc1</smiles>

Compound 6a: Prepared in $0.2 \mathrm{mmol}$ scale using 1.2 equiv of 5a at $26^{\circ} \mathrm{C}$ for 27 h. $59 \%$ yield $(54.0 \mathrm{mg})$, colorless oil, $86 \%$ $e e$. HPLC (chiral IB column), hexane $/ i-\mathrm{PrOH}=80 / 20$, flow rate $0.8 \mathrm{ml} / \mathrm{min}, \lambda=254 \mathrm{~nm}, t \mathrm{r}$ (minor) $=15.11 \mathrm{~min}, t \mathrm{r}$ (major)

$=16.54 \mathrm{~min}$.

$[\alpha]^{25} \mathrm{D}=-65.1\left(\mathrm{c}=1.0\right.$ in $\left.\mathrm{CH}_{2} \mathrm{Cl}_{2}\right)$.

${ }^{1} \mathrm{H}$ NMR $\left(400 \mathrm{MHz}, \mathrm{CDCl}_{3}\right) \delta 8.13(\mathrm{~d}, J=8.0 \mathrm{~Hz}, 1 \mathrm{H}), 7.69-7.62(\mathrm{~m}, 1 \mathrm{H}), 7.44-$ $7.35(\mathrm{~m}, 2 \mathrm{H}), 7.35-7.26(\mathrm{~m}, 10 \mathrm{H}), 6.50(\mathrm{~d}, J=9.0 \mathrm{~Hz}, 1 \mathrm{H}), 5.48(\mathrm{~d}, J=9.0 \mathrm{~Hz}, 1 \mathrm{H})$, $5.24(\mathrm{~d}, J=12.4 \mathrm{~Hz}, 1 \mathrm{H}), 5.17(\mathrm{~d}, J=7.0 \mathrm{~Hz}, 1 \mathrm{H}), 5.13(\mathrm{~d}, J=7.0 \mathrm{~Hz}, 1 \mathrm{H}), 5.07(\mathrm{~d}, J$ $=12.4 \mathrm{~Hz}, 1 \mathrm{H}), 2.67(\mathrm{~s}, 3 \mathrm{H}) .{ }^{13} \mathrm{C}\{1 \mathrm{H}\} \mathrm{NMR}\left(100 \mathrm{MHz}, \mathrm{CDCl}_{3}\right) \delta 177.1,169.8,165.3$, $156.3,156.1,136.2,135.4,133.9,128.5,128.2$, 128.1, 128.1, 127.9, 125.6, 125.2, 123.0, 119.2, 117.9, 67.6, 67.0, 52.0, 18.6. HRMS (FTMS-ESI), m/z calcd for $\mathrm{C}_{27} \mathrm{H}_{24} \mathrm{NO}_{6}{ }^{+}\left([\mathrm{M}+\mathrm{H}]^{+}\right) 458.1598$, found 458.1596.

$\mathrm{mV}$

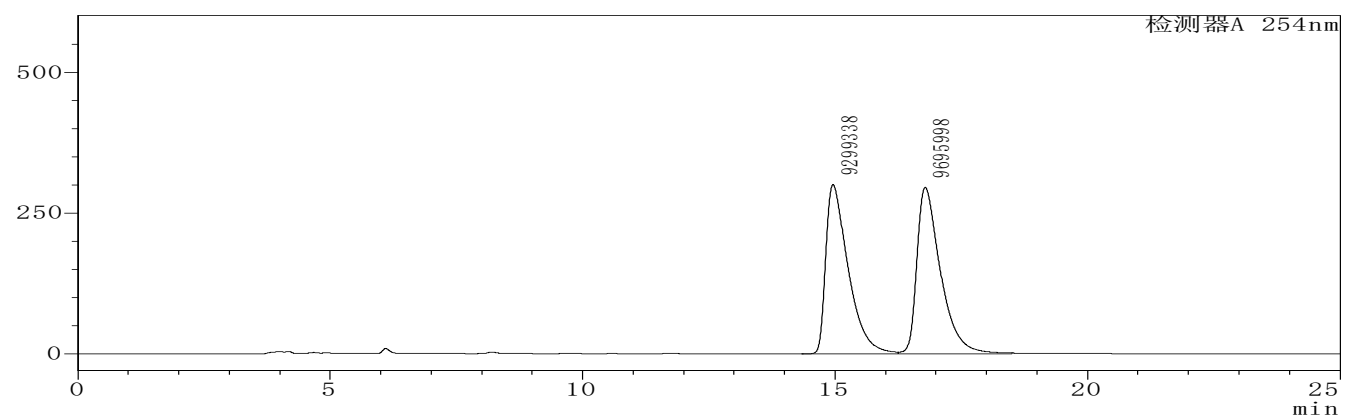

racemic

\begin{tabular}{|c|c|c|c|}
\hline & Retention Time & Area & \%Area \\
\hline 1 & 14.961 & 9299338 & 48.956 \\
\hline 2 & 16.786 & 9695998 & 51.044 \\
\hline
\end{tabular}

$\mathrm{mV}$

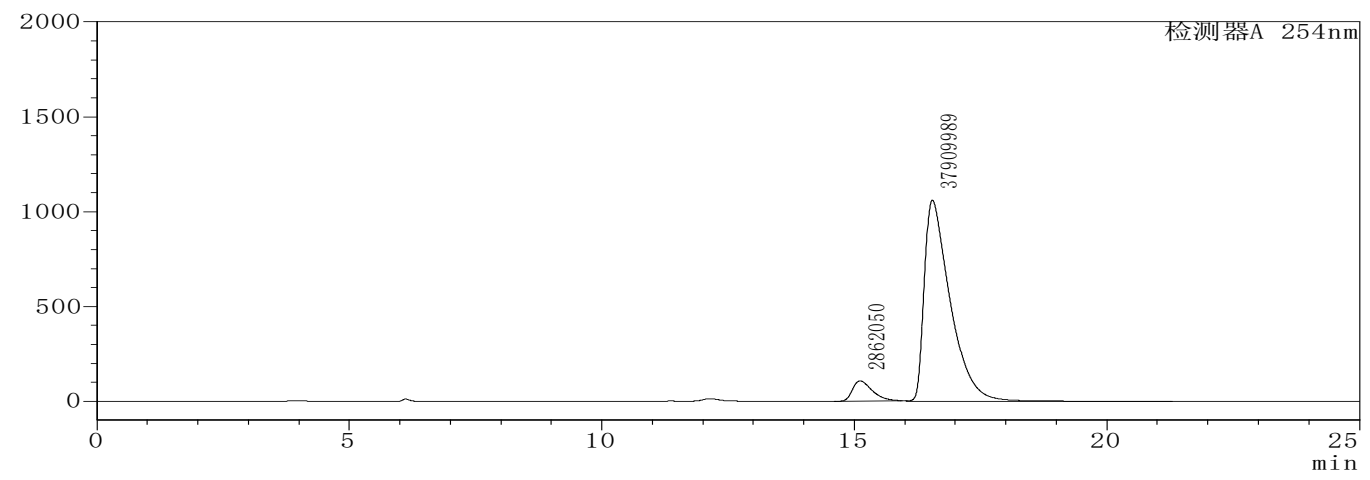

enantio-enriched

\begin{tabular}{|c|c|c|c|}
\hline & Retention Time & Area & \%Area \\
\hline 1 & 15.114 & 2862050 & 7.020 \\
\hline 2 & 16.542 & 37909989 & 92.980 \\
\hline
\end{tabular}


Benzyl $(R)-2-(((b e n z y l o x y)$ carbonyl)amino)-2-(4-oxo-2-phenethyl-4H-chromen-3yl)acetate $(6 b)$

Compound 6b: Prepared in $0.2 \mathrm{mmol}$ scale using 1.2 equiv of
$\begin{aligned} & \text { 5a at } 26{ }^{\circ} \mathrm{C} \text { for } 27 \mathrm{~h} .61 \% \text { yield }(67.0 \mathrm{mg}), \text { colorless oil, } 78 \% \\ & \text { ee. } \mathrm{HPLC} \text { (chiral } \mathrm{IB} \text { column), hexane } / i-\mathrm{PrOH}=70 / 30, \text { flow } \\ & \text { rate } 0.8 \mathrm{ml} / \mathrm{min}, \lambda=254 \mathrm{~nm}, t \mathrm{r} \text { (minor) }=10.59 \mathrm{~min}, \mathrm{tr} \text { (major) }\end{aligned}$ $=12.18 \mathrm{~min}$.

$[\alpha]^{25}=-40.7\left(\mathrm{c}=1.0\right.$ in $\left.\mathrm{CH}_{2} \mathrm{Cl}_{2}\right)$.

${ }^{1} \mathrm{H}$ NMR $\left(400 \mathrm{MHz}, \mathrm{CDCl}_{3}\right) \delta 8.13(\mathrm{~d}, J=7.8 \mathrm{~Hz}, 1 \mathrm{H}), 7.69-7.61(\mathrm{~m}, 1 \mathrm{H}), 7.41-$ $7.16(\mathrm{~m}, 17 \mathrm{H}), 6.43(\mathrm{~d}, J=9.0 \mathrm{~Hz}, 1 \mathrm{H}), 5.53(\mathrm{~d}, J=9.0 \mathrm{~Hz}, 1 \mathrm{H}), 5.22(\mathrm{~d}, J=12.4 \mathrm{~Hz}$, $1 \mathrm{H}), 5.16(\mathrm{~d}, J=4.0 \mathrm{~Hz}, 1 \mathrm{H}), 5.13(\mathrm{~d}, J=4.0 \mathrm{~Hz}, 1 \mathrm{H}), 5.08(\mathrm{~d}, J=12.4 \mathrm{~Hz}, 1 \mathrm{H}), 3.37$ $(\mathrm{dt}, J=19.6,8.0 \mathrm{~Hz}, 1 \mathrm{H}), 3.21-3.05(\mathrm{~m}, 3 \mathrm{H}) .{ }^{13} \mathrm{C}\{1 \mathrm{H}\} \mathrm{NMR}\left(100 \mathrm{MHz}, \mathrm{CDCl}_{3}\right) \delta$ $177.6,169.9,167.5,156.3,156.2,139.9,136.3,135.5,134.0,128.7,128.6,128.5$, 128.4, 128.2, 128.2, 128.0, 126.6, 125.7, 125.3, 123.0, 119.2, 117.9, 67.8, 67.2, 51.7, 34.2, 33.9. HRMS (FTMS-ESI), $\mathrm{m} / \mathrm{z}$ calcd for $\mathrm{C}_{34} \mathrm{H}_{30} \mathrm{NO}_{6}{ }^{+}\left([\mathrm{M}+\mathrm{H}]^{+}\right) 548.2068$, found 548.2067.

$\mathrm{mV}$

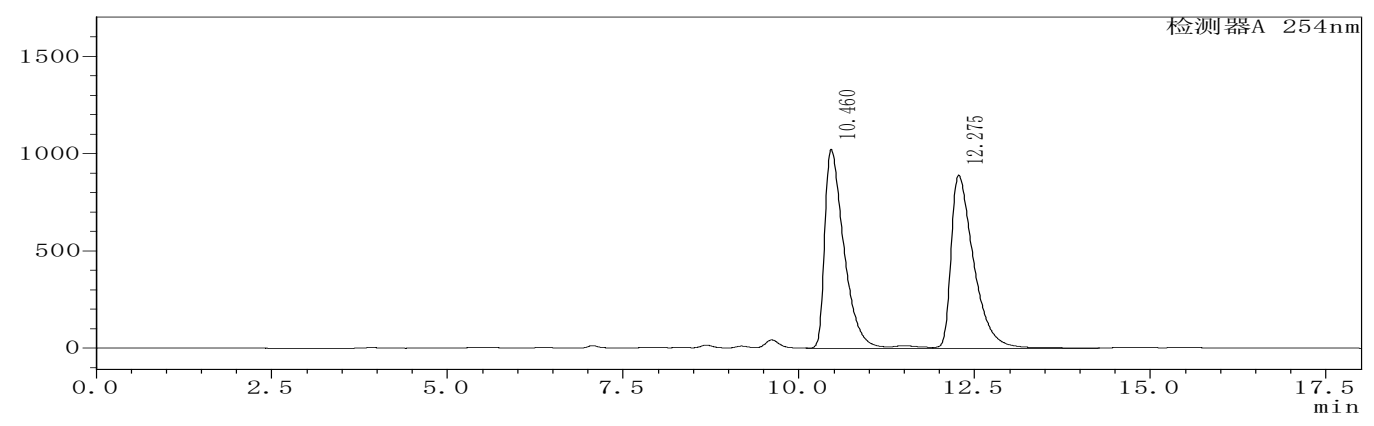

racemic

\begin{tabular}{|c|c|c|c|}
\hline & Retention Time & Area & \%Area \\
\hline 1 & 10.460 & 19726164 & 49.642 \\
\hline 2 & 12.275 & 20010537 & 50.358 \\
\hline
\end{tabular}

$\mathrm{mV}$

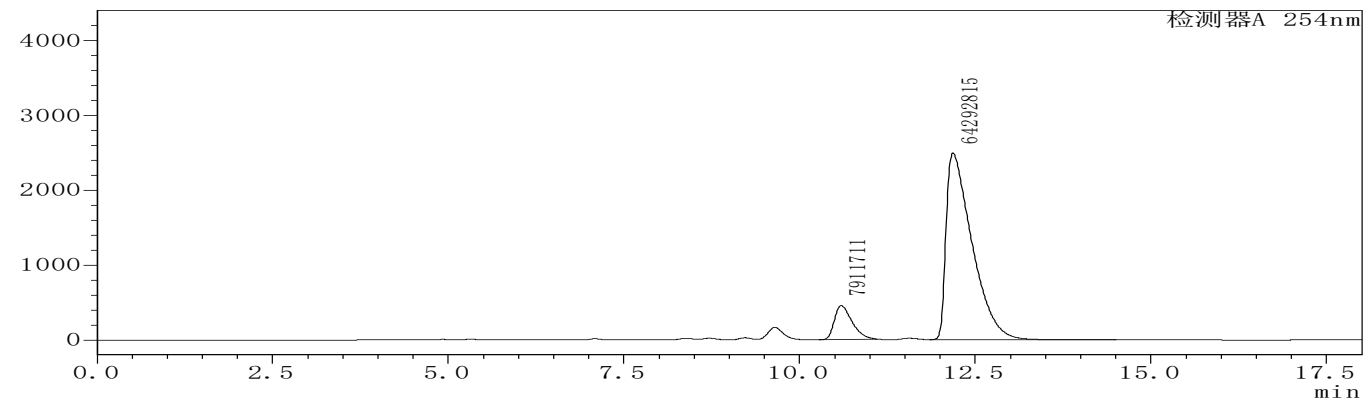

enantio-enriched

\begin{tabular}{|c|c|c|c|}
\hline & Retention Time & Area & \%Area \\
\hline 1 & 10.592 & 7911711 & 10.957 \\
\hline 2 & 12.183 & 64292815 & 89.043 \\
\hline
\end{tabular}


Benzyl (R)-2-(((benzyloxy)carbonyl)amino)-2-(2-(methoxymethyl)-4-oxo-4H-chromen-3-yl)acetate $(6 c)$<smiles>COCc1oc2ccccc2c(=O)c1[C@H](NC(=O)OCc1ccccc1)C(=O)O</smiles>

Compound 6c: Prepared in $0.2 \mathrm{mmol}$ scale using 1.2 equiv of 5a at $26^{\circ} \mathrm{C}$ for $27 \mathrm{~h} .47 \%$ yield $(45.5 \mathrm{mg})$, colorless oil, $89 \%$ $e e$. HPLC (chiral IB column), hexane $/ i-\mathrm{PrOH}=80 / 20$, flow rate $0.8 \mathrm{ml} / \mathrm{min}, \lambda=254 \mathrm{~nm}, \operatorname{tr}($ minor $)=13.50 \mathrm{~min}, \operatorname{tr}$ (major)

$=14.05 \mathrm{~min}$.

$[\alpha]^{25}=-52.7\left(\mathrm{c}=1.0\right.$ in $\left.\mathrm{CH}_{2} \mathrm{Cl}_{2}\right)$.

${ }^{1} \mathrm{H}$ NMR $\left(400 \mathrm{MHz}, \mathrm{CDCl}_{3}\right) \delta 8.14(\mathrm{~d}, J=8.0 \mathrm{~Hz}, 1 \mathrm{H}), 7.69(\mathrm{t}, J=7.8 \mathrm{~Hz}, 1 \mathrm{H}), 7.48$ $(\mathrm{d}, J=8.4 \mathrm{~Hz}, 1 \mathrm{H}), 7.40(\mathrm{t}, J=7.6 \mathrm{~Hz}, 1 \mathrm{H}), 7.33-7.21(\mathrm{~m}, 10 \mathrm{H}), 6.37(\mathrm{~d}, J=8.4 \mathrm{~Hz}$, $1 \mathrm{H}), 5.74(\mathrm{~d}, J=8.6 \mathrm{~Hz}, 1 \mathrm{H}), 5.26(\mathrm{~d}, J=12.4 \mathrm{~Hz}, 1 \mathrm{H}), 5.12(\mathrm{~d}, J=12.4 \mathrm{~Hz}, 2 \mathrm{H})$, $5.04(\mathrm{~d}, J=12.4 \mathrm{~Hz}, 1 \mathrm{H}), 4.97(\mathrm{~d}, J=13.2 \mathrm{~Hz}, 1 \mathrm{H}), 4.49$ (d, $J=13.2 \mathrm{~Hz}, 1 \mathrm{H}), 3.41$ (s, $3 \mathrm{H}) .{ }^{13} \mathrm{C}\{1 \mathrm{H}\} \mathrm{NMR}\left(100 \mathrm{MHz}, \mathrm{CDCl}_{3}\right) \delta 177.5,169.8,163.1,156.2,156.1,136.3$, 135.5, 134.3, 128.5, 128.3, 128.1, 128.0, 125.8, 125.5, 123.2, 120.9, 118.3, 70.0, 67.7, 67.1, 59.0, 50.7. HRMS (FTMS-ESI), m/z calcd for $\mathrm{C}_{28} \mathrm{H}_{26} \mathrm{NO}_{7}^{+}\left([\mathrm{M}+\mathrm{H}]^{+}\right) 488.1704$, found 488.1707 .

$\mathrm{mV}$

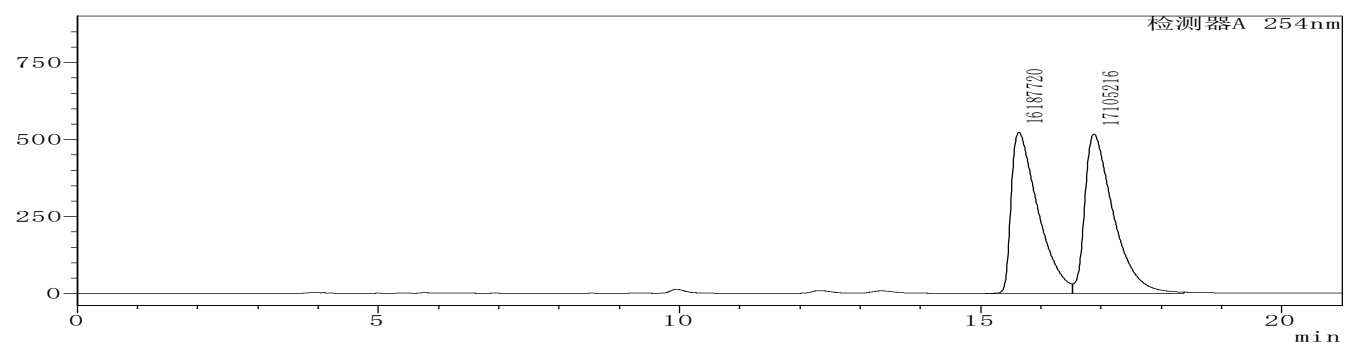

racemic

\begin{tabular}{|c|c|c|c|}
\hline & Retention Time & Area & \%Area \\
\hline 1 & 15.635 & 16187720 & 48.622 \\
\hline 2 & 16.882 & 17105216 & 51.378 \\
\hline
\end{tabular}

$\mathrm{mV}$

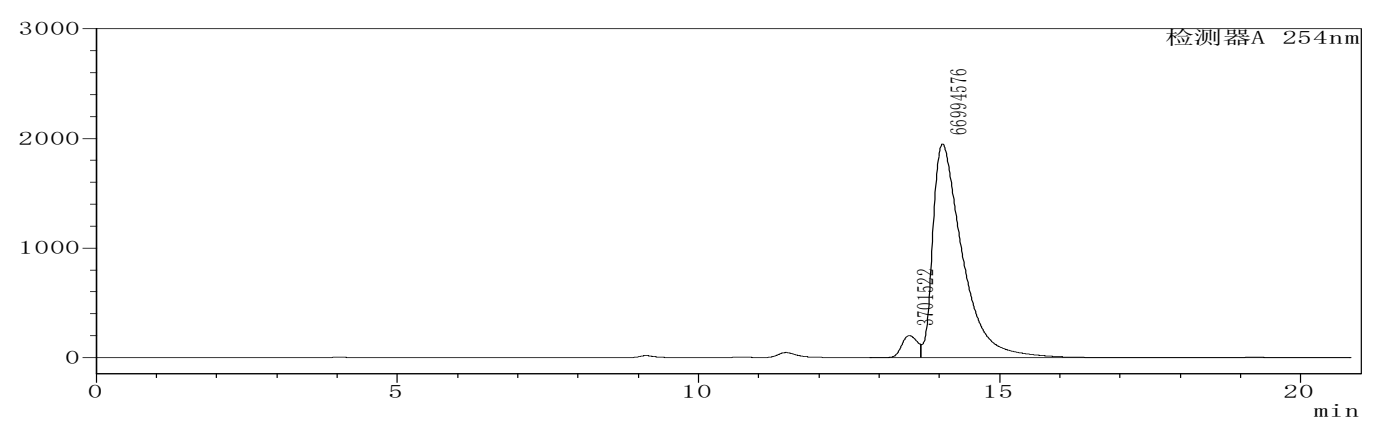

enantio-enriched

\begin{tabular}{|c|c|c|c|}
\hline & Retention Time & Area & \%Area \\
\hline 1 & 13.502 & 3701522 & 5.236 \\
\hline 2 & 14.054 & 66994576 & 94.764 \\
\hline
\end{tabular}


Benzyl (R)-2-(((benzyloxy)carbonyl)amino)-2-(4-0xo-2-propyl-4H-chromen-3-yl) Acetate (6d)<smiles>CCCc1oc2ccccc2c(=O)c1[C@H](NC(=O)OCc1ccccc1)C(=O)O</smiles>

Compound 6d: Prepared in $0.2 \mathrm{mmol}$ scale using 1.2 equiv of 5a at $26^{\circ} \mathrm{C}$ for 27 h. $44 \%$ yield (43.0 mg), colorless oil, $73 \%$ $e e$. HPLC (chiral IA column), hexane $i-\mathrm{PrOH}=70 / 30$, flow rate $0.8 \mathrm{ml} / \mathrm{min}, \lambda=254 \mathrm{~nm}, \operatorname{tr}$ (minor) $=21.16 \mathrm{~min}, \operatorname{tr}$ (major)

$=23.57 \mathrm{~min}$.

$[\alpha]^{25}=-51.2\left(\mathrm{c}=1.0\right.$ in $\left.\mathrm{CH}_{2} \mathrm{Cl}_{2}\right)$.

${ }^{1} \mathrm{H}$ NMR $\left(400 \mathrm{MHz}, \mathrm{CDCl}_{3}\right) \delta 8.12(\mathrm{~d}, J=8.0 \mathrm{~Hz}, 1 \mathrm{H}), 7.66(\mathrm{t}, J=7.8 \mathrm{~Hz}, 1 \mathrm{H}), 7.43$ $(\mathrm{d}, J=8.4 \mathrm{~Hz}, 1 \mathrm{H}), 7.38(\mathrm{t}, J=7.6 \mathrm{~Hz}, 1 \mathrm{H}), 7.34-7.22(\mathrm{~m}, 10 \mathrm{H}), 6.45(\mathrm{~d}, J=9.2 \mathrm{~Hz}$, $1 \mathrm{H}), 5.49(\mathrm{~d}, J=9.2 \mathrm{~Hz}, 1 \mathrm{H}), 5.24(\mathrm{~d}, J=12.4 \mathrm{~Hz}, 1 \mathrm{H}), 5.14(\mathrm{~d}, J=6.2 \mathrm{~Hz}, 1 \mathrm{H}), 5.10$ $(\mathrm{d}, J=6.2 \mathrm{~Hz}, 1 \mathrm{H}), 5.07(\mathrm{~d}, J=12.4 \mathrm{~Hz}, 1 \mathrm{H}), 3.11-2.98(\mathrm{~m}, 1 \mathrm{H}), 2.86-2.74(\mathrm{~m}$, 1H), $1.82(\mathrm{dd}, J=14.6,7.2 \mathrm{~Hz}, 2 \mathrm{H}), 1.01(\mathrm{t}, J=7.4 \mathrm{~Hz}, 3 \mathrm{H}) .{ }^{13} \mathrm{C}\{1 \mathrm{H}\} \mathrm{NMR}(100$ $\left.\mathrm{MHz}, \mathrm{CDCl}_{3}\right) \delta 177.6,170.0,168.6,156.4,156.3,136.4,135.5,134.0,128.6,128.6$, 128.3, 128.2, 128.0, 125.7, 125.2, 123.0, 119.1, 118.0, 67.7, 67.1, 51.81, 34.0, 21.4, 13.9. HRMS (FTMS-ESI), m/z calcd for $\mathrm{C}_{29} \mathrm{H}_{27} \mathrm{NO}_{6}{ }^{+}\left([\mathrm{M}+\mathrm{Na}]^{+}\right) 508.1731$, found 508.1739 .

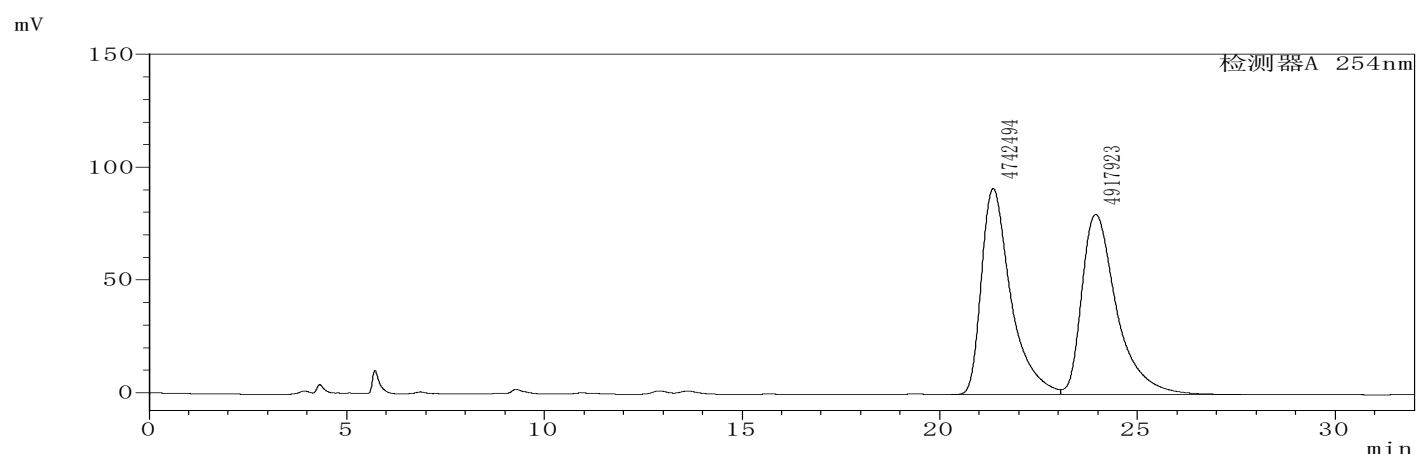

racemic

\begin{tabular}{|c|c|c|c|}
\hline & Retention Time & Area & \%Area \\
\hline 1 & 21.352 & 4742494 & 49.092 \\
\hline 2 & 23.948 & 4917923 & 50.908 \\
\hline
\end{tabular}

$\mathrm{mV}$

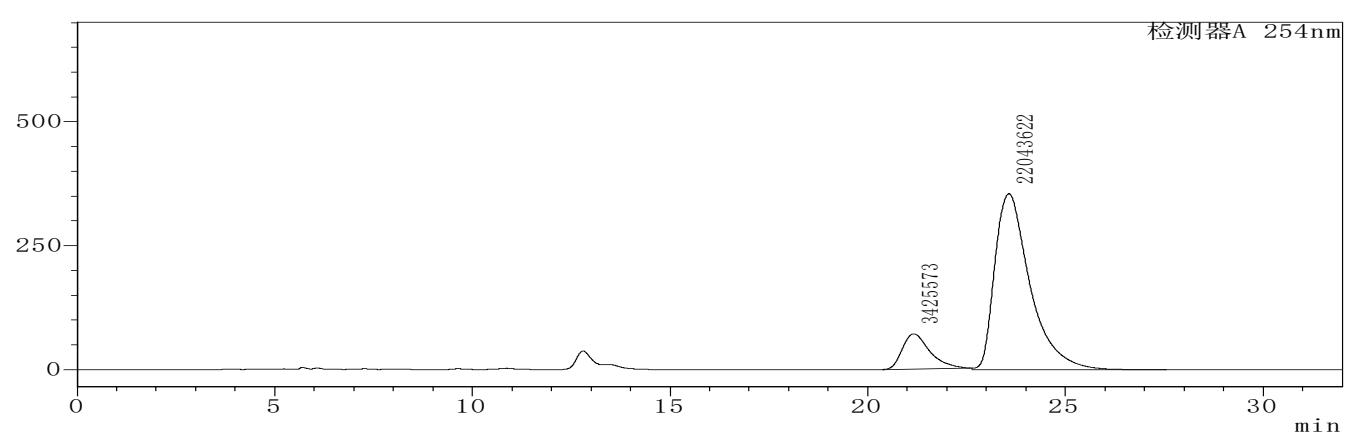

enantio-enriched

\begin{tabular}{|c|c|c|c|}
\hline & Retention Time & Area & \%Area \\
\hline 1 & 21.163 & 3425573 & 13.450 \\
\hline 2 & 23.570 & 22043622 & 86.550 \\
\hline
\end{tabular}


Benzyl $(R, E)-2-(((b e n z y l o x y)$ carbonyl)amino)-2-(4-oxo-2-styryl-4H-chromen-3yl)acetate $(6 \mathrm{e})$

$\mathrm{O} \quad \mathrm{NHCO}_{2} \mathrm{Bn}$ Compound 6e: Prepared in $0.2 \mathrm{mmol}$ scale using 1.2 equiv of $\mathrm{CO}_{2} \mathrm{Bn} \quad \mathbf{5 a}$ at $26^{\circ} \mathrm{C}$ for $27 \mathrm{~h} .37 \%$ yield $(40.6 \mathrm{mg})$, white solid; mp: 123.6-125.3 ${ }^{\circ} \mathrm{C}, 78 \%$ ee. HPLC (chiral IA column), hexane $/ i-\mathrm{PrOH}=70 / 30$, flow rate $0.8 \mathrm{ml} / \mathrm{min}, \lambda=254 \mathrm{~nm}, t \mathrm{r}$ $($ major $)=35.51 \mathrm{~min}, t \mathrm{r}($ minor $)=44.57 \mathrm{~min}$.

$[\alpha]^{25} \mathrm{D}=-4.9\left(\mathrm{c}=1.0\right.$ in $\left.\mathrm{CH}_{2} \mathrm{Cl}_{2}\right)$.

${ }^{1} \mathrm{H}$ NMR $\left(400 \mathrm{MHz}, \mathrm{CDCl}_{3}\right) \delta 8.13(\mathrm{~d}, J=7.8 \mathrm{~Hz}, 1 \mathrm{H}), 7.73(\mathrm{~d}, J=15.8 \mathrm{~Hz}, 1 \mathrm{H})$, $7.67(\mathrm{dd}, J=12.8,7.8 \mathrm{~Hz}, 3 \mathrm{H}), 7.57-7.49(\mathrm{~m}, 2 \mathrm{H}), 7.45-7.36(\mathrm{~m}, 4 \mathrm{H}), 7.34-7.21$ $(\mathrm{m}, 10 \mathrm{H}), 6.47(\mathrm{~d}, J=9.2 \mathrm{~Hz}, 1 \mathrm{H}), 5.77(\mathrm{~d}, J=9.2 \mathrm{~Hz}, 1 \mathrm{H}), 5.22(\mathrm{~d}, J=12.4 \mathrm{~Hz}, 1 \mathrm{H})$, $5.17(\mathrm{~d}, J=8.0 \mathrm{~Hz}, 1 \mathrm{H}), 5.14(\mathrm{~d}, J=8.0 \mathrm{~Hz}, 1 \mathrm{H}), 5.09(\mathrm{~d}, J=12.2 \mathrm{~Hz}, 1 \mathrm{H}) .{ }^{13} \mathrm{C}\{1 \mathrm{H}\}$ NMR $\left(100 \mathrm{MHz}, \mathrm{CDCl}_{3}\right) \delta 177.8,170.0,160.0,156.4,155.7,139.5,136.3,135.5$, $135.3,134.3,130.2,129.1,128.6,128.5,128.3$, 128.2, 128.0, 125.8, 125.2, 123.1, 118.6, 117.9, 117.1, 67.7, 67.2, 51.1. HRMS (FTMS-ESI), $\mathrm{m} / \mathrm{z}$ calcd for $\mathrm{C}_{34} \mathrm{H}_{27} \mathrm{NNaO}_{6}{ }^{+}\left([\mathrm{M}+\mathrm{Na}]^{+}\right)$568.1731, found 568.1733.

$\mathrm{mV}$

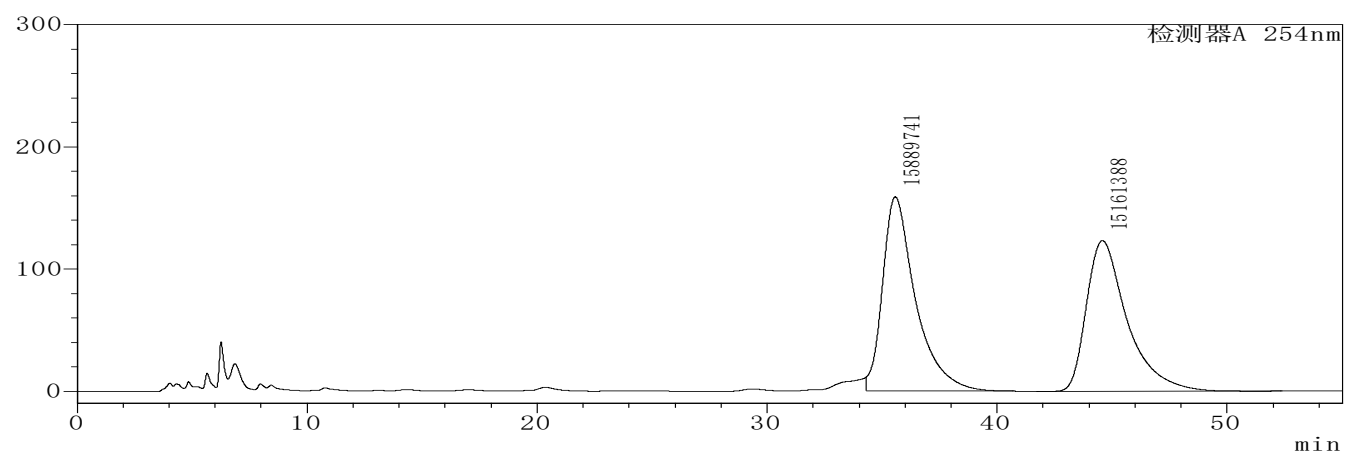

racemic

\begin{tabular}{|c|c|c|c|}
\hline & Retention Time & Area & \%Area \\
\hline 1 & 35.574 & 15889741 & 51.173 \\
\hline 2 & 44.580 & 15161388 & 48.827 \\
\hline
\end{tabular}

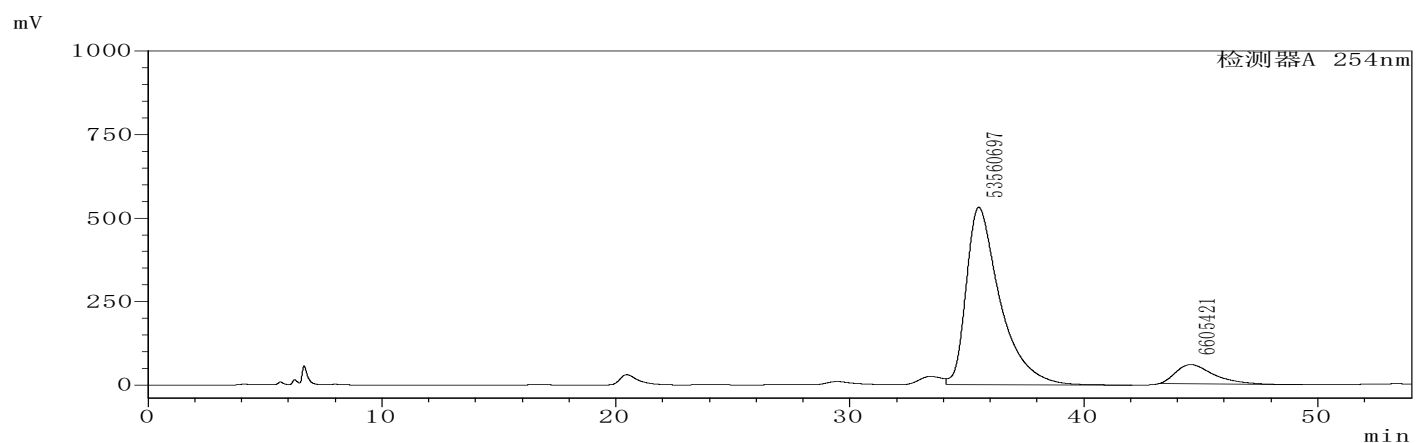

enantio-enriched

\begin{tabular}{|c|c|c|c|}
\hline & Retention Time & Area & \%Area \\
\hline 1 & 35.511 & 53560697 & 89.021 \\
\hline 2 & 44.566 & 6605421 & 10.979 \\
\hline
\end{tabular}


Ethyl (R)-3-(2-(benzyloxy)-1-(((benzyloxy)carbonyl)amino)-2-oxoethyl)-4-oxo4H-chromene-2-carboxylate (6f)

$\begin{array}{ll}\mathrm{CO}_{2} \mathrm{Bn} & \begin{array}{l}\text { 5a at } 26^{\circ} \mathrm{C} \text { for } 27 \mathrm{~h} .57 \% \text { yield }(58.6 \mathrm{mg}), \text { colorless oil, } 89 \% \\ \text { ee. } \mathrm{HPLC}(\text { chiral IA column), hexane } / i-\mathrm{PrOH}=70 / 30, \text { flow } \\ \text { rate } 0.8 \mathrm{ml} / \mathrm{min}, \lambda=254 \mathrm{~nm}, t \mathrm{r} \text { (minor) }=36.29 \mathrm{~min}, t \mathrm{tr} \text { (major) }\end{array}\end{array}$ $=42.44 \mathrm{~min}$.

$[\alpha]^{25}=-23.4\left(\mathrm{c}=1.0\right.$ in $\left.\mathrm{CH}_{2} \mathrm{Cl}_{2}\right)$.

${ }^{1} \mathrm{H}$ NMR (400 MHz, $\left.\mathrm{CDCl}_{3}\right) \delta 8.15(\mathrm{~d}, J=8.0 \mathrm{~Hz}, 1 \mathrm{H}), 7.74(\mathrm{t}, J=7.8 \mathrm{~Hz}, 1 \mathrm{H}), 7.55$ $(\mathrm{d}, J=8.4 \mathrm{~Hz}, 1 \mathrm{H}), 7.44(\mathrm{t}, J=7.4 \mathrm{~Hz}, 1 \mathrm{H}), 7.32-7.25(\mathrm{~m}, 10 \mathrm{H}), 6.60(\mathrm{~d}, J=8.8 \mathrm{~Hz}$, $1 \mathrm{H}), 6.31(\mathrm{~d}, J=8.8 \mathrm{~Hz}, 1 \mathrm{H}), 5.24(\mathrm{~d}, J=12.6 \mathrm{~Hz}, 1 \mathrm{H}), 5.16(\mathrm{~d}, J=12.6 \mathrm{~Hz}, 1 \mathrm{H})$, $5.12(\mathrm{~d}, J=12.2 \mathrm{~Hz}, 1 \mathrm{H}), 5.04(\mathrm{~d}, J=12.2 \mathrm{~Hz}, 1 \mathrm{H}), 4.50(\mathrm{dd}, J=14.0,7.0 \mathrm{~Hz}, 2 \mathrm{H})$, $1.44(\mathrm{t}, J=7.0 \mathrm{~Hz}, 3 \mathrm{H}) .{ }^{13} \mathrm{C}\{1 \mathrm{H}\} \mathrm{NMR}\left(100 \mathrm{MHz}, \mathrm{CDCl}_{3}\right) \delta 177.8,169.6,160.9$, 156.0, 155.4, 151.3, 136.3, 135.5, 135.1, 128.5, 128.2, 128.1, 128.1, 128.0, 126.0, 125.9, 123.4, 123.2, 118.6, 67.6, 67.1, 63.5, 50.2, 14.1. HRMS (FTMS-ESI), m/z calcd for $\mathrm{C}_{29} \mathrm{H}_{25} \mathrm{NNaO}_{8}{ }^{+}\left([\mathrm{M}+\mathrm{Na}]^{+}\right)$538.1472, found 538.1482.

$\mathrm{mV}$

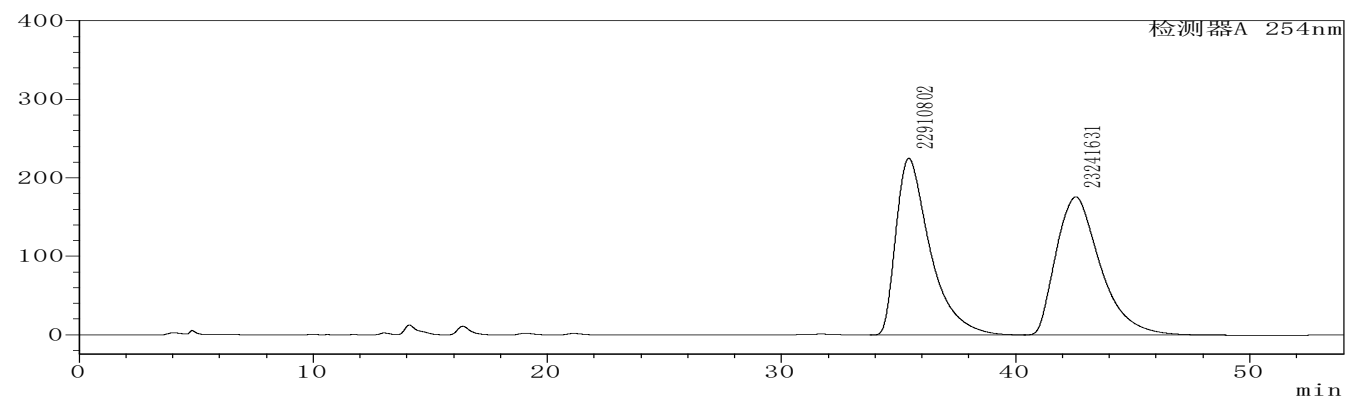

racemic

\begin{tabular}{|c|c|c|c|}
\hline & Retention Time & Area & \%Area \\
\hline 1 & 35.438 & 22910802 & 49.642 \\
\hline 2 & 42.571 & 23241631 & 50.358 \\
\hline
\end{tabular}

$\mathrm{mV}$

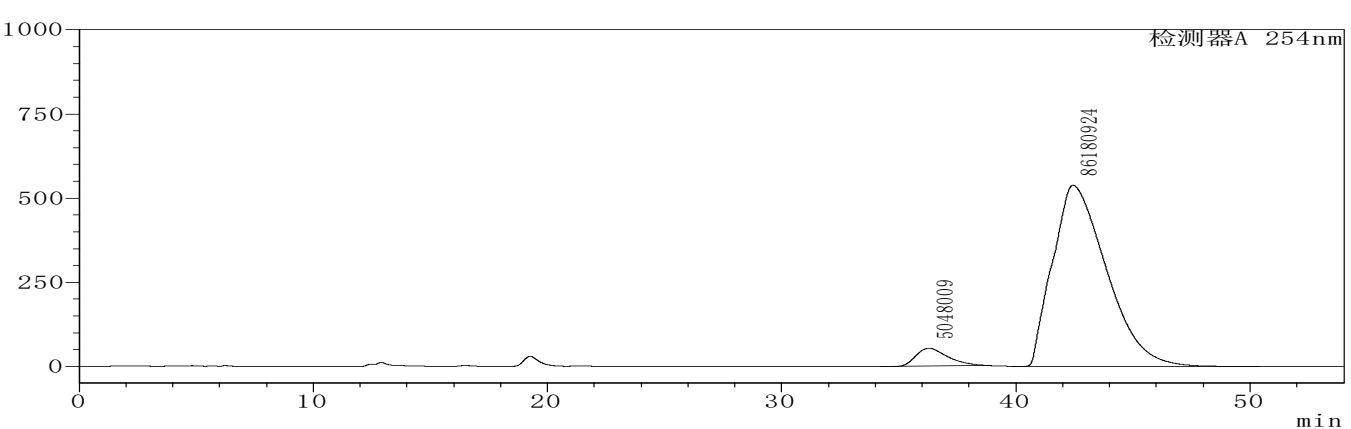

enantio-enriched

\begin{tabular}{|c|c|c|c|}
\hline & Retention Time & Area & \%Area \\
\hline 1 & 36.290 & 5048009 & 5.533 \\
\hline 2 & 42.441 & 86180924 & 94.467 \\
\hline
\end{tabular}


Benzyl (R)-2-(((benzyloxy)carbonyl)amino)-2-(4-0xo-2-phenyl-4H-chromen-3-yl) Acetate (6g)

$\mathrm{O} \quad \mathrm{NHCO}_{2} \mathrm{Bn}$ Compound 6g: Prepared in $0.2 \mathrm{mmol}$ scale using 1.2 equiv of<smiles>O=C(N[C@H](C(=O)OCc1ccccc1)c1c(-c2ccccc2)oc2ccccc2c1=O)c1ccccc1</smiles>
5a at $26^{\circ} \mathrm{C}$ for 27 h. $64 \%$ yield $(66.4 \mathrm{mg})$, colorless oil, $83 \%$ $e e$. HPLC (chiral IB column), hexane $/ i-\mathrm{PrOH}=80 / 20$, flow rate $0.8 \mathrm{ml} / \mathrm{min}, \lambda=254 \mathrm{~nm}, \operatorname{tr}$ (minor) $=15.59 \mathrm{~min}, \operatorname{tr}$ (major)

$=17.92 \mathrm{~min}$.

$[\alpha]^{25} \mathrm{D}=-58.3\left(\mathrm{c}=1.0\right.$ in $\left.\mathrm{CH}_{2} \mathrm{Cl}_{2}\right)$.

${ }^{1} \mathrm{H}$ NMR $\left(400 \mathrm{MHz}, \mathrm{CDCl}_{3}\right) \delta 8.21(\mathrm{~d}, J=8.0 \mathrm{~Hz}, 1 \mathrm{H}), 7.77(\mathrm{~d}, J=6.8 \mathrm{~Hz}, 2 \mathrm{H}), 7.71$ $(\mathrm{t}, J=7.8 \mathrm{~Hz}, 1 \mathrm{H}), 7.61-7.52(\mathrm{~m}, 3 \mathrm{H}), 7.52-7.48(\mathrm{~m}, 1 \mathrm{H}), 7.44(\mathrm{t}, J=7.6 \mathrm{~Hz}, 1 \mathrm{H})$, $7.38-7.27(\mathrm{~m}, 10 \mathrm{H}), 6.53(\mathrm{~d}, J=9.6 \mathrm{~Hz}, 1 \mathrm{H}), 5.60(\mathrm{~d}, J=9.6 \mathrm{~Hz}, 1 \mathrm{H}), 5.29(\mathrm{~d}, J=$ $12.4 \mathrm{~Hz}, 1 \mathrm{H}), 5.16(\mathrm{~d}, J=12.4 \mathrm{~Hz}, 1 \mathrm{H}), 5.12(\mathrm{~d}, J=12.4 \mathrm{~Hz}, 1 \mathrm{H}), 5.06(\mathrm{~d}, J=12.4$ $\mathrm{Hz}, 1 \mathrm{H}) .{ }^{13} \mathrm{C}\{1 \mathrm{H}\} \mathrm{NMR}\left(100 \mathrm{MHz}, \mathrm{CDCl}_{3}\right) \delta 178.1,170.2,164.6,156.3,156.1$, $136.3,135.5,134.3,131.8,131.2,129.1,128.9,128.5,128.5,128.2,128.0,128.0$, 127.9, 125.7, 125.4, 123.0, 118.9, 118.2, 67.6, 67.0, 52.8. HRMS (FTMS-ESI), m/z calcd for $\mathrm{C}_{32} \mathrm{H}_{26} \mathrm{NO}_{6}{ }^{+}\left([\mathrm{M}+\mathrm{H}]^{+}\right)$520.1755, found 520.1763 .

$\mathrm{mV}$

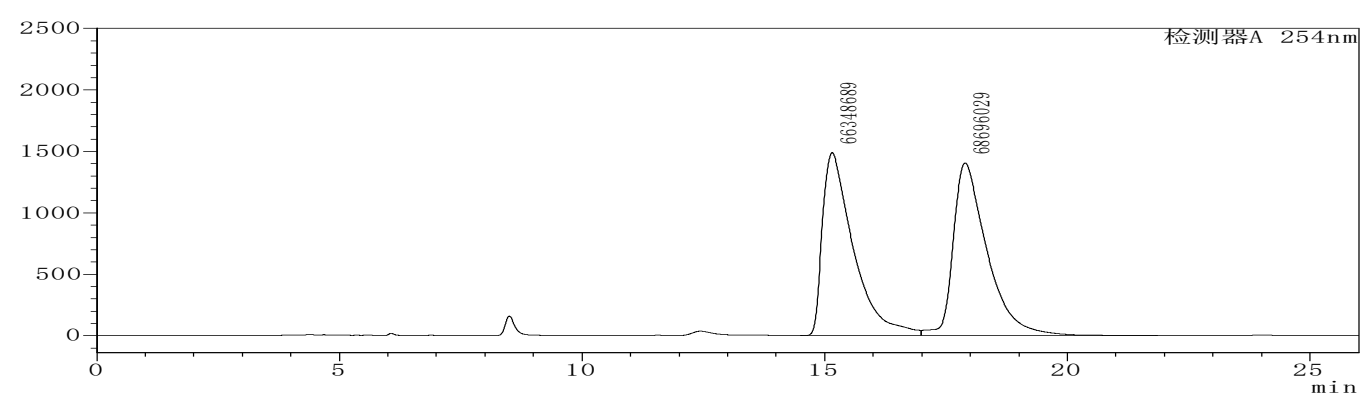

racemic

\begin{tabular}{|c|c|c|c|}
\hline & Retention Time & Area & \%Area \\
\hline 1 & 15.152 & 66348689 & 49.131 \\
\hline 2 & 17.894 & 68696029 & 50.869 \\
\hline
\end{tabular}

$\mathrm{mV}$

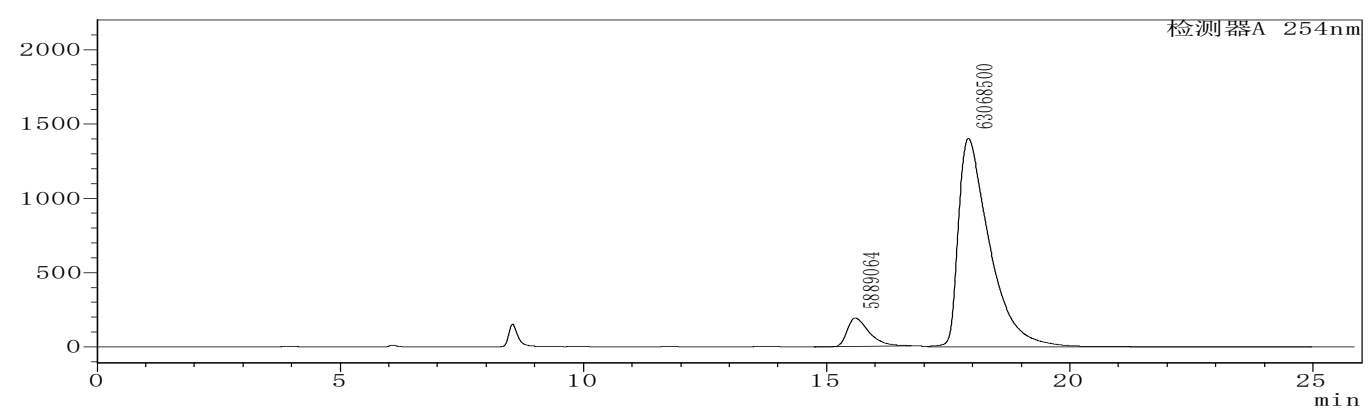

enantio-enriched

\begin{tabular}{|c|c|c|c|}
\hline & Retention Time & Area & \%Area \\
\hline 1 & 15.591 & 5889064 & 8.540 \\
\hline 2 & 17.915 & 63068500 & 91.460 \\
\hline
\end{tabular}


Benzyl $(R)-2-(((b e n z y l o x y)$ carbonyl)amino)-2-(6-chloro-2-methyl-4-oxo-4H-chromen-3-yl)acetate $(6 \mathrm{~h})$<smiles>Cc1oc2ccc(Cl)cc2c(=O)c1[C@H](NC(=O)OCc1ccccc1)C(=O)OCc1ccccc1</smiles>

Compound 6h: Prepared in $0.2 \mathrm{mmol}$ scale using 1.2 equiv of $5 \mathbf{a}$ at $26^{\circ} \mathrm{C}$ for 27 h. $60 \%$ yield $(59.5 \mathrm{mg})$, white solid; mp: $118.7-120.2{ }^{\circ} \mathrm{C}, 88 \%$ ee. HPLC (chiral IB column), hexane $/ i-\mathrm{PrOH}=80 / 20$, flow rate $0.8 \mathrm{ml} / \mathrm{min}, \lambda$ $=254 \mathrm{~nm}, t \mathrm{r}($ minor $)=13.16 \mathrm{~min}, t \mathrm{r}($ major $)=14.55 \mathrm{~min}$.

$[\alpha]^{25} \mathrm{D}=-58.8\left(\mathrm{c}=1.0\right.$ in $\left.\mathrm{CH}_{2} \mathrm{Cl}_{2}\right)$.

${ }^{1} \mathrm{H} \mathrm{NMR}\left(400 \mathrm{MHz}, \mathrm{CDCl}_{3}\right) \delta 8.07(\mathrm{~d}, J=2.4 \mathrm{~Hz}, 1 \mathrm{H}), 7.59(\mathrm{dd}, J=9.0,2.4 \mathrm{~Hz}, 1 \mathrm{H})$, $7.38(\mathrm{~d}, J=9.0 \mathrm{~Hz}, 1 \mathrm{H}), 7.35-7.22(\mathrm{~m}, 10 \mathrm{H}), 6.42(\mathrm{~d}, J=8.8 \mathrm{~Hz}, 1 \mathrm{H}), 5.44(\mathrm{~d}, J=$ $8.8 \mathrm{~Hz}, 1 \mathrm{H}), 5.23(\mathrm{~d}, J=12.4 \mathrm{~Hz}, 1 \mathrm{H}), 5.15(\mathrm{~d}, J=6.4 \mathrm{~Hz}, 1 \mathrm{H}), 5.12(\mathrm{~d}, J=6.4 \mathrm{~Hz}$, $1 \mathrm{H}), 5.06(\mathrm{~d}, J=12.4 \mathrm{~Hz}, 1 \mathrm{H}), 2.66(\mathrm{~s}, 3 \mathrm{H}) .{ }^{13} \mathrm{C}\{1 \mathrm{H}\} \mathrm{NMR}\left(100 \mathrm{MHz}, \mathrm{CDCl}_{3}\right) \delta$ $176.1,169.7,165.7,156.3,154.5,136.2,135.4,134.2,131.2,128.6,128.4,128.2$, 128.0, 125.1, 124.0, 119.7, 119.4, 67.8, 67.2, 51.9, 18.7. HRMS (FTMS-ESI), m/z calcd for $\mathrm{C}_{27} \mathrm{H}_{23} \mathrm{ClNO}_{6}{ }^{+}\left([\mathrm{M}+\mathrm{H}]^{+}\right) 492.1208$ (494.1179 for $\left.{ }^{37} \mathrm{Cl}\right)$, found 492.1208 $\left(494.1188\right.$ for $\left.{ }^{37} \mathrm{Cl}\right)$.

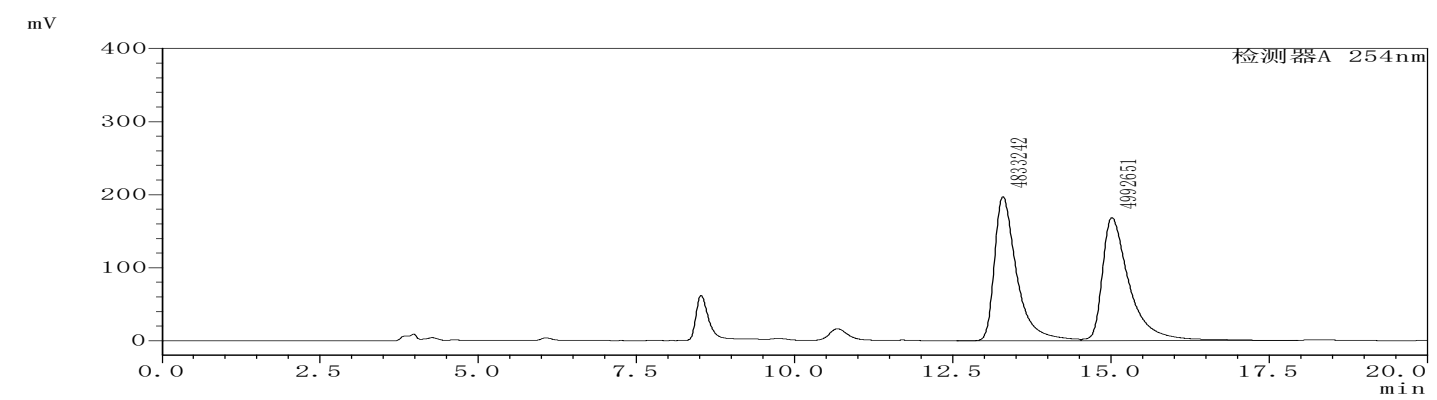

racemic

\begin{tabular}{|c|c|c|c|}
\hline & Retention Time & Area & \%Area \\
\hline 1 & 13.294 & 4833242 & 49.189 \\
\hline 2 & 15.018 & 4992651 & 50.811 \\
\hline
\end{tabular}

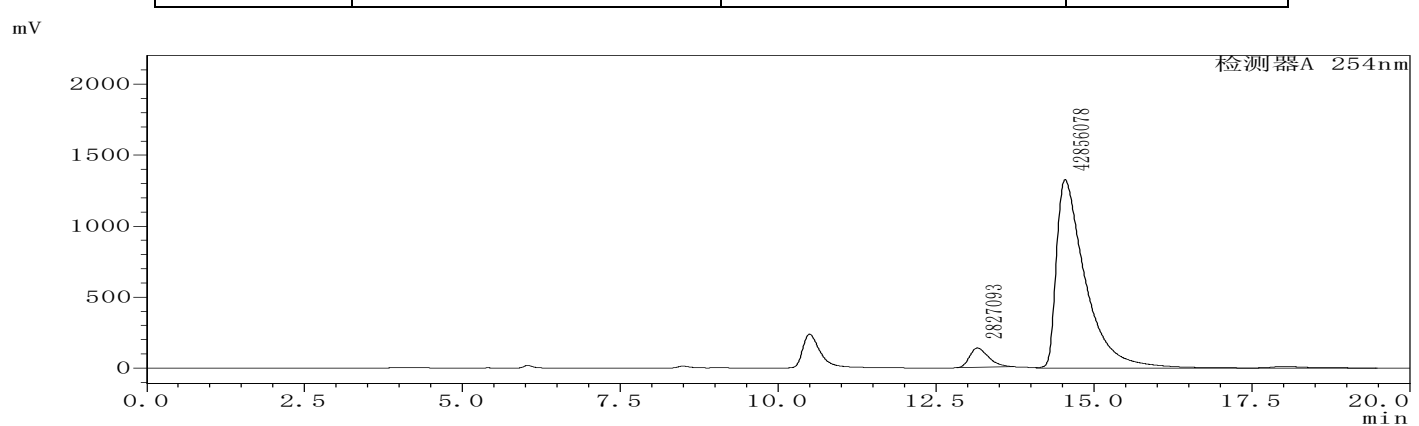

enantio-enriched

\begin{tabular}{|c|c|c|c|}
\hline & Retention Time & Area & \%Area \\
\hline 1 & 13.156 & 2827093 & 6.188 \\
\hline 2 & 14.545 & 42856078 & 93.812 \\
\hline
\end{tabular}


Benzyl (R)-2-(((benzyloxy)carbonyl)amino)-2-(2,6-dimethyl-4-oxo-4H-chromen3-yl)acetate (6i)<smiles>Cc1ccc2oc(C)c([C@H](NC(=O)c3ccccc3)C(=O)O)c(=O)c2c1</smiles>

Compound 6i: Prepared in $0.2 \mathrm{mmol}$ scale using 1.2 equiv of $\mathbf{5 a}$ at $26^{\circ} \mathrm{C}$ for 27 h. $41 \%$ yield $(38.7 \mathrm{mg})$, colorless oil, $75 \%$ ee. HPLC (chiral IB column), hexane $/ i-\mathrm{PrOH}=80 / 20$, flow rate $0.8 \mathrm{ml} / \mathrm{min}, \lambda=254 \mathrm{~nm}, t \mathrm{r}($ minor $)=11.65 \mathrm{~min}$,

$\operatorname{tr}($ major $)=13.00 \mathrm{~min}$.

$[\alpha]^{25} \mathrm{D}=-41.4\left(\mathrm{c}=1.0\right.$ in $\left.\mathrm{CH}_{2} \mathrm{Cl}_{2}\right)$.

${ }^{1} \mathrm{H}$ NMR $\left(400 \mathrm{MHz}, \mathrm{CDCl}_{3}\right) \delta 7.90(\mathrm{~s}, 1 \mathrm{H}), 7.48-7.44(\mathrm{~m}, 1 \mathrm{H}), 7.35-7.23(\mathrm{~m}, 11 \mathrm{H})$, $6.47(\mathrm{~d}, J=9.0 \mathrm{~Hz}, 1 \mathrm{H}), 5.44(\mathrm{~d}, J=9.0 \mathrm{~Hz}, 1 \mathrm{H}), 5.24(\mathrm{~d}, J=12.4 \mathrm{~Hz}, 1 \mathrm{H}), 5.13(\mathrm{~d}, J$ $=12.4 \mathrm{~Hz}, 2 \mathrm{H}), 5.05(\mathrm{~d}, J=12.4 \mathrm{~Hz}, 1 \mathrm{H}), 2.64(\mathrm{~s}, 3 \mathrm{H}), 2.44(\mathrm{~s}, 3 \mathrm{H}) .{ }^{13} \mathrm{C}\{1 \mathrm{H}\} \mathrm{NMR}$ $\left(100 \mathrm{MHz} \mathrm{CDCl}_{3}\right) \delta 177.3,170.0,165.2,156.4,154.5,136.3,135.6,135.2,128.6$, 128.3, 128.2, 128.0, 125.0, 122.8, 119.1, 117.7, 110.2, 67.7, 67.1, 52.1, 21.1, 18.7. HRMS (FTMS-ESI), m/z calcd for $\mathrm{C}_{28} \mathrm{H}_{25} \mathrm{NNaO}_{6}{ }^{+}\left([\mathrm{M}+\mathrm{Na}]^{+}\right) 494.1574$, found 494.1576.

$\mathrm{mV}$

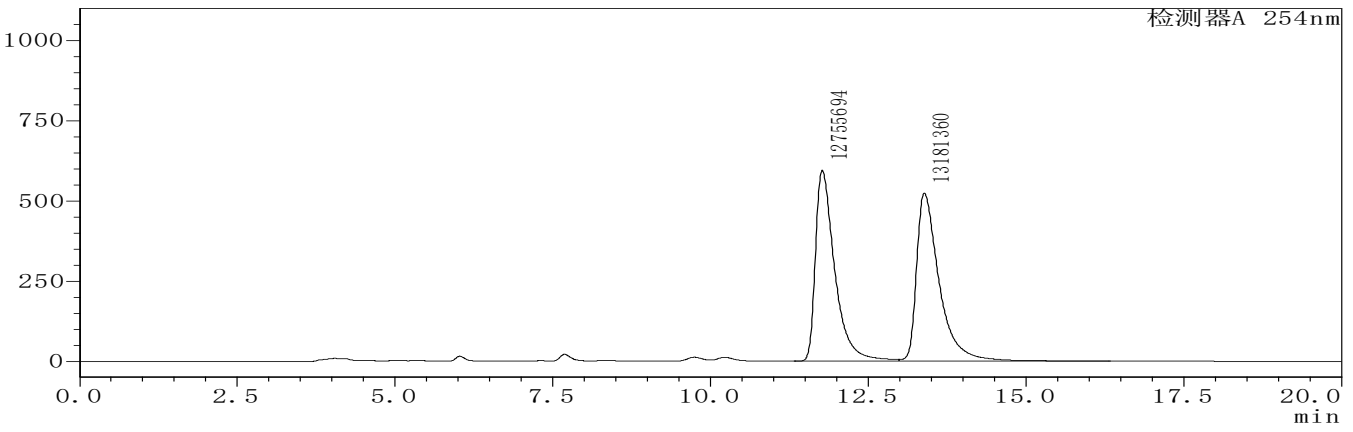

racemic

\begin{tabular}{|c|c|c|c|}
\hline & Retention Time & Area & \%Area \\
\hline 1 & 11.772 & 12755694 & 49.179 \\
\hline 2 & 13.391 & 13181360 & 50.821 \\
\hline
\end{tabular}

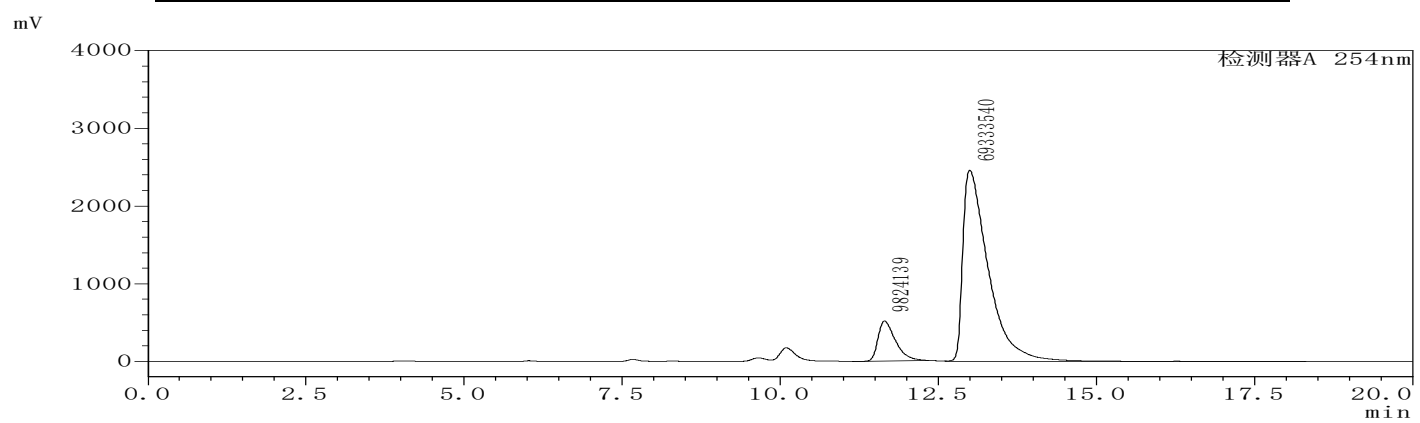

enantio-enriched

\begin{tabular}{|c|c|c|c|}
\hline & Retention Time & Area & \%Area \\
\hline 1 & 11.650 & 9824139 & 12.411 \\
\hline 2 & 12.998 & 69333540 & 87.589 \\
\hline
\end{tabular}


Benzyl (R)-2-amino-2-(2-methyl-4-oxo-4H-chromen-3-yl)acetate (6k)<smiles>Cc1oc2ccccc2c(=O)c1[C@H](N)C(=O)OCc1ccccc1</smiles>

Compound 6k: Prepared in $0.2 \mathrm{mmol}$ scale using 1.2 equiv of 5a at $26{ }^{\circ} \mathrm{C}$ for 27 h. $24 \%$ yield $(15.3 \mathrm{mg})$, colorless oil, $75 \%$ $e e$. HPLC (chiral IA column), hexane $/ i-\mathrm{PrOH}=70 / 30$, flow rate $0.8 \mathrm{ml} / \mathrm{min}, \lambda=254 \mathrm{~nm}, \operatorname{tr}$ (minor) $=12.90 \mathrm{~min}, \operatorname{tr}$ (major)

$=17.24 \mathrm{~min}$.

$[\alpha]^{25} \mathrm{D}=-2.0\left(\mathrm{c}=1.0\right.$ in $\left.\mathrm{CH}_{2} \mathrm{Cl}_{2}\right)$.

${ }^{1} \mathrm{H}$ NMR $\left(400 \mathrm{MHz}, \mathrm{CDCl}_{3}\right) \delta 8.13(\mathrm{~d}, J=7.6 \mathrm{~Hz}, 1 \mathrm{H}), 7.63(\mathrm{t}, J=7.4 \mathrm{~Hz}, 1 \mathrm{H}), 7.41$ $-7.32(\mathrm{~m}, 2 \mathrm{H}), 7.31-7.17(\mathrm{~m}, 5 \mathrm{H}), 5.16(\mathrm{q}, J=12.2 \mathrm{~Hz}, 2 \mathrm{H}), 4.50(\mathrm{~s}, 1 \mathrm{H}), 2.48$ (s, $3 \mathrm{H}), 2.31(\mathrm{~s}, 2 \mathrm{H}) .{ }^{13} \mathrm{C}\{1 \mathrm{H}\} \mathrm{NMR}\left(100 \mathrm{MHz}, \mathrm{CDCl}_{3}\right) \delta 177.0,173.8,164.6,156.0$, 135.9, 133.7, 128.5, 128.3, 128.2, 125.9, 125.1, 123.2, 121.1, 117.8, 67.3, 52.4, 18.6. HRMS (FTMS-ESI), m/z calcd for $\mathrm{C}_{19} \mathrm{H}_{17} \mathrm{NNaO}_{4}{ }^{+}\left([\mathrm{M}+\mathrm{Na}]^{+}\right) 346.1050$, found 346.1047 .

$\mathrm{mV}$

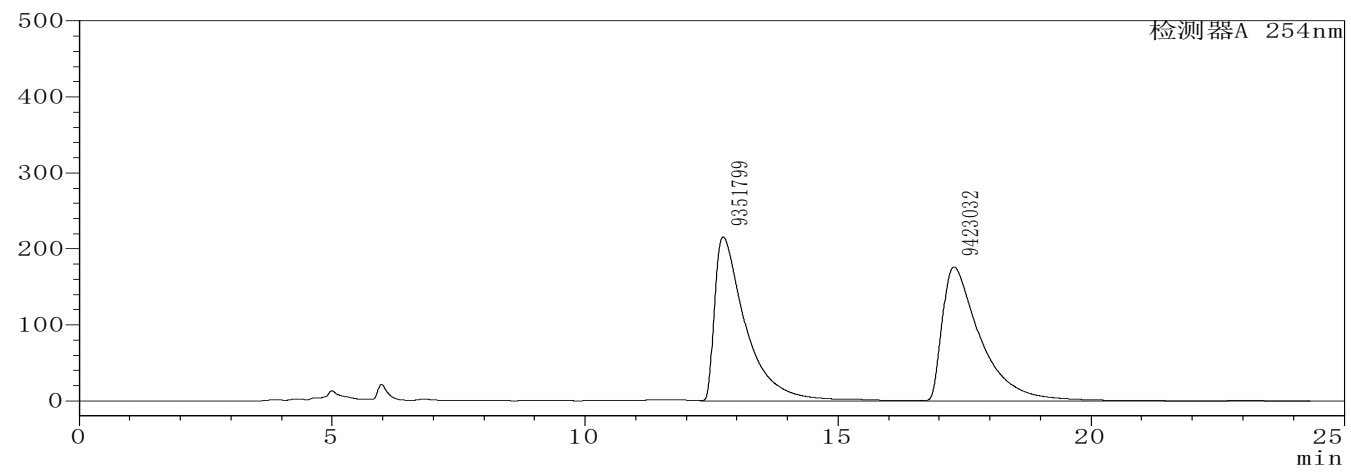

racemic

\begin{tabular}{|c|c|c|c|}
\hline & Retention Time & Area & \%Area \\
\hline 1 & 12.728 & 9351799 & 49.810 \\
\hline 2 & 17.289 & 9423032 & 50.190 \\
\hline
\end{tabular}

$\mathrm{mV}$

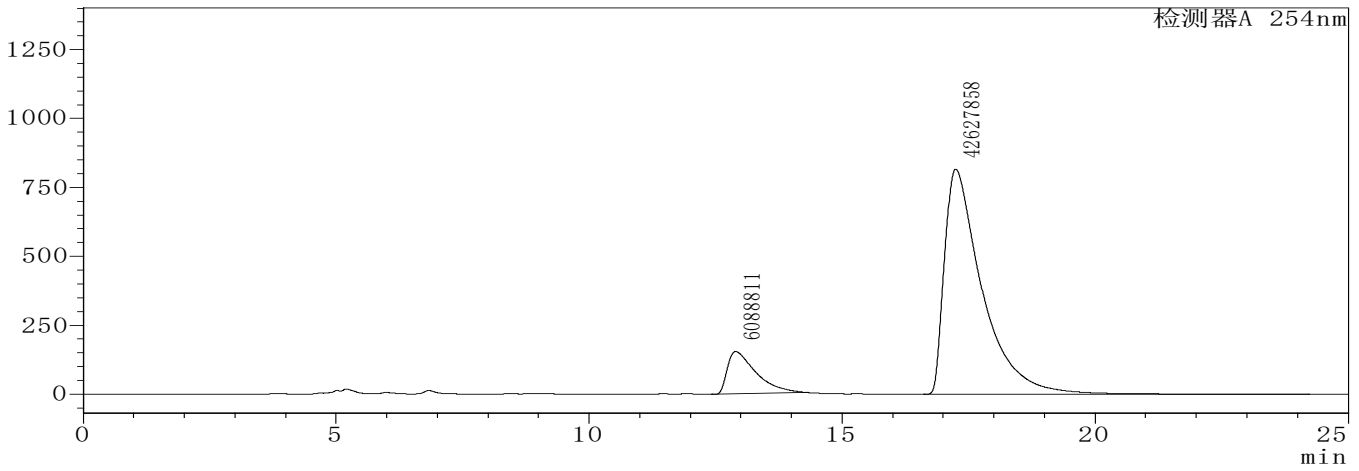

enantio-enriched

\begin{tabular}{|c|c|c|c|}
\hline & Retention Time & Area & \%Area \\
\hline 1 & 12.899 & 6088811 & 12.498 \\
\hline 2 & 17.244 & 42627858 & 87.502 \\
\hline
\end{tabular}




\section{Compound (7a)}<smiles>Cc1oc2ccccc2c(=O)c1C(C#Cc1ccccc1)NC(NC(=O)OC(C)(C)C)=[N+](C)C(C)(C)C</smiles>

Compound 7a: Prepared in $0.2 \mathrm{mmol} \mathrm{scale}$ at $50{ }^{\circ} \mathrm{C}$ for $27 \mathrm{~h}$. $68 \%$ yield $(72.3 \mathrm{mg}$ ), yellow oil, 94\% ee. HPLC (chiral IC column), hexane $/ i-\mathrm{PrOH}=70 / 30$, flow rate $0.8 \mathrm{ml} / \mathrm{min}, \lambda=$ $254 \mathrm{~nm}, t \mathrm{r}$ (major) $=5.80 \mathrm{~min}, \mathrm{tr}($ minor $)=12.32 \mathrm{~min}$. $[\alpha]^{25} \mathrm{D}=-16.4\left(\mathrm{c}=1.0\right.$ in $\left.\mathrm{CH}_{2} \mathrm{Cl}_{2}\right)$.

${ }^{1} \mathrm{H}$ NMR $\left(400 \mathrm{MHz}, \mathrm{CDCl}_{3}\right) \delta 11.27(\mathrm{~s}, 1 \mathrm{H}), 9.60(\mathrm{~d}, J=8.4$ $\mathrm{Hz}, 1 \mathrm{H}), 8.24(\mathrm{~d}, J=8.0 \mathrm{~Hz}, 1 \mathrm{H}), 7.62(\mathrm{t}, J=7.8 \mathrm{~Hz}, 1 \mathrm{H}), 7.44-7.31(\mathrm{~m}, 4 \mathrm{H}), 7.27-$ $7.21(\mathrm{~m}, 3 \mathrm{H}), 6.53(\mathrm{~d}, J=8.4 \mathrm{~Hz}, 1 \mathrm{H}), 2.75(\mathrm{~s}, 3 \mathrm{H}), 1.49(\mathrm{~s}, 18 \mathrm{H}) .{ }^{13} \mathrm{C}\{1 \mathrm{H}\} \mathrm{NMR}$ $\left(100 \mathrm{MHz} \mathrm{CDCl}_{3}\right) \delta 176.4,164.1,163.4,156.0,155.0,152.3,133.53,132.1,128.3$, $128.2,126.1,125.0,123.8,122.9,119.2,117.7,86.3,83.2,82.6,79.0,40.5,28.4,28.2$, 18.7. HRMS (TOFMS-ESI), $\mathrm{m} / \mathrm{z}$ calcd for $\mathrm{C}_{30} \mathrm{H}_{34} \mathrm{~N}_{3} \mathrm{O}_{6}{ }^{+}\left([\mathrm{M}+\mathrm{H}]^{+}\right) 532.2442$, found 532.2451 .

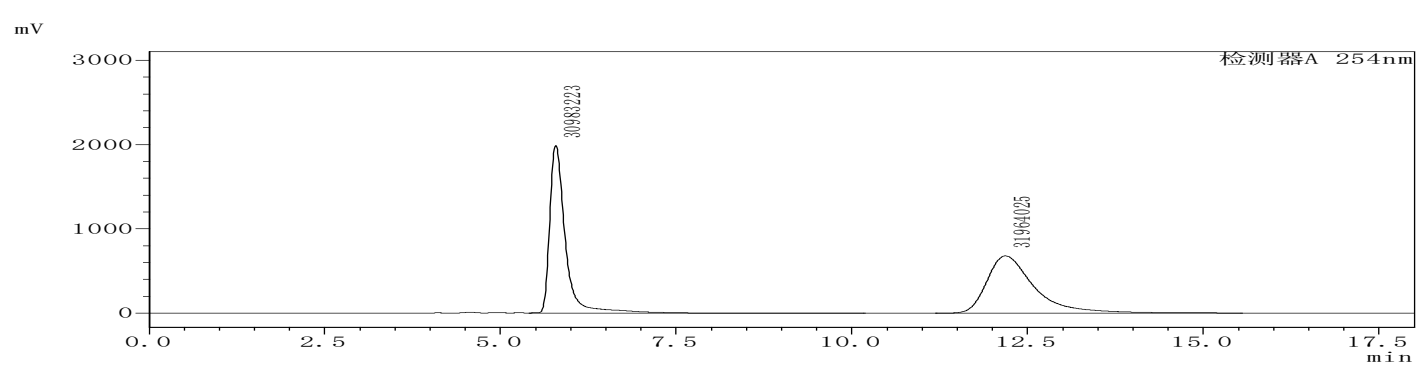

racemic

\begin{tabular}{|c|c|c|c|}
\hline & Retention Time & Area & \%Area \\
\hline 1 & 5.787 & 30983223 & 49.221 \\
\hline 2 & 12.185 & 31964025 & 50.779 \\
\hline
\end{tabular}

$\mathrm{mV}$

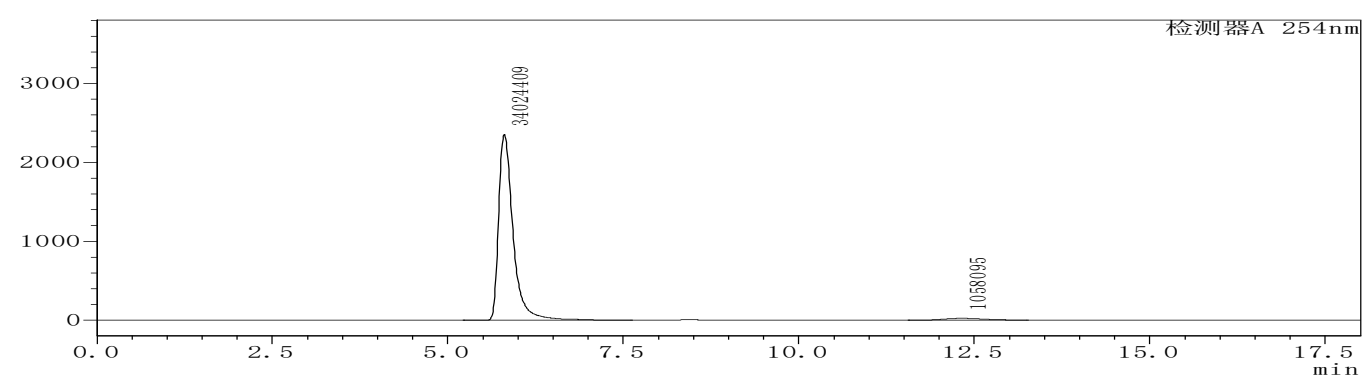

enantio-enriched

\begin{tabular}{|c|c|c|c|}
\hline & Retention Time & Area & \%Area \\
\hline 1 & 5.804 & 34024409 & 96.984 \\
\hline 2 & 12.322 & 1058095 & 3.016 \\
\hline
\end{tabular}

(S)-4-Methyl-N-(1-(2-methyl-4-oxo-4H-chromen-3-yl)-3-phenylprop-2-yn-1-yl)be -nzenesulfonamide (8a)<smiles>Cc1oc2ccccc2c(=O)c1[C@H]([NH3+])C#Cc1ccccc1</smiles>

Compound 8a: Prepared in $0.1 \mathrm{mmol}$ scale at $26{ }^{\circ} \mathrm{C}$ for $2.5 \mathrm{~h}$. $65 \%$ yield $(29.0 \mathrm{mg})$, white solid; $\mathrm{mp}: 174.2-175.7{ }^{\circ} \mathrm{C}, 95 \%$ $e e$. HPLC (chiral IA column), hexane $/ i-\mathrm{PrOH}=80 / 20$, flow rate $0.8 \mathrm{ml} / \mathrm{min}, \lambda=254 \mathrm{~nm}$, $t \mathrm{r}$ (minor) $=25.53 \mathrm{~min}, t \mathrm{r}$ $($ major $)=29.66 \mathrm{~min}$. 
$[\alpha]^{25} \mathrm{D}=+15.6\left(\mathrm{c}=1.0\right.$ in $\left.\mathrm{CH}_{2} \mathrm{Cl}_{2}\right)$.

${ }^{1} \mathrm{H}$ NMR $\left(400 \mathrm{MHz}, \mathrm{CDCl}_{3}\right) \delta 8.07(\mathrm{~d}, J=8.0 \mathrm{~Hz}, 1 \mathrm{H}), 7.71(\mathrm{~d}, J=8.0 \mathrm{~Hz}, 2 \mathrm{H}), 7.63$ $(\mathrm{t}, J=7.8 \mathrm{~Hz}, 1 \mathrm{H}), 7.40-7.31(\mathrm{~m}, 2 \mathrm{H}), 7.25-7.14(\mathrm{~m}, 5 \mathrm{H}), 7.03(\mathrm{~d}, J=8.0 \mathrm{~Hz}, 2 \mathrm{H})$, $6.81(\mathrm{~d}, J=9.6 \mathrm{~Hz}, 1 \mathrm{H}), 5.50(\mathrm{~d}, J=9.6 \mathrm{~Hz}, 1 \mathrm{H}), 2.54(\mathrm{~s}, 3 \mathrm{H}), 2.10(\mathrm{~s}, 3 \mathrm{H}) .{ }^{13} \mathrm{C}\{1 \mathrm{H}\}$ NMR $\left(100 \mathrm{MHz}, \mathrm{CDCl}_{3}\right) \delta 176.5,163.4,155.7,143.4,137.7,133.9,131.8,129.3$, 128.5, 128.1, 127.2, 125.7, 125.2, 123.2, 122.2, 118.1, 117.7, 84.8, 83.9, 44.5, 21.3, 18.5. HRMS (FTMS-ESI), m/z calcd for $\mathrm{C}_{26} \mathrm{H}_{22} \mathrm{NO}_{4} \mathrm{~S}^{+}\left([\mathrm{M}+\mathrm{H}]^{+}\right) 444.1264$, found 444.1260.

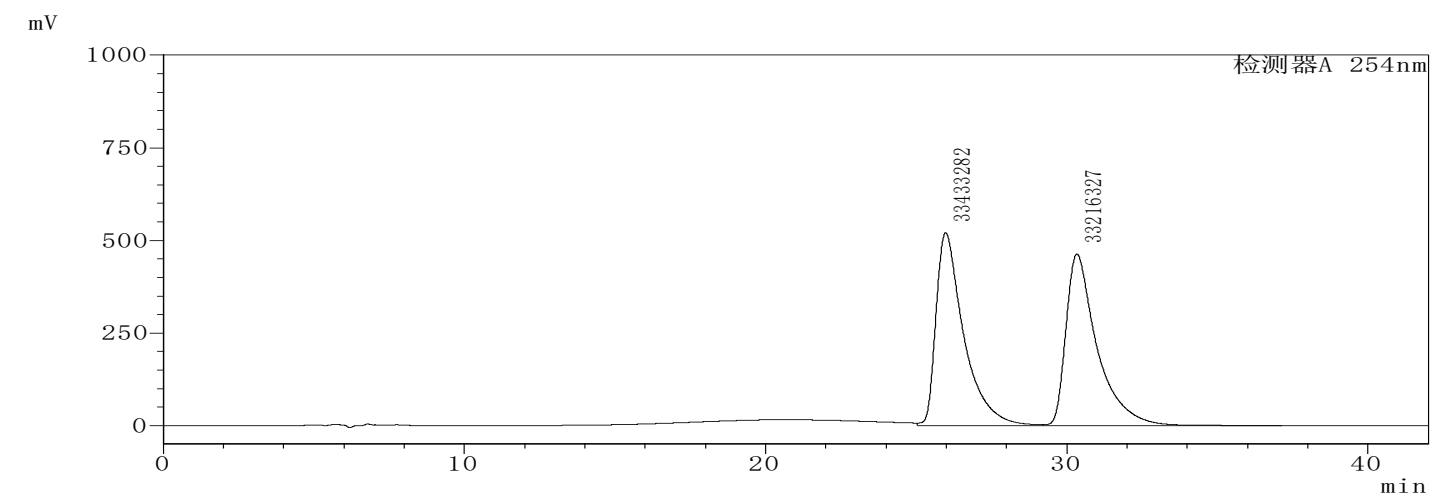

racemic

\begin{tabular}{|c|c|c|c|}
\hline & Retention Time & Area & \%Area \\
\hline 1 & 25.981 & 33433282 & 50.163 \\
\hline 2 & 30.342 & 33216327 & 49.837 \\
\hline
\end{tabular}

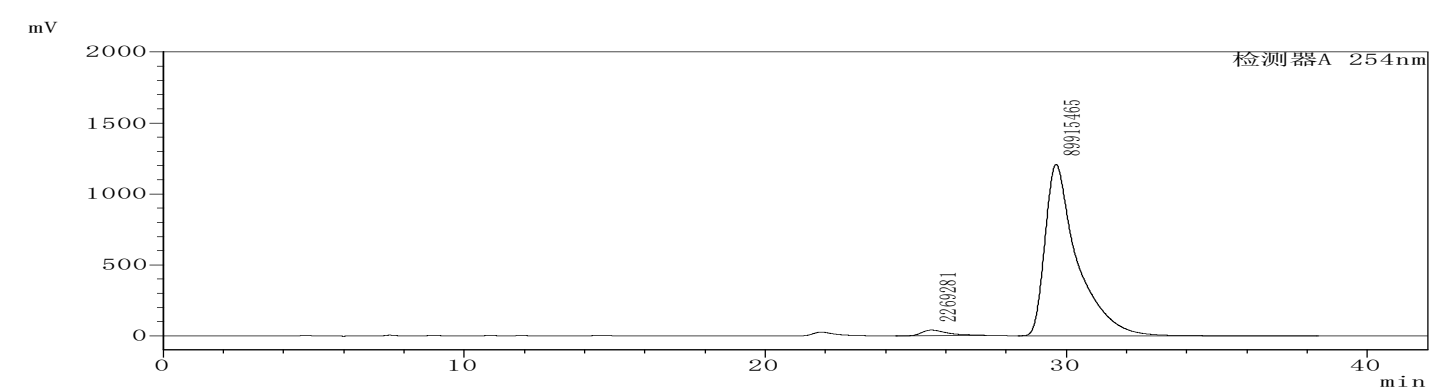

enantio-enriched

\begin{tabular}{|c|c|c|c|}
\hline & Retention Time & Area & \%Area \\
\hline 1 & 25.528 & 2269281 & 2.462 \\
\hline 2 & 29.664 & 89915465 & 97.538 \\
\hline
\end{tabular}

tert-Butyl (S)-(1-(2-methyl-4-oxo-4H-chromen-3-yl)prop-2-yn-1-yl)carbamate

(9a)

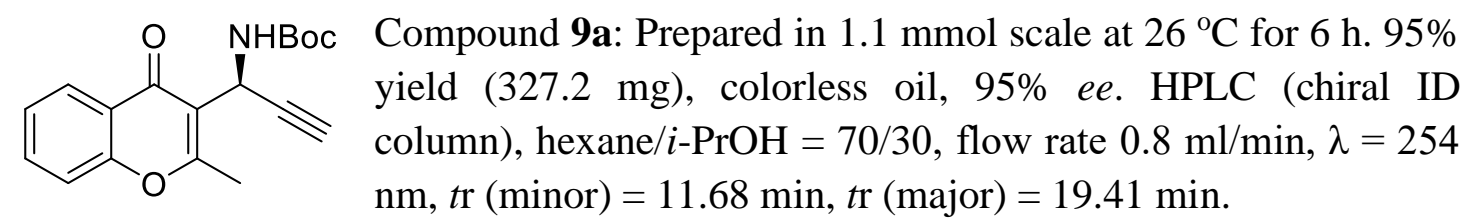
$[\alpha]^{25}{ }_{\mathrm{D}}=-25.1\left(\mathrm{c}=1.0\right.$ in $\left.\mathrm{CH}_{2} \mathrm{Cl}_{2}\right)$.

${ }^{1} \mathrm{H}$ NMR $\left(400 \mathrm{MHz}, \mathrm{CDCl}_{3}\right) \delta 8.14(\mathrm{~d}, J=7.6 \mathrm{~Hz}, 1 \mathrm{H}), 7.66-7.50(\mathrm{~m}, 1 \mathrm{H}), 7.41-$ $7.31(\mathrm{~m}, 2 \mathrm{H}), 6.41(\mathrm{~d}, J=8.8 \mathrm{~Hz}, 1 \mathrm{H}), 5.92-5.41(\mathrm{~m}, 1 \mathrm{H}), 2.58(\mathrm{~s}, 3 \mathrm{H}), 2.30(\mathrm{~s}, 1 \mathrm{H})$, 
$1.38(\mathrm{~s}, 9 \mathrm{H}) .{ }^{13} \mathrm{C}\{1 \mathrm{H}\} \mathrm{NMR}\left(150 \mathrm{MHz}, \mathrm{CDCl}_{3}\right) \delta 177.0,163.6,155.9,155.0,133.8$, $125.7,125.1,123.4,119.1,117.8,81.4,80.0,70.3,40.2,28.4$, 18.4. HRMS (FTMS-ESI), m/z calcd for $\mathrm{C}_{18} \mathrm{H}_{19} \mathrm{NNaO}_{4}{ }^{+}\left([\mathrm{M}+\mathrm{Na}]^{+}\right) 336.1206$, found 336.1204.

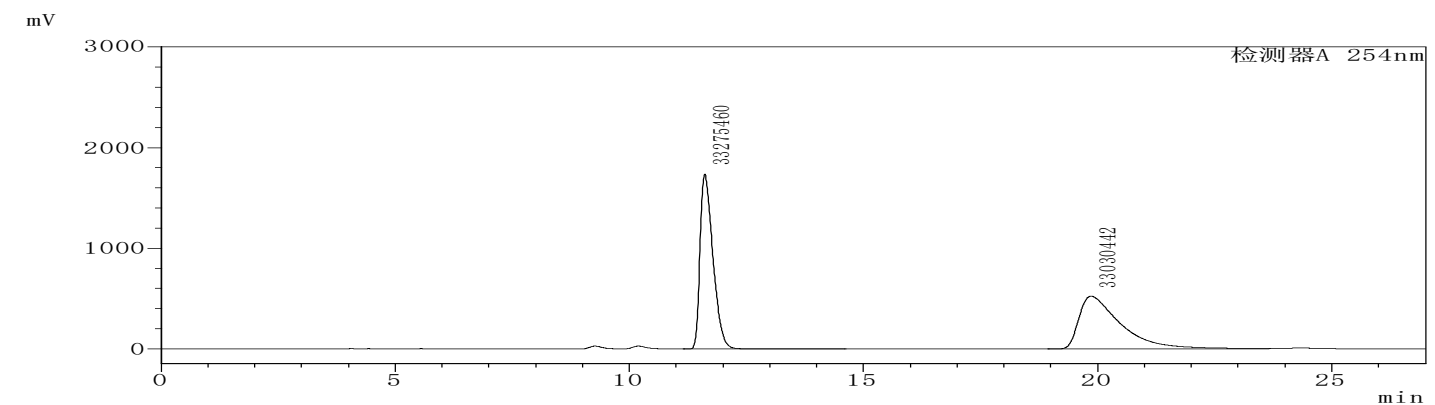

racemic

\begin{tabular}{|c|c|c|c|}
\hline & Retention Time & Area & \%Area \\
\hline 1 & 11.611 & 33275460 & 50.185 \\
\hline 2 & 19.863 & 33030442 & 49.815 \\
\hline
\end{tabular}

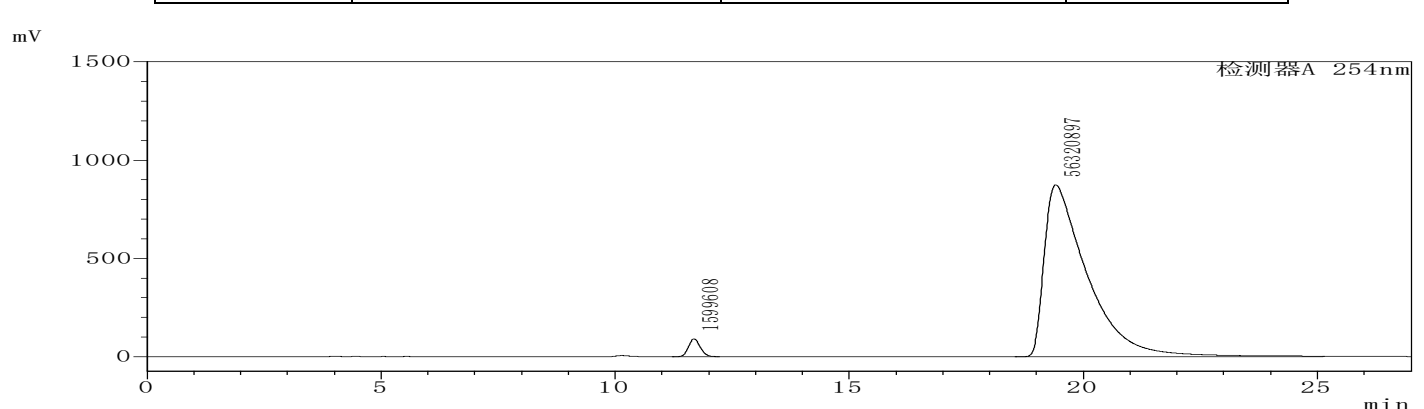

enantio-enriched

\begin{tabular}{|c|c|c|c|}
\hline & Retention Time & Area & \%Area \\
\hline 1 & 11.684 & 1497860 & 2.591 \\
\hline 2 & 19.411 & 56320897 & 97.409 \\
\hline
\end{tabular}

tert-Butyl (R)-((2-methyl-4-oxo-4H-chromen-3-yl)(1-tosyl-1H-1,2,3-triazol-4-yl) methyl)carbamate $(9 \mathrm{~b})$<smiles>Cc1oc2ccccc2c(=O)c1[C@H](N)C(=O)OC(C)(C)C</smiles>
Compound 9b: Prepared in $0.1 \mathrm{mmol}$ scale at $0{ }^{\circ} \mathrm{C}$ for $1 \mathrm{~h}$. 99\% yield (55.4 mg), white solid; mp: $186.3-187.1{ }^{\circ} \mathrm{C}, 95 \%$ ee. HPLC (chiral IB column), hexane $/ i-\mathrm{PrOH}=90 / 10$, flow rate $0.8 \mathrm{ml} / \mathrm{min}, \lambda=254 \mathrm{~nm}, t \mathrm{r}$ (major) $=14.53 \mathrm{~min}$,

$\operatorname{tr}($ minor $)=19.86 \mathrm{~min}$. $[\alpha]^{25} \mathrm{D}=-9.7\left(\mathrm{c}=1.0\right.$ in $\left.\mathrm{CH}_{2} \mathrm{Cl}_{2}\right)$.

${ }^{1} \mathrm{H}$ NMR (400 MHz, $\left.\mathrm{CDCl}_{3}\right) \delta 8.20(\mathrm{~s}, 1 \mathrm{H}), 8.03(\mathrm{~d}, J=8.0 \mathrm{~Hz}, 1 \mathrm{H}), 7.95(\mathrm{~d}, J=8.2$ $\mathrm{Hz}, 2 \mathrm{H}), 7.62(\mathrm{t}, J=7.8 \mathrm{~Hz}, 1 \mathrm{H}), 7.38(\mathrm{~d}, J=8.4 \mathrm{~Hz}, 1 \mathrm{H}), 7.33(\mathrm{~d}, J=8.0 \mathrm{~Hz}, 3 \mathrm{H})$, $6.80(\mathrm{~d}, J=9.8 \mathrm{~Hz}, 1 \mathrm{H}), 6.14(\mathrm{~d}, J=9.8 \mathrm{~Hz}, 1 \mathrm{H}), 2.70(\mathrm{~s}, 3 \mathrm{H}), 2.40(\mathrm{~s}, 3 \mathrm{H}), 1.41$ (s, 9H). ${ }^{13} \mathrm{C}\{1 \mathrm{H}\} \mathrm{NMR}\left(100 \mathrm{MHz}, \mathrm{CDCl}_{3}\right) \delta 177.6,164.9,156.0,155.4,148.5,147.3$, 133.9, 133.0, 130.4, 128.8, 125.4, 125.2, 123.3, 121.5, 119.1, 117.9, 80.1, 46.2, 28.4, 21.9, 18.9. HRMS (FTMS-ESI), $\mathrm{m} / \mathrm{z}$ calcd for $\mathrm{C}_{25} \mathrm{H}_{26} \mathrm{~N}_{4} \mathrm{NaO}_{6} \mathrm{~S}^{+}\left([\mathrm{M}+\mathrm{Na}]^{+}\right)$ 533.1465, found 533.1469. 


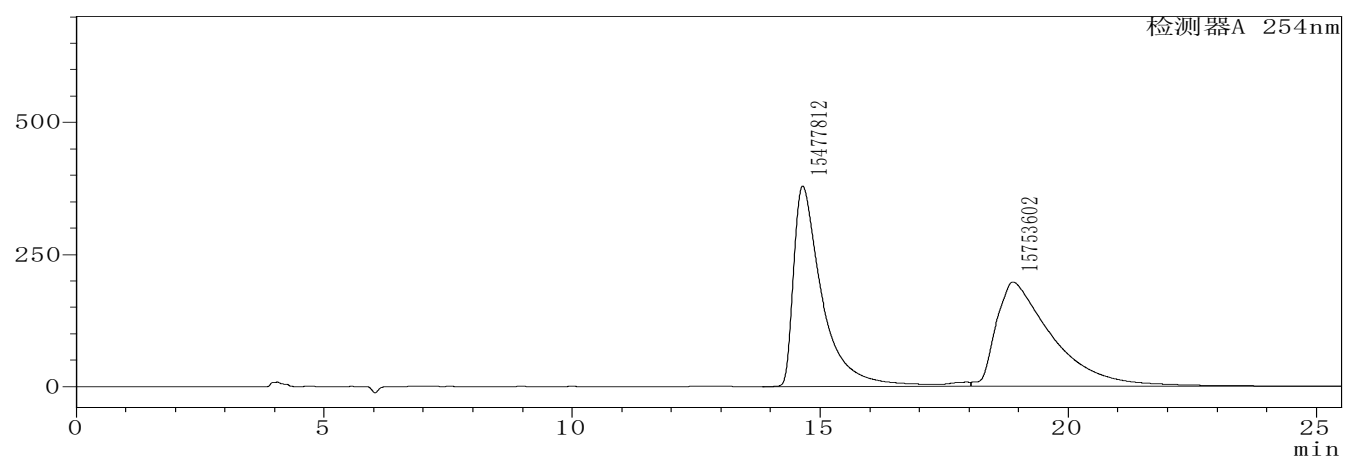

racemic

\begin{tabular}{|c|c|c|c|}
\hline & Retention Time & Area & \%Area \\
\hline 1 & 14.645 & 15477812 & 49.558 \\
\hline 2 & 18.888 & 15753602 & 50.442 \\
\hline
\end{tabular}

$\mathrm{mV}$

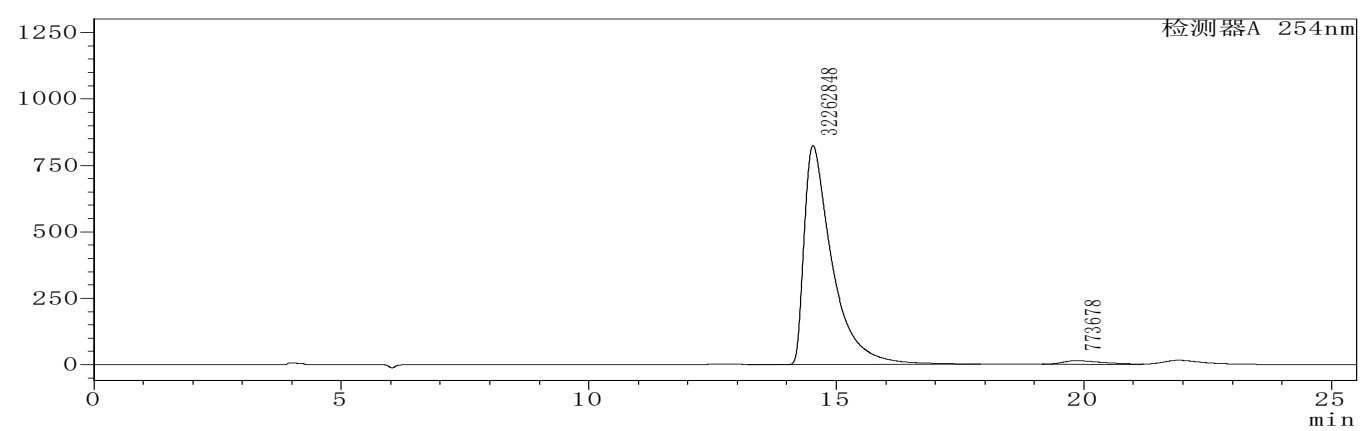

enantio-enriched

\begin{tabular}{|c|c|c|c|}
\hline & Retention Time & Area & \%Area \\
\hline 1 & 14.527 & 32262848 & 97.658 \\
\hline 2 & 19.857 & 773678 & 2.342 \\
\hline
\end{tabular}

tert-Butyl (S)-(1-(2-methyl-4-oxo-4H-chromen-3-yl)propyl)carbamate (9c)

yield $(29.2 \mathrm{mg}$ ), colorless oil, $95 \%$ ee. HPLC (chiral ID column),
$\begin{aligned} & \text { hexane } / i-P r O H \\ & \text { (minor) }=8.34 \mathrm{~min}, t \mathrm{r} \text { (major) }=12.17 \mathrm{~min} .\end{aligned}$ $[\alpha]^{25}{ }_{\mathrm{D}}=-97.2\left(\mathrm{c}=1.0\right.$ in $\left.\mathrm{CH}_{2} \mathrm{Cl}_{2}\right)$.

${ }^{1} \mathrm{H}$ NMR $\left(400 \mathrm{MHz}, \mathrm{CDCl}_{3}\right) \delta 8.14(\mathrm{dd}, J=8.0,1.2 \mathrm{~Hz}, 1 \mathrm{H}), 7.68-7.60(\mathrm{~m}, 1 \mathrm{H})$, $7.38(\mathrm{dd}, J=16.0,8.0 \mathrm{~Hz}, 2 \mathrm{H}), 6.25(\mathrm{~d}, J=9.8 \mathrm{~Hz}, 1 \mathrm{H}), 4.62(\mathrm{dd}, J=17.4,8.0 \mathrm{~Hz}$, $1 \mathrm{H}), 2.56(\mathrm{~s}, 3 \mathrm{H}), 2.04-1.81(\mathrm{~m}, 2 \mathrm{H}), 1.40(\mathrm{~s}, 9 \mathrm{H}), 0.90(\mathrm{t}, J=7.4 \mathrm{~Hz}, 3 \mathrm{H}) .{ }^{13} \mathrm{C}\{1 \mathrm{H}\}$ NMR $\left(150 \mathrm{MHz}, \mathrm{CDCl}_{3}\right) \delta 178.2,163.6,156.0,133.6,125.6,124.9,123.7,120.6$, 117.8, 79.1, 51.4, 28.6, 27.4, 18.7, 11.3. HRMS (FTMS-ESI), m/z calcd for $\mathrm{C}_{18} \mathrm{H}_{23} \mathrm{NNaO}_{4}^{+}\left([\mathrm{M}+\mathrm{Na}]^{+}\right) 340.1519$, found 340.1522 . 


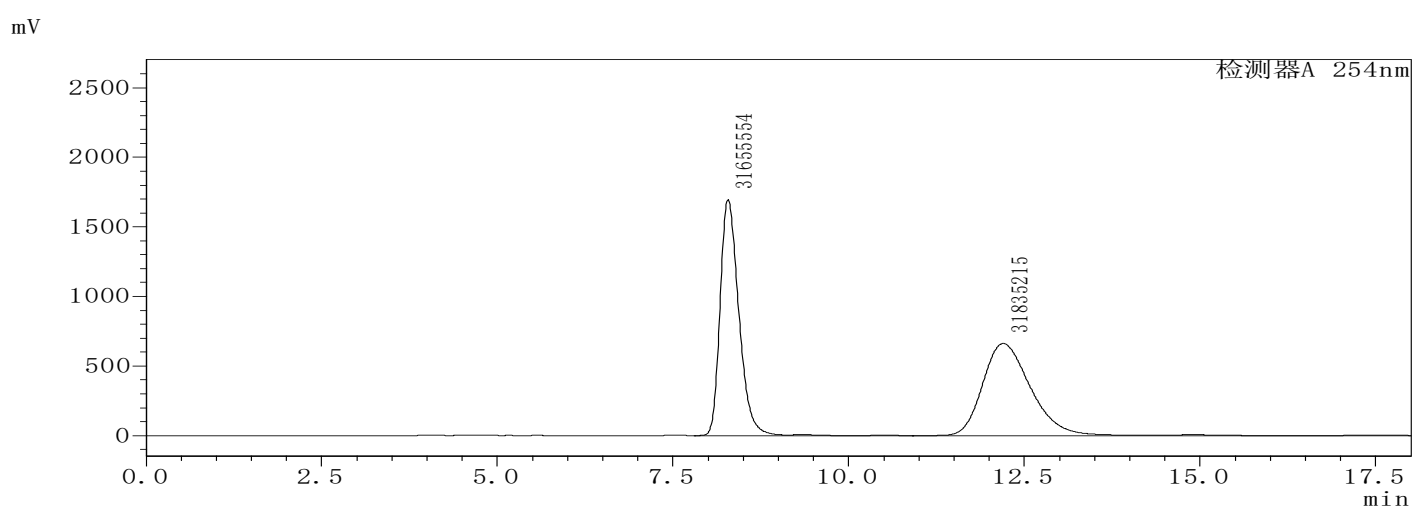

racemic

\begin{tabular}{|c|c|c|c|}
\hline & Retention Time & Area & \%Area \\
\hline 1 & 8.283 & 31655554 & 49.859 \\
\hline 2 & 12.200 & 31835215 & 50.141 \\
\hline
\end{tabular}

$\mathrm{mV}$

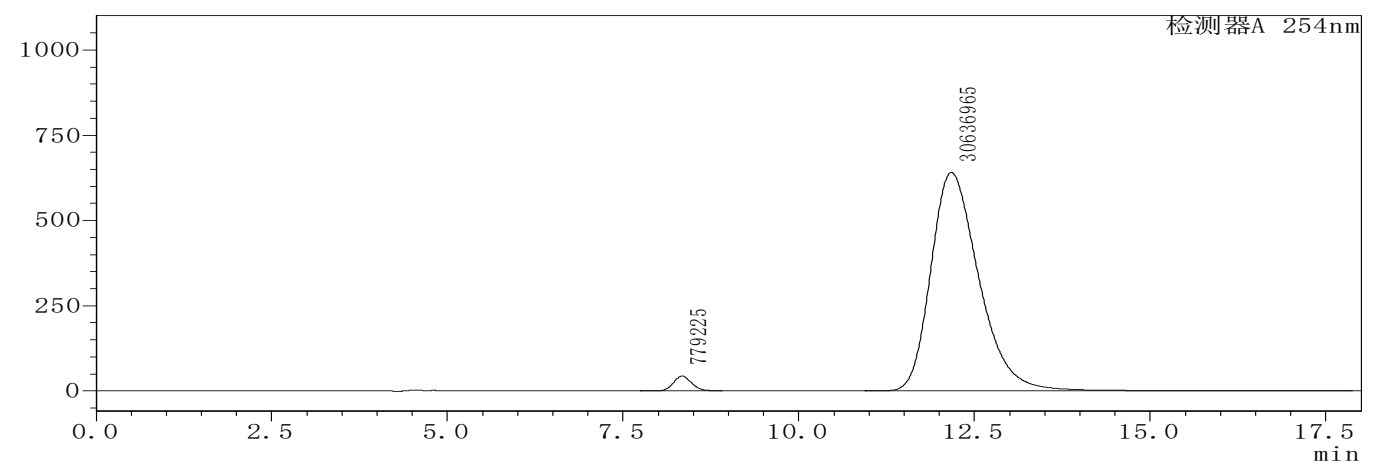

enantio-enriched

\begin{tabular}{|c|c|c|c|}
\hline & Retention Time & Area & \%Area \\
\hline 1 & 8.338 & 779225 & 2.480 \\
\hline 2 & 12.170 & 30636965 & 97.520 \\
\hline
\end{tabular}

(R)-2-(((Benzyloxy)carbonyl)amino)-2-(2-methyl-4-oxo-4H-chromen-3-yl)acetic acid $(6 \mathbf{j})$

$\mathrm{O} \quad \mathrm{NHCO}_{2} \mathrm{Bn}$ Compound 6j: Prepared in $0.26 \mathrm{mmol}$ scale. $84 \%$ yield $(80.0$<smiles>C=C1C(C(C)C(=O)O)=C(C)Oc2ccccc21</smiles>
$\mathrm{mg})$, colorless oil.

$[\alpha]^{25} \mathrm{D}=-55.7\left(\mathrm{c}=1.0\right.$ in $\left.\mathrm{CH}_{2} \mathrm{Cl}_{2}\right)$.

${ }^{1} \mathrm{H}$ NMR $\left(400 \mathrm{MHz}, \mathrm{CDCl}_{3}\right) \delta 8.08(\mathrm{~s}, 1 \mathrm{H}), 7.62(\mathrm{t}, J=7.4$

$\mathrm{Hz}, 1 \mathrm{H}), 7.38(\mathrm{~d}, J=8.0 \mathrm{~Hz}, 1 \mathrm{H}), 7.35-7.21(\mathrm{~m}, 7 \mathrm{H}), 6.59(\mathrm{~s}, 1 \mathrm{H}), 5.41(\mathrm{~s}, 1 \mathrm{H}), 5.02$ $(\mathrm{q}, J=12.4 \mathrm{~Hz}, 2 \mathrm{H}), 2.66(\mathrm{~s}, 3 \mathrm{H}) .{ }^{13} \mathrm{C}\{1 \mathrm{H}\} \mathrm{NMR}\left(100 \mathrm{MHz}, \mathrm{CDCl}_{3}\right) \delta 166.2,156.4$, 156.2, 136.2, 134.1, 128.5, 128.1, 127.9, 125.9, 125.3, 122.9, 119.4, 117.9, 67.1, 51.9, 18.7. HRMS (FTMS-ESI), $\mathrm{m} / \mathrm{z}$ calcd for $\mathrm{C}_{20} \mathrm{H}_{17} \mathrm{NNaO}_{6}{ }^{+}\left([\mathrm{M}+\mathrm{Na}]^{+}\right) 390.0948$, found 390.0947.

tert-Butyl ((S)-1-(2-methyl-4-oxo-4H-chromen-3-yl)-3-((8R,9S,13S,14S)-13-methyl-6,7,8,9,11,12,13,14,15,16-decahydrospiro[cyclopenta[a]phenanthrene-17,2' -[1,3 ]dioxolan]-3-yl)prop-2-yn-1-yl)carbamate (3z) 


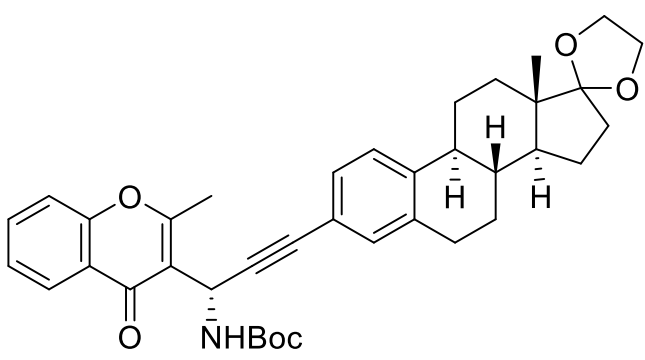

Compound 3z: Prepared in $0.05 \mathrm{mmol}$ scale using 1.5 equiv of $1 \mathrm{a}$ at $80{ }^{\circ} \mathrm{C}$ for 62 h. $86 \%$ yield $(26.2 \mathrm{mg})$, yellow oil, >19:1 dr. $[\alpha]^{25} \mathrm{D}=+45.4\left(\mathrm{c}=1.0\right.$ in $\left.\mathrm{CH}_{2} \mathrm{Cl}_{2}\right)$.

${ }^{1} \mathrm{H}$ NMR $\left(500 \mathrm{MHz}, \mathrm{CDCl}_{3}\right) \delta 8.20(\mathrm{~d}, J=$ $8.0 \mathrm{~Hz}, 1 \mathrm{H}), 7.64(\mathrm{t}, J=7.6 \mathrm{~Hz}, 1 \mathrm{H}), 7.46-$ $7.36(\mathrm{~m}, 2 \mathrm{H}), 7.15(\mathrm{~d}, J=15.4 \mathrm{~Hz}, 3 \mathrm{H}), 6.52$ $(\mathrm{d}, J=8.4 \mathrm{~Hz}, 1 \mathrm{H}), 5.86(\mathrm{~d}, J=9.2 \mathrm{~Hz}, 1 \mathrm{H}), 3.99-3.81(\mathrm{~m}, 4 \mathrm{H}), 2.84-2.73(\mathrm{~m}, 2 \mathrm{H})$, $2.64(\mathrm{~s}, 3 \mathrm{H}), 2.29(\mathrm{~d}, J=13.2 \mathrm{~Hz}, 1 \mathrm{H}), 2.23(\mathrm{t}, J=10.6 \mathrm{~Hz}, 1 \mathrm{H}), 2.09-1.97(\mathrm{~m}, 1 \mathrm{H})$, $1.90-1.81(\mathrm{~m}, 2 \mathrm{H}), 1.78-1.69(\mathrm{~m}, 3 \mathrm{H}), 1.61(\mathrm{dd}, J=18.4,10.8 \mathrm{~Hz}, 1 \mathrm{H}), 1.52(\mathrm{~d}, J$ $=12.6 \mathrm{~Hz}, 1 \mathrm{H}), 1.43(\mathrm{~s}, 9 \mathrm{H}), 1.37-1.31(\mathrm{~m}, 3 \mathrm{H}), 0.86(\mathrm{~s}, 3 \mathrm{H}) .{ }^{13} \mathrm{C}\{1 \mathrm{H}\}$ NMR $(150$ $\left.\mathrm{MHz}, \mathrm{CDCl}_{3}\right) \delta 177.1,163.4,156.0,155.2,141.0,136.8,133.7,132.5,129.1,125.9$, 125.3, 125.1, 123.7, 119.8, 119.5, 117.9, 85.9, 82.3, 80.0, 65.4, 64.7, 49.7, 46.2, 44.2, 41.1, 38.8, 34.3, 30.8, 29.3, 28.5, 26.9, 25.9, 22.5, 18.6, 14.4. HRMS (FTMS-ESI), $\mathrm{m} / \mathrm{z}$ calcd for $\mathrm{C}_{38} \mathrm{H}_{44} \mathrm{NO}_{6}{ }^{+}\left([\mathrm{M}+\mathrm{H}]^{+}\right) 610.3163$, found 610.3159 .

tert-Butyl (1-ethoxy-3-((8R,9S,13S,14S)-13-methyl-6,7,8,9,11,12,13,14,15,16-decahydrospiro[cyclopenta[a]phenanthrene-17,2'-[1,3]dioxolan]-3-yl)prop-2-yn-1-yl)c arbamate (2z)

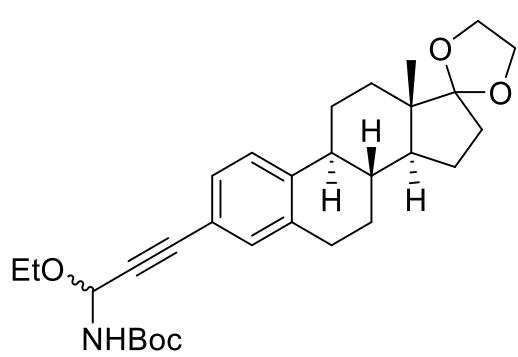

${ }^{1} \mathrm{H}$ NMR $\left(400 \mathrm{MHz}, \mathrm{CDCl}_{3}\right) \delta 7.25-7.15(\mathrm{~m}, 3 \mathrm{H})$, $5.82(\mathrm{~d}, J=9.2 \mathrm{~Hz}, 1 \mathrm{H}), 5.46(\mathrm{~d}, J=8.4 \mathrm{~Hz}, 1 \mathrm{H})$, $3.99-3.85(\mathrm{~m}, 4 \mathrm{H}), 3.72-3.61(\mathrm{~m}, 2 \mathrm{H}), 2.87-2.73$ (m, 2H), $2.39-2.22(\mathrm{~m}, 2 \mathrm{H}), 2.08-1.97(\mathrm{~m}, 1 \mathrm{H})$, $1.93-1.84(\mathrm{~m}, 2 \mathrm{H}), 1.83-1.72(\mathrm{~m}, 2 \mathrm{H}), 1.68-1.58$ $(\mathrm{m}, 2 \mathrm{H}), 1.56-1.43(\mathrm{~m}, 9 \mathrm{H}), 1.44-1.41(\mathrm{~m}, 1 \mathrm{H})$, $1.40-1.34(\mathrm{~m}, 2 \mathrm{H}), 1.33-1.30(\mathrm{~m}, 1 \mathrm{H}), 1.26(\mathrm{t}, J=$ $7.0 \mathrm{~Hz}, 3 \mathrm{H}), 0.88(\mathrm{~s}, 3 \mathrm{H}) .{ }^{13} \mathrm{C}\{1 \mathrm{H}\} \mathrm{NMR}\left(100 \mathrm{MHz}, \mathrm{CDCl}_{3}\right) \delta 141.8,137.0,132.4$, 129.1, 125.5, 119.5, 118.8, 84.9, 84.2, 80.7, 72.4, 65.4, 64.7, 62.8, 49.6, 46.2, 44.3, 38.7, 34.3, 30.8, 29.3, 28.4, 26.9, 25.9, 22.5, 15.2, 14.5. HRMS (FTMS-ESI), m/z calcd for $\mathrm{C}_{30} \mathrm{H}_{41} \mathrm{NNaO}_{5}^{+}\left([\mathrm{M}+\mathrm{Na}]^{+}\right)$518.2877, found 518.2881.

\section{Benzyl N-((benzyloxy)carbonyl)-N-((tert-butoxycarbonyl)oxy)glycinate (5a)}

BocO ${ }_{\mathrm{N}^{-}} \mathrm{Cbz} \quad{ }^{1} \mathrm{H}$ NMR $\left(400 \mathrm{MHz}, \mathrm{CDCl}_{3}\right) \delta 7.34(\mathrm{~d}, J=8.8 \mathrm{~Hz}, 10 \mathrm{H}), 5.22$ (s, $2 \mathrm{H}), 5.19(\mathrm{~s}, 2 \mathrm{H}), 4.44(\mathrm{~s}, 2 \mathrm{H}), 1.49(\mathrm{~s}, 9 \mathrm{H}),{ }^{13} \mathrm{C}\{1 \mathrm{H}\}$ NMR $(100$

$\left.\mathrm{CO}_{2} \mathrm{Bn} \quad \mathrm{MHz}, \mathrm{CDCl}_{3}\right) \delta 167.3,156.0,151.8,135.2,135.1,128.6,128.5$, 128.4, 128.0, 85.6, 68.8, 67.3, 52.3, 27.6. HRMS (FTMS-ESI), m/z calcd for $\mathrm{C}_{22} \mathrm{H}_{25} \mathrm{NNaO}_{7}^{+}\left([\mathrm{M}+\mathrm{Na}]^{+}\right) 438.1523$, found 438.1513 . 


\section{Copies of NMR Specta}

3a- ${ }^{1} \mathrm{H}$ NMR (400 MHz, $\left.\mathrm{CDCl}_{3}\right)$

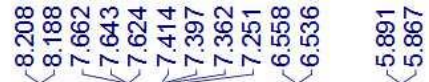

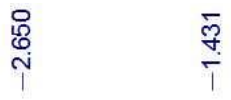<smiles>Cc1oc2ccccc2c(=O)c1[C@H](C#Cc1ccccc1)NC(C)(C)C</smiles>

3a

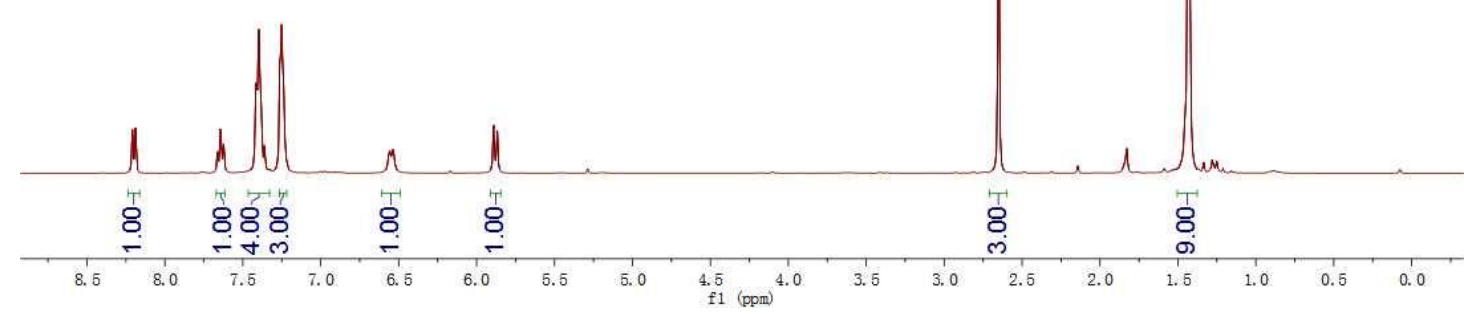

3a- ${ }^{13} \mathrm{C}\{1 \mathrm{H}\}$ NMR (100 MHz, $\left.\mathrm{CDCl}_{3}\right)$

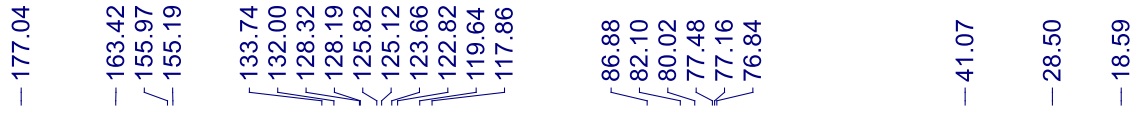<smiles>Cc1oc2ccccc2c(=O)c1[C@H](C#Cc1ccccc1)NC(=O)OC(C)(C)C</smiles>

3a

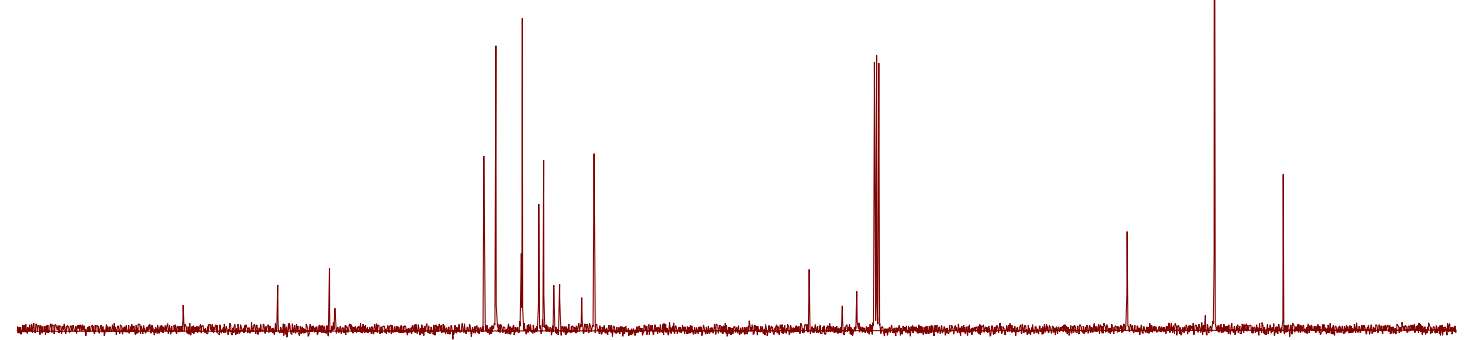

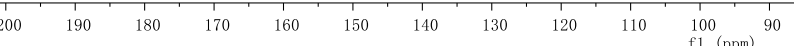


3b- ${ }^{1} \mathrm{H}$ NMR (400 $\left.\mathrm{MHz}, \mathrm{CDCl}_{3}\right)$

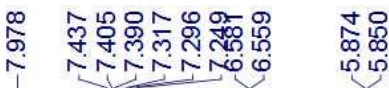

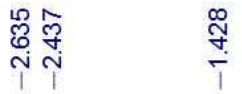

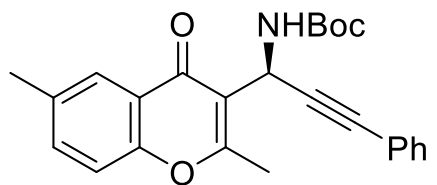

$3 b$

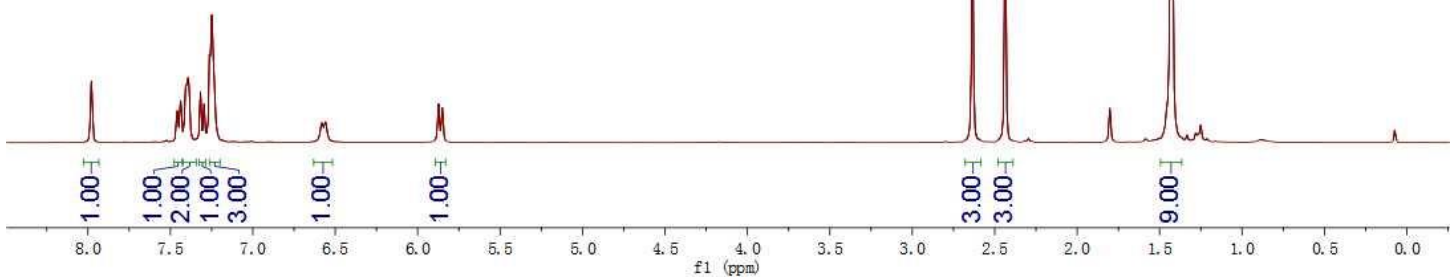

3b- ${ }^{13} \mathrm{C}\{1 \mathrm{H}\}$ NMR (100 MHz, $\left.\mathrm{CDCl}_{3}\right)$

\begin{tabular}{|c|c|c|c|}
\hline 赵 & 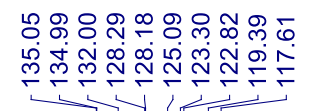 & 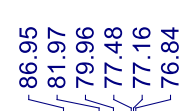 & 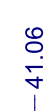 \\
\hline
\end{tabular}<smiles>Cc1ccc2oc(C)c([C@H](C#Cc3ccccc3)N[R16](C)(C)C)c(=O)c2c1</smiles>

3b
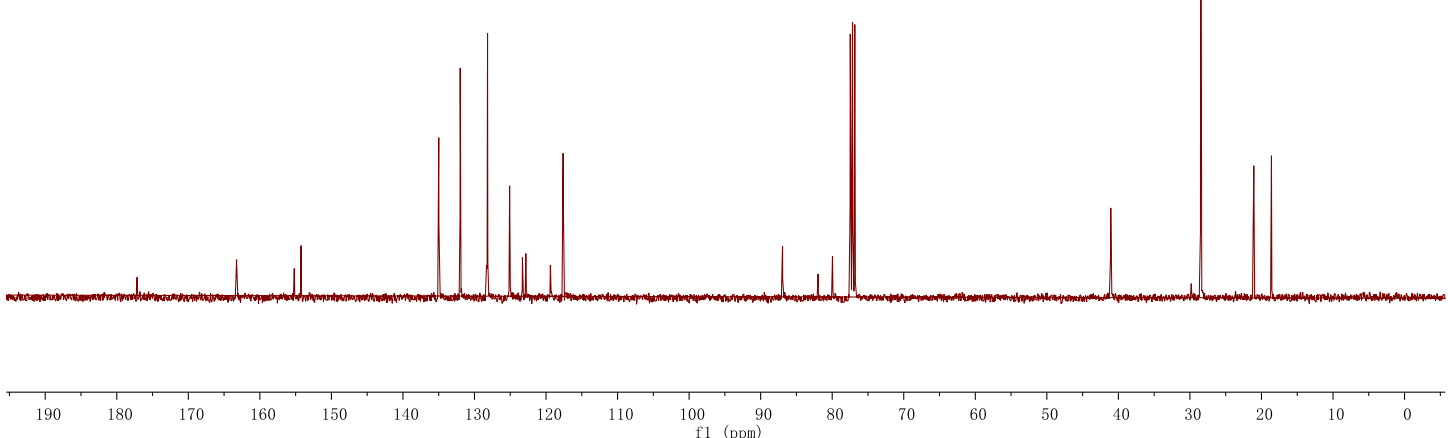
3c- ${ }^{1} \mathrm{H}$ NMR (400 MHz, CDCl3)

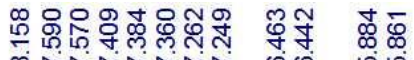
कriñNiñ<smiles>Cc1oc2ccc(Cl)cc2c(=O)c1[C@H](C#Cc1ccccc1)NC(C)(C)C</smiles>

$3 c$

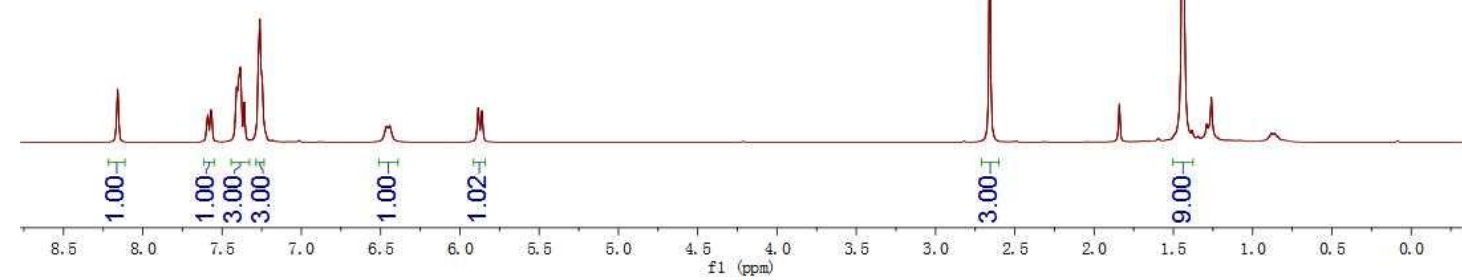

3c- ${ }^{13} \mathrm{C}\{1 \mathrm{H}\}$ NMR (125 MHz, $\left.\mathrm{CDCl}_{3}\right)$

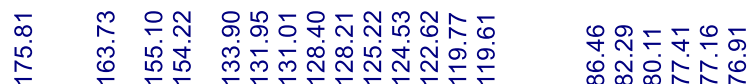<smiles>Cc1oc2ccc(Cl)cc2c(=O)c1[C@H](C#Cc1ccccc1)NC(C)(C)C</smiles>

$3 c$

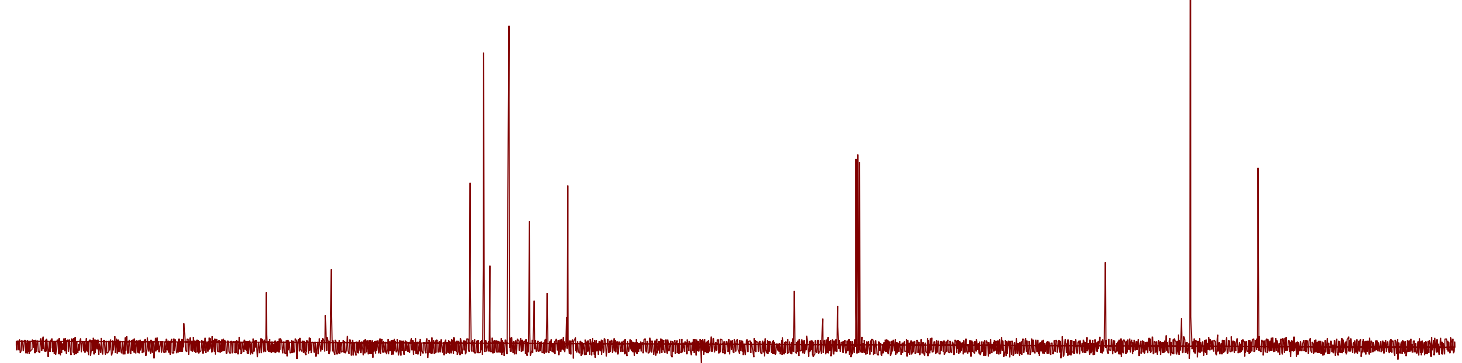

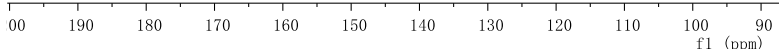


3d- ${ }^{1} \mathrm{H}$ NMR (400 MHz, CDCl3)

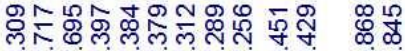

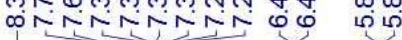<smiles>Cc1oc2ccc(Br)cc2c(=O)c1[C@H](C#Cc1ccccc1)NC(=O)OC(C)(C)C</smiles>

3d

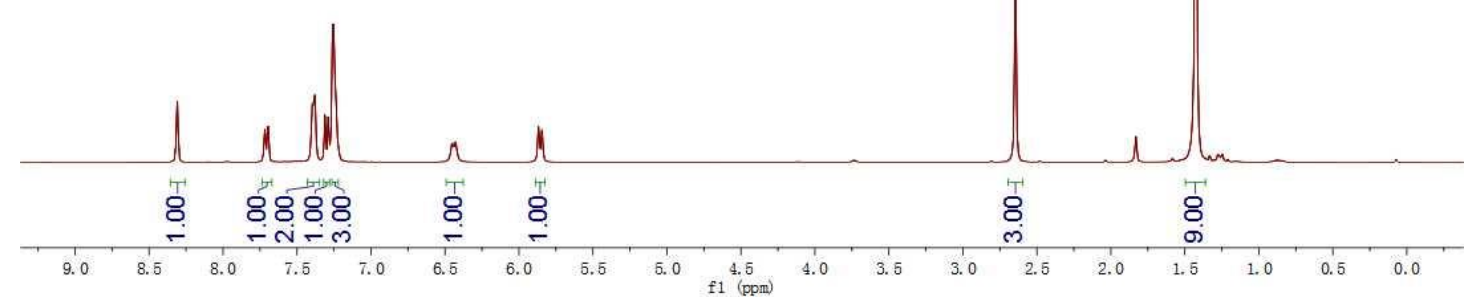

3d- ${ }^{13} \mathrm{C}\{1 \mathrm{H}\}$ NMR (100 MHz, $\left.\mathrm{CDCl}_{3}\right)$

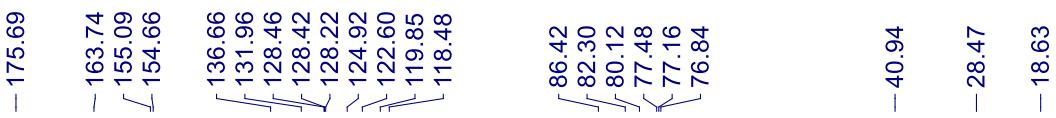<smiles>Cc1oc2ccc(Br)cc2c(=O)c1[C@H](C#Cc1ccccc1)C(=O)OC(C)(C)C</smiles>

3d

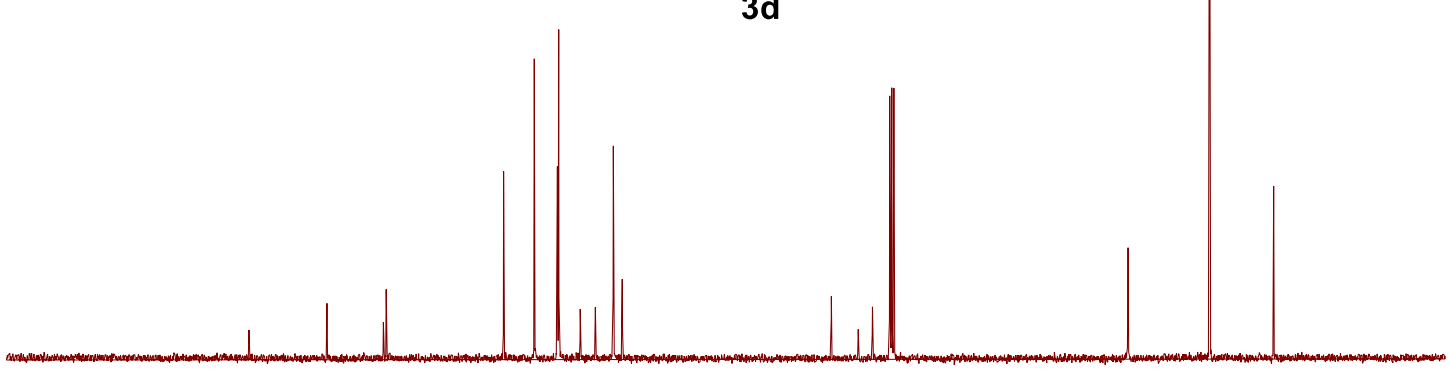

$210 \quad 200 \quad 190 \quad 180$ 
3e- ${ }^{1} \mathrm{H}$ NMR (400 MHz, CDCl3)

ก๊

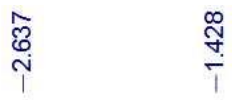<smiles>Cc1oc2cc(Br)ccc2c(=O)c1[C@H](C#Cc1ccccc1)NC(=O)OC(C)(C)C</smiles>

$3 e$

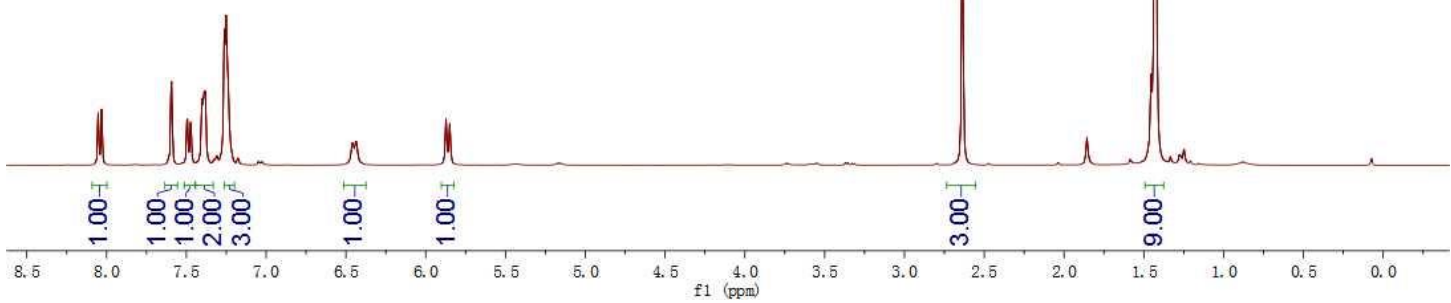

3e- ${ }^{13} \mathrm{C}\{1 \mathrm{H}\}$ NMR (100 MHz, $\left.\mathrm{CDCl}_{3}\right)$

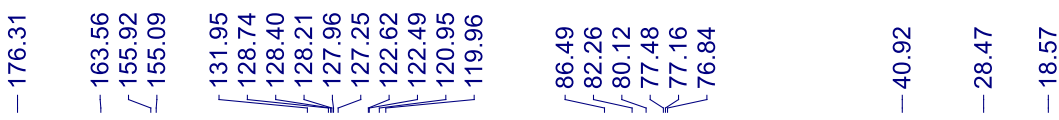
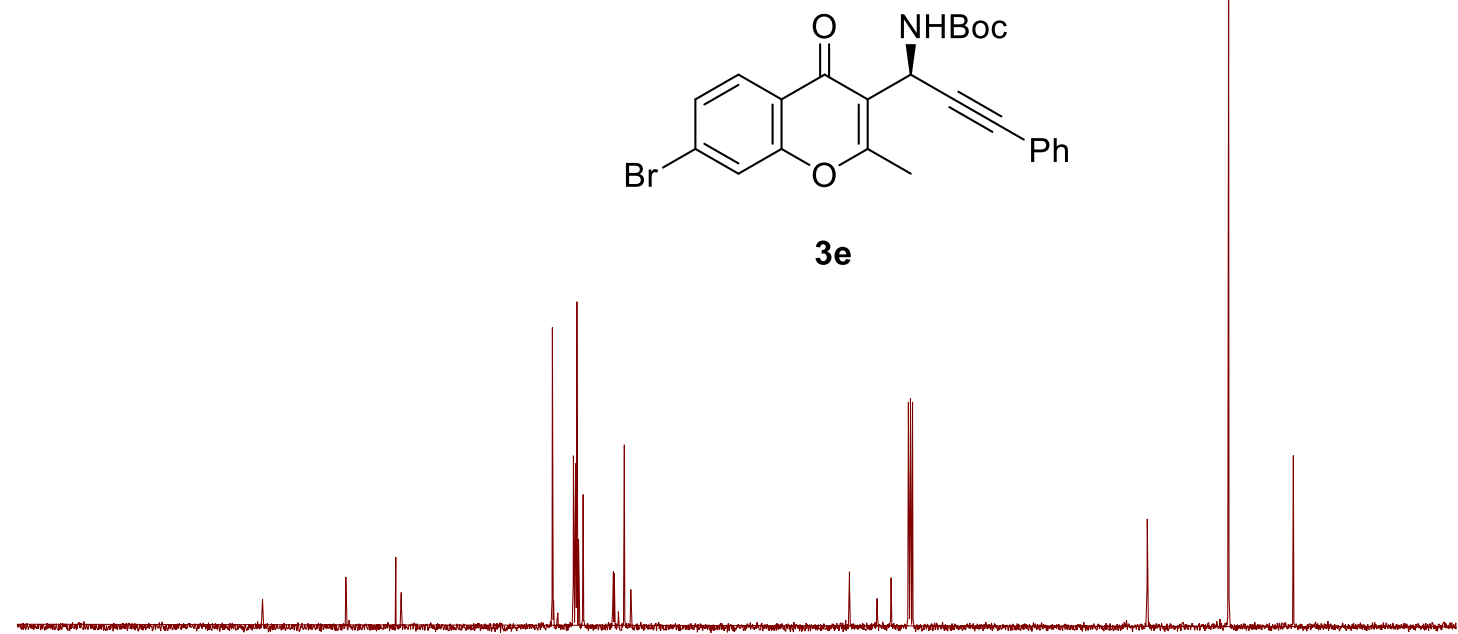

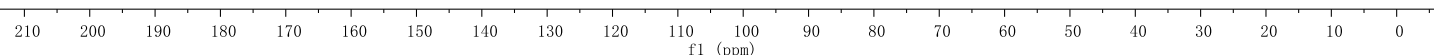


3f- ${ }^{1} \mathrm{H}$ NMR (400 MHz, CDCl3)

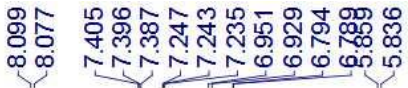

$\bar{\infty}$
$\infty$
$i$
$i$

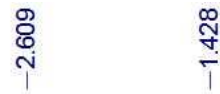<smiles>COc1ccc2c(=O)c([C@H](C#Cc3ccccc3)NC(=O)OC(C)(C)C)c(C)oc2c1</smiles>

$3 f$

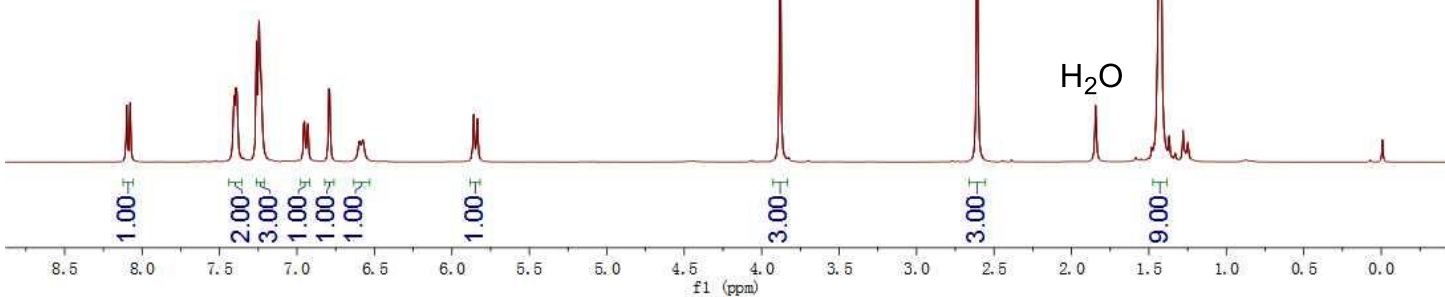

3f- ${ }^{13} \mathrm{C}\{1 \mathrm{H}\}$ NMR (100 $\left.\mathrm{MHz}, \mathrm{CDCl}_{3}\right)$

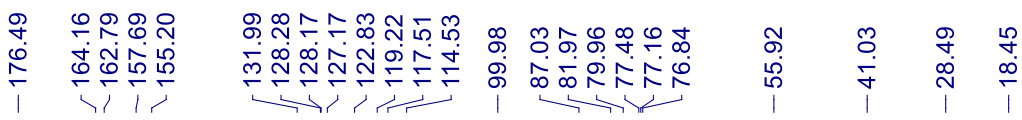<smiles>COc1ccc2c(=O)c([C@H](C#Cc3ccccc3)NC(=O)OCc3ccccc3)c(C)oc2c1</smiles>

$3 f$

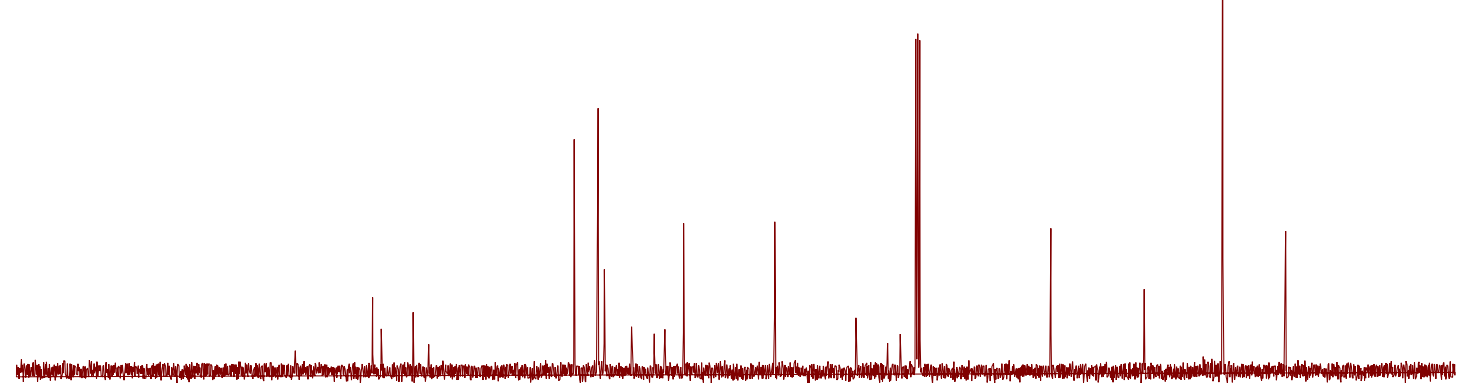

$\begin{array}{lllllllllllll}220 & 210 & 200 & 190 & 180 & 170 & 160 & 150 & 140 & 130 & 120 & 110 & 100 \\ \mathrm{fl}(\mathrm{ppm})\end{array}$ 
3g- ${ }^{1} \mathrm{H}$ NMR (400 MHz, CDCl3)

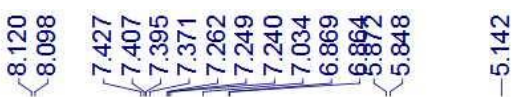

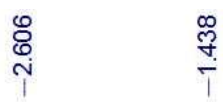

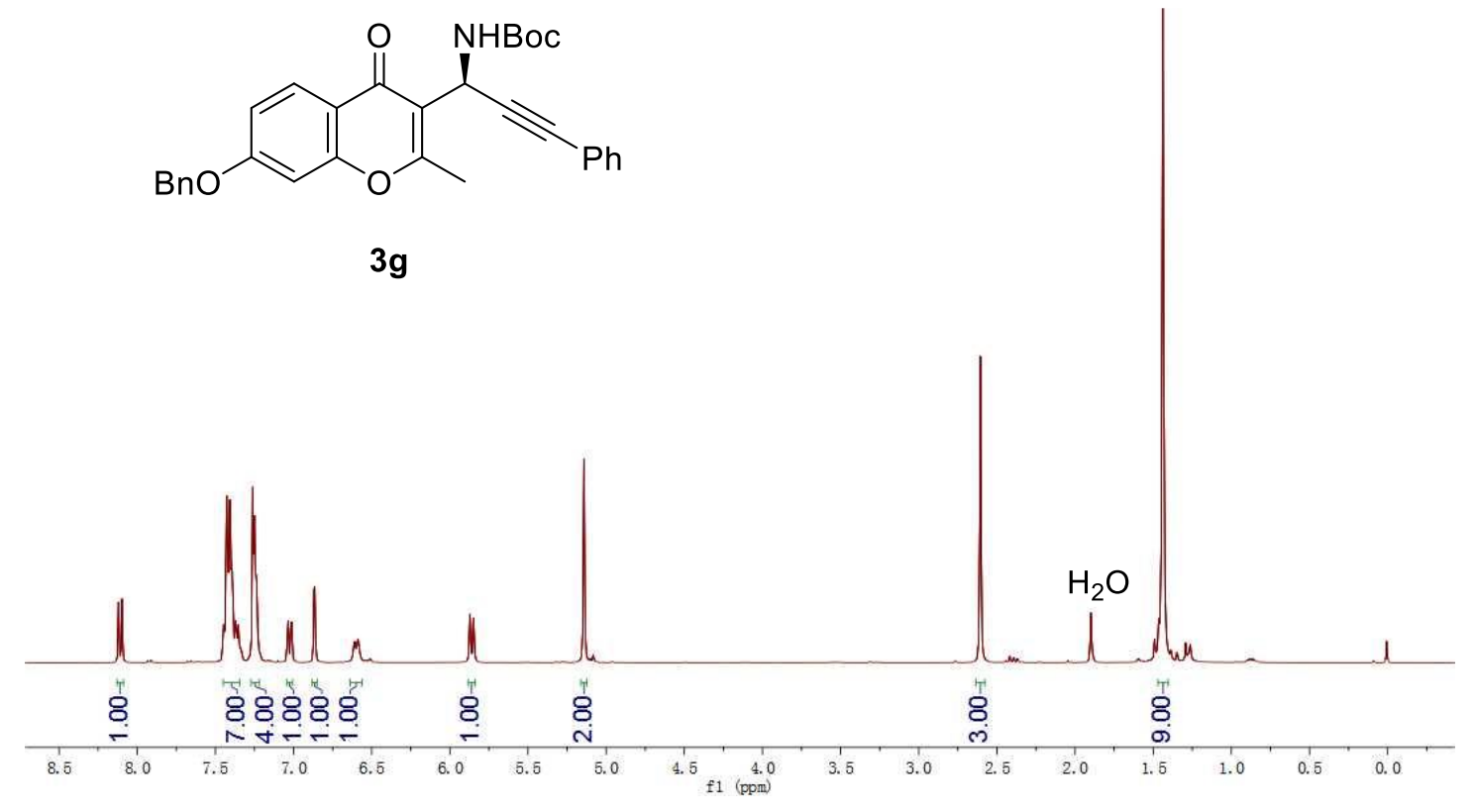

$3 g$

\section{in}

$\frac{1}{+}$

3g- ${ }^{13} \mathrm{C}\{1 \mathrm{H}\}$ NMR (100 MHz, $\left.\mathrm{CDCl}_{3}\right)$

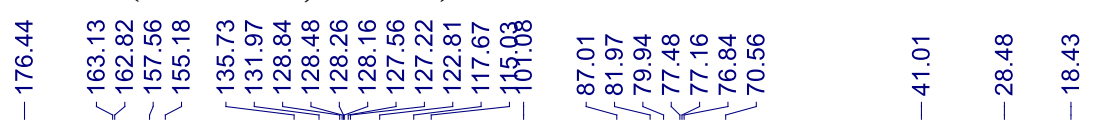<smiles>Cc1oc2cc(OCc3ccccc3)ccc2c(=O)c1[C@H](C#Cc1ccccc1)C(=O)OCc1ccccc1</smiles>

$3 g$

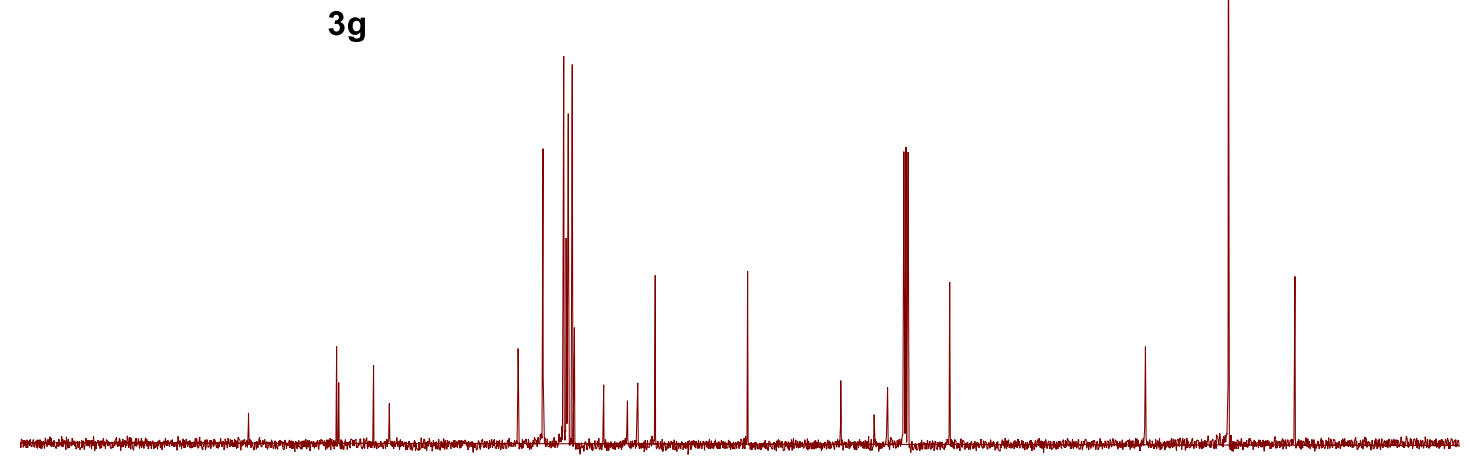

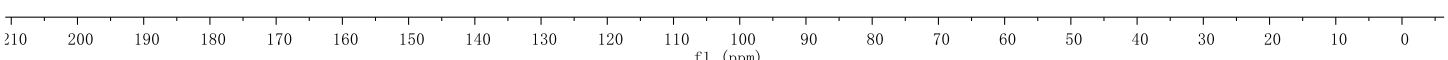


3h- ${ }^{1} \mathrm{H}$ NMR (400 MHz, CDCl3)

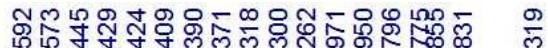

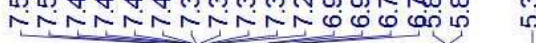

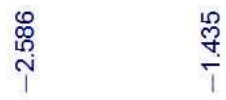

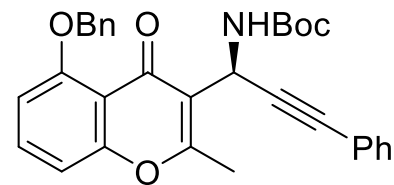

$3 \mathrm{~h}$

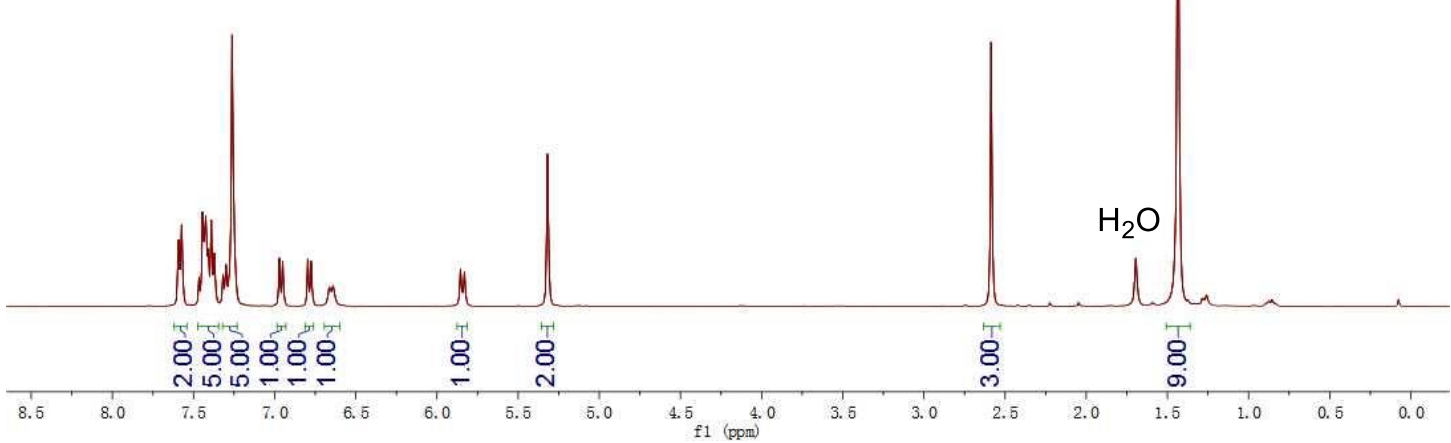

3h- ${ }^{13} \mathrm{C}\{1 \mathrm{H}\}$ NMR (150 MHz, $\left.\mathrm{CDCl}_{3}\right)$

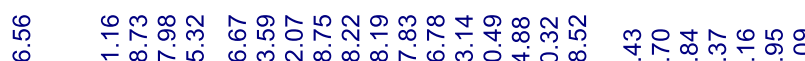

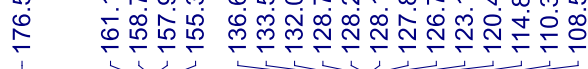

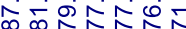

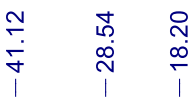<smiles>Cc1oc2cccc(OCc3ccccc3)c2c(=O)c1[C@H](C#Cc1ccccc1)C(=O)OC(C)(C)C</smiles>

$3 h$

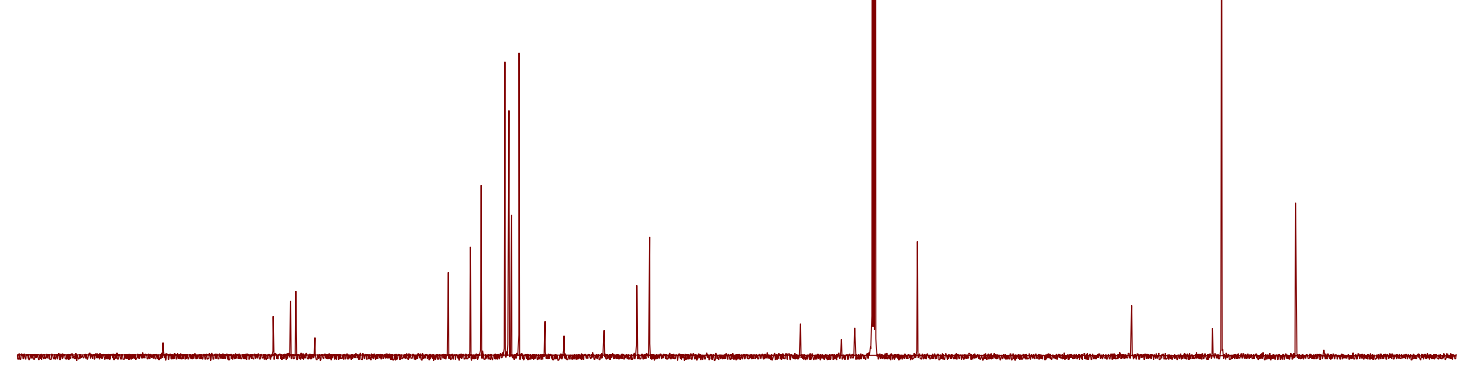

$\begin{array}{llllllllll}190 & 180 & 170 & 160 & 150 & 140 & 130 & 120 & 110 & \begin{array}{l}100 \\ \mathrm{f} 1(\mathrm{ppm})\end{array}\end{array}$ 
3i- ${ }^{1} \mathrm{H}$ NMR (400 MHz, CDCl3)

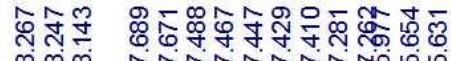<smiles>CC(C)(C)OC(=O)N[C@@H](C#Cc1ccccc1)c1coc2ccccc2c1=O</smiles>

$3 \mathbf{i}$

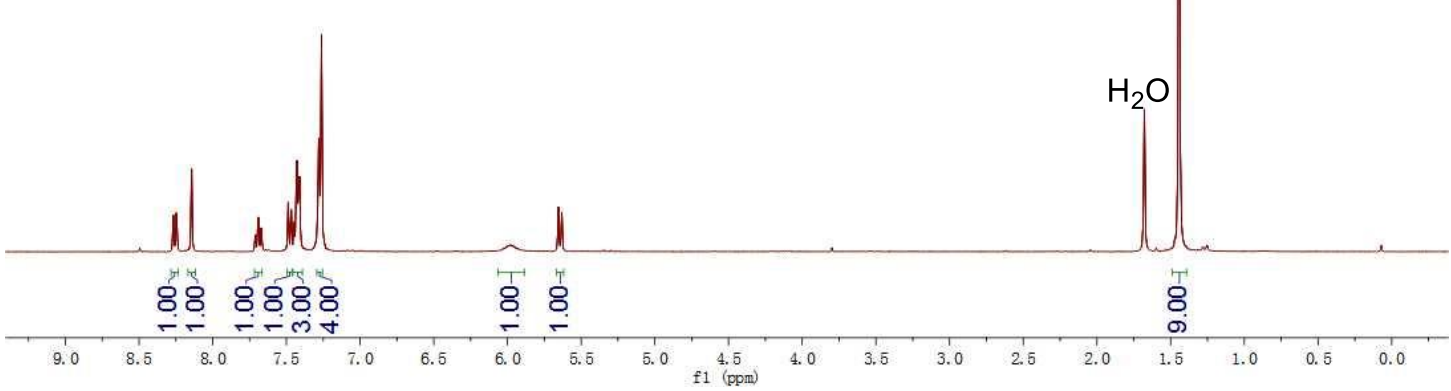

3i- ${ }^{13} \mathrm{C}\{1 \mathrm{H}\}$ NMR (150 MHz, CDCl $)$

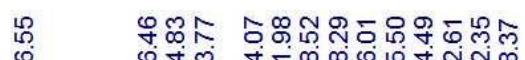

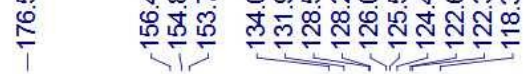<smiles>CC(C)(C)OC(=O)C(C#Cc1ccccc1)c1coc2ccccc2c1=O</smiles>

$3 \mathbf{i}$

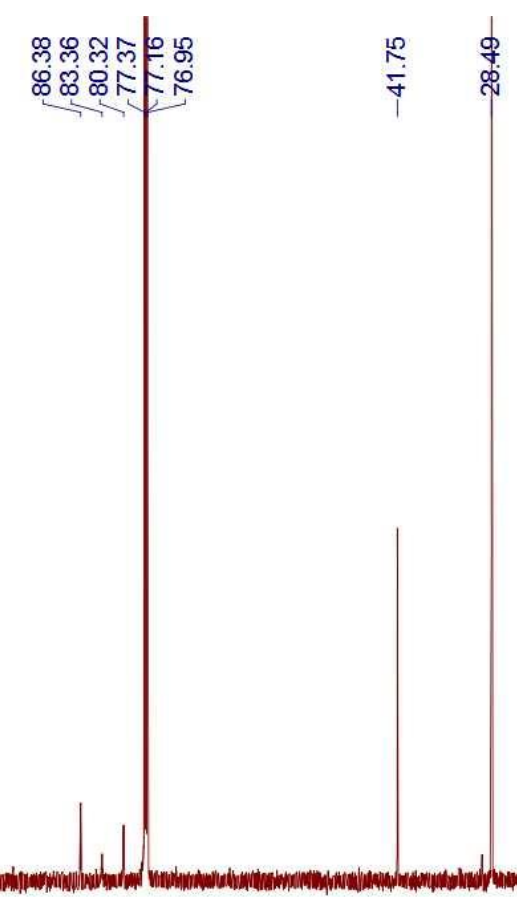

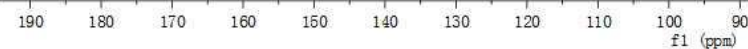


3j- ${ }^{1} \mathrm{H}$ NMR (400 MHz, $\left.\mathrm{CDCl}_{3}\right)$

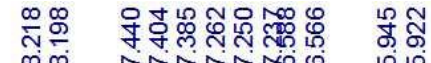

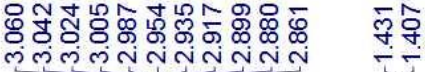<smiles>CCc1oc2ccccc2c(=O)c1[C@H](C#Cc1ccccc1)C(=O)OC(C)(C)C</smiles>

3j

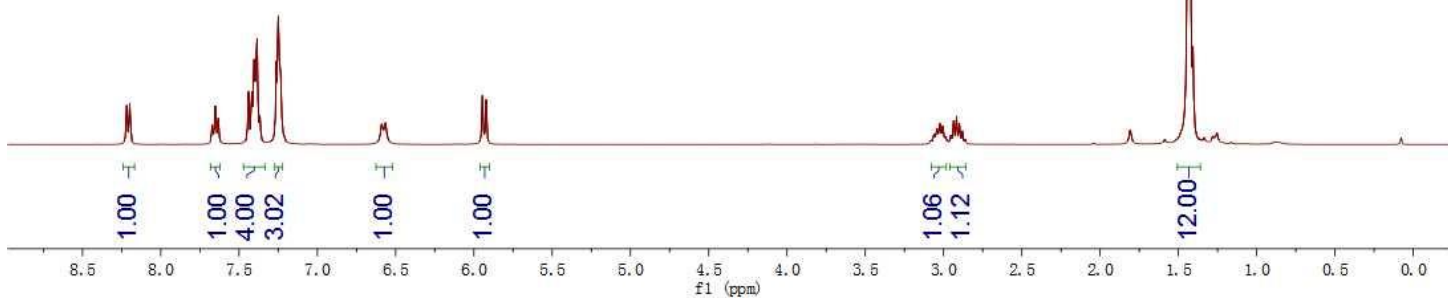

3j- ${ }^{13} \mathrm{C}\{1 \mathrm{H}\}$ NMR (150 MHz, CDCl $)$

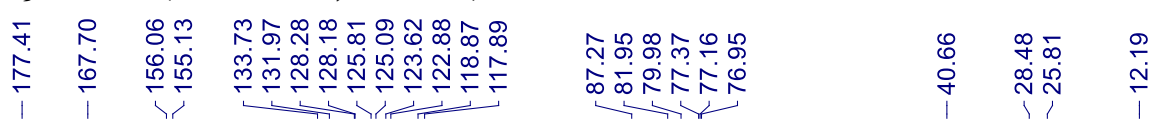<smiles>CCc1oc2ccccc2c(=O)c1[C@H](C#Cc1ccccc1)C(=O)OC(C)(C)C</smiles>

3j

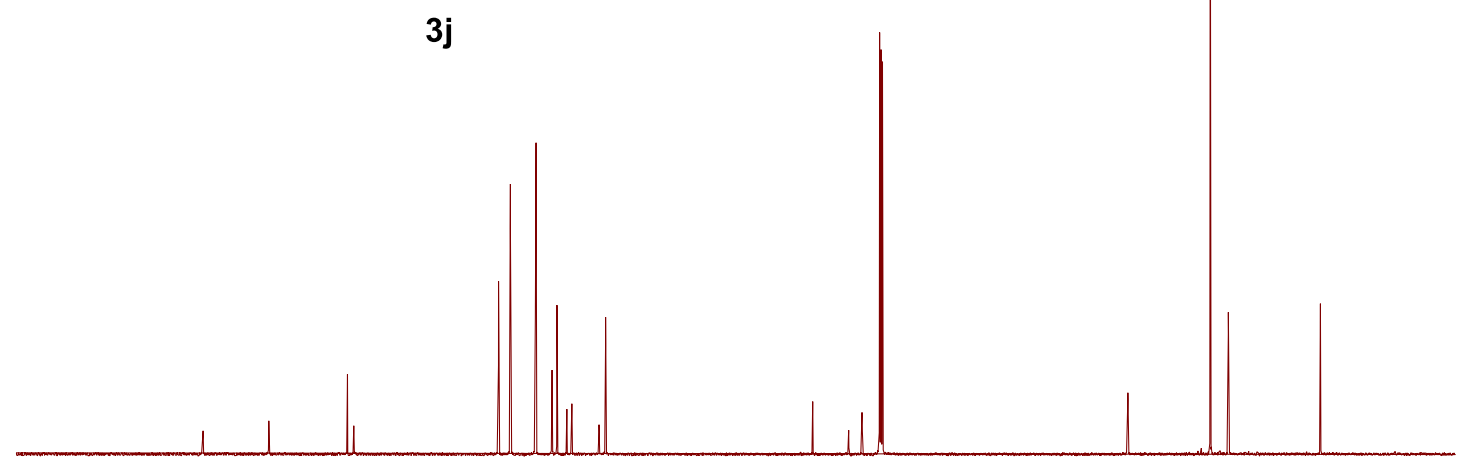

$100 \quad 190 \quad 180$

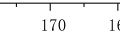

$150 \quad 140$

100 
3k- ${ }^{1} \mathrm{H}$ NMR (400 $\left.\mathrm{MHz}, \mathrm{CDCl}_{3}\right)$

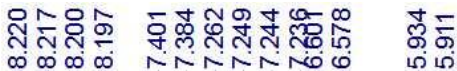<smiles>CCCc1oc2ccccc2c(=O)c1[C@H](C#Cc1ccccc1)C(=O)OC(C)(C)C</smiles>

$3 k$

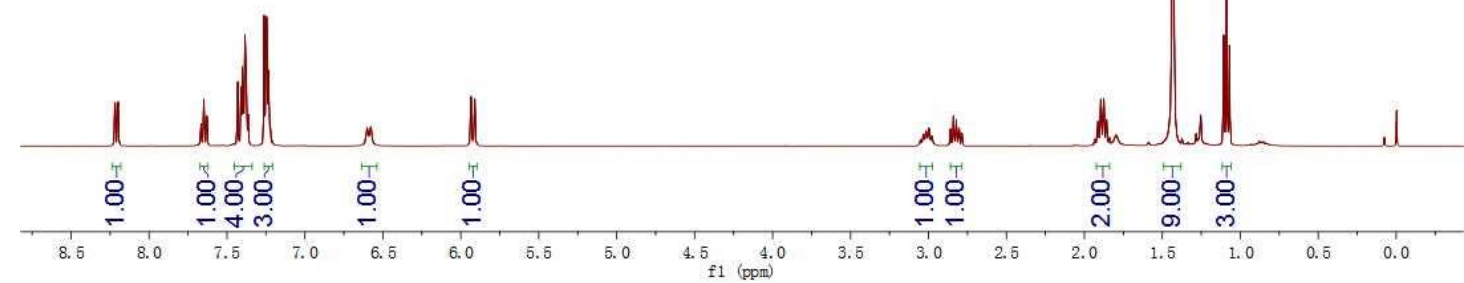

3k- ${ }^{13} \mathrm{C}\{1 \mathrm{H}\}$ NMR (150 $\left.\mathrm{MHz}, \mathrm{CDCl}_{3}\right)$

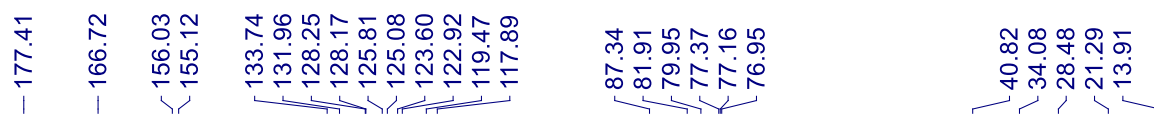

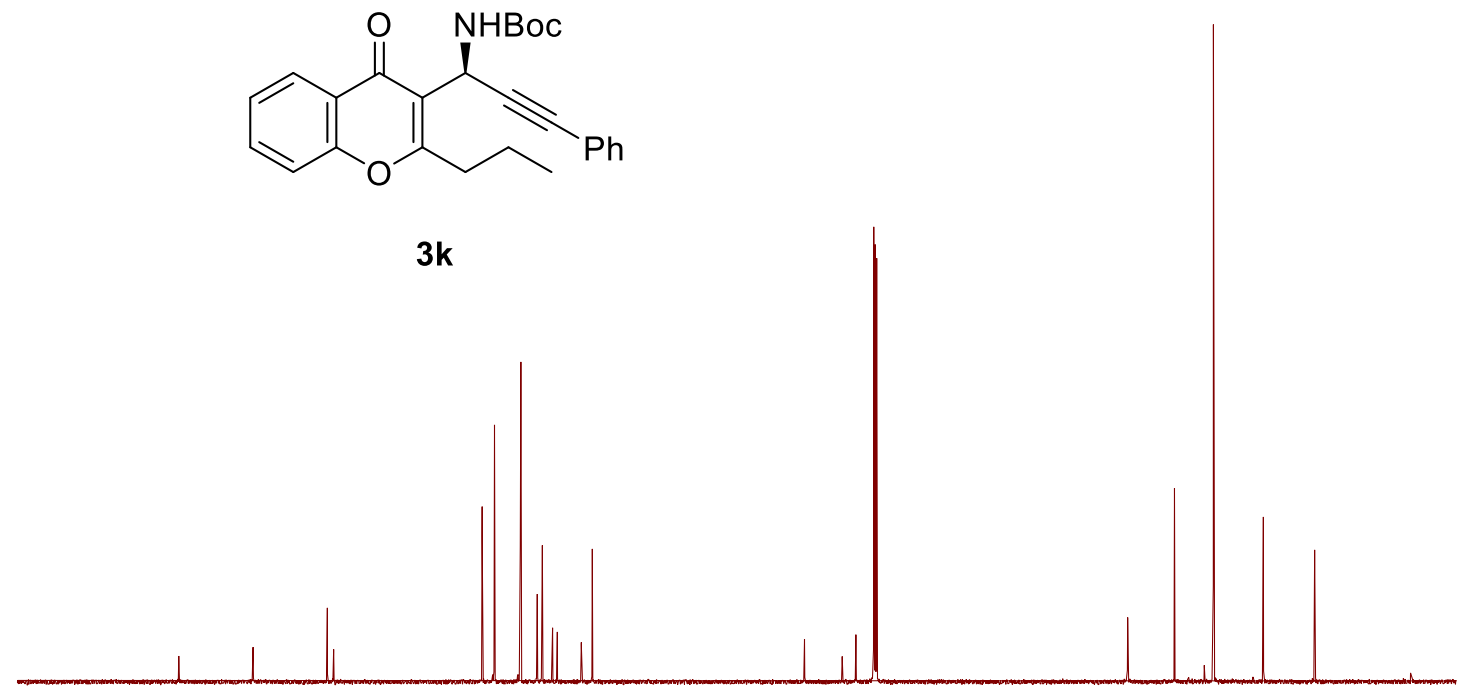

3k
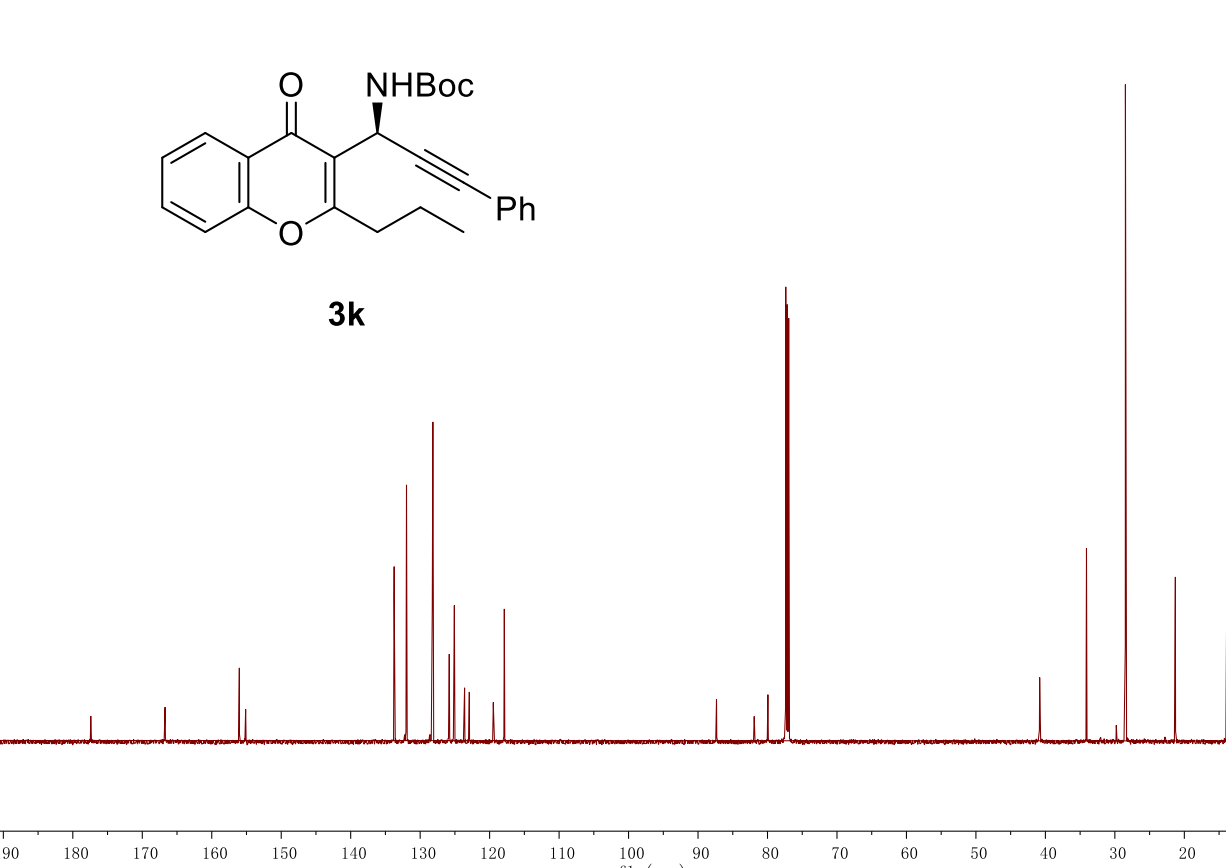

$180 \quad 170$

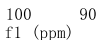




\section{I- ${ }^{1} \mathrm{H}$ NMR (400 MHz, $\left.\mathrm{CDCl}_{3}\right)$}

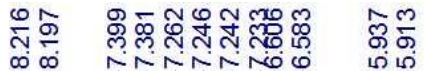<smiles>CCCCCc1oc2ccccc2c(=O)c1[C@H](C#CCc1ccccc1)NC(=O)OC(C)(C)C</smiles>

31

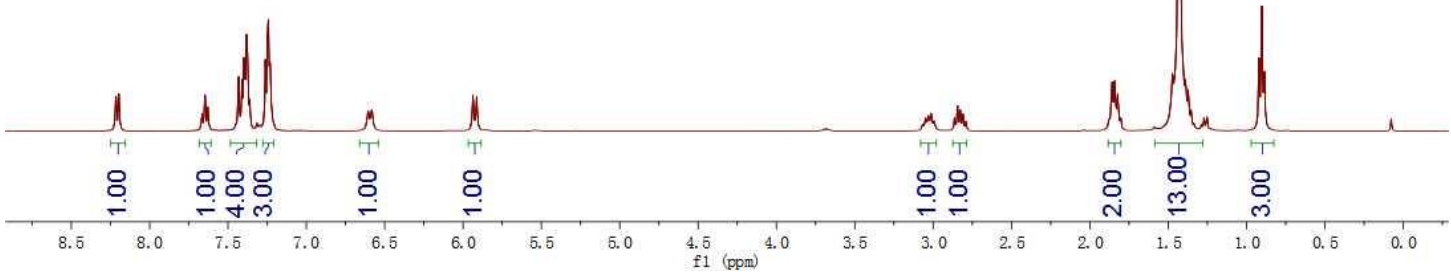

3I- ${ }^{13} \mathrm{C}\{1 \mathrm{H}\}$ NMR (100 MHz, $\left.\mathrm{CDCl}_{3}\right)$

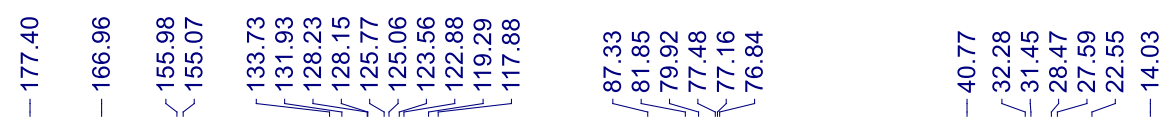<smiles>CCCCCc1oc2ccccc2c(=O)c1[C@H](C#C[N+](=O)[O-])c1ccccc1</smiles>

3!

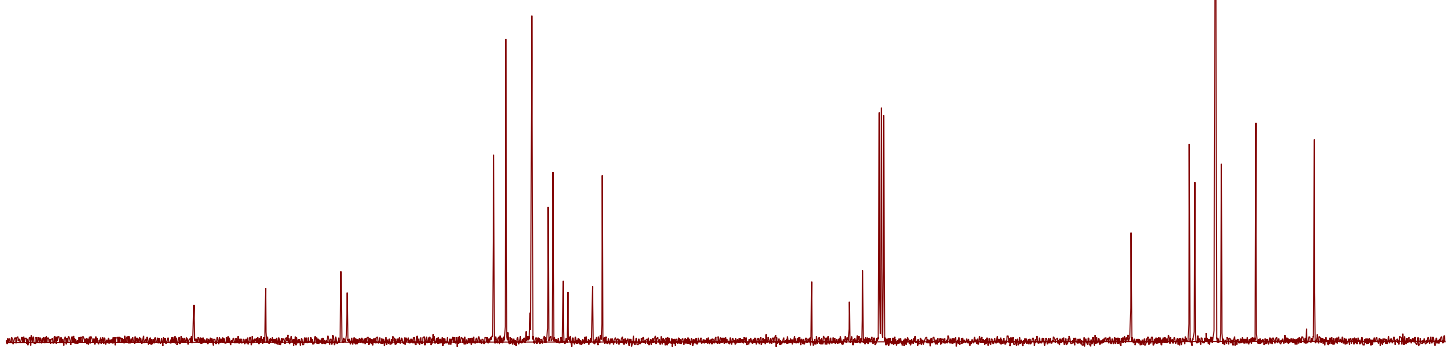

200

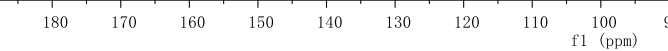


3m- ${ }^{1} \mathrm{H}$ NMR (400 MHz, CDCl 3$)$

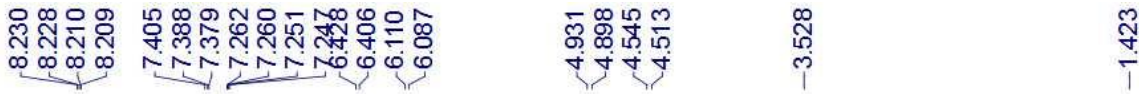<smiles>COCc1oc2ccccc2c(=O)c1C(C#Cc1ccccc1)NC(C)(C)C</smiles>

$3 m$

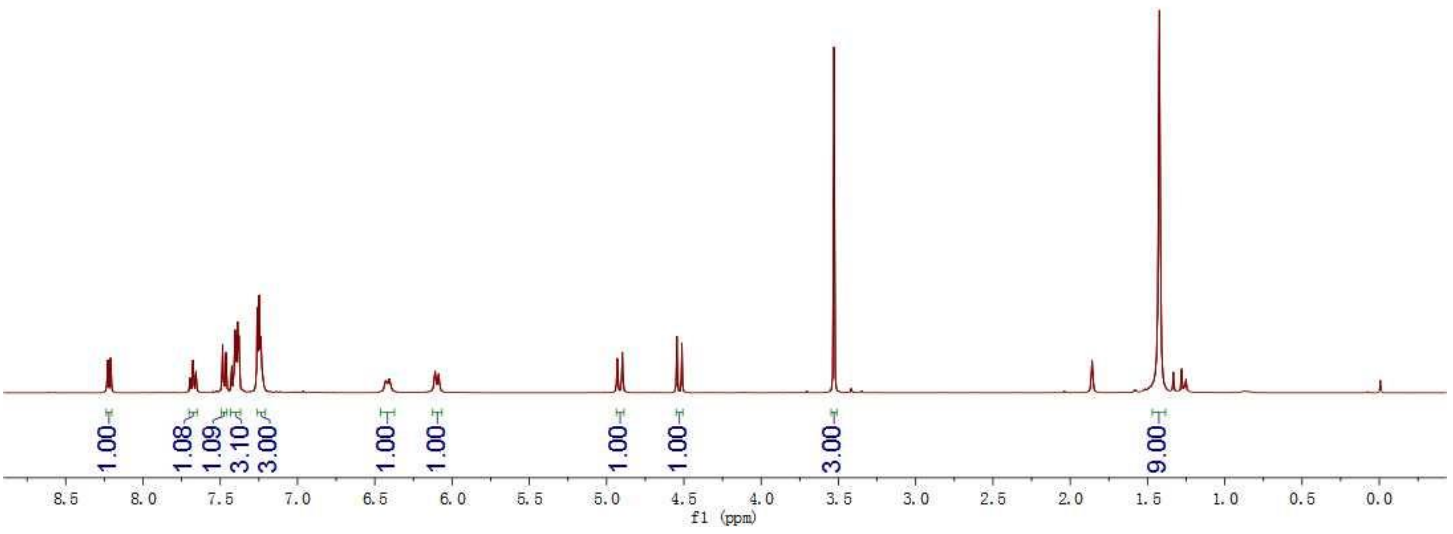

$3 \mathrm{~m}-{ }^{13} \mathrm{C}\{1 \mathrm{H}\}$ NMR (100 $\left.\mathrm{MHz}, \mathrm{CDCl}_{3}\right)$

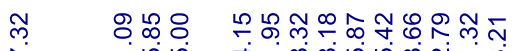

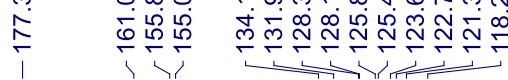

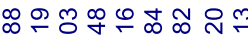

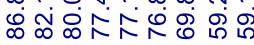

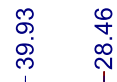<smiles>COCc1oc2ccccc2c(=O)c1C(C#Cc1ccccc1)NC(C)(C)C</smiles>

$3 m$

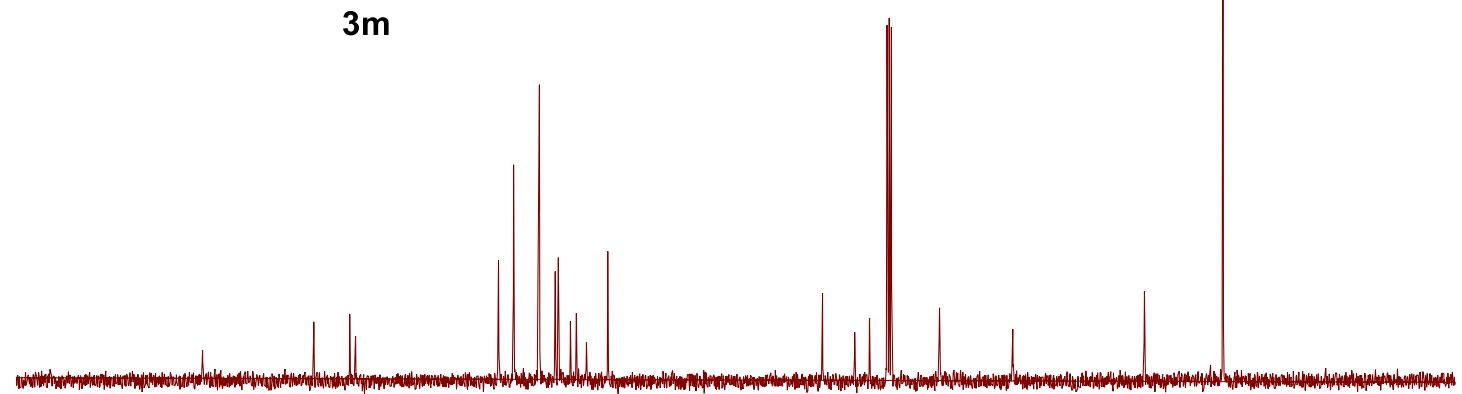

200

180

160 f1 100 (ppm) 
3n- ${ }^{1} \mathrm{H}$ NMR (400 $\left.\mathrm{MHz}, \mathrm{CDCl}_{3}\right)$

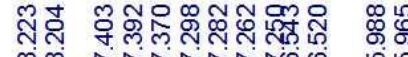

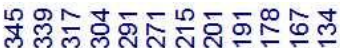

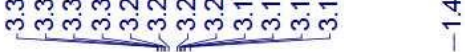<smiles>CC(C)(C)OC(=O)N[C@H](C#Cc1ccccc1)c1c(CCc2ccccc2)oc2ccccc2c1=O</smiles>

$3 n$

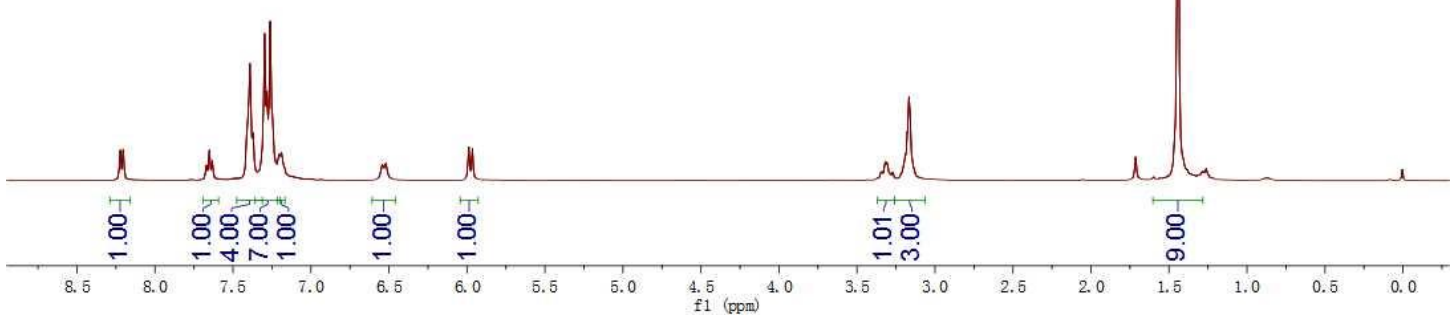

3n- ${ }^{13} \mathrm{C}\{1 \mathrm{H}\}$ NMR (150 MHz, $\left.\mathrm{CDCl}_{3}\right)$

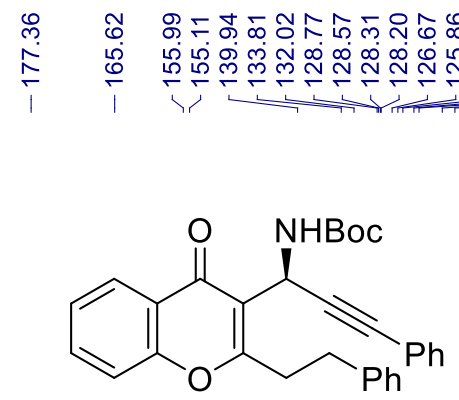

$3 n$

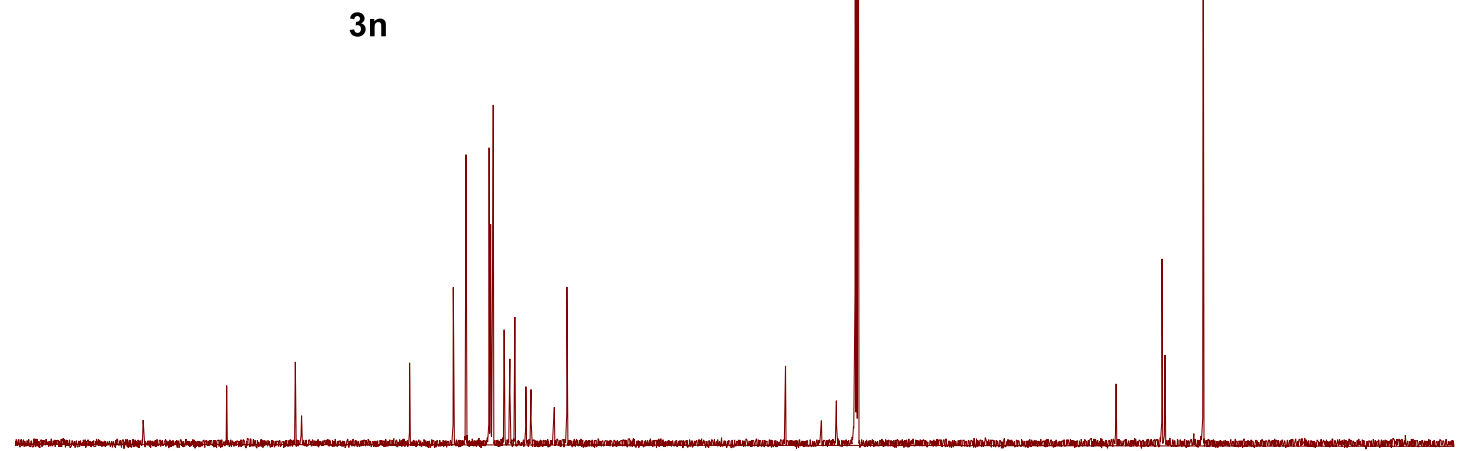


30- ${ }^{1} \mathrm{H}$ NMR (400 MHz, $\left.\mathrm{CDCl}_{3}\right)$

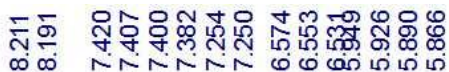<smiles>Cc1oc2ccccc2c(=O)c1C(C#Cc1ccccc1Br)NC(=O)OC(C)(C)C</smiles>

30

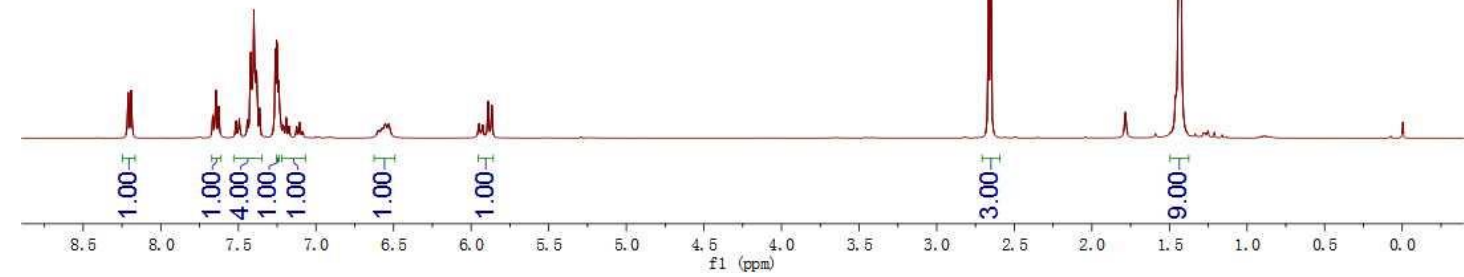

3o- ${ }^{13} \mathrm{C}\{1 \mathrm{H}\}$ NMR (100 MHz, $\left.\mathrm{CDCl}_{3}\right)$

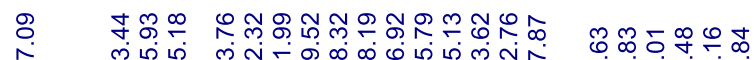

年 $\quad$<smiles>Cc1oc2ccccc2c(=O)c1C(C#Cc1ccccc1Br)NC(=O)OC(C)(C)C</smiles>

30

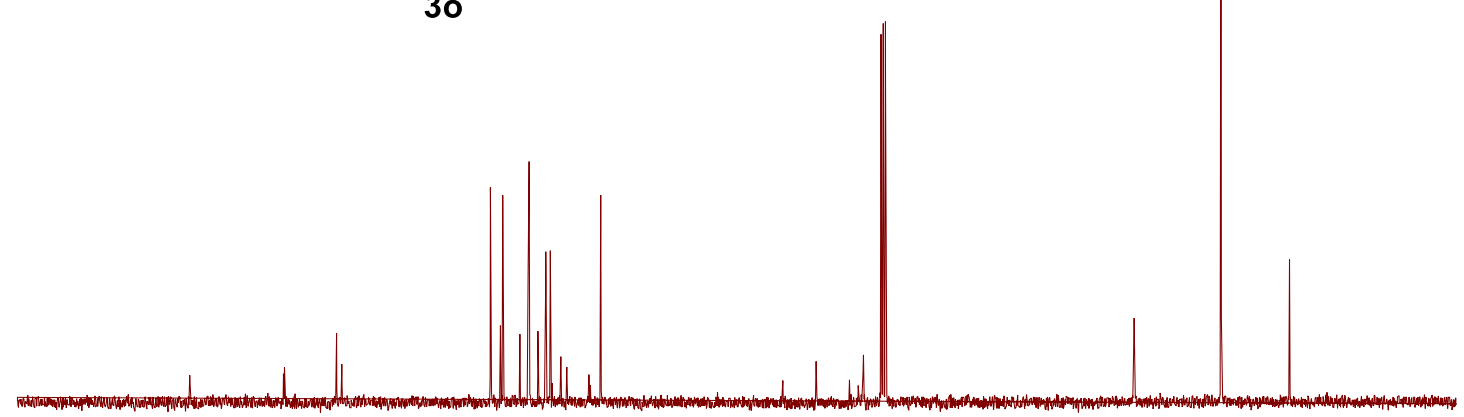

To0

180

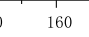

$140 \quad 130$ 
3p- ${ }^{1} \mathrm{H}$ NMR (400 MHz, CDCl3)

舟

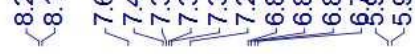

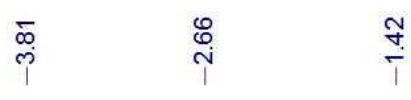<smiles>COc1ccccc1C#C[C@H](NC(=O)OC(C)(C)C)c1c(C)oc2ccccc2c1=O</smiles>

$3 p$

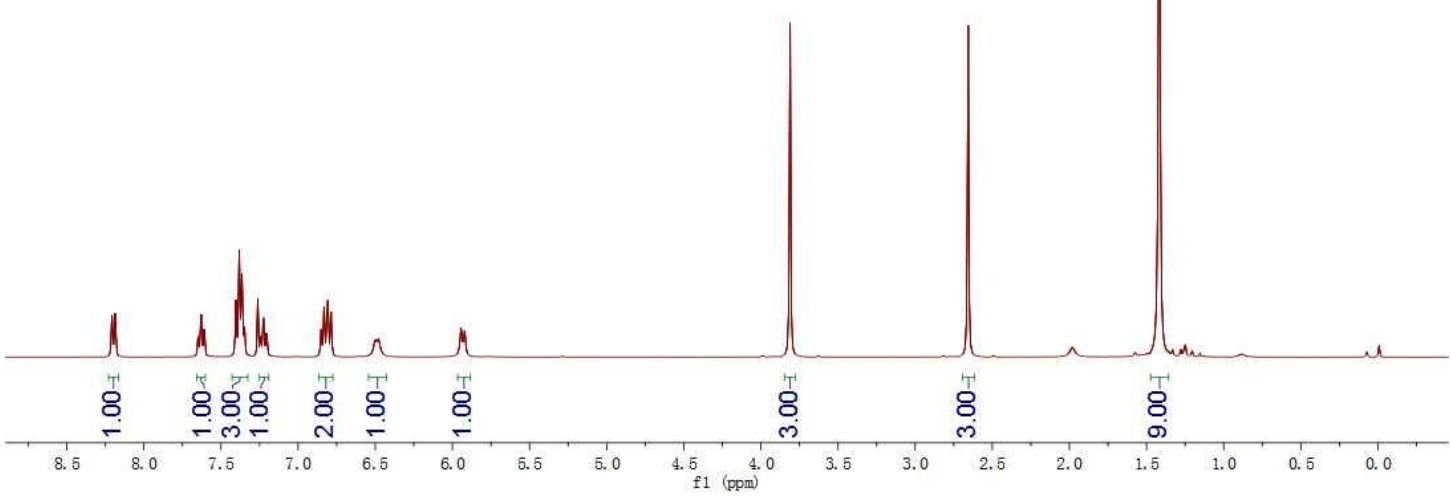

3p- ${ }^{13} \mathrm{C}\{1 \mathrm{H}\}$ NMR (100 MHz, $\left.\mathrm{CDCl}_{3}\right)$

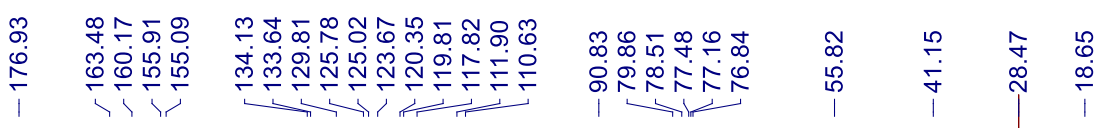<smiles>COc1ccccc1C#C[C@H](NC(=O)OC(C)(C)C)c1c(C)oc2ccccc2c1=O</smiles>

$3 p$

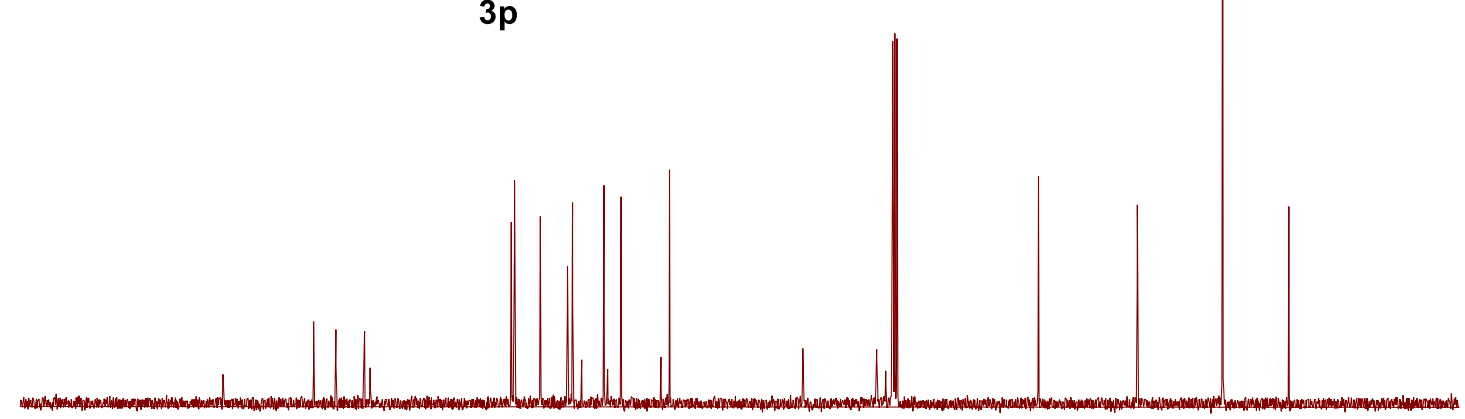

$\begin{array}{lllllllllll}200 & 190 & 180 & 170 & 160 & 150 & 140 & 130 & 120 & 110 & 100 \\ \mathrm{f} 1 & (\mathrm{ppm})\end{array}$ 
3q- ${ }^{1} \mathrm{H}$ NMR (400 MHz, CDCl3)

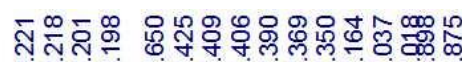

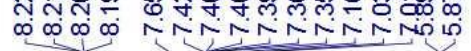

응

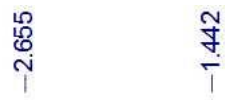<smiles>Cc1oc2ccccc2c(=O)c1C(C#Cc1cccc(OCc2ccccc2)c1)NC(C)(C)C</smiles>

$3 q$

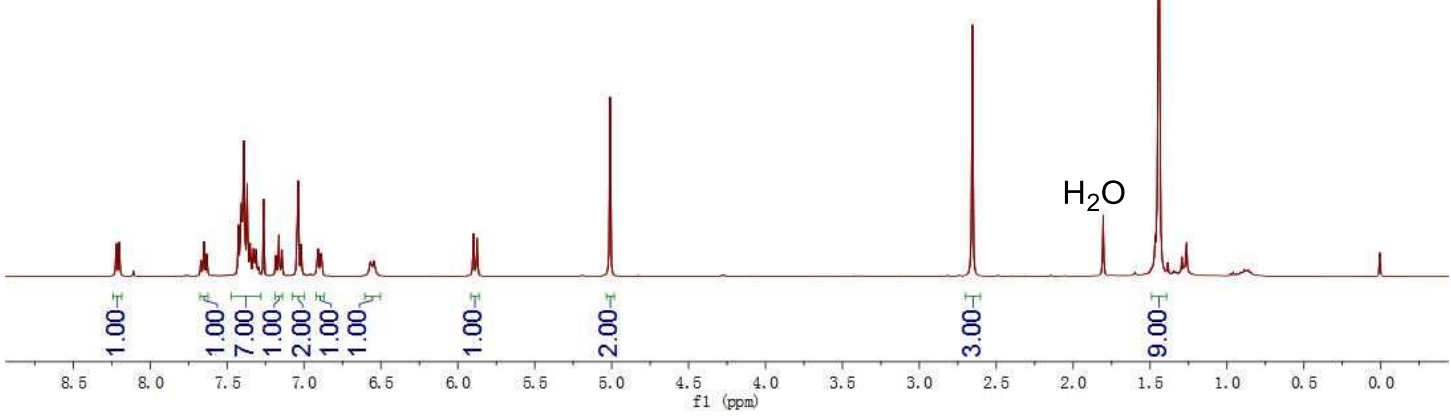

3q- ${ }^{13} \mathrm{C}\{1 \mathrm{H}\}$ NMR (150 MHz, $\left.\mathrm{CDCl}_{3}\right)$

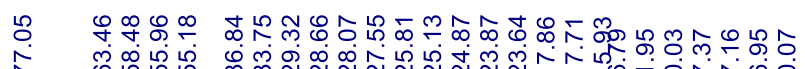

定

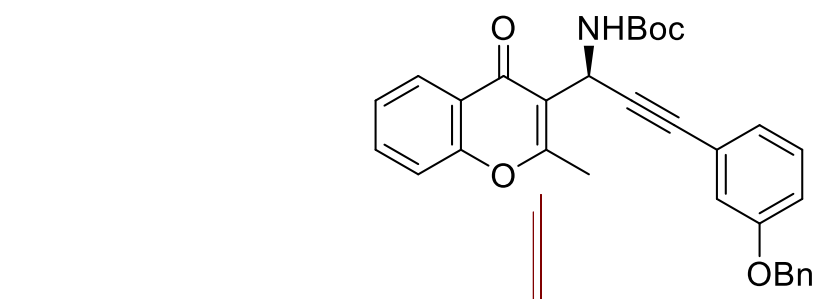

$3 q$

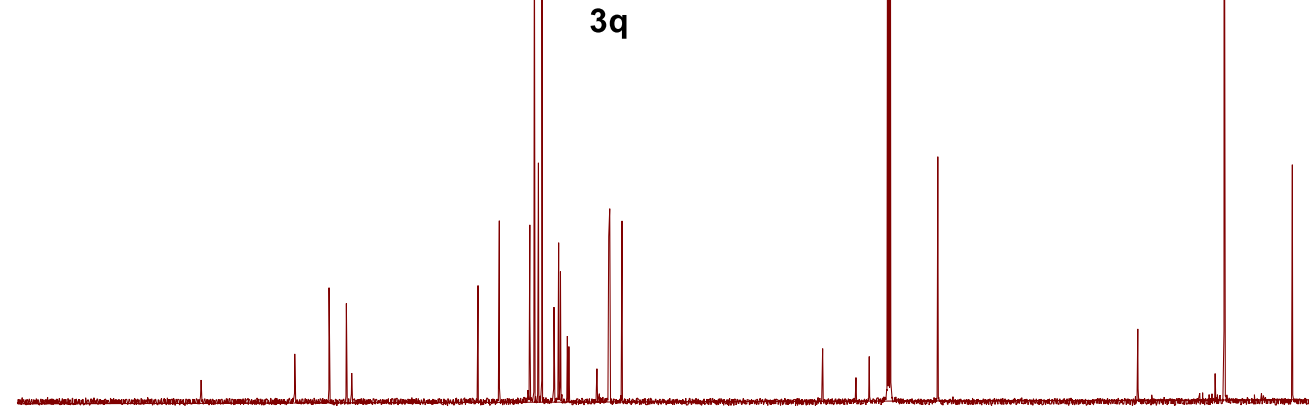

$90 \quad 180$

160

$140 \quad 130$

$20 \quad 110$

100 
3r- ${ }^{1} \mathrm{H}$ NMR (400 MHz, CDCl3)

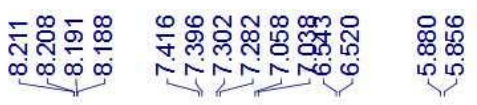

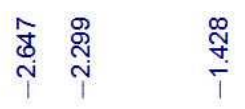<smiles>Cc1ccc(C#CC(NC(=O)OC(C)(C)C)c2c(C)oc3ccccc3c2=O)cc1</smiles>

$3 r$

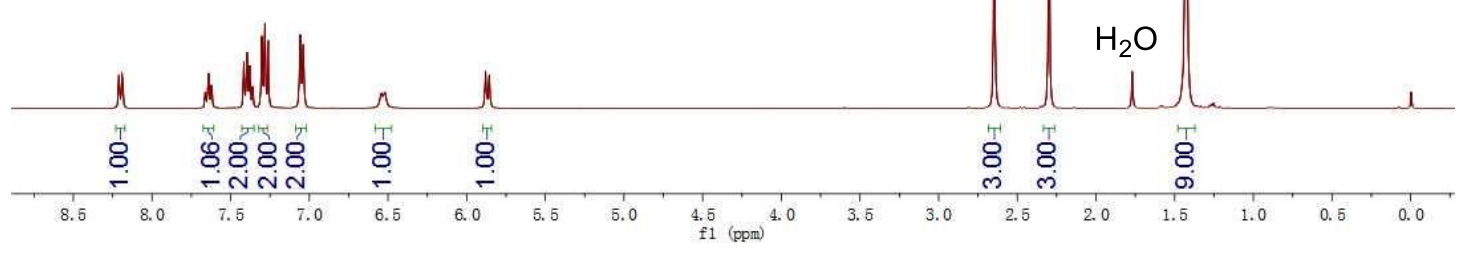

3r- ${ }^{13} \mathrm{C}\{1 \mathrm{H}\}$ NMR (100 MHz, $\left.\mathrm{CDCl}_{3}\right)$

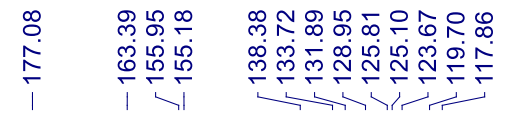

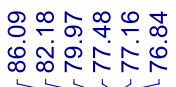
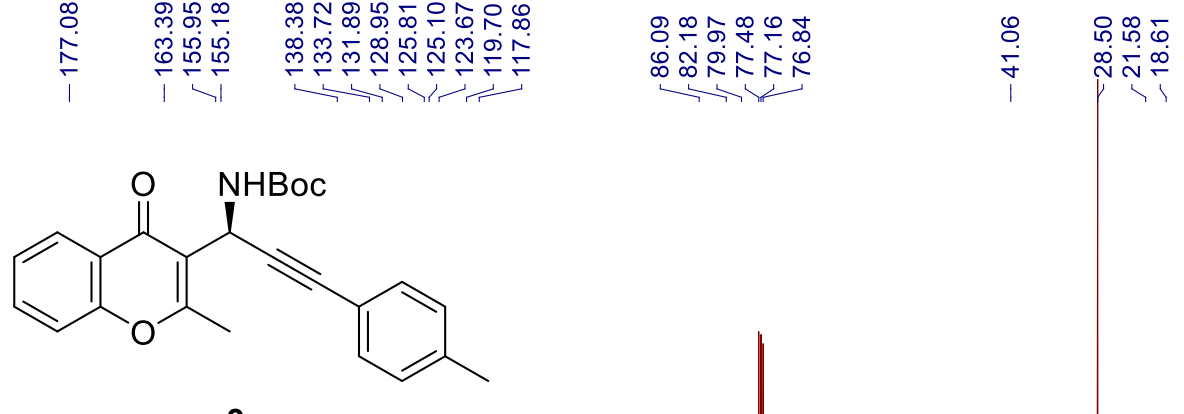

$3 r$

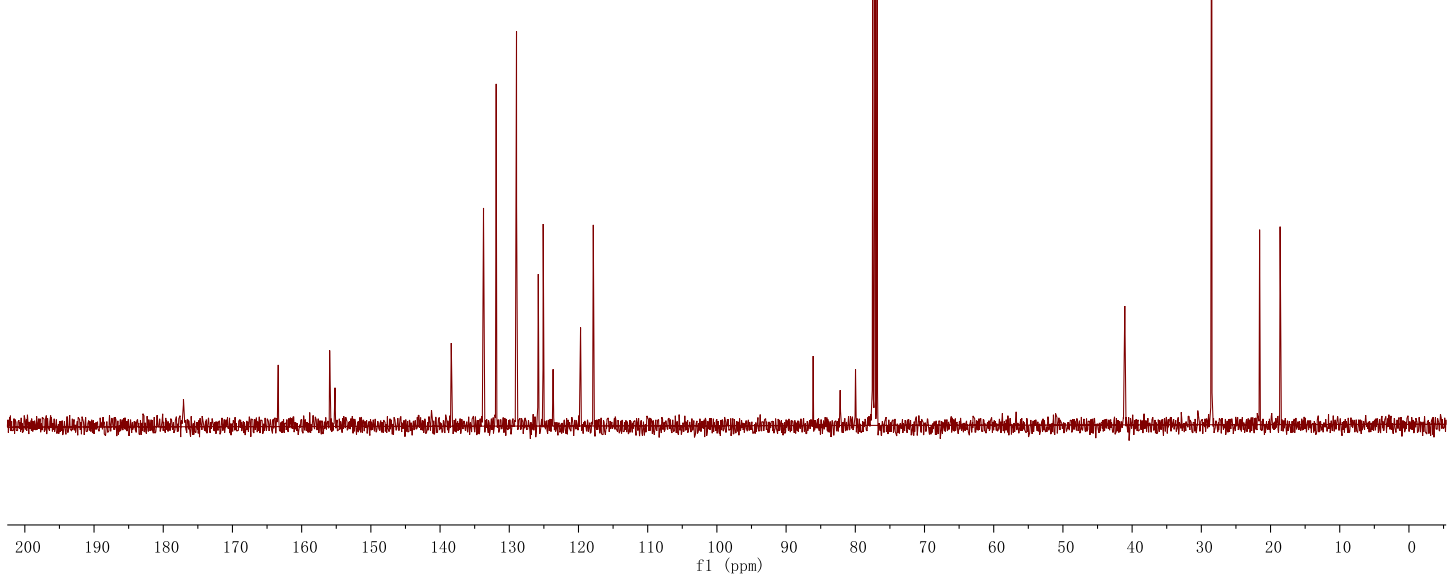

89 
3s- ${ }^{1} \mathrm{H}$ NMR (400 MHz, CDCl3)

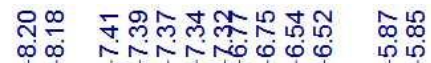

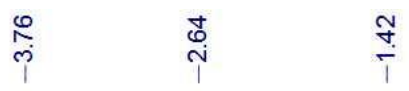<smiles>COc1ccc(C#C[C@H](NC(=O)OC(C)(C)C)c2c(C)oc3ccccc3c2=O)cc1</smiles>

$3 s$

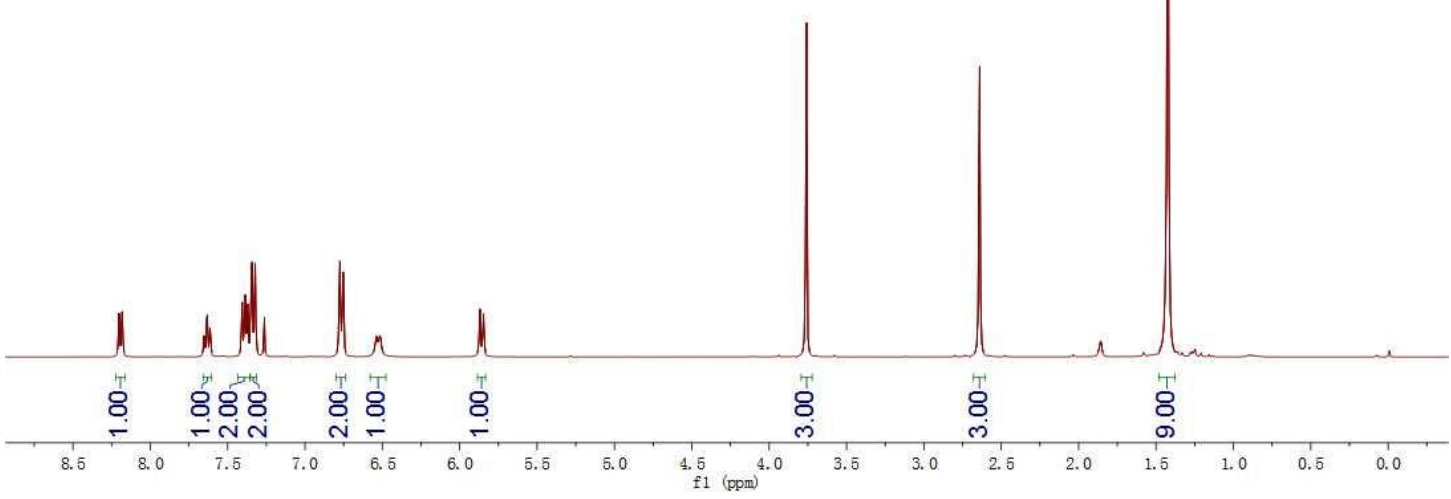

3s- ${ }^{13} \mathrm{C}\{1 \mathrm{H}\}$ NMR (100 MHz, CDCl 3$)$

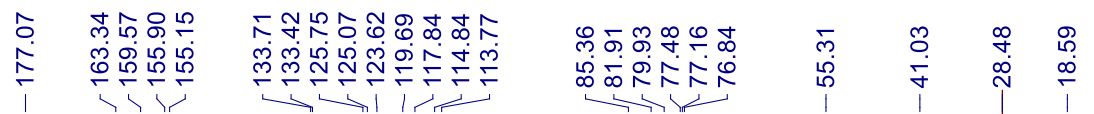<smiles>COc1ccc(C#C[C@H](NC(=O)OC(C)(C)C)c2c(C)oc3ccccc3c2=O)cc1</smiles>

$3 s$

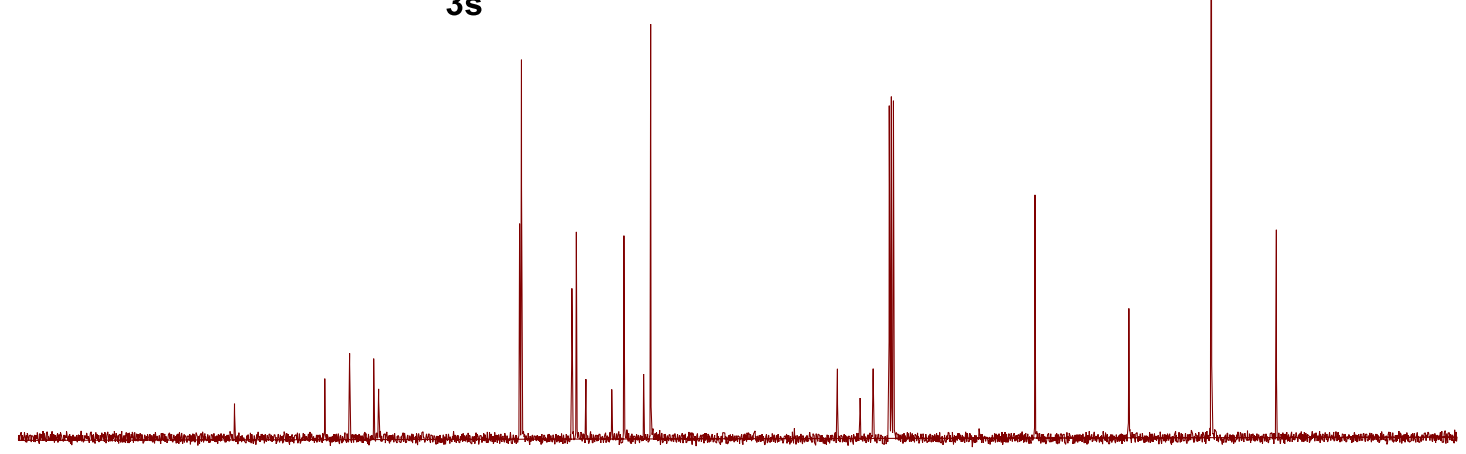

$\begin{array}{lllllllllll}200 & 190 & 180 & 170 & 160 & 150 & 140 & 130 & 120 & 110 & 100 \\ \mathrm{f} 1 & (\mathrm{ppm})\end{array}$ 
3t- ${ }^{1} \mathrm{H}$ NMR (400 $\left.\mathrm{MHz}, \mathrm{CDCl}_{3}\right)$

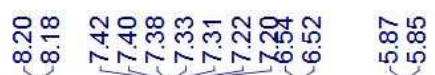

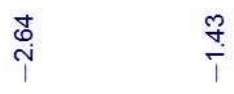<smiles>Cc1oc2ccccc2c(=O)c1[C@H](C#Cc1ccc(Cl)cc1)N[R6](=O)[O-]</smiles>

$3 t$

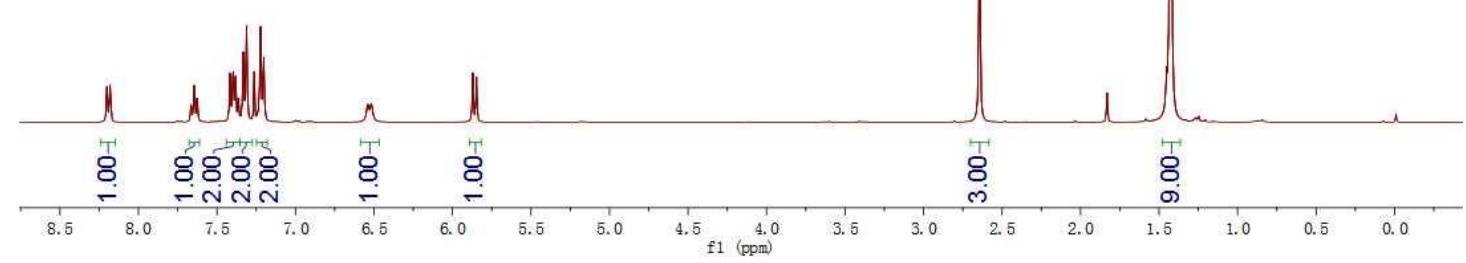

3t- ${ }^{13} \mathrm{C}\{1 \mathrm{H}\}$ NMR (100 MHz, CDCl 3$)$

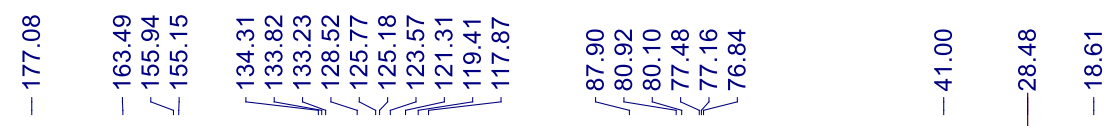<smiles>Cc1oc2ccccc2c(=O)c1C(C#Cc1ccc(Cl)cc1)NC(=O)OC(C)(C)C</smiles>

$3 t$

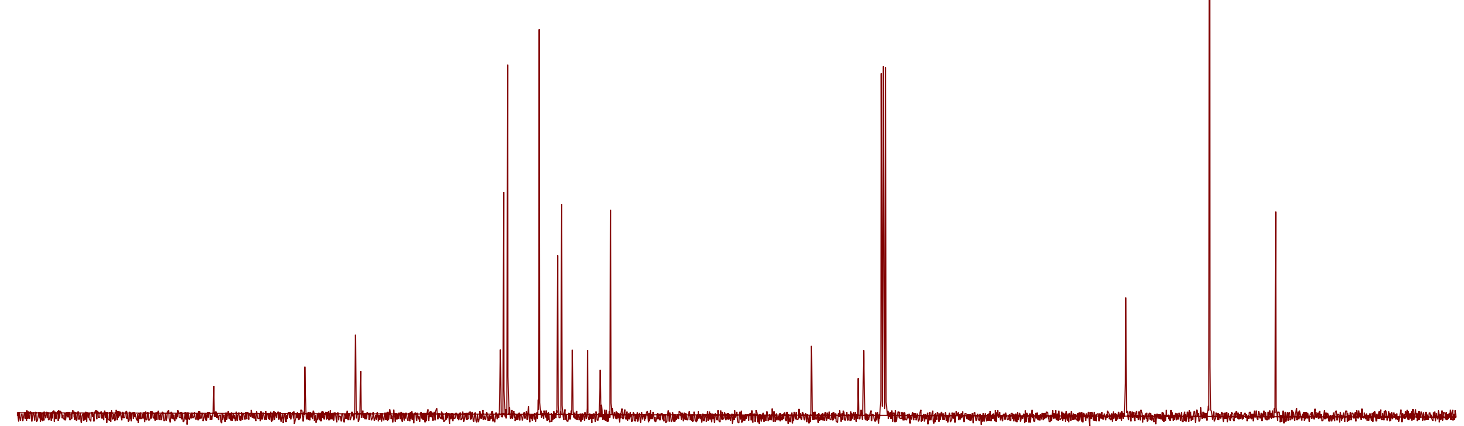


3u- ${ }^{1} \mathrm{H}$ NMR (400 $\left.\mathrm{MHz}, \mathrm{CDCl}_{3}\right)$

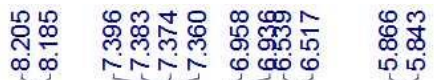<smiles>Cc1oc2ccccc2c(=O)c1C(C#Cc1ccc(F)cc1)NC(=O)OC(C)(C)C</smiles>

$3 u$

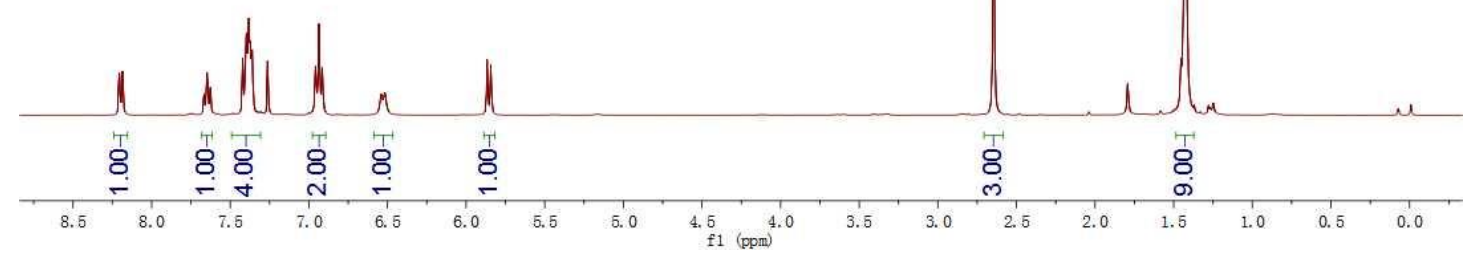

3u- ${ }^{13} \mathrm{C}\{1 \mathrm{H}\}$ NMR (150 MHz, $\left.\mathrm{CDCl}_{3}\right)$

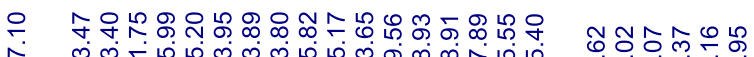

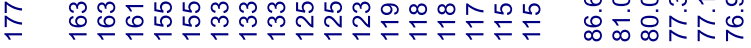<smiles>Cc1oc2ccccc2c(=O)c1C(C#Cc1ccc(F)cc1)NC(=O)OC(C)(C)C</smiles>

$3 u$

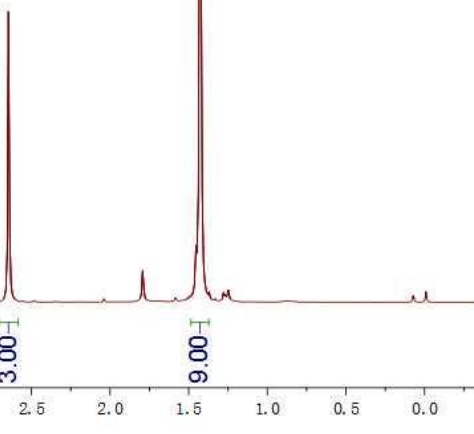


3v- ${ }^{1} \mathrm{H}$ NMR (400 MHz, CDCl3)

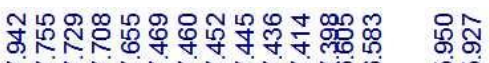

ñNañNañon

ח
$\stackrel{0}{0}$<smiles>Cc1oc2ccccc2c(=O)c1[C@H](C#Cc1ccc2ccccc2c1)NC(=O)O</smiles>

$3 \mathbf{v}$

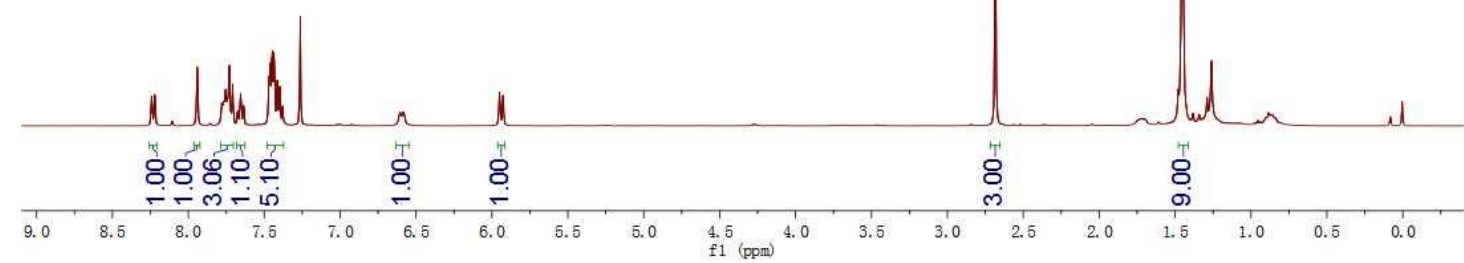

$3 v-{ }^{13} \mathrm{C}\{1 \mathrm{H}\}$ NMR (150 $\left.\mathrm{MHz}, \mathrm{CDCl}_{3}\right)$

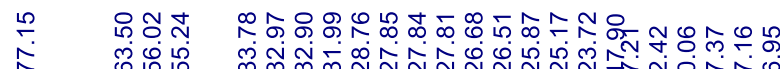

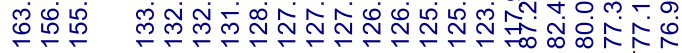

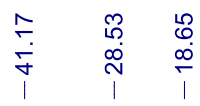<smiles>Cc1oc2ccccc2c(=O)c1[C@H](C#Cc1ccc2ccccc2c1)C(=O)OC(C)(C)C</smiles>

3v

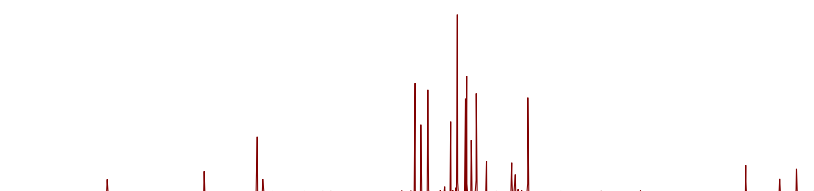

$190 \quad 180$

170

160

f1 $\begin{gathered}90 \\ (\mathrm{ppm})\end{gathered}$ 
3x- ${ }^{1} \mathrm{H}$ NMR (400 MHz, $\mathrm{CDCl}_{3}$ )

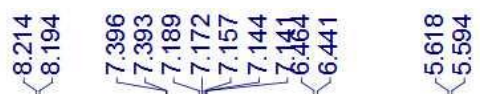

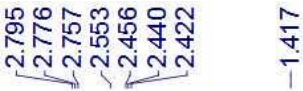<smiles>Cc1oc2ccccc2c(=O)c1C(C#CCCc1ccccc1)NC(C)(C)C</smiles>

$3 x$

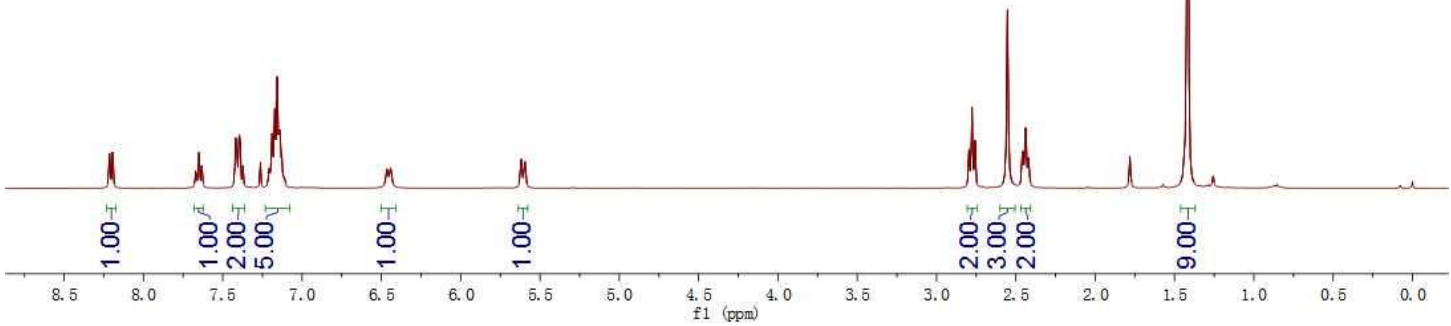

3X- ${ }^{13} \mathrm{C}\{1 \mathrm{H}\}$ NMR (100 MHz, CDCl $)$

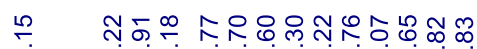

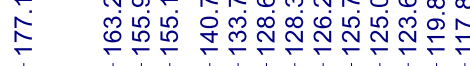

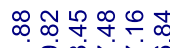

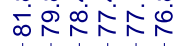

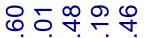

促

守少市<smiles>CO[R6](=O)N[C@@H](C#CCCc1ccccc1)c1c(C)oc2ccccc2c1=O</smiles>

$3 x$

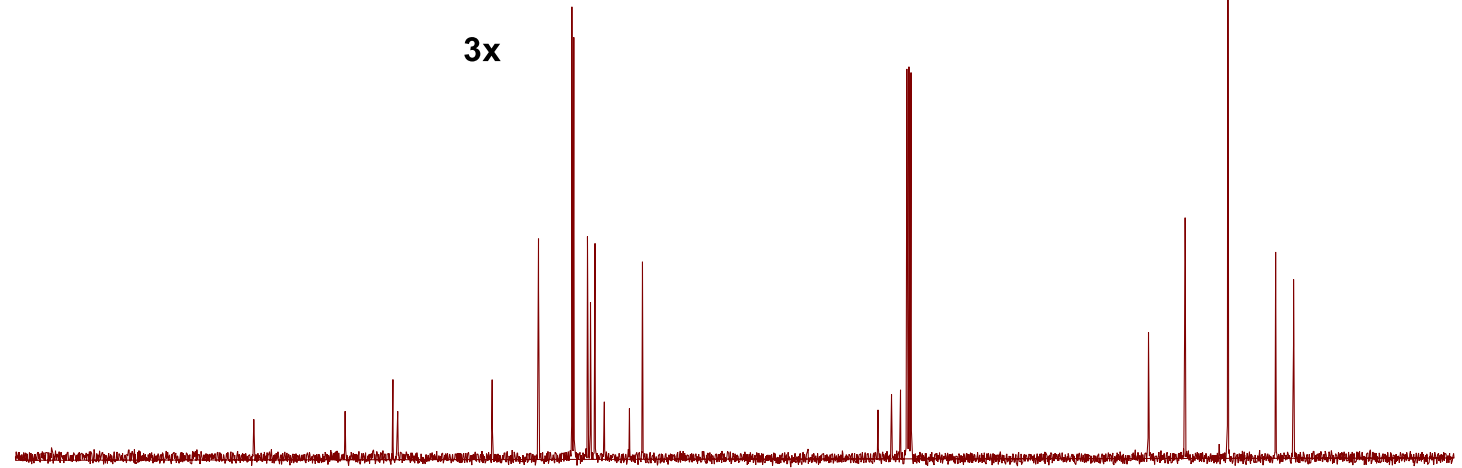


4a- ${ }^{1} \mathrm{H}$ NMR (400 MHz, CDCl3)

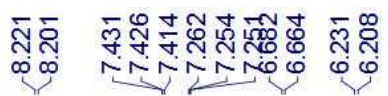

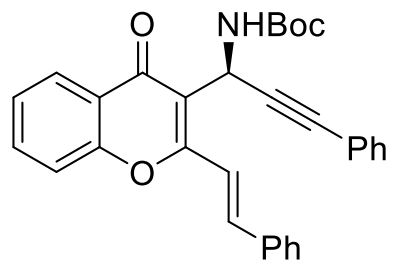

$4 a$

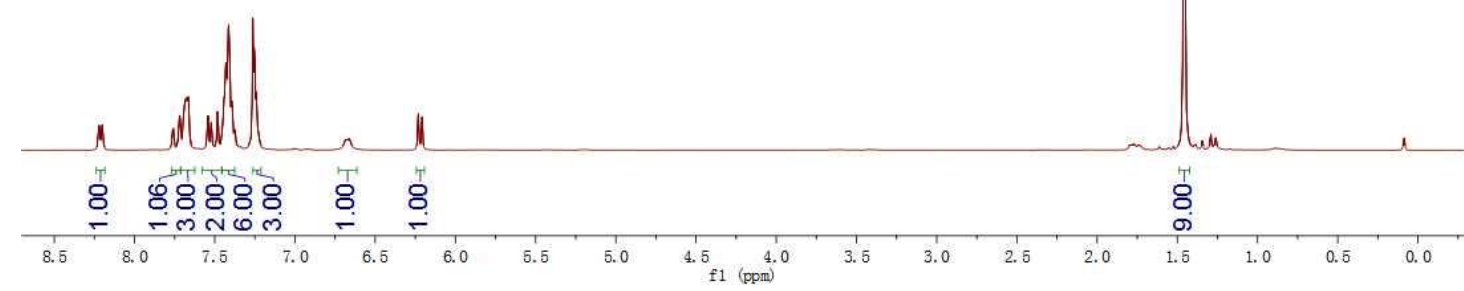

4a- ${ }^{13} \mathrm{C}\{1 \mathrm{H}\}$ NMR (150 MHz, CDCl 3$)$

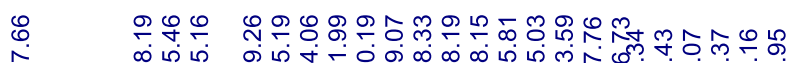

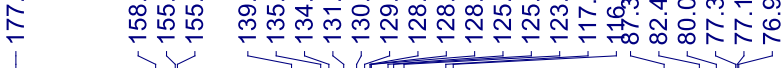<smiles>CC(C)(C)OC(=O)N[C@@H](C#Cc1ccccc1)c1c(/C=C/c2ccccc2)oc2ccccc2c1=O</smiles>

$4 a$

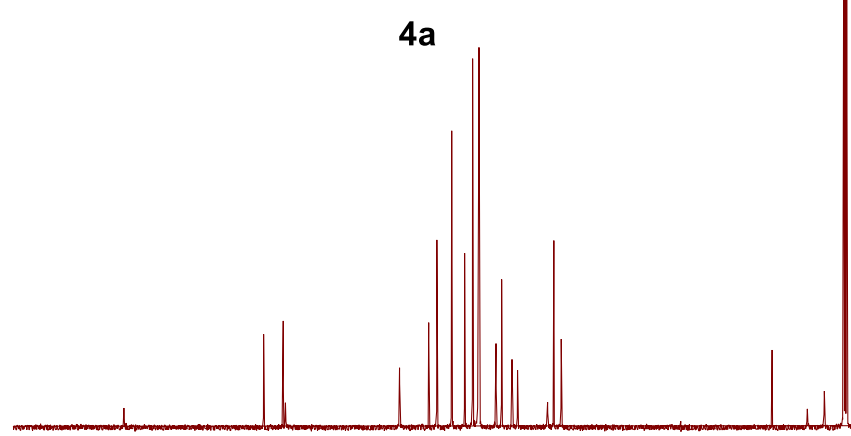

$190 \quad 180 \quad 170$

${ }^{100}{ }_{\mathrm{f} 1} \begin{array}{r}90 \\ (\mathrm{ppm})\end{array}$ 
4b- ${ }^{1} \mathrm{H}$ NMR (400 $\left.\mathrm{MHz}, \mathrm{CDCl}_{3}\right)$

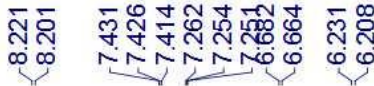

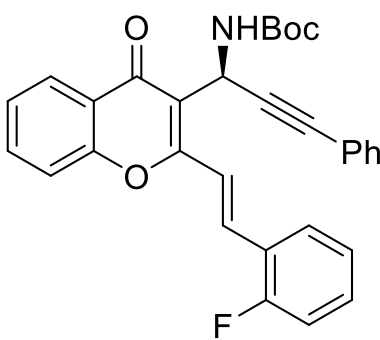

$4 b$

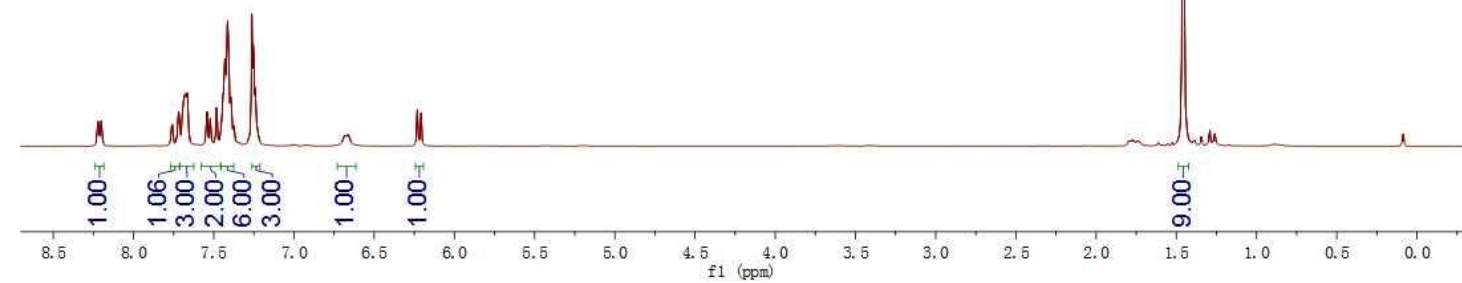

4b- ${ }^{13} \mathrm{C}\{1 \mathrm{H}\}$ NMR (150 MHz, CDCl 3$)$

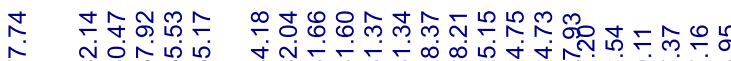

今

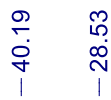<smiles>CC(C)(C)OC(=O)C[C@H](C#Cc1ccccc1)c1c(/C=C/c2ccccc2F)oc2ccccc2c1=O</smiles>

4b

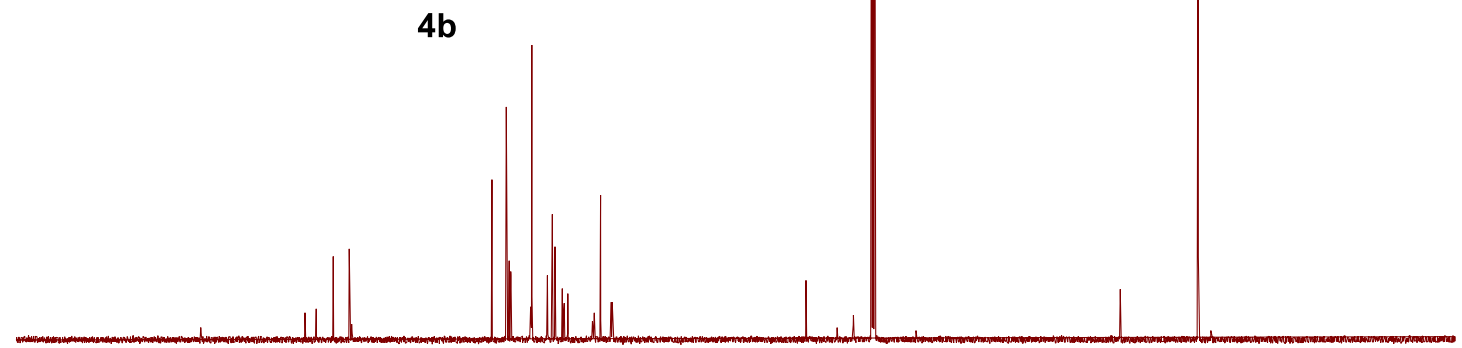

$200 \quad 190$

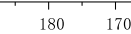

10090 
4c- ${ }^{1} \mathrm{H}$ NMR (400 MHz, CDCl3)

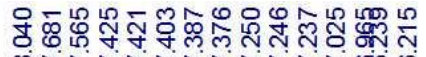

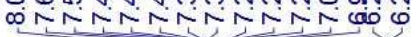

怘<smiles>COC(=O)[C@@H](C#Cc1ccccc1)c1c(/C=C/c2ccccc2OC)oc2ccccc2c1=O</smiles>

4c

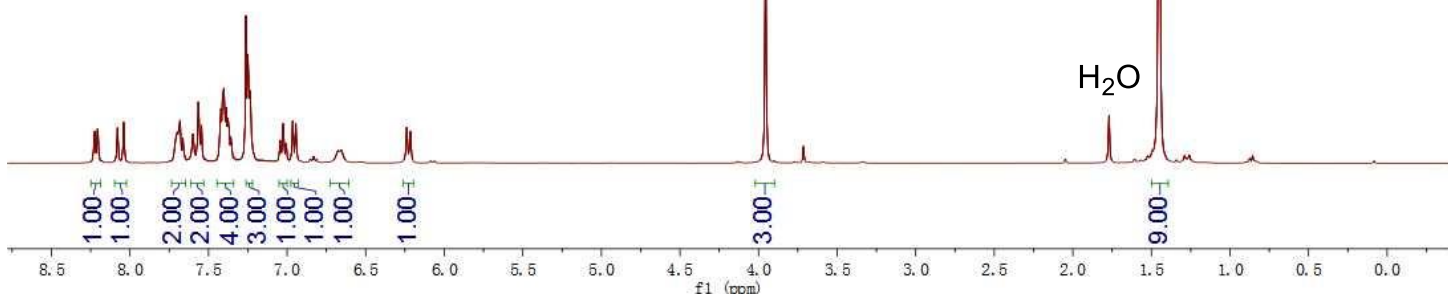

4c- ${ }^{13} \mathrm{C}\{1 \mathrm{H}\}$ NMR (100 $\left.\mathrm{MHz}, \mathrm{CDCl}_{3}\right)$

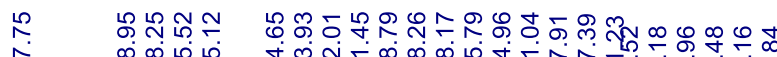

I

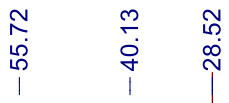

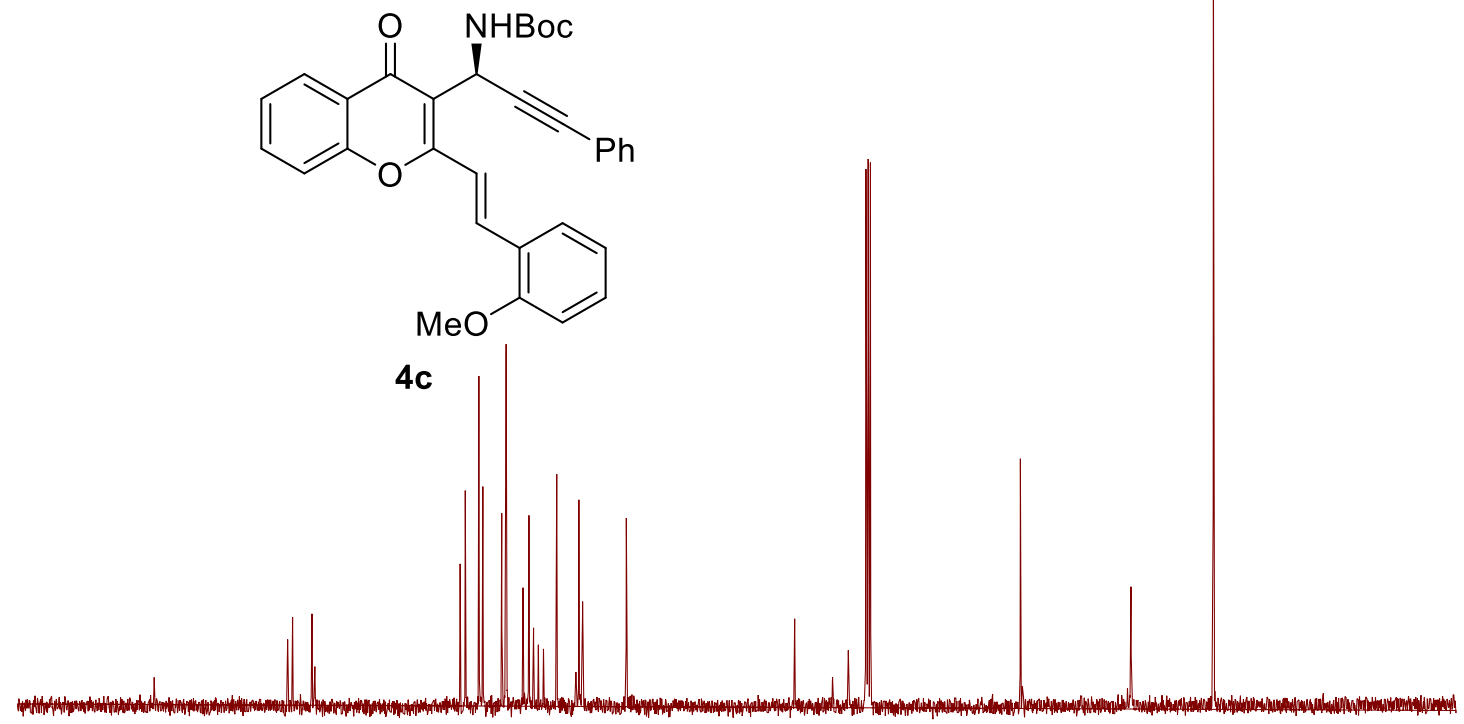

$4 c$

$\stackrel{\substack{i \\ \hdashline}}{1}$

$\begin{array}{llllllllll}190 & 180 & 170 & 160 & 150 & 140 & 130 & 120 & 110 & \left.\begin{array}{c}100 \\ \text { f1 } 1(\mathrm{ppm})\end{array}\right)\end{array}$ 
4d- ${ }^{1} \mathrm{H}$ NMR (400 $\left.\mathrm{MHz}, \mathrm{CDCl}_{3}\right)$

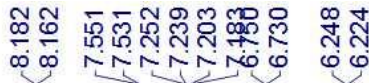

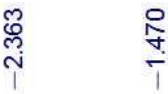

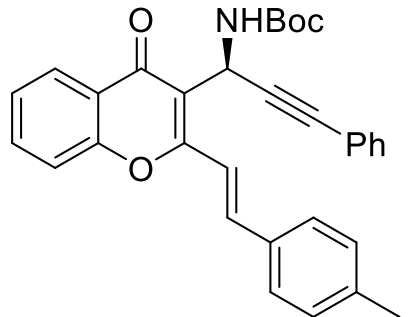

$4 d$

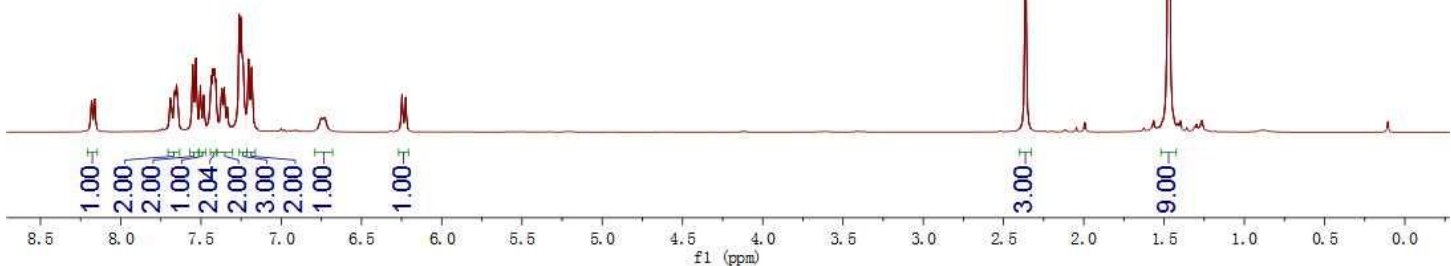

4d- ${ }^{13} \mathrm{C}\{1 \mathrm{H}\}$ NMR (150 $\left.\mathrm{MHz}, \mathrm{CDCl}_{3}\right)$

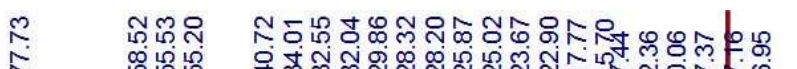

I

ה<smiles>Cc1ccc(/C=C/c2oc3ccccc3c(=O)c2[C@H](C#Cc2ccccc2)NC(C)(C)C)cc1</smiles>

4d
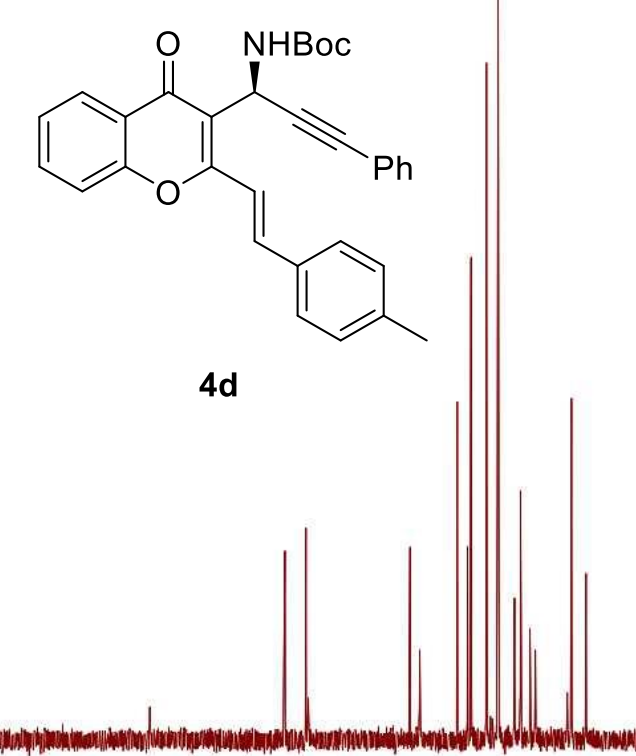

$190 \quad 180$

$170 \quad 160$

$150 \quad 1$

${ }_{f 1(\mathrm{ppm})}^{100} 90$ 
4e- ${ }^{1} \mathrm{H}$ NMR (400 MHz, $\left.\mathrm{CDCl}_{3}\right)$

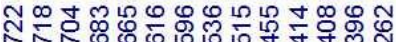<smiles>N[C@@H](C(=O)OCc1ccccc1)c1c(/C=C/c2ccc(Cl)cc2)oc2ccccc2c1=O</smiles>

$4 e$

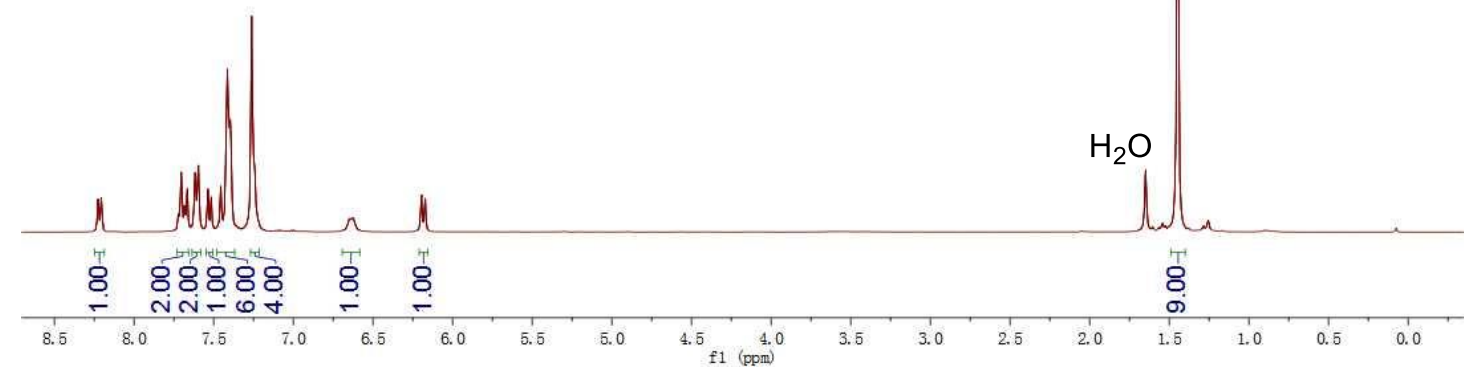

4e- ${ }^{13} \mathrm{C}\{1 \mathrm{H}\}$ NMR (150 MHz, $\left.\mathrm{CDCl}_{3}\right)$

œ

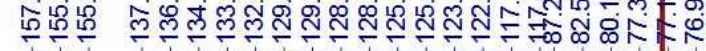

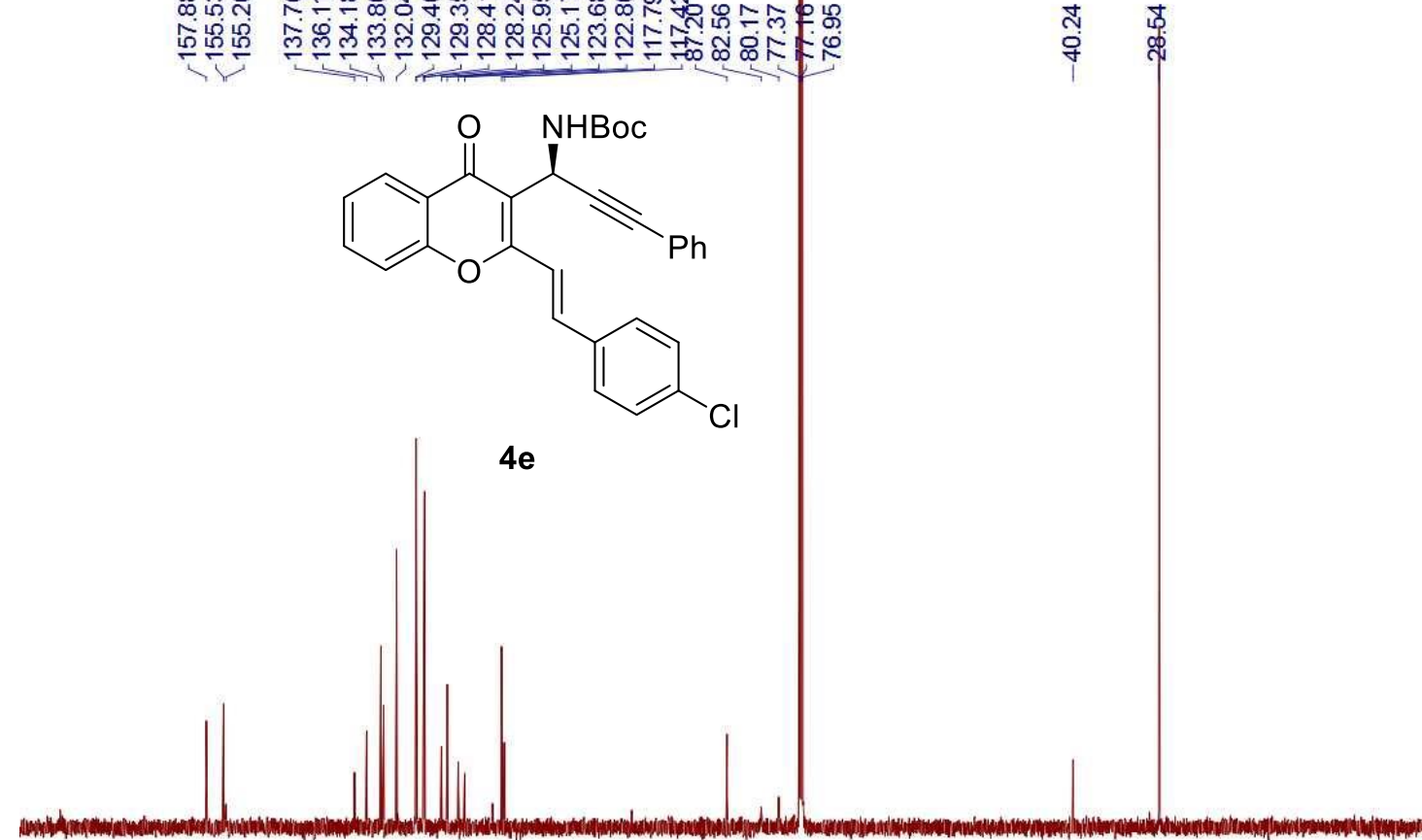

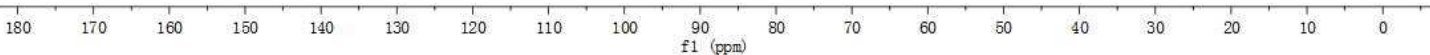


4f- ${ }^{1} \mathrm{H}$ NMR (400 MHz, $\left.\mathrm{CDCl}_{3}\right)$

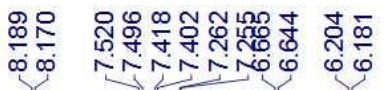

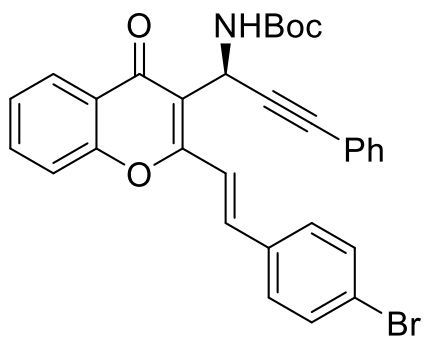

$4 f$

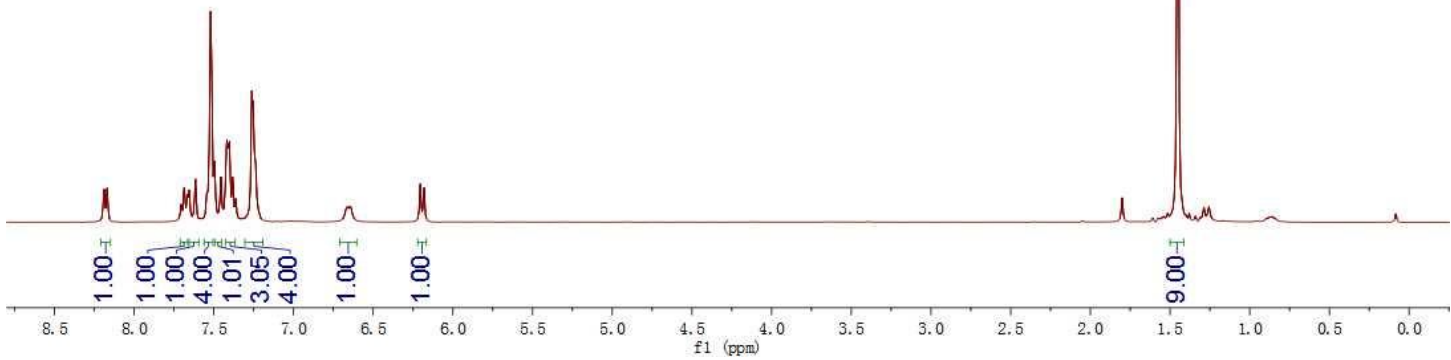

4f- ${ }^{13} \mathrm{C}\{1 \mathrm{H}\}$ NMR (150 MHz, $\left.\mathrm{CDCl}_{3}\right)$

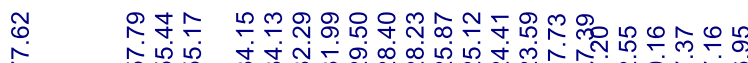

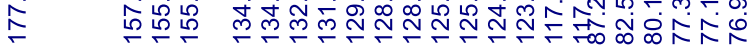

i
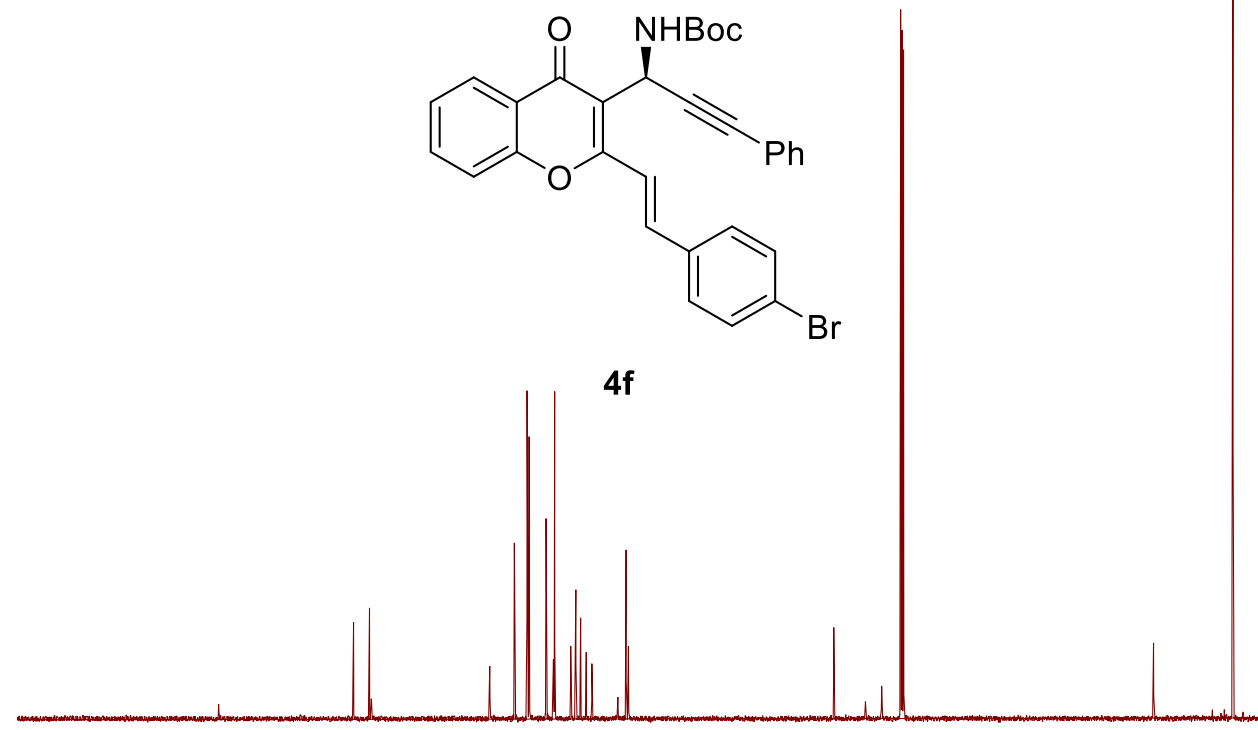

200

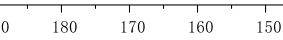

${ }_{140}^{1} \quad 130$

$\begin{array}{lll}110 & 100 \\ & & 1\end{array}$ 
4g- ${ }^{1} \mathrm{H}$ NMR (400 $\left.\mathrm{MHz}, \mathrm{CDCl}_{3}\right)$

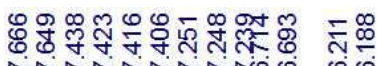

NNNNNNNNOO

莳<smiles>Cc1ccc2oc(/C=C/c3ccccc3)c([C@H](C#Cc3ccccc3)NC(=O)OC(C)(C)C)c(=O)c2c1</smiles>

$4 g$

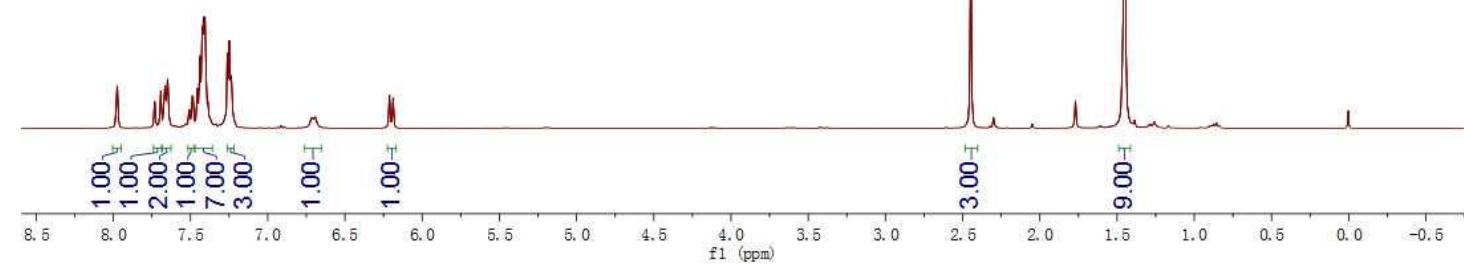

4g- ${ }^{13} \mathrm{C}\{1 \mathrm{H}\} \mathrm{NMR}\left(150 \mathrm{MHz}, \mathrm{CDCl}_{3}\right)$

急

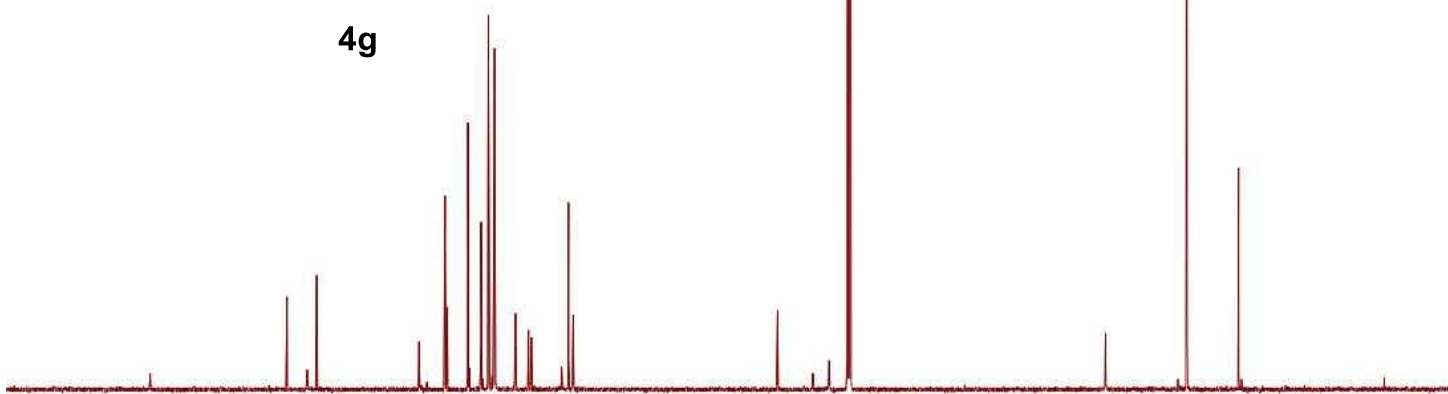

190

170

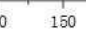

100
$\mathrm{f} 1(\mathrm{ppm})$ 
4h- ${ }^{1} \mathrm{H}$ NMR (400 MHz, CDCl3)

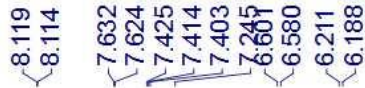<smiles>CC(C)(C)OC(=O)N[C@@H](C#Cc1ccccc1)c1c(/C=C/c2ccccc2)oc2ccc(Cl)cc2c1=O</smiles>

$4 \mathrm{~h}$

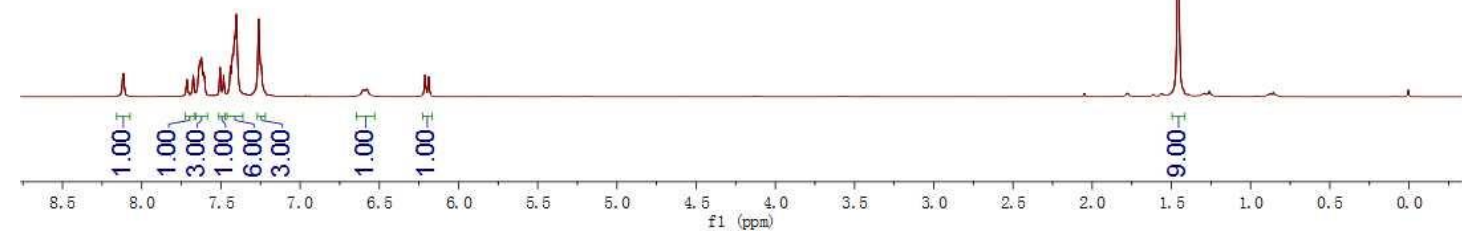

4h- ${ }^{13} \mathrm{C}\{1 \mathrm{H}\}$ NMR (150 MHz, CDCl $)$

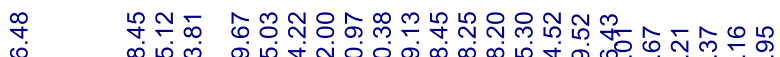

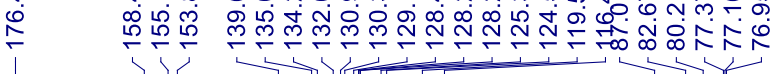

$\mathrm{Cl}$<smiles>Cc1ccc2oc(/C=C/c3ccccc3)c([C@H](C#Cc3ccccc3)N[R6](=O)[O-])c(=O)c2c1</smiles>

$4 \mathrm{~h}$

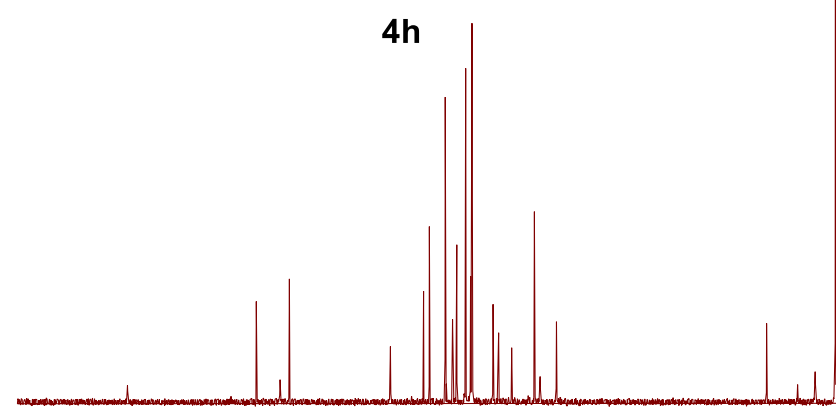

$190 \quad 180$

$100 \quad 90$ 
4i- ${ }^{1} \mathrm{H}$ NMR (400 MHz, CDCl3)

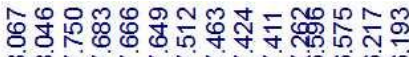

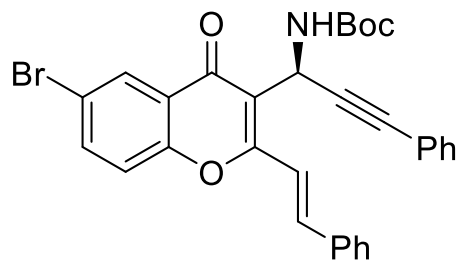

$4 \mathrm{i}$

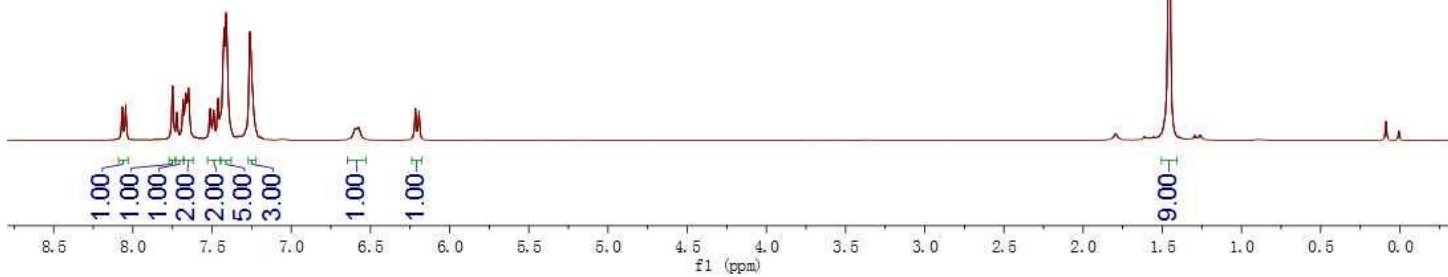

4i- ${ }^{13} \mathrm{C}\{1 \mathrm{H}\}$ NMR (150 $\left.\mathrm{MHz}, \mathrm{CDCl}_{3}\right)$

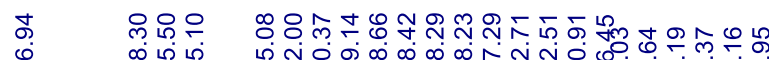

$\stackrel{0}{\circ} \quad \stackrel{0}{0}$

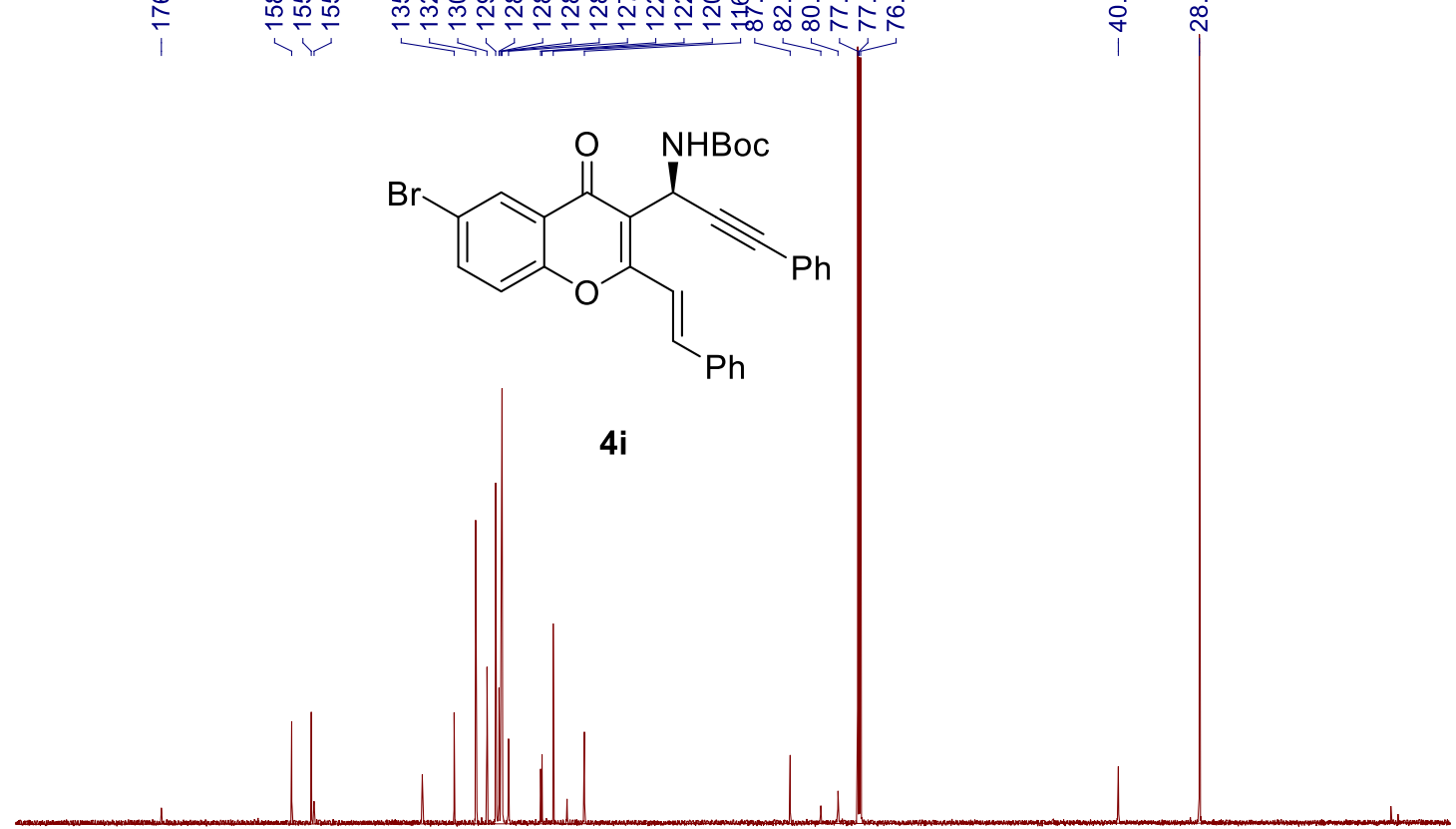

190

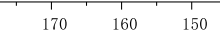

f1 (ppm) 
4j- ${ }^{1} \mathrm{H}$ NMR (400 MHz, $\left.\mathrm{CDCl}_{3}\right)$

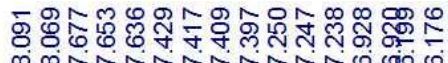

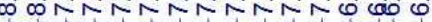

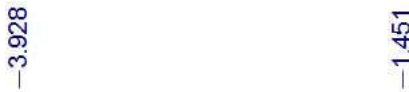<smiles>COc1ccc2c(=O)c([C@H](C#Cc3ccccc3)NC(=O)OCc3ccccc3)c(/C=C/c3ccccc3)oc2c1</smiles>

$4 \mathbf{j}$

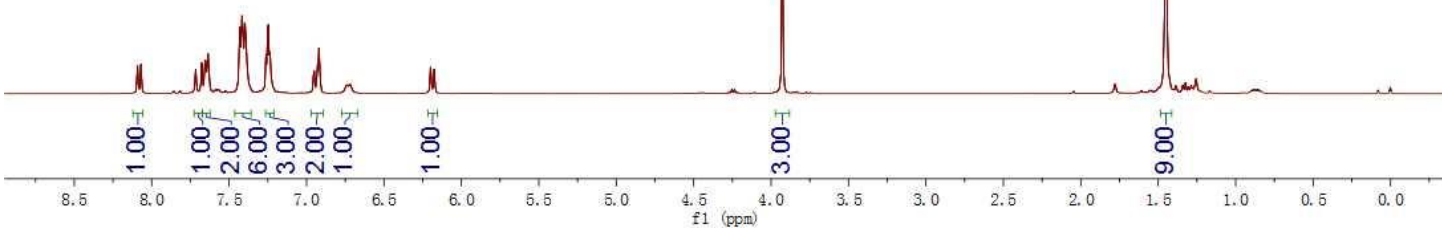

4j- ${ }^{13} \mathrm{C}\{1 \mathrm{H}\}$ NMR (150 MHz, CDCl $)$

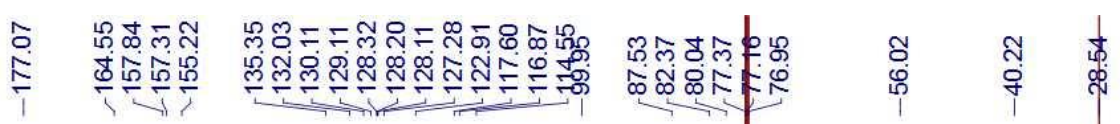

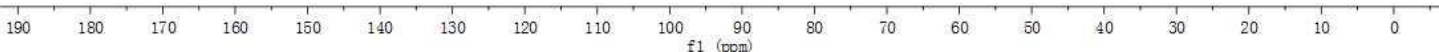


4k- ${ }^{1} \mathrm{H}$ NMR (400 MHz, CDCl3)

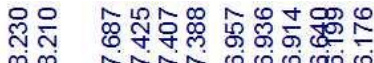

$\infty$ TNN

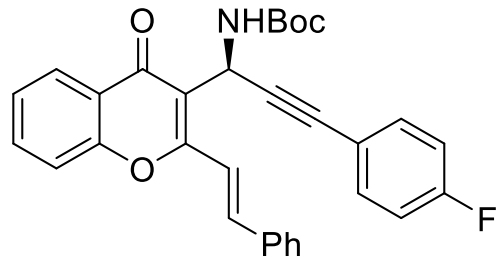

$4 k$

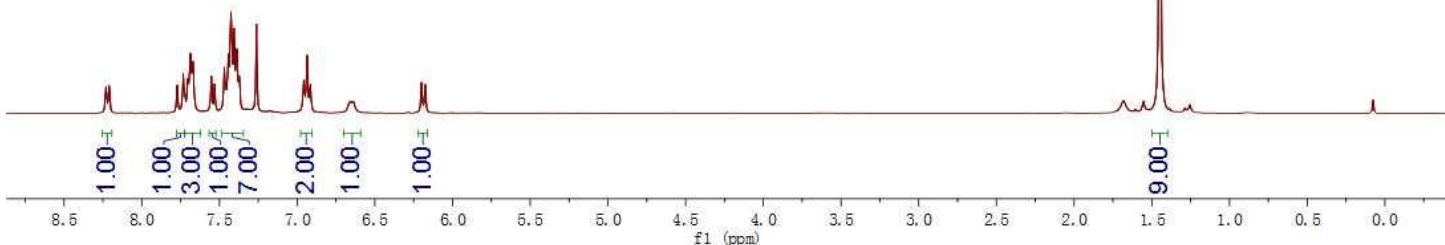

4k- ${ }^{13} \mathrm{C}\{1 \mathrm{H}\}$ NMR (150 MHz, $\left.\mathrm{CDCl}_{3}\right)$

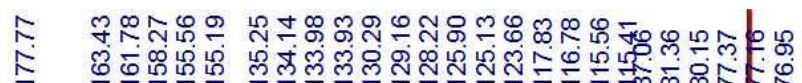

I<smiles>O=C(O)[C@H](C#Cc1ccc(F)cc1)c1c(/C=C/c2ccccc2)oc2ccccc2c1=O</smiles>
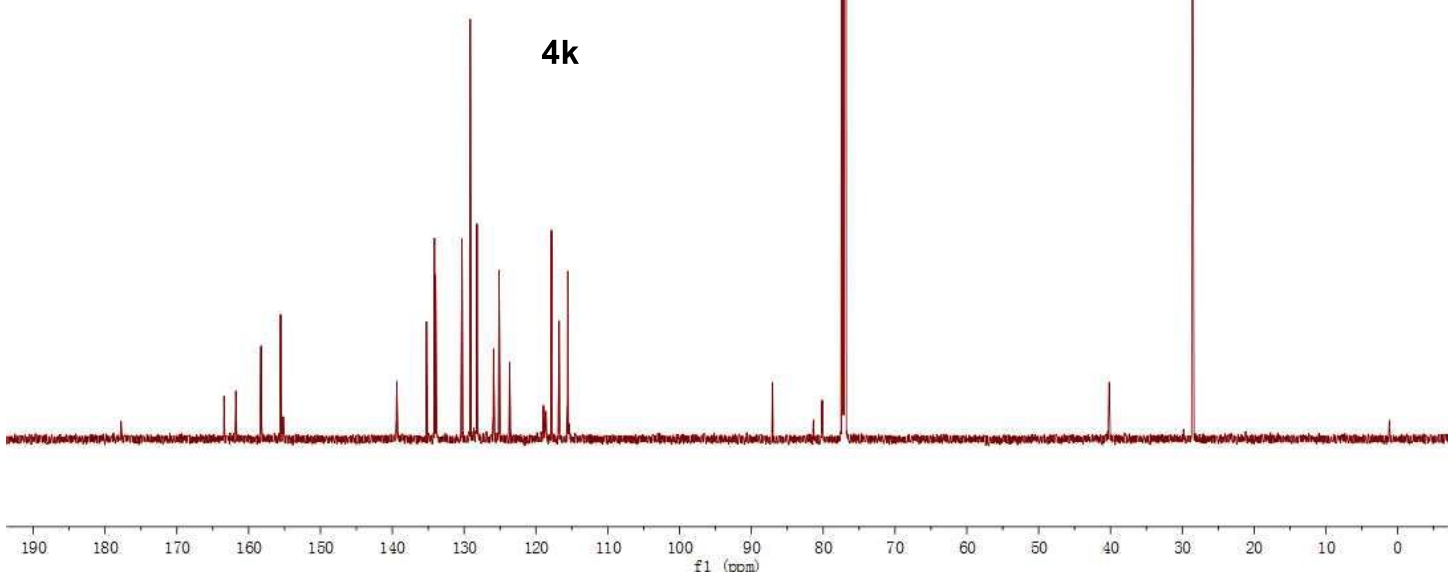


\section{4l- ${ }^{1} \mathrm{H}$ NMR (400 $\left.\mathrm{MHz}, \mathrm{CDCl}_{3}\right)$}

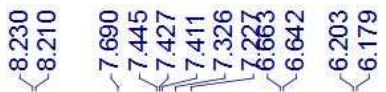

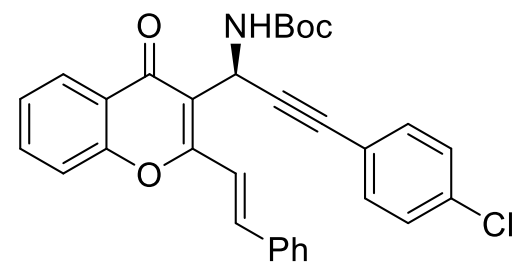

41

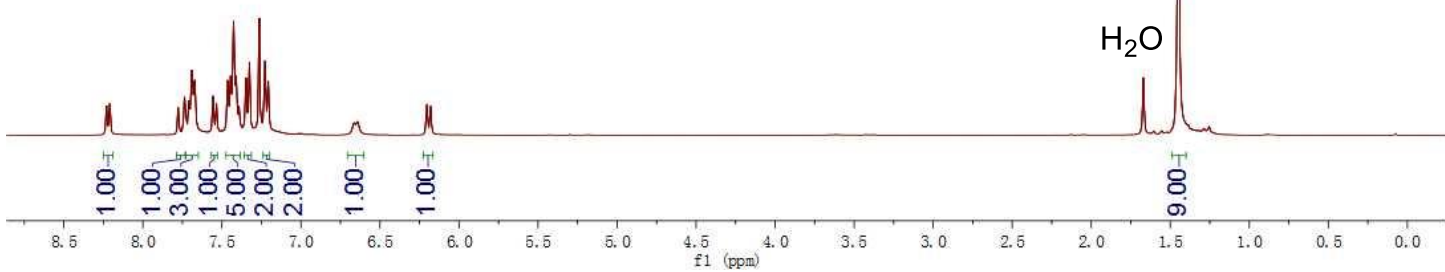

4l- ${ }^{13} \mathrm{C}\{1 \mathrm{H}\}$ NMR (150 $\left.\mathrm{MHz}, \mathrm{CDCl}_{3}\right)$

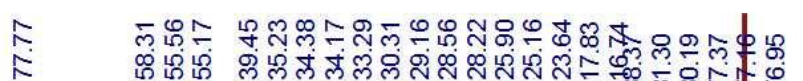

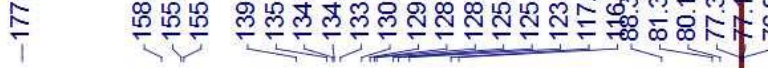

ํำ क़

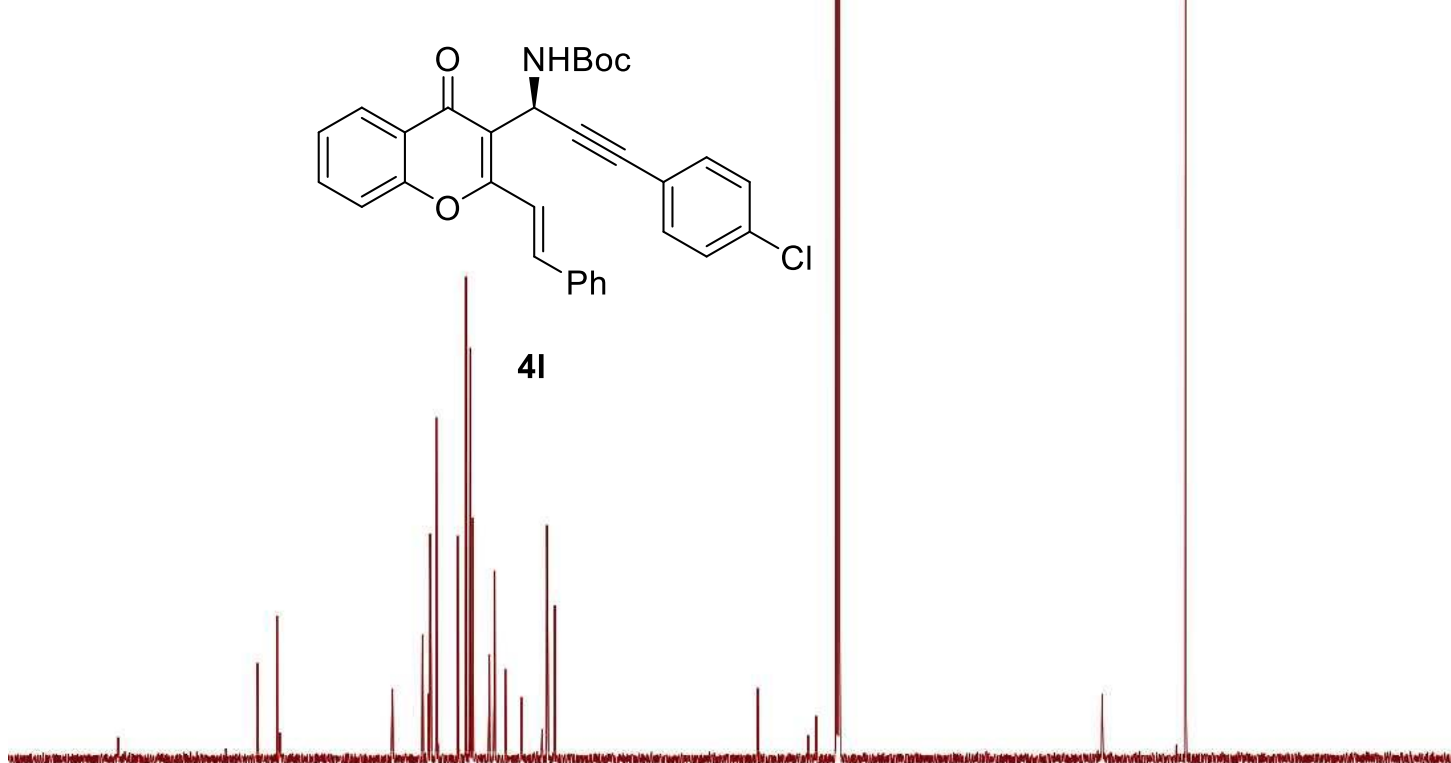

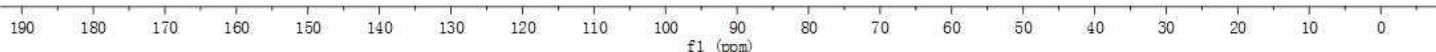




\section{4m- ${ }^{1} \mathrm{H}$ NMR (400 MHz, CDCl3)}

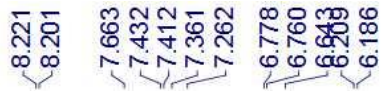

$\underset{\substack{1 \\ \stackrel{1}{8}}}{\stackrel{1}{2}}$

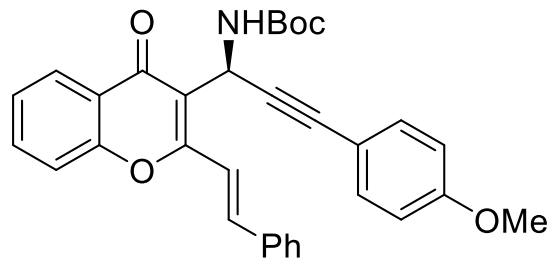

$4 m$

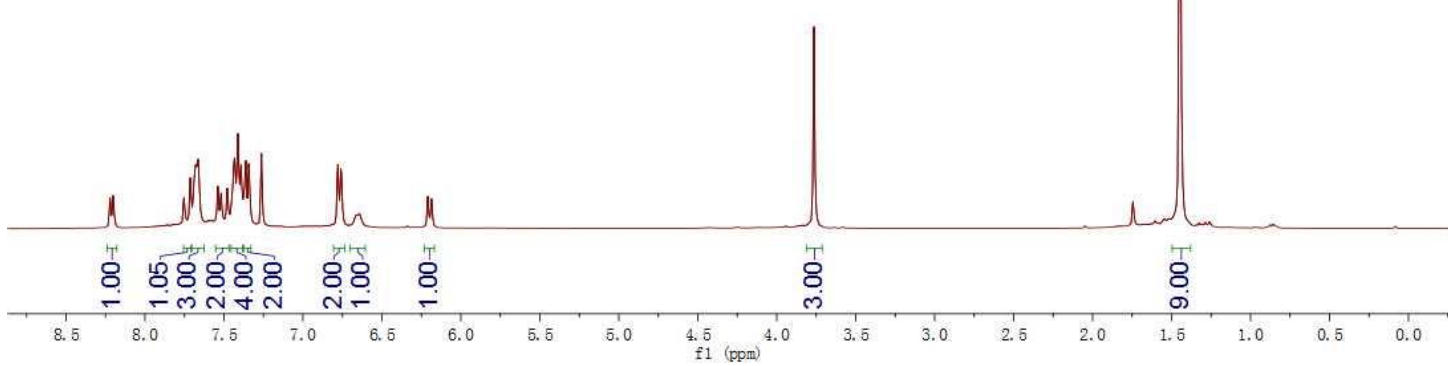

4m- ${ }^{13} \mathrm{C}\{1 \mathrm{H}\}$ NMR (150 MHz, $\left.\mathrm{CDCl}_{3}\right)$

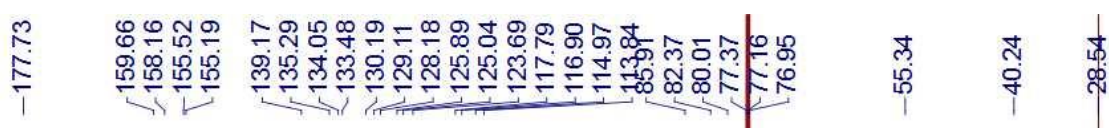<smiles>COc1ccc(C#C[C@H](N)c2c(/C=C/c3ccccc3)oc3ccccc3c2=O)cc1</smiles>
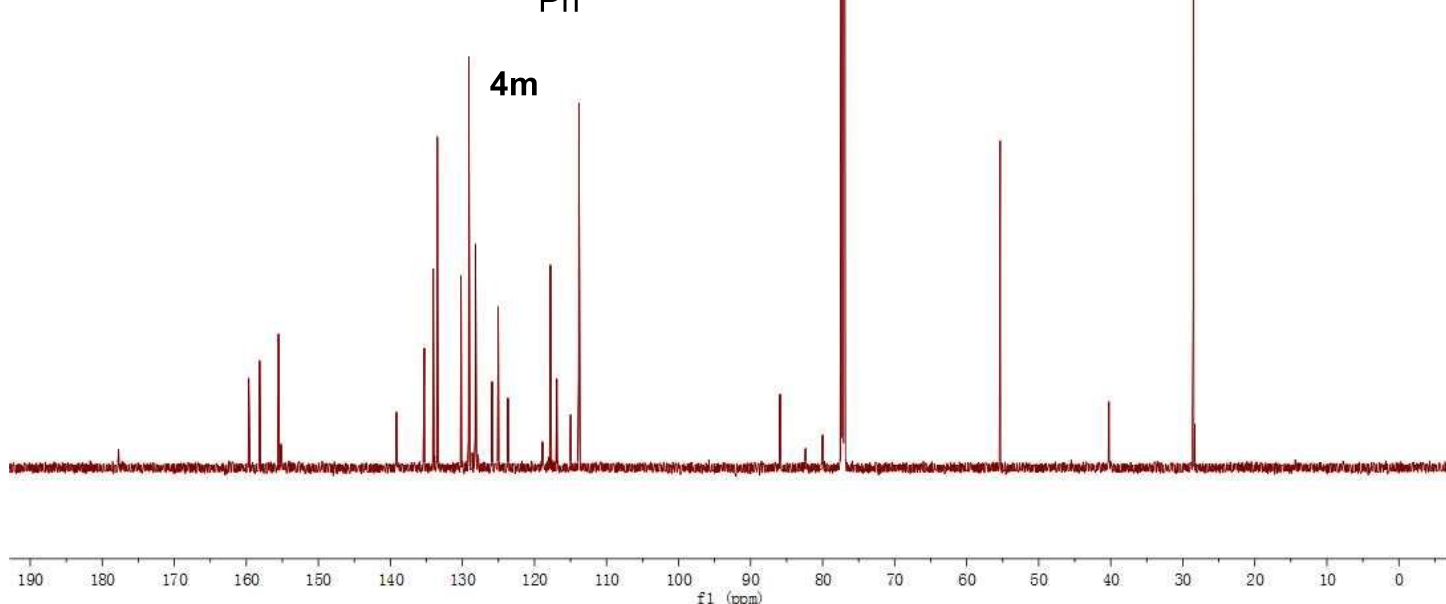
4n- ${ }^{1} \mathrm{H}$ NMR (400 $\left.\mathrm{MHz}, \mathrm{CDCl}_{3}\right)$

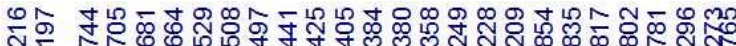

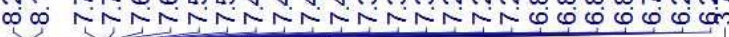<smiles>COC(=O)N[C@@H](C#Cc1ccccc1OC)c1c(/C=C/c2ccccc2)oc2ccccc2c1=O</smiles>

$4 n$

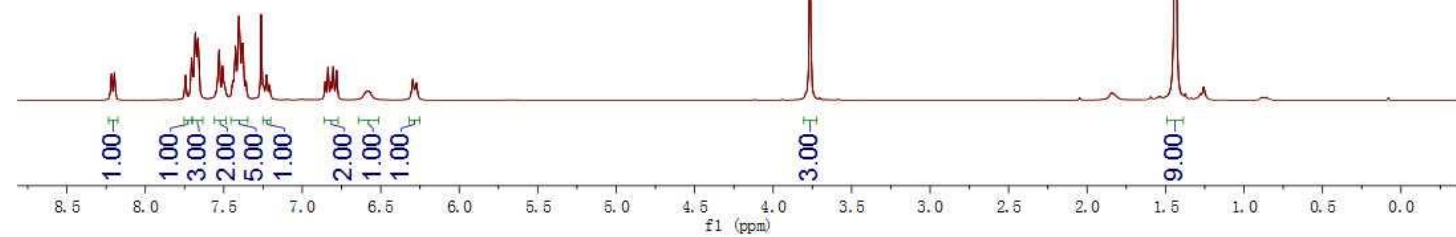

4n- ${ }^{13} \mathrm{C}\{1 \mathrm{H}\}$ NMR (150 MHz, CDCl 3$)$

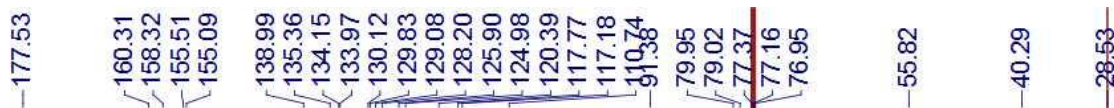

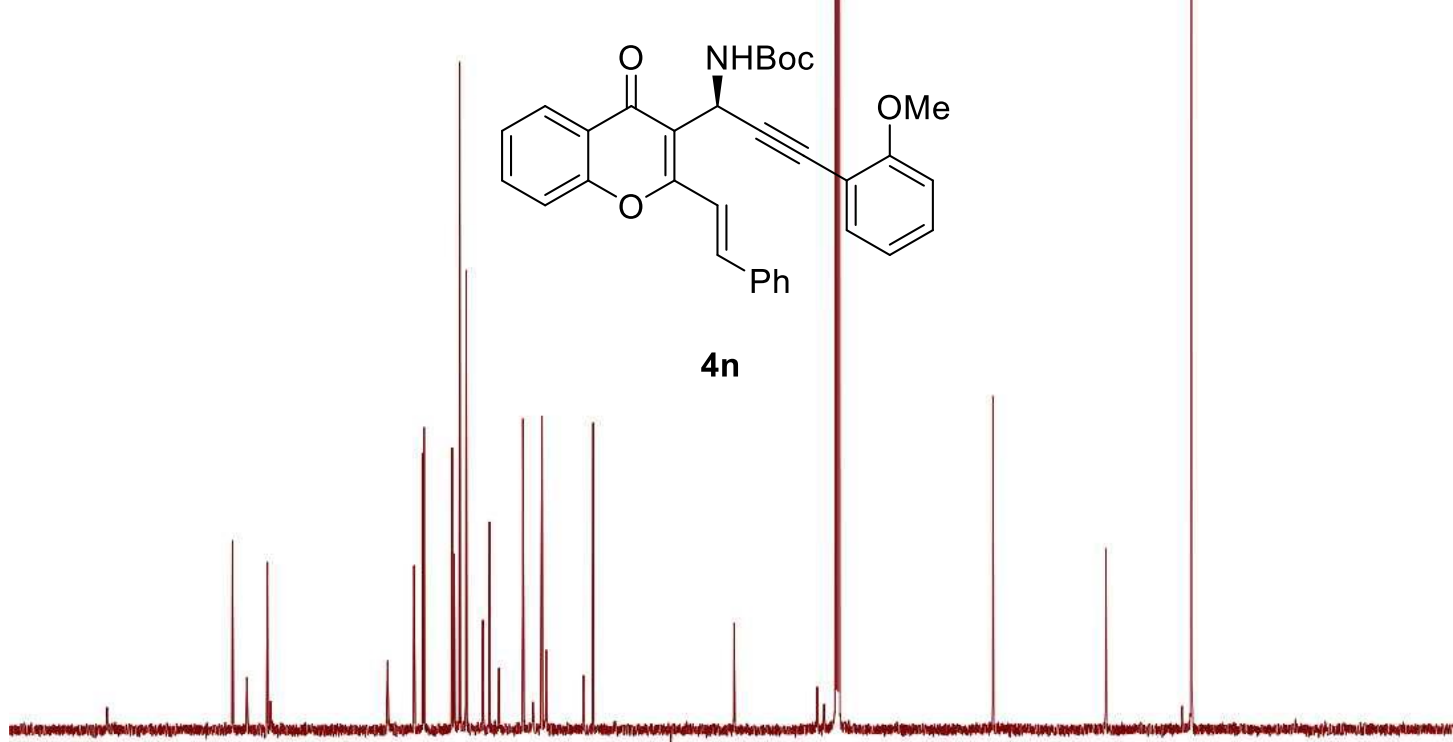

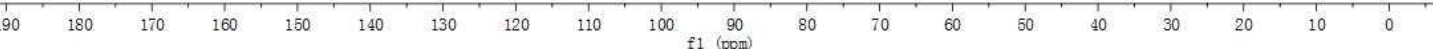




\section{0- ${ }^{1} \mathrm{H}$ NMR (400 MHz, CDCl3)}

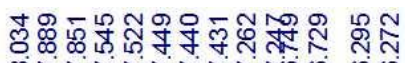

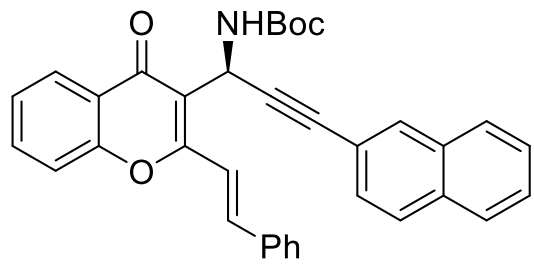

40

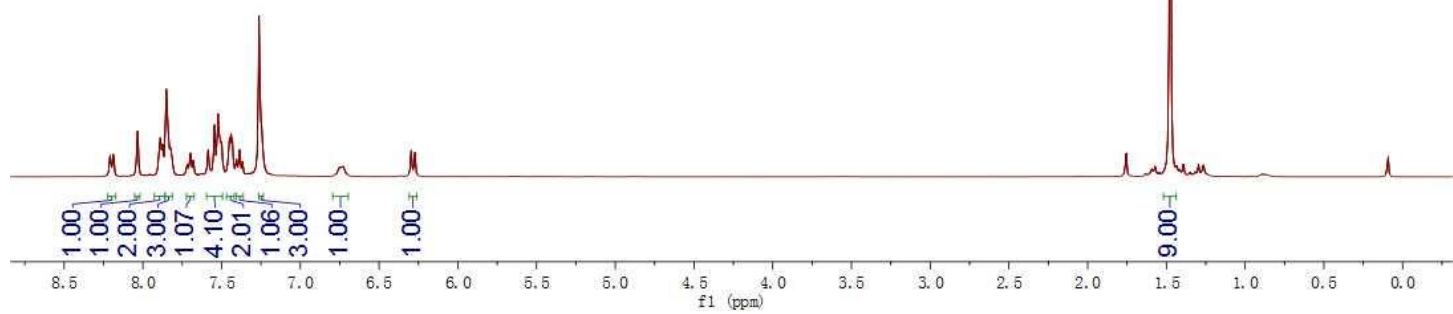

40- ${ }^{13} \mathrm{C}\{1 \mathrm{H}\}$ NMR (150 $\left.\mathrm{MHz}, \mathrm{CDCl}_{3}\right)$

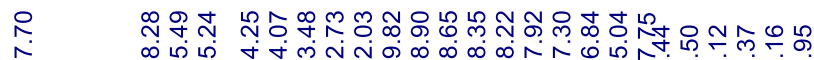

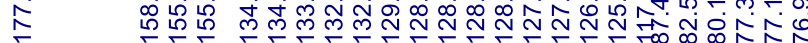

更

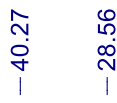<smiles>CC(C)(C)OC(=O)N[C@@H](C#Cc1ccc2ccccc2c1)c1c(/C=C/c2ccccc2)oc2ccccc2c1=O</smiles>

40

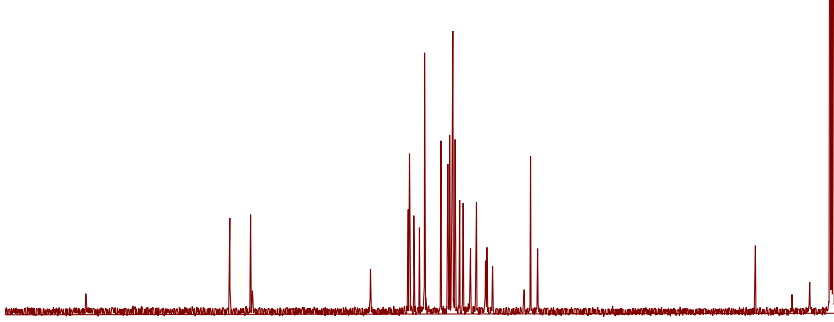

$\begin{array}{llllllllll}180 & 170 & 160 & 150 & 140 & 130 & 120 & 110 & 100 & 90 \\ \mathrm{f1} & (\mathrm{ppm})\end{array}$ 
4p- ${ }^{1} \mathrm{H}$ NMR (400 MHz, $\left.\mathrm{CDCl}_{3}\right)$

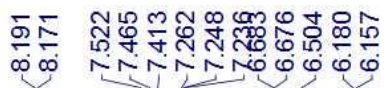

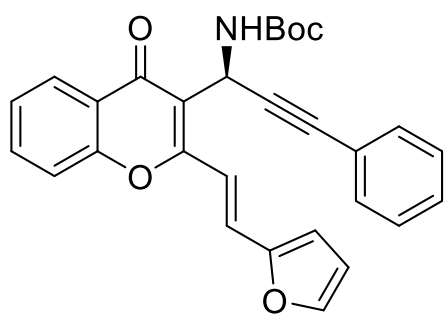

$4 p$

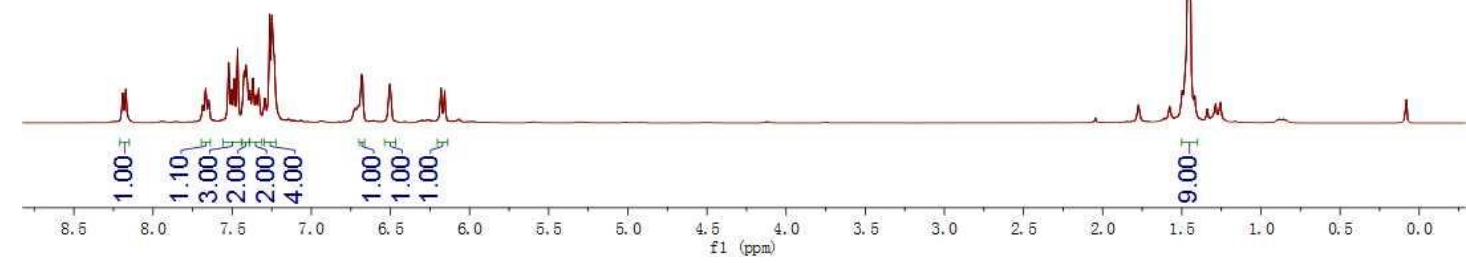

4p- ${ }^{13} \mathrm{C}\{1 \mathrm{H}\}$ NMR (150 MHz, $\left.\mathrm{CDCl}_{3}\right)$

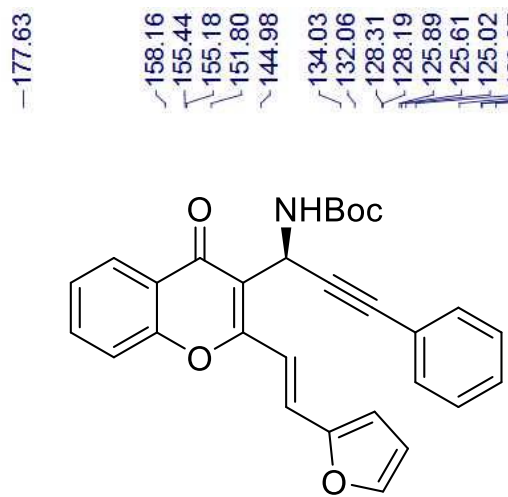

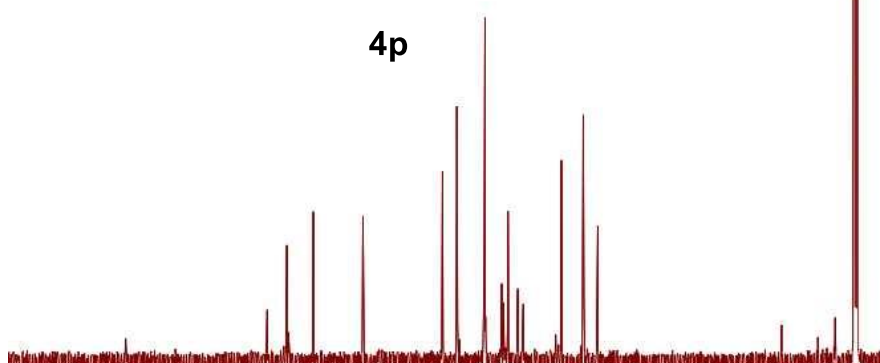

ঙัy

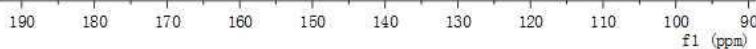


4q- ${ }^{1} \mathrm{H}$ NMR (400 $\left.\mathrm{MHz}, \mathrm{CDCl}_{3}\right)$

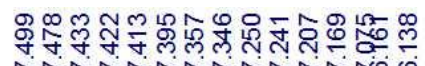

NNNNNNNNNNAONO<smiles>CC(C)(C)OC(=O)C(C#Cc1ccccc1)c1c(/C=C/c2cccs2)oc2ccccc2c1=O</smiles>

$4 q$

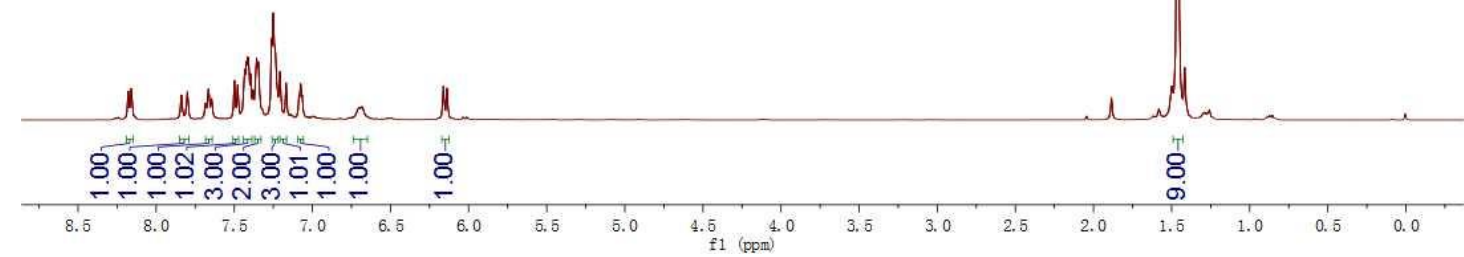

4q- ${ }^{13} \mathrm{C}\{1 \mathrm{H}\}$ NMR (150 MHz, $\left.\mathrm{CDCl}_{3}\right)$

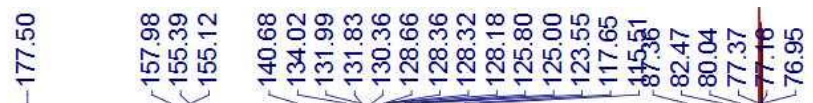

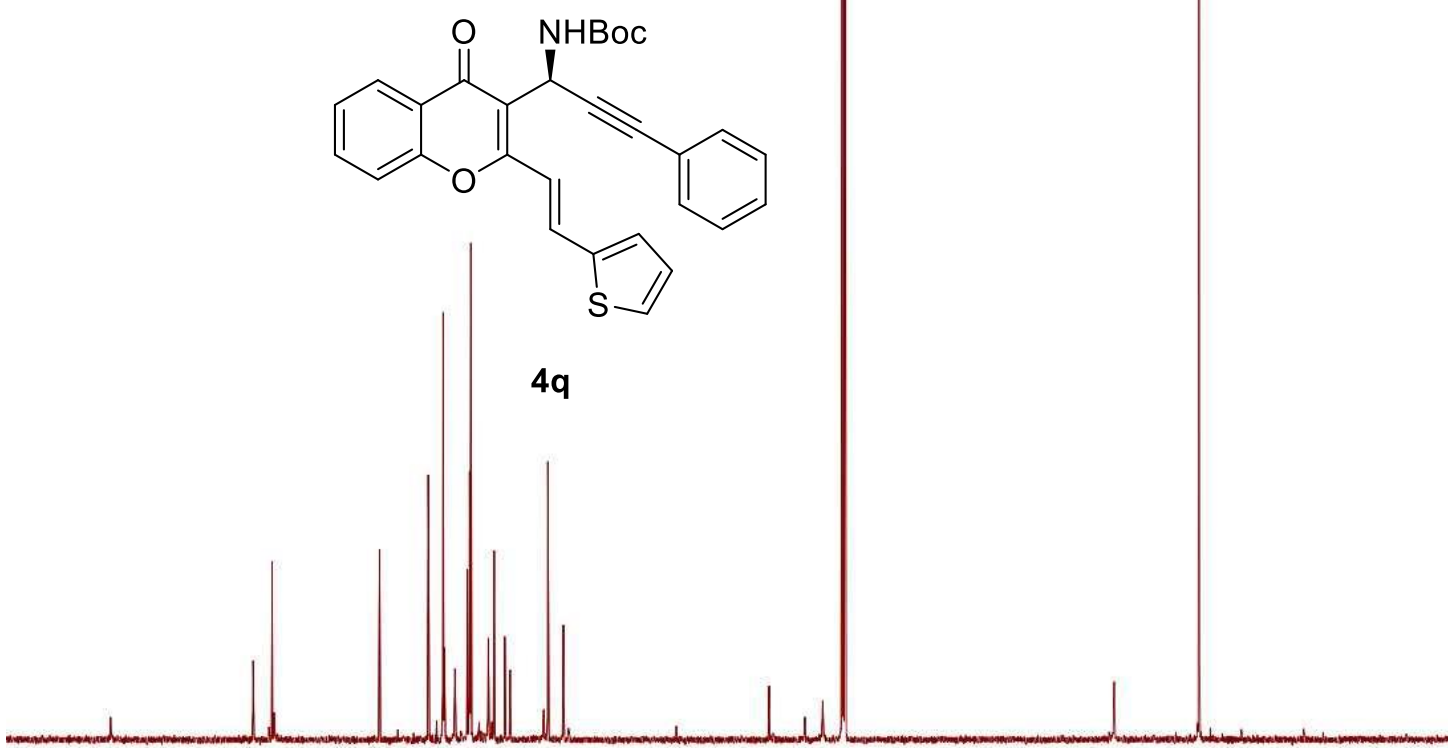

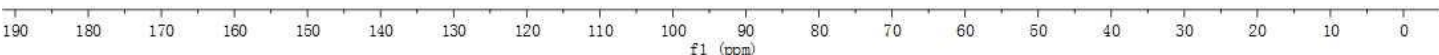


4r- ${ }^{1} \mathrm{H}$ NMR (400 MHz, CDCl3)

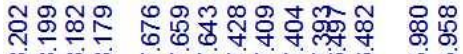

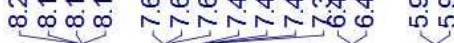

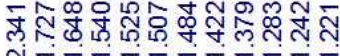<smiles>CC(C)(C)OC(=O)N[C@@H](C#CC1CCCCC1)c1c(/C=C/c2ccccc2)oc2ccccc2c1=O</smiles>

$4 r$

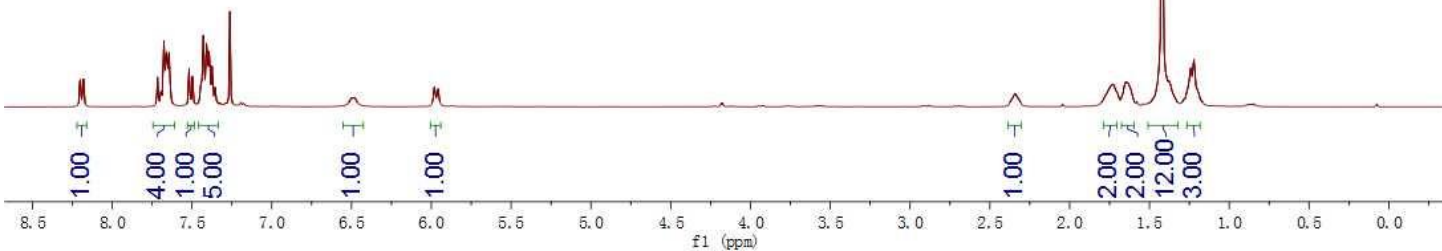

4r- ${ }^{13} \mathrm{C}\{1 \mathrm{H}\} \mathrm{NMR}\left(150 \mathrm{MHz}, \mathrm{CDCl}_{3}\right)$

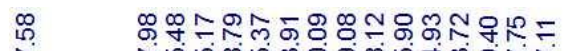

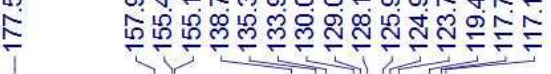

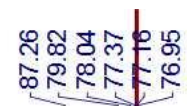

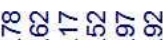

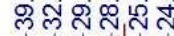
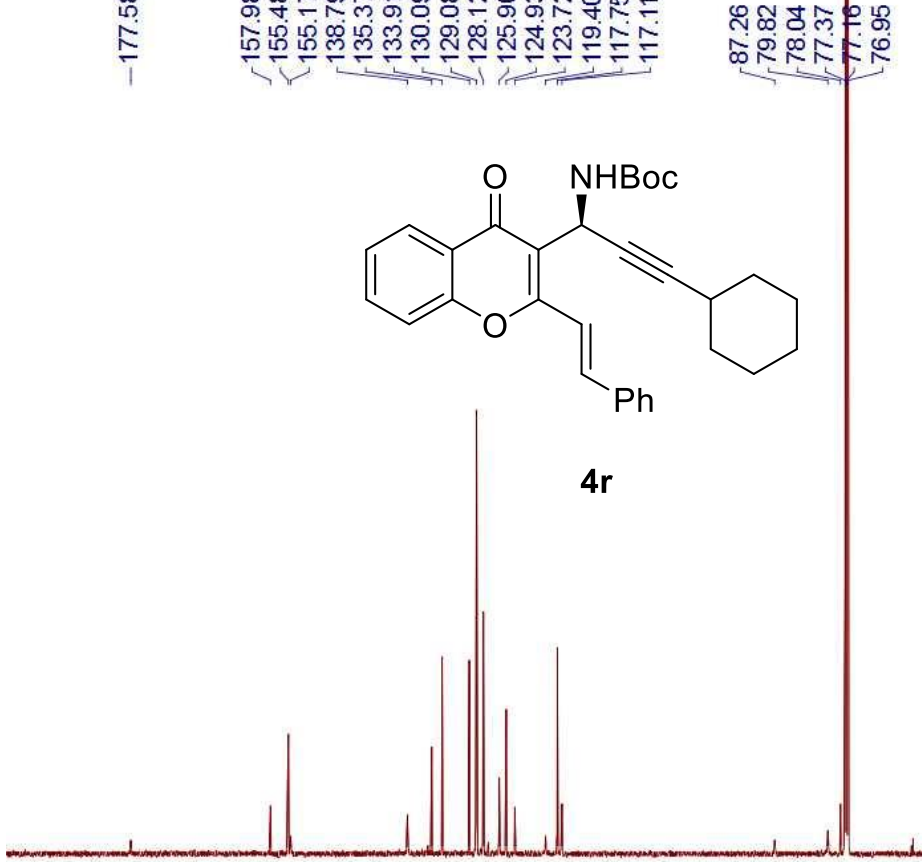

$\begin{array}{llllllllll}190 & 180 & 170 & 160 & 150 & 140 & 130 & 120 & 110 & 100\end{array}$ 


\section{6a- ${ }^{1} \mathrm{H}$ NMR (400 $\left.\mathrm{MHz}, \mathrm{CDCl}_{3}\right)$}

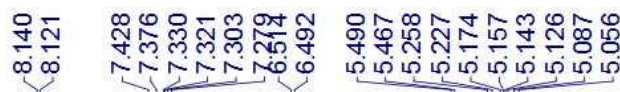<smiles>Cc1oc2ccccc2c(=O)c1[C@H](NC(=O)OCc1ccccc1)C(=O)OCc1ccccc1</smiles>

$6 a$

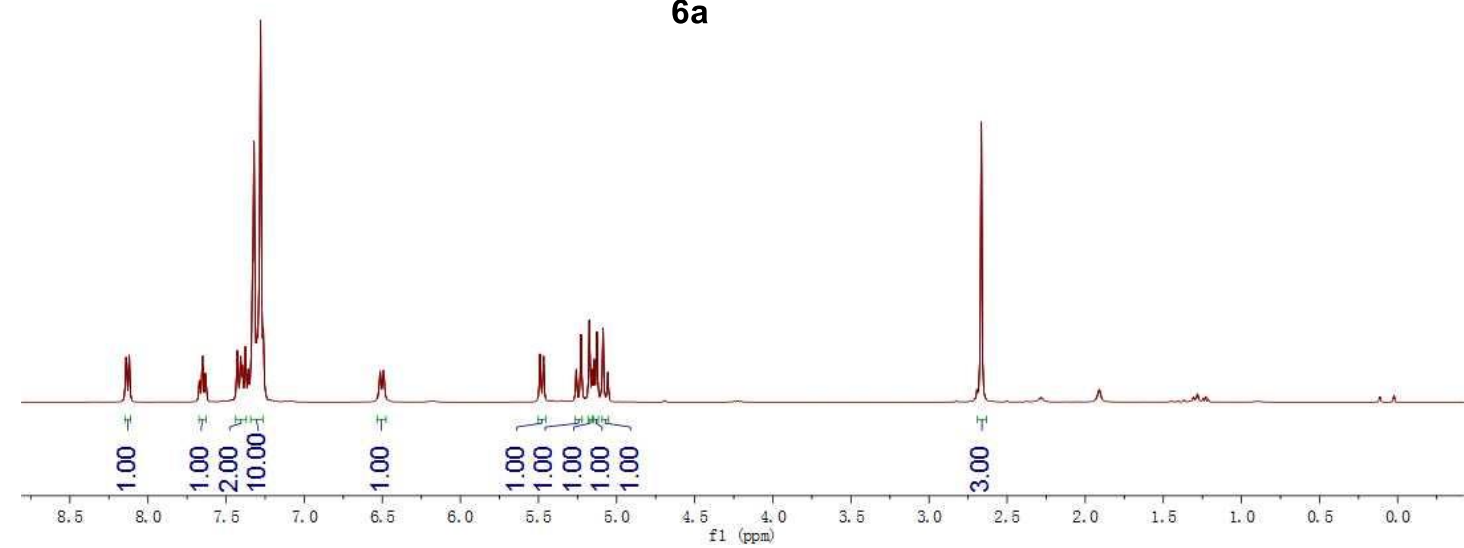

6a- ${ }^{13} \mathrm{C}\{1 \mathrm{H}\}$ NMR (100 MHz, $\left.\mathrm{CDCl}_{3}\right)$

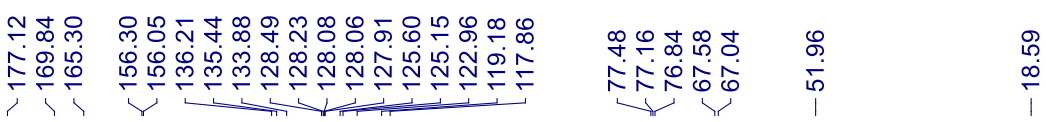

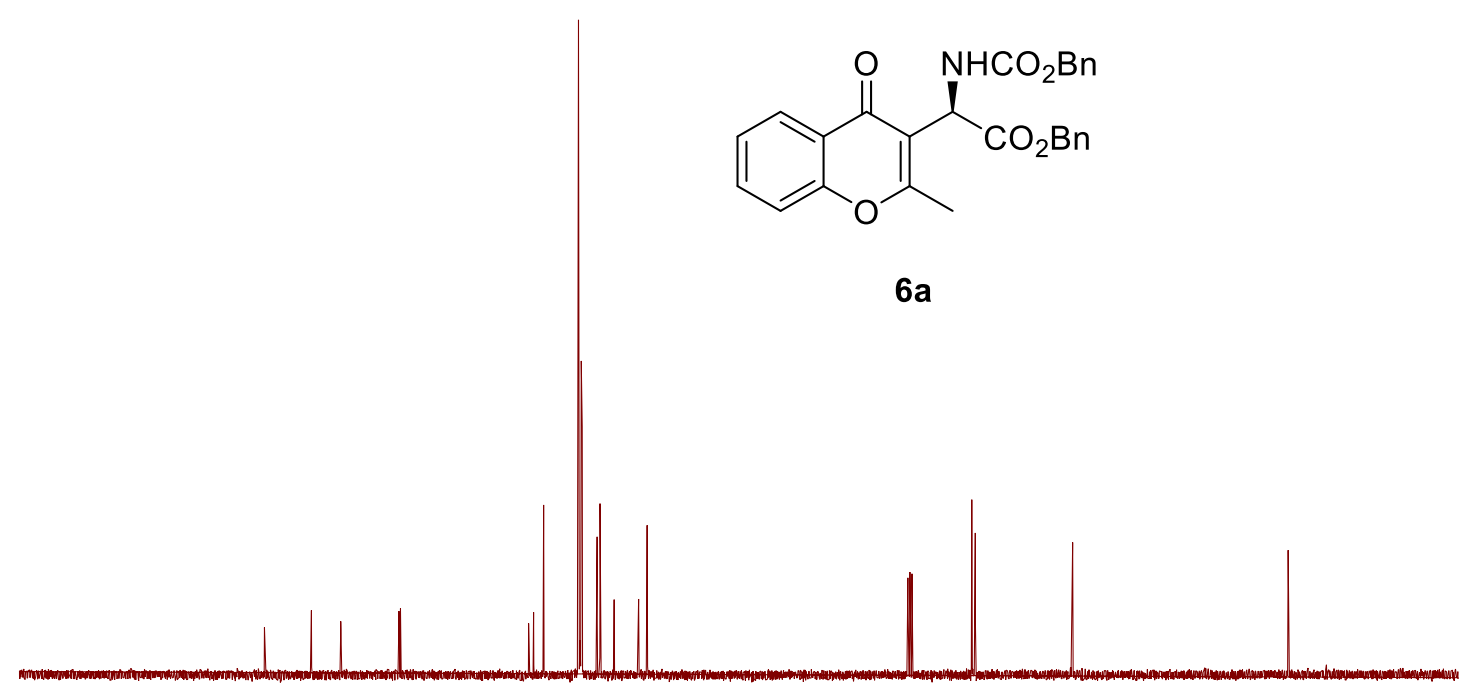

$\begin{array}{llllllllllll}210 & 200 & 190 & 180 & 170 & 160 & 150 & 140 & 130 & 120 & 110 & 100\end{array}$ 
6b- ${ }^{1} \mathrm{H}$ NMR (400 MHz, CDCl3)

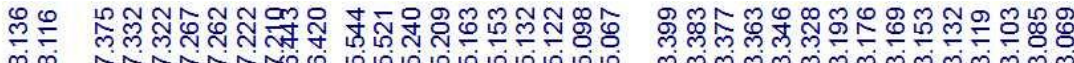

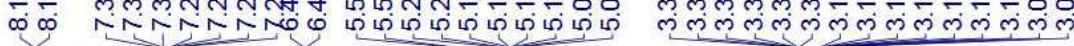

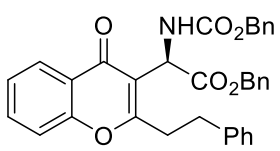

$6 b$

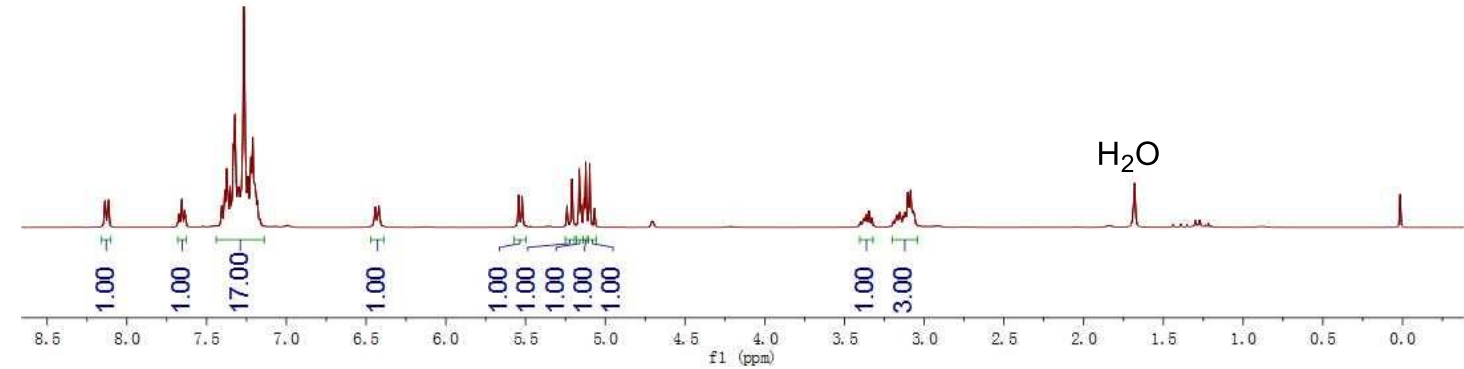

6b- ${ }^{13} \mathrm{C}\{1 \mathrm{H}\} \mathrm{NMR}\left(100 \mathrm{MHz}, \mathrm{CDCl}_{3}\right)$

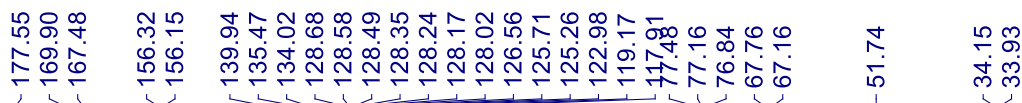<smiles>O=C(N[C@H](Cc1ccccc1)c1c(CCc2ccccc2)oc2ccccc2c1=O)c1ccccc1</smiles>

$6 b$

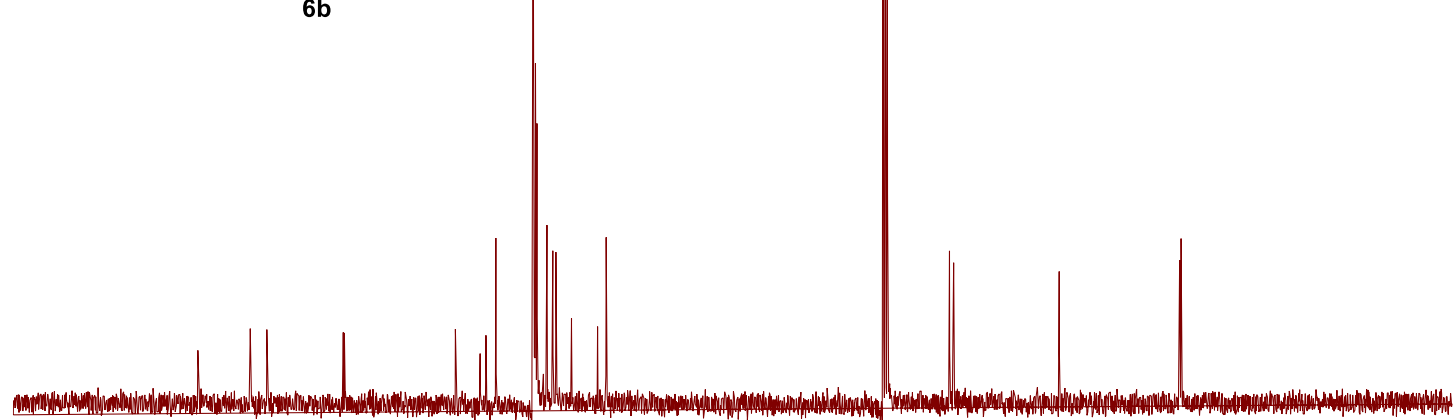

$200 \quad 190 \quad 18$

$160 \quad 150$

$40 \quad 130$

$\stackrel{100}{\mathrm{f} 1}(\mathrm{ppm})$ 


\section{6c- ${ }^{1} \mathrm{H}$ NMR (400 MHz, $\left.\mathrm{CDCl}_{3}\right)$}

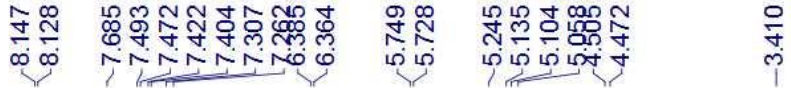

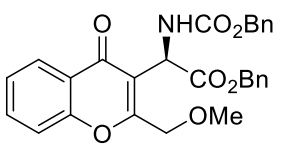

$6 c$

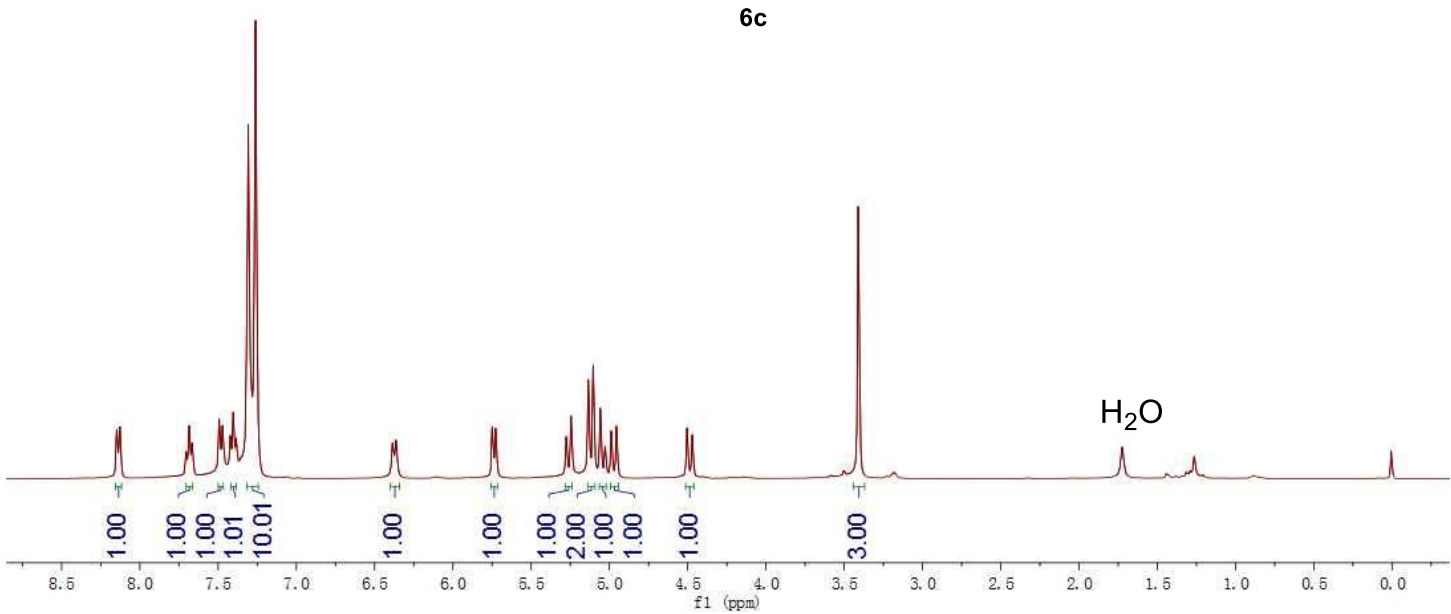

6c- ${ }^{13} \mathrm{C}\{1 \mathrm{H}\}$ NMR $\left(100 \mathrm{MHz}, \mathrm{CDCl}_{3}\right)$

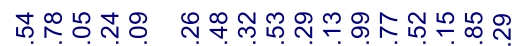

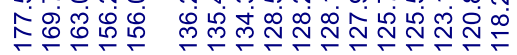

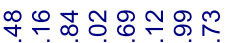

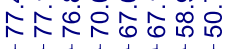

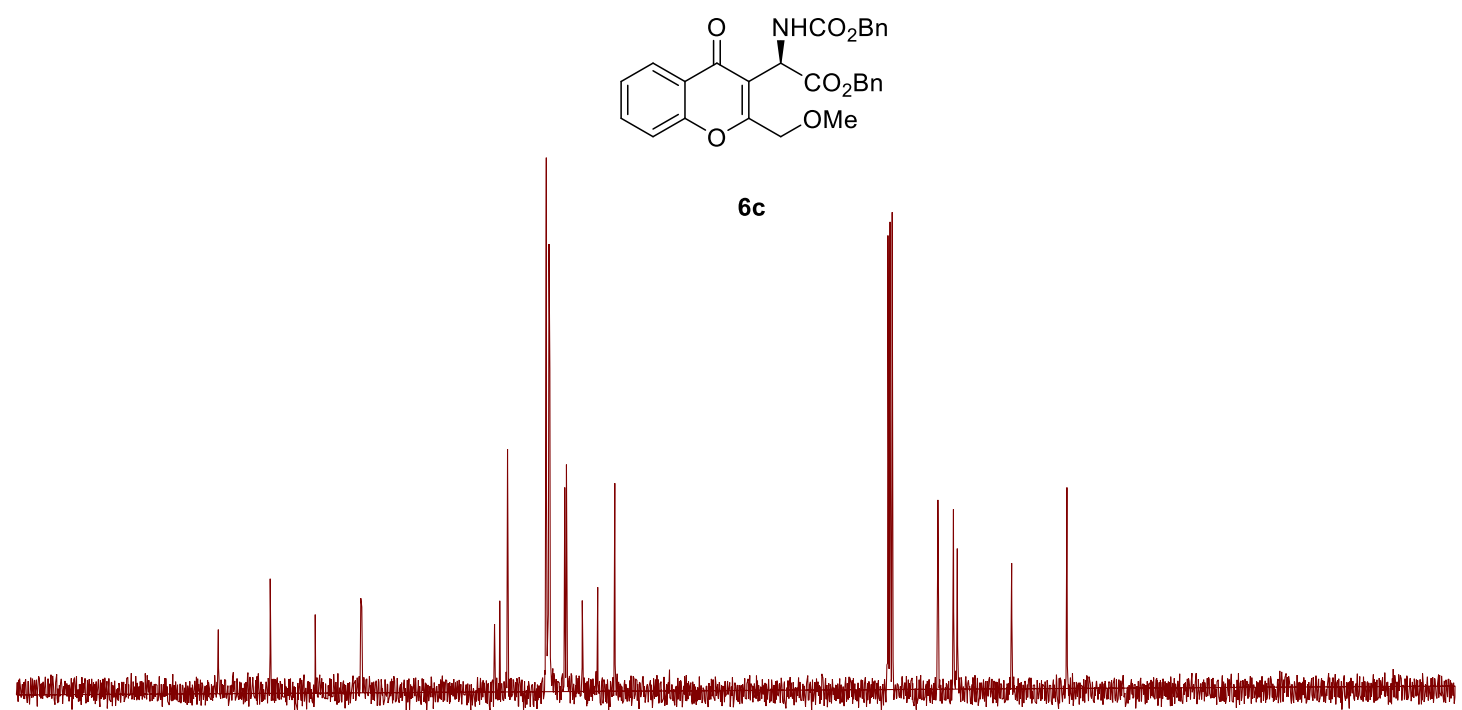

$6 c$

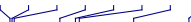

$\begin{array}{llll}200 & 190 & 180 & 170\end{array}$

150

130

$110 \quad 100$ 
6d- ${ }^{1} \mathrm{H}$ NMR (400 MHz, $\left.\mathrm{CDCl}_{3}\right)$

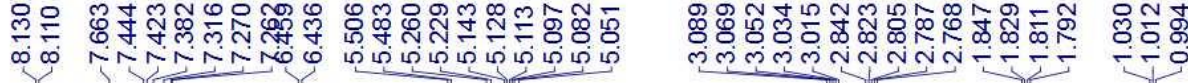

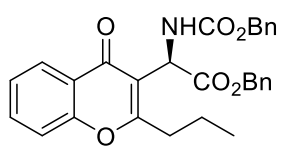

6d

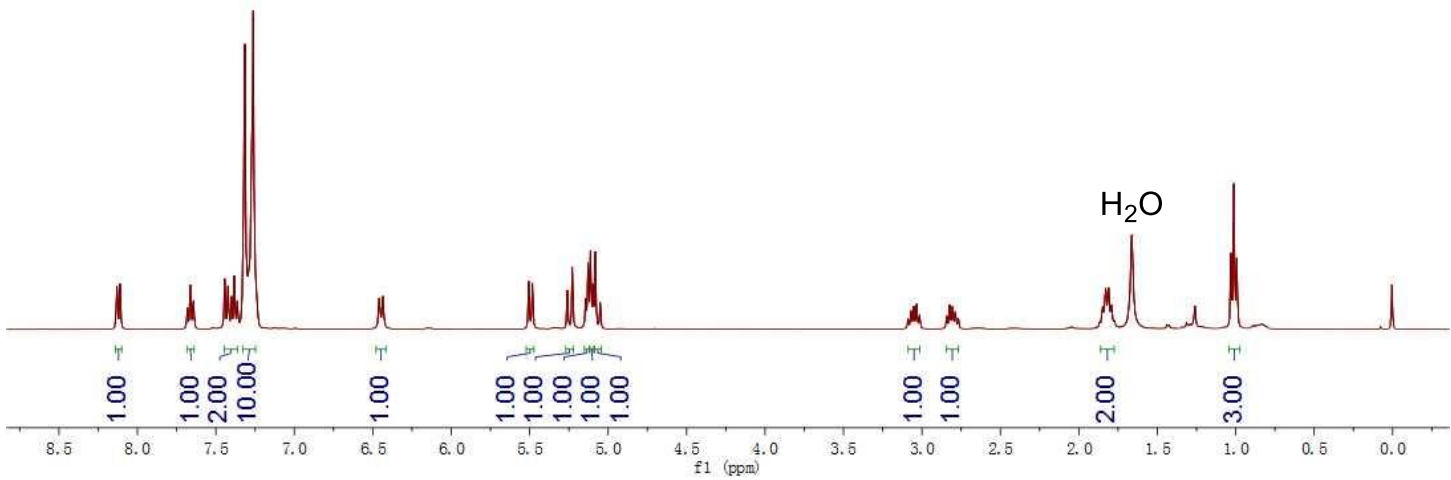

6d- ${ }^{13} \mathrm{C}\{1 \mathrm{H}\}$ NMR (100 $\left.\mathrm{MHz}, \mathrm{CDCl}_{3}\right)$

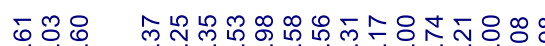

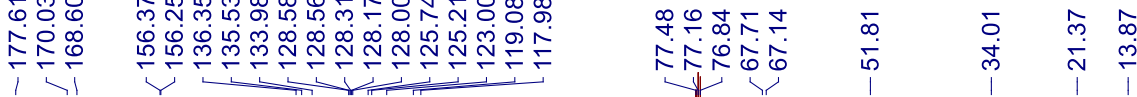

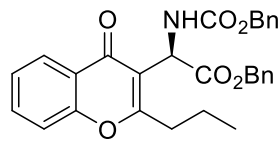

$6 d$

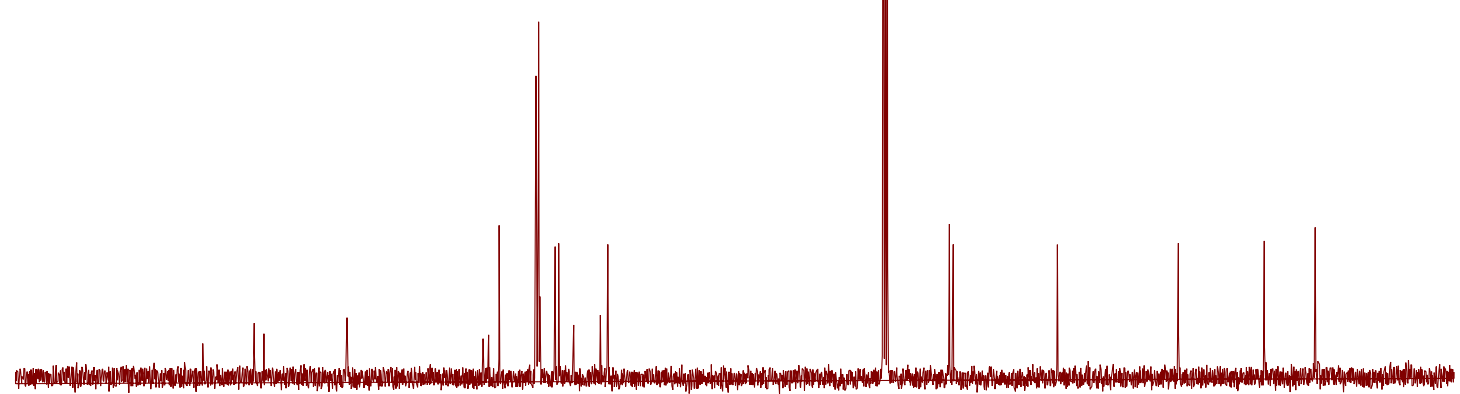

$\begin{array}{lllllllllll}200 & 190 & 180 & 170 & 160 & 150 & 140 & 130 & 120 & 110 & 100\end{array}$ 


\section{6e- ${ }^{1} \mathrm{H}$ NMR (400 MHz, CDCl3) \\ 牙 궁

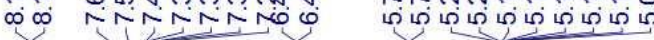

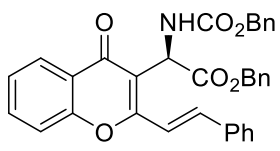

6

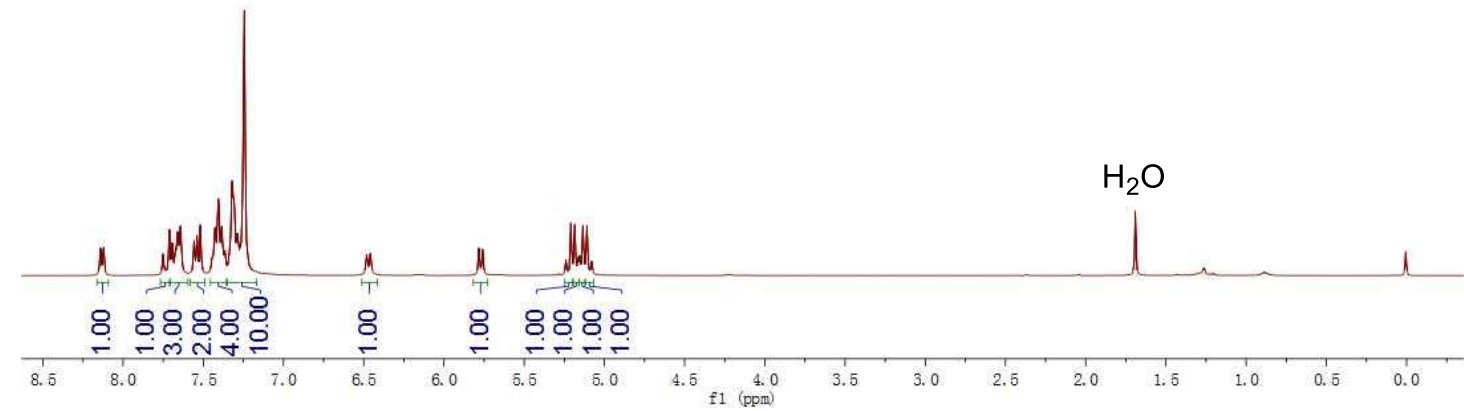

6e- ${ }^{13} \mathrm{C}\{1 \mathrm{H}\}$ NMR (100 MHz, $\left.\mathrm{CDCl}_{3}\right)$

๓

它

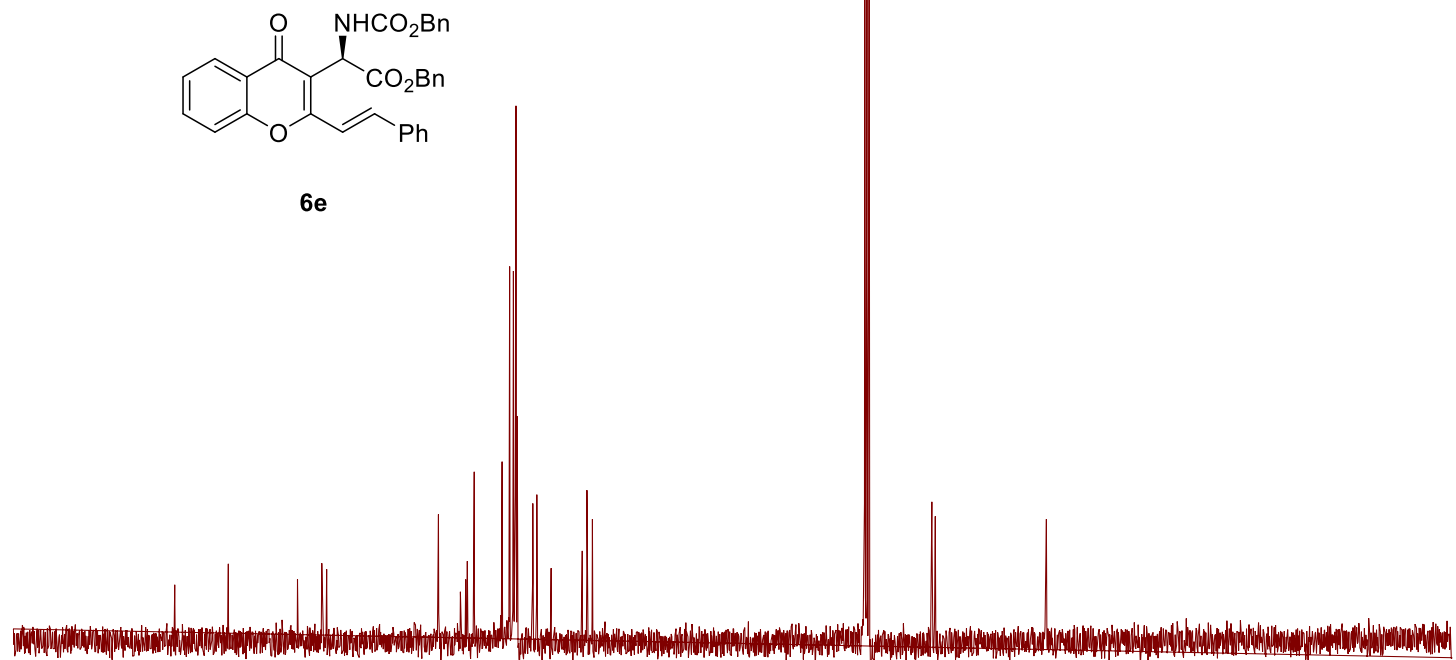

$\begin{array}{lllllllllll}190 & 180 & 170 & 160 & 150 & 140 & 130 & 120 & 110 & 100 & 90\end{array}$ 
6f- ${ }^{1} \mathrm{H}$ NMR (400 MHz, $\left.\mathrm{CDCl}_{3}\right)$

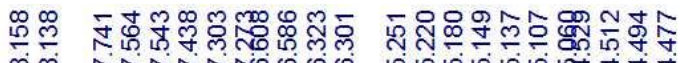

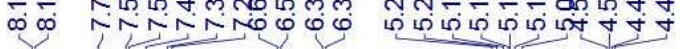
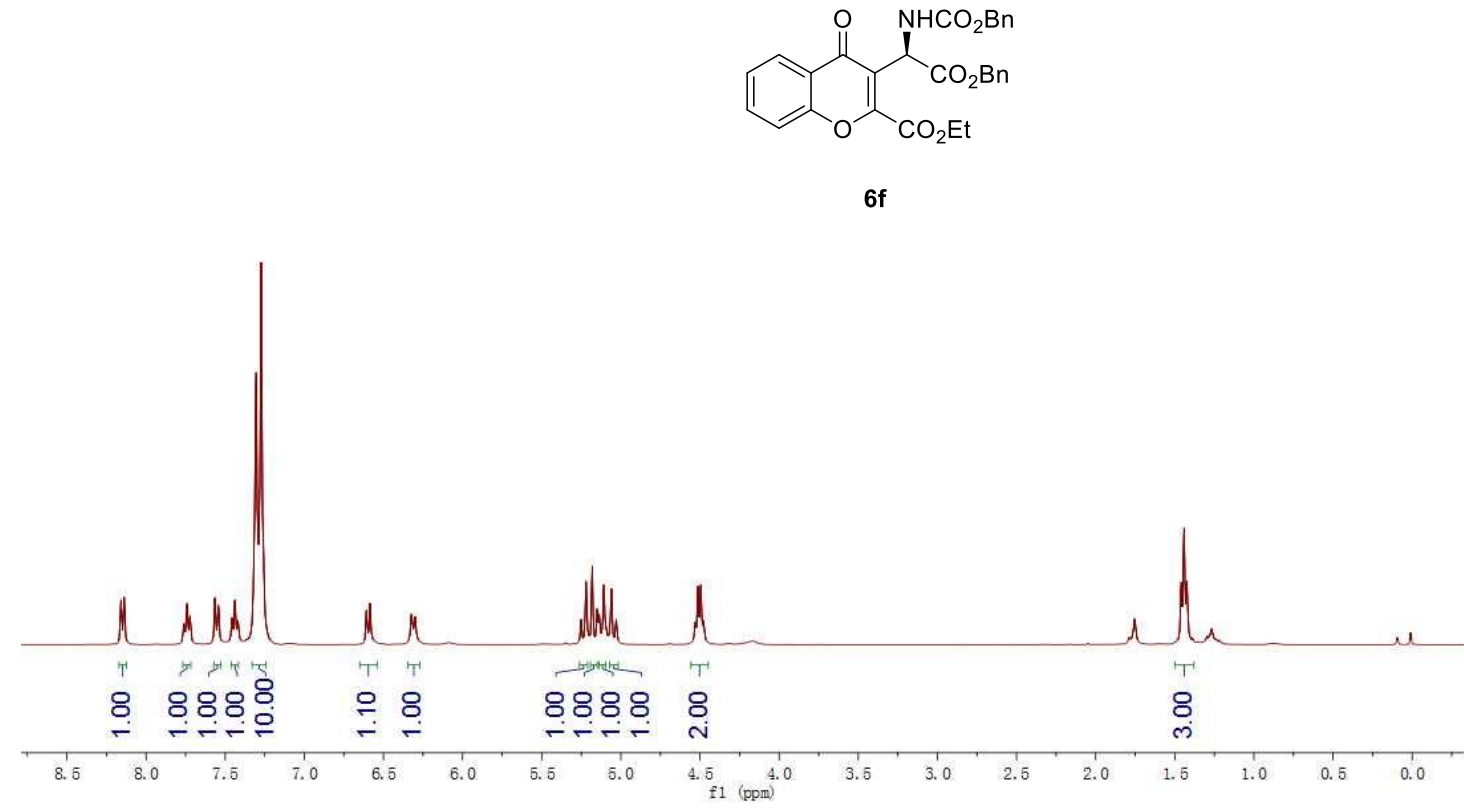

6f- ${ }^{13} \mathrm{C}\{1 \mathrm{H}\}$ NMR (100 $\left.\mathrm{MHz}, \mathrm{CDCl}_{3}\right)$

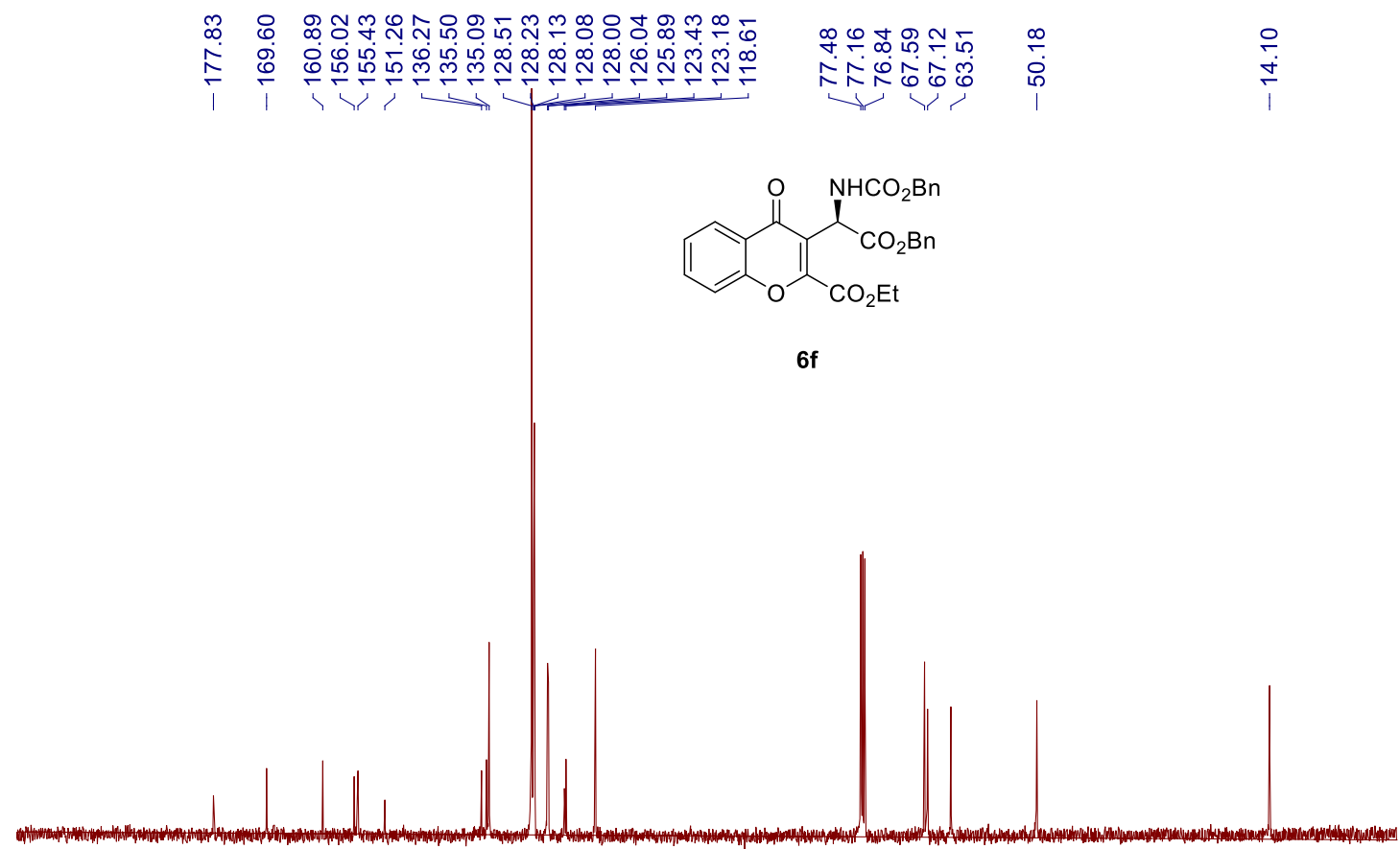

$200 \quad 190$

$180 \quad 170$

$60 \quad 150$

$40 \quad 130$

$10 \stackrel{100}{\mathrm{f} 1}(\mathrm{ppm})$ 


\section{6g- ${ }^{1} \mathrm{H}$ NMR (400 MHz, CDCl 3$)$}

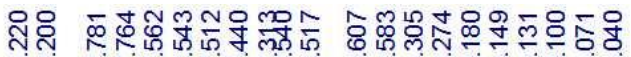

$\infty \infty$ T.

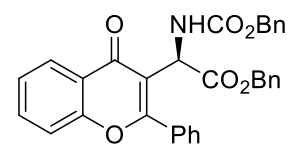

$6 g$

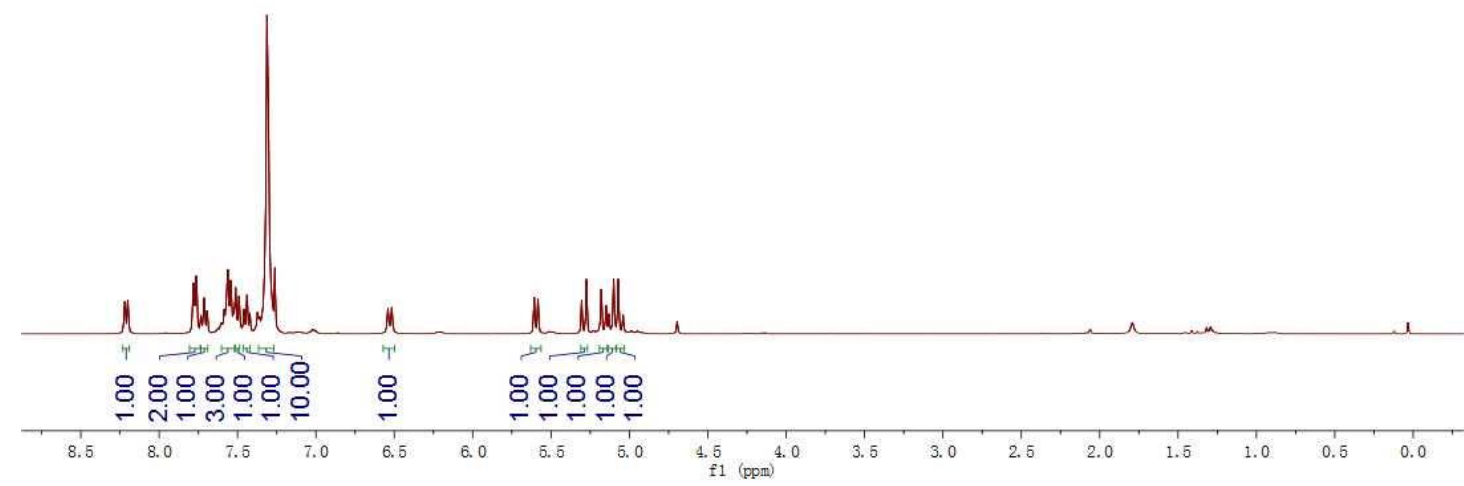

6g- ${ }^{13} \mathrm{C}\{1 \mathrm{H}\} \mathrm{NMR}\left(100 \mathrm{MHz}, \mathrm{CDCl}_{3}\right)$

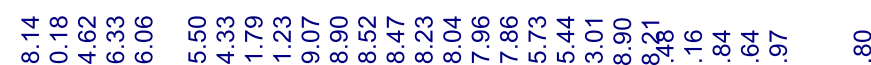

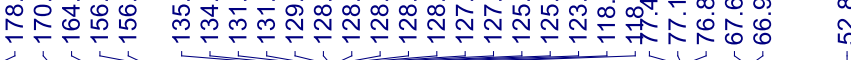

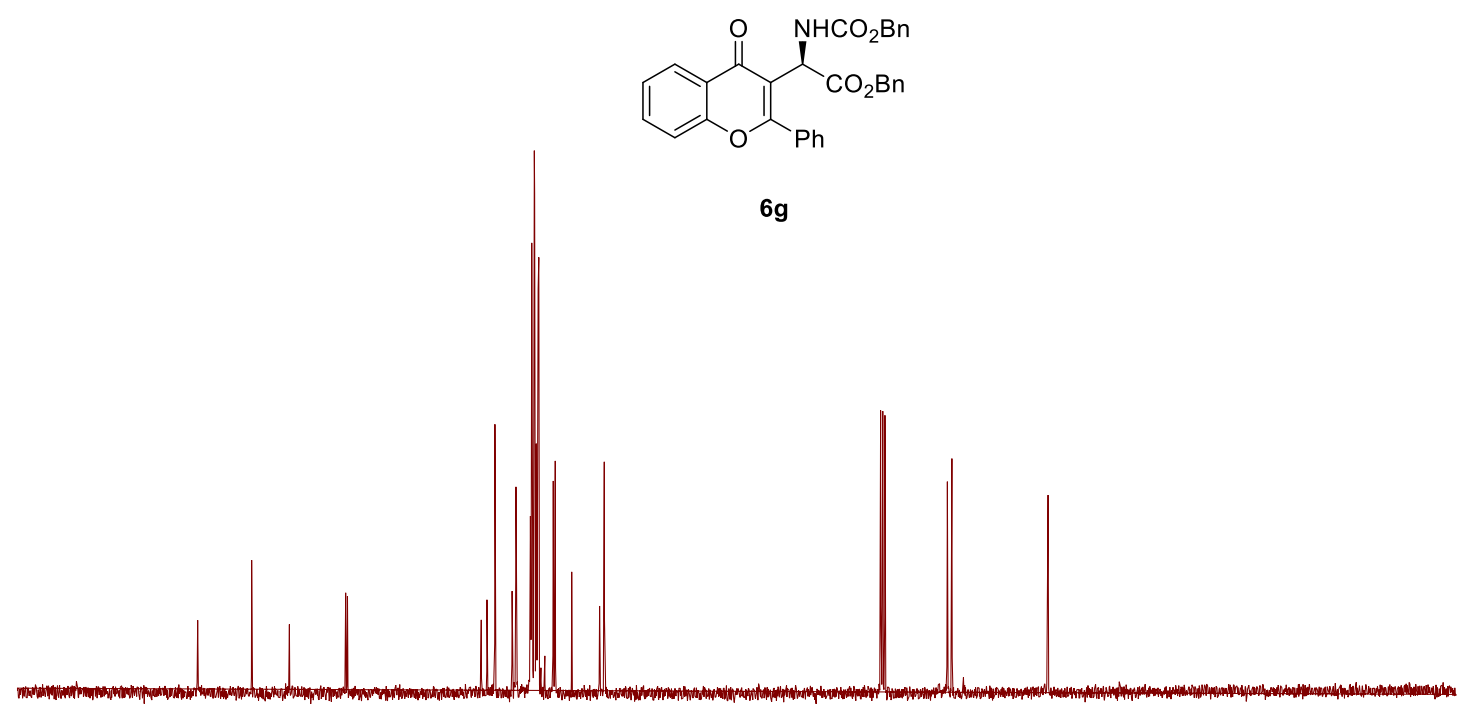

$\begin{array}{lllll}200 & 190 & 180 & 170 & 160\end{array}$ $\stackrel{100}{\mathrm{f} 1}(\mathrm{ppm})$ 
6h- ${ }^{1} \mathrm{H}$ NMR (400 MHz, CDCl3)

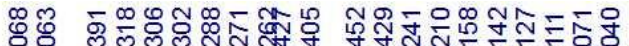

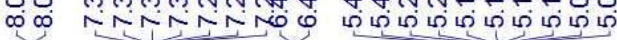<smiles>Cc1oc2ccc(Cl)cc2c(=O)c1C(NC(=O)OCc1ccccc1)C(=O)O</smiles>

$6 \mathrm{~h}$

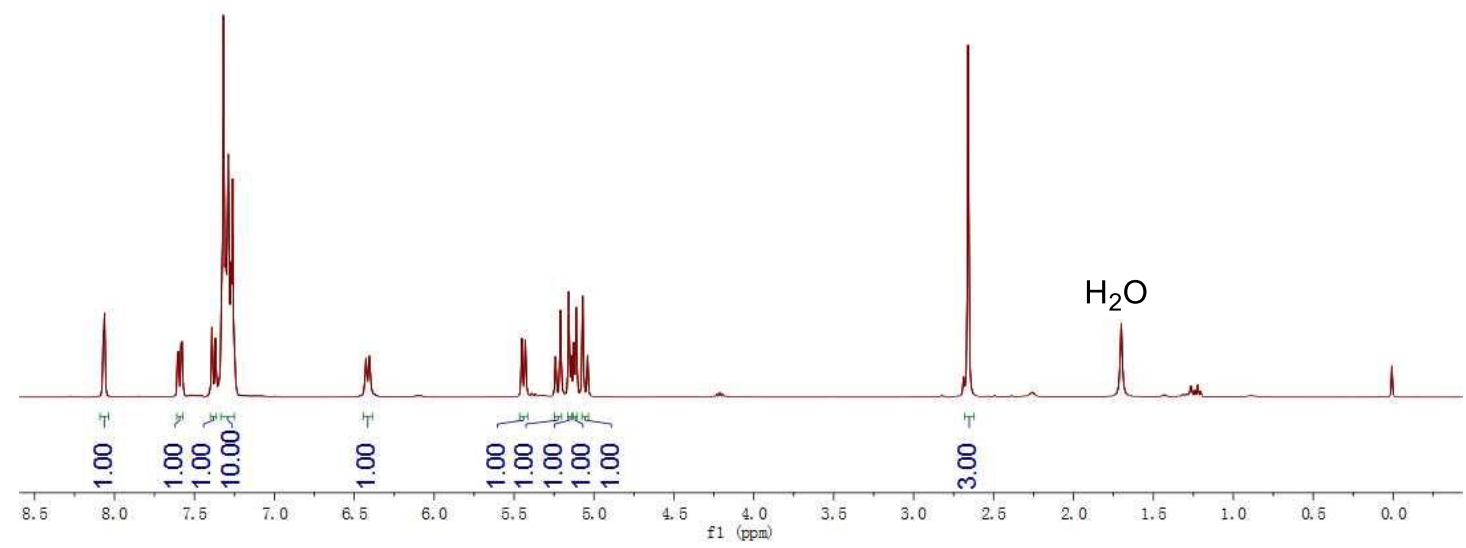

6h- ${ }^{13} \mathrm{C}\{1 \mathrm{H}\}$ NMR (100 MHz, $\left.\mathrm{CDCl}_{3}\right)$

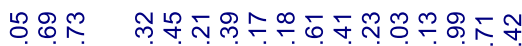

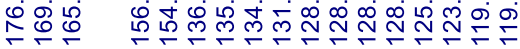

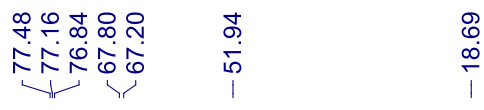

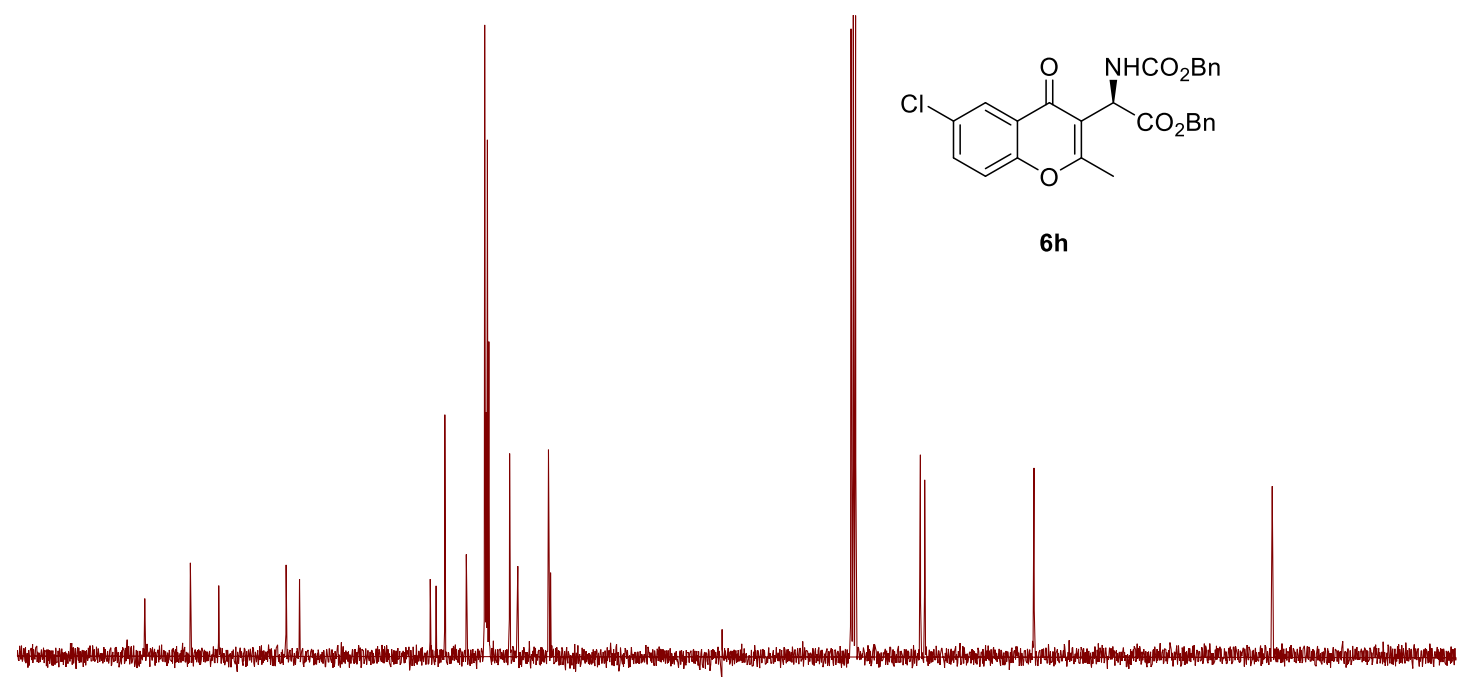

$\begin{array}{lllllllllll}190 & 180 & 170 & 160 & 150 & 140 & 130 & 120 & 110 & 100 & 90\end{array}$ 


\section{6i- ${ }^{1} \mathrm{H}$ NMR (400 $\left.\mathrm{MHz}, \mathrm{CDCl}_{3}\right)$}

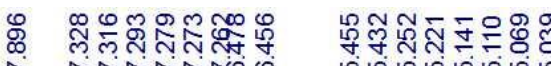

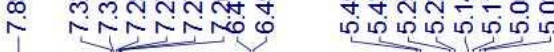

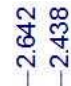

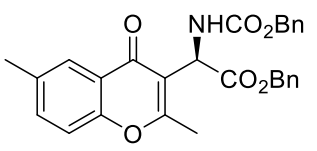

$6 \mathbf{i}$

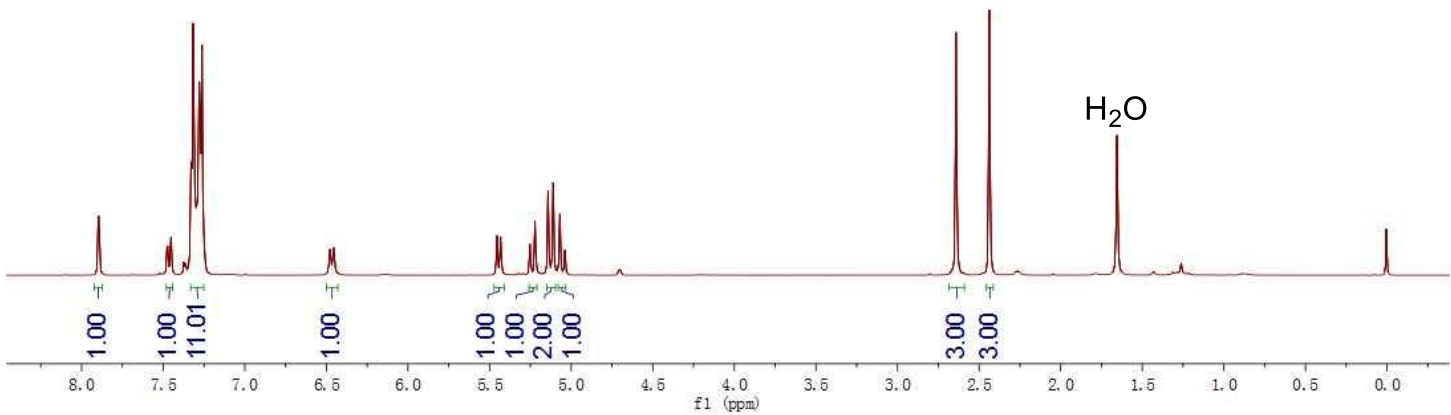

6i- ${ }^{13} \mathrm{C}\{1 \mathrm{H}\}$ NMR (100 MHz, $\left.\mathrm{CDCl}_{3}\right)$

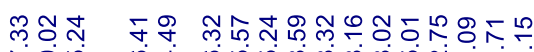

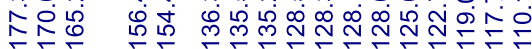

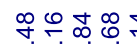

Кㅅำ

ชิ

도옹

人)<smiles>Cc1ccc2oc(C)c(C(NC(=O)OCc3ccccc3)C(=O)OCc3ccccc3)c(=O)c2c1</smiles>

$6 \mathbf{i}$

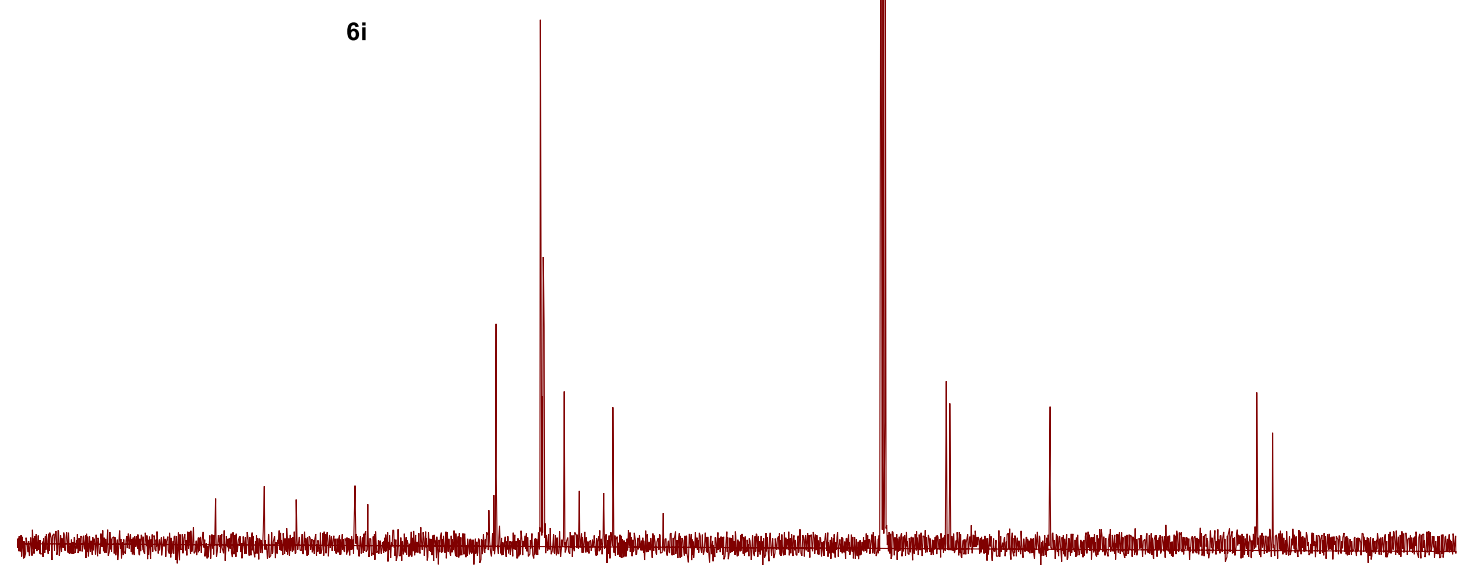

$\begin{array}{lllll}200 & 190 & 180 & 170 & 160\end{array}$

150

$40 \quad 130$

$20 \quad 110$

100 
6j- ${ }^{1} \mathrm{H}$ NMR (400 MHz, $\left.\mathrm{CDCl}_{3}\right)$

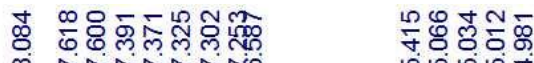

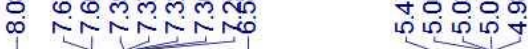

品

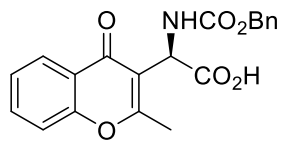

$6 \mathbf{j}$

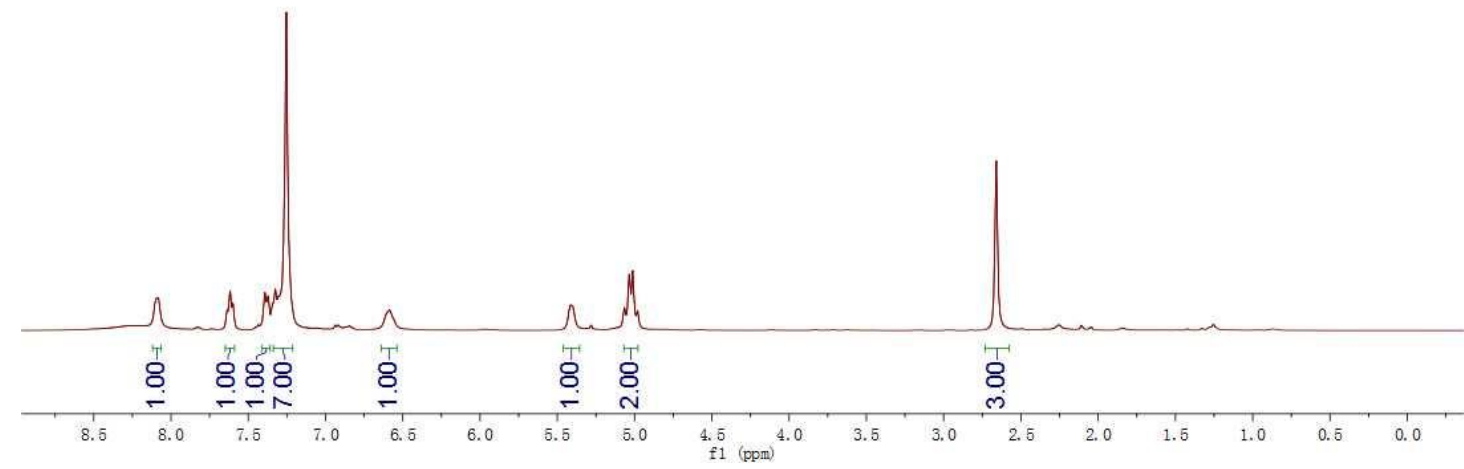

6j- ${ }^{13} \mathrm{C}\{1 \mathrm{H}\}$ NMR (100 $\left.\mathrm{MHz}, \mathrm{CDCl}_{3}\right)$

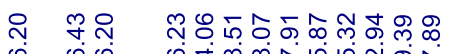

它

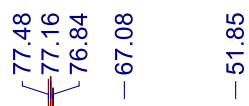

$\underset{\substack{1 \\ \infty}}{\stackrel{1}{\infty}}$<smiles>Cc1oc2ccccc2c(=O)c1[C@H](NC(=O)OCc1ccccc1)C(=O)O</smiles>

6j

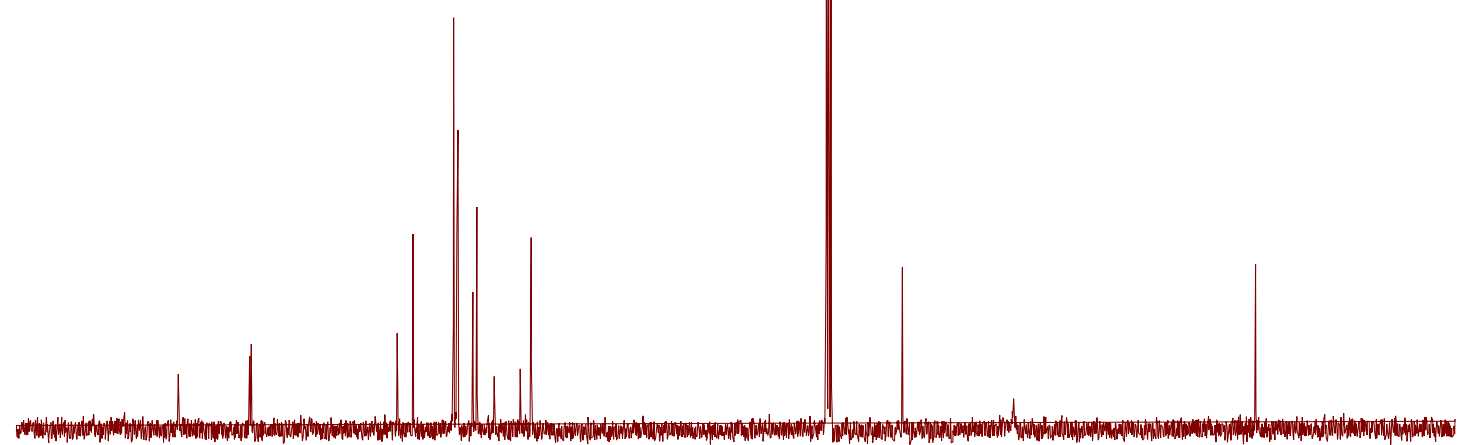

$180 \quad 170 \quad 160$

$50 \quad 140$

$\begin{array}{llll}130 & 120 & 110 & 100\end{array}$

f1 $\stackrel{90}{(\mathrm{ppm})}$ 
6k- ${ }^{1} \mathrm{H}$ NMR (400 $\left.\mathrm{MHz}, \mathrm{CDCl}_{3}\right)$

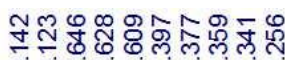

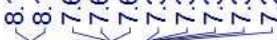

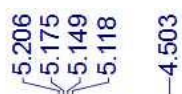

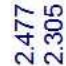

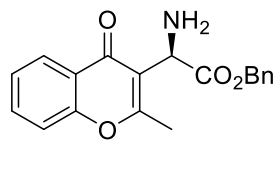

$6 k$

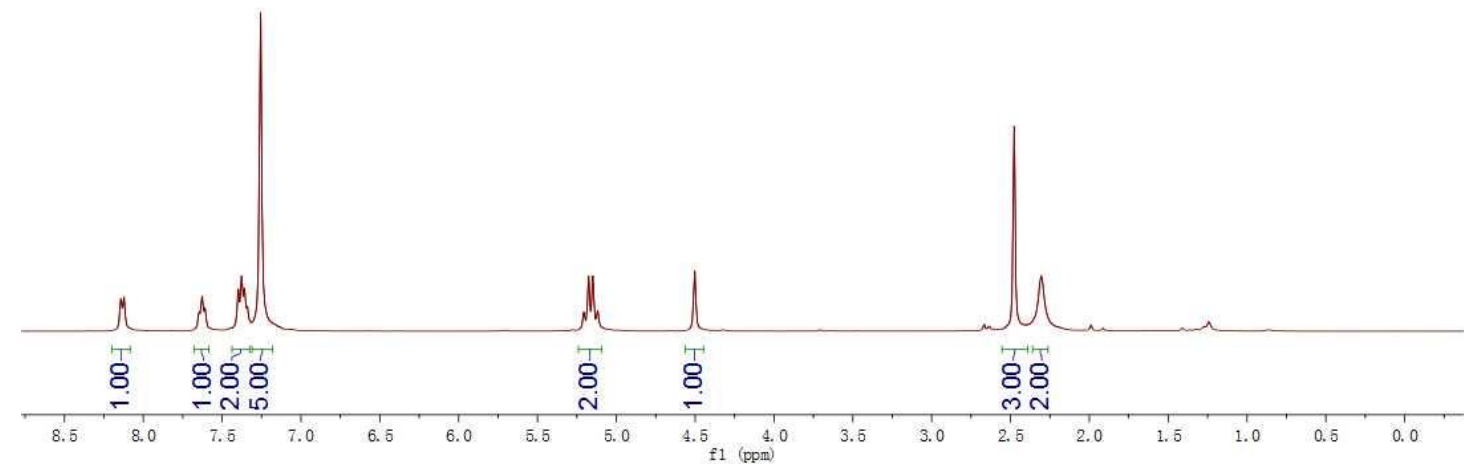

6k- ${ }^{13} \mathrm{C}\{1 \mathrm{H}\}$ NMR (100 $\left.\mathrm{MHz}, \mathrm{CDCl}_{3}\right)$

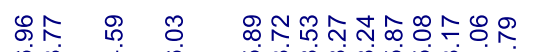

iv

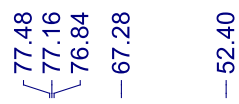

$\frac{10}{10}$

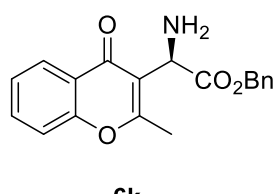

$6 k$
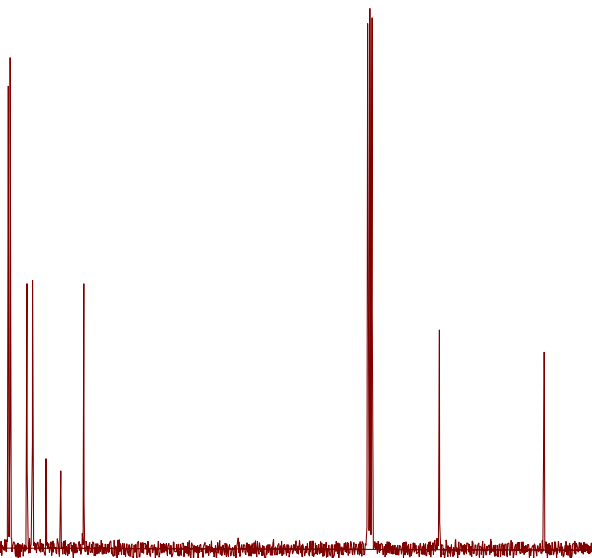
3z- ${ }^{1} \mathrm{H}$ NMR (500 MHz, CDCl3)

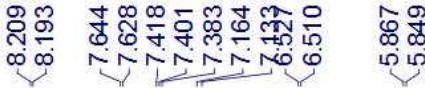

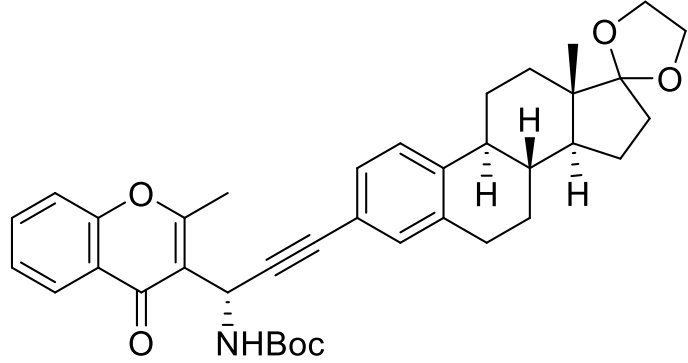

$3 z$

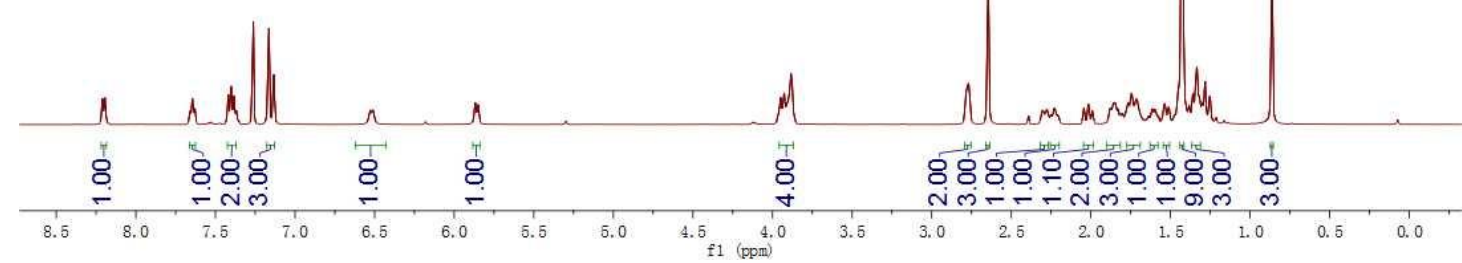

3z- ${ }^{13} \mathrm{C}\{1 \mathrm{H}\}$ NMR (150 MHz, $\left.\mathrm{CDCl}_{3}\right)$

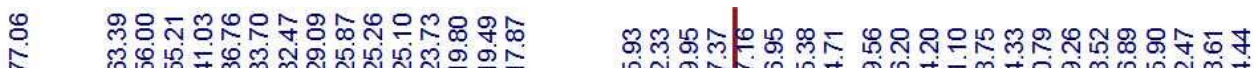

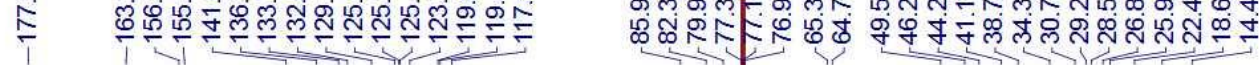

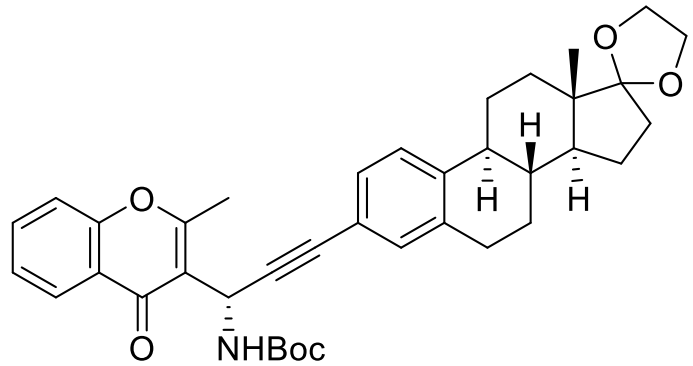

$3 z$

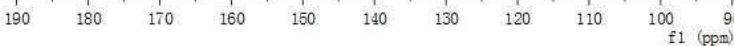


7a- ${ }^{1} \mathrm{H}$ NMR (400 MHz, CDCl3)

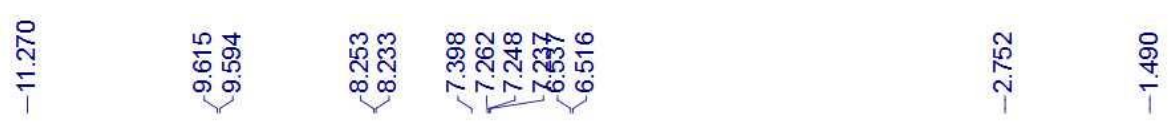<smiles>Cc1oc2ccccc2c(=O)c1[C@@H](C#Cc1ccccc1)NC(=NC(=O)OC(C)(C)C)NC(=O)OC(C)(C)C</smiles>

$7 a$

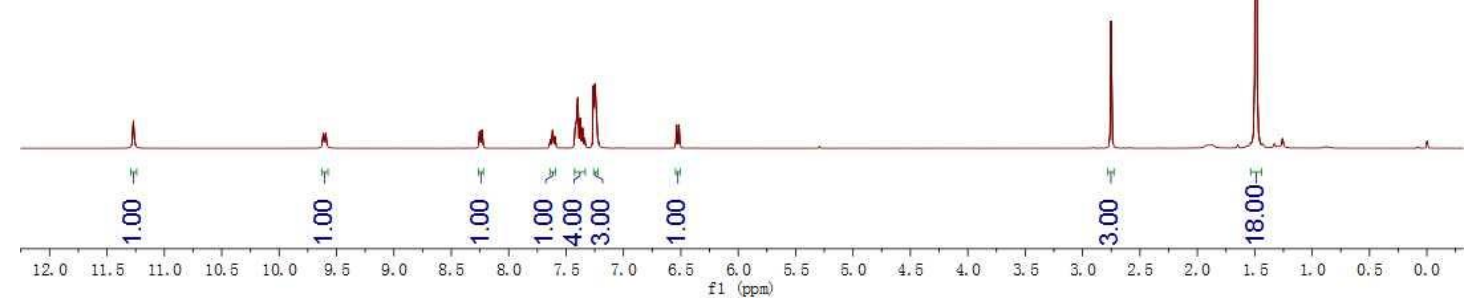

7a- ${ }^{13} \mathrm{C}\{1 \mathrm{H}\}$ NMR (100 MHz, $\left.\mathrm{CDCl}_{3}\right)$

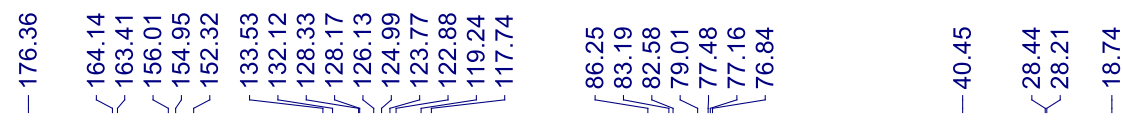<smiles>Cc1oc2ccccc2c(=O)c1[C@@H](C#Cc1ccccc1)NC(=NC(=O)OC(C)(C)C)NC(=O)OC(C)(C)C</smiles>

$7 a$

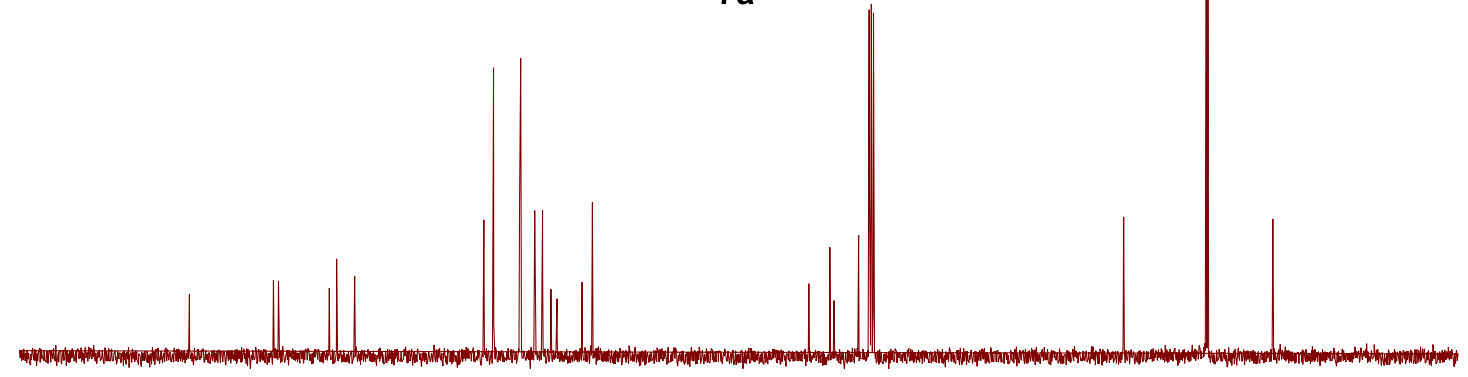

$\begin{array}{lllllllllll}200 & 190 & 180 & 170 & 160 & 150 & 140 & 130 & 120 & 110 & 100 \\ \mathrm{fl}(\mathrm{ppm}) & 90\end{array}$ 
8a- ${ }^{1} \mathrm{H}$ NMR (400 MHz, CDCl3)

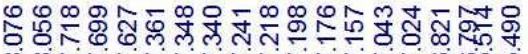<smiles>Cc1oc2ccccc2c(=O)c1[C@H](N)C#Cc1ccccc1</smiles>

$8 a$

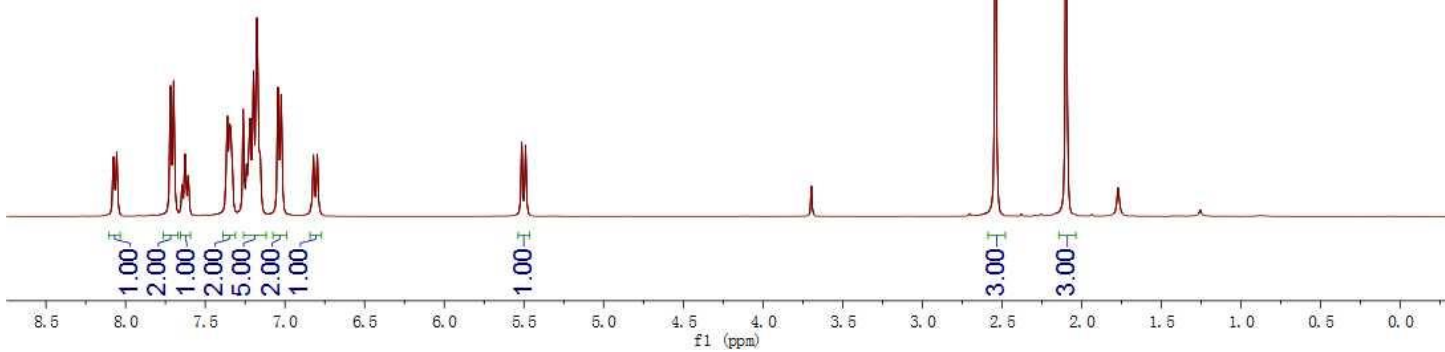

8a- ${ }^{13} \mathrm{C}\{1 \mathrm{H}\}$ NMR (100 MHz, CDCl 3$)$

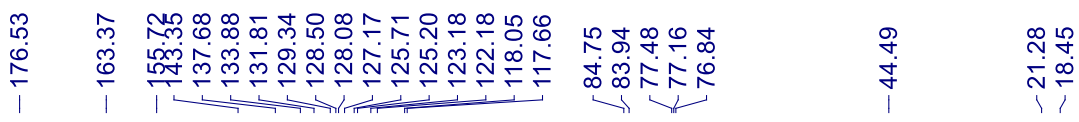

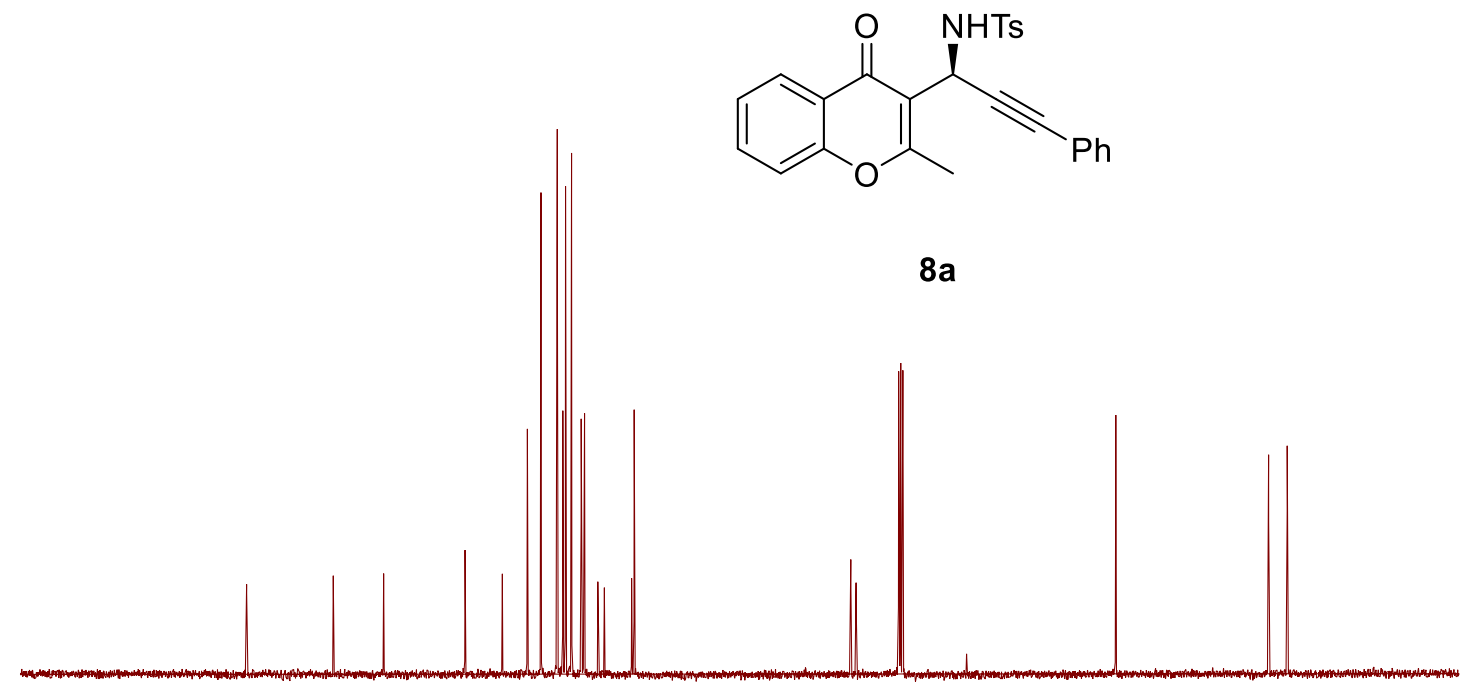

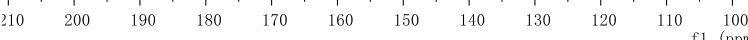


9a- ${ }^{1} \mathrm{H}$ NMR (400 MHz, CDCl3)

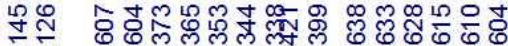

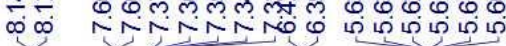

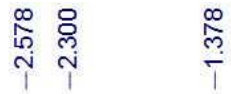<smiles>C#C[C@H](NC(C)(C)C)c1c(C)oc2ccccc2c1=O</smiles>

9a

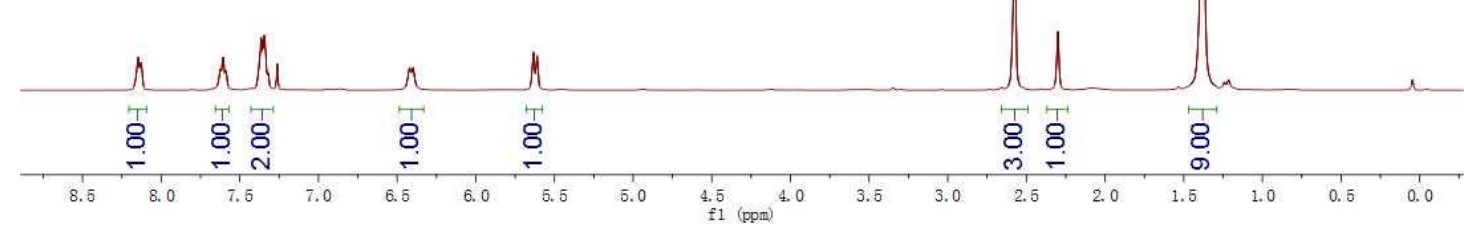

9a- ${ }^{13} \mathrm{C}\{1 \mathrm{H}\}$ NMR (150 MHz, $\left.\mathrm{CDCl}_{3}\right)$

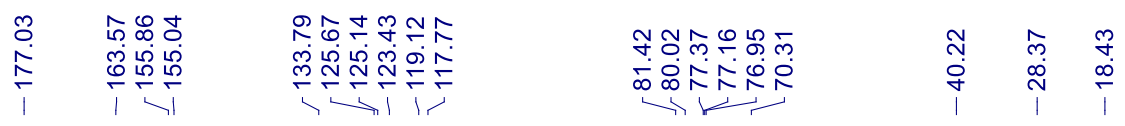<smiles>C#C[C@H](NC(=O)OC(C)(C)C)c1c(C)oc2ccccc2c1=O</smiles>

$9 a$
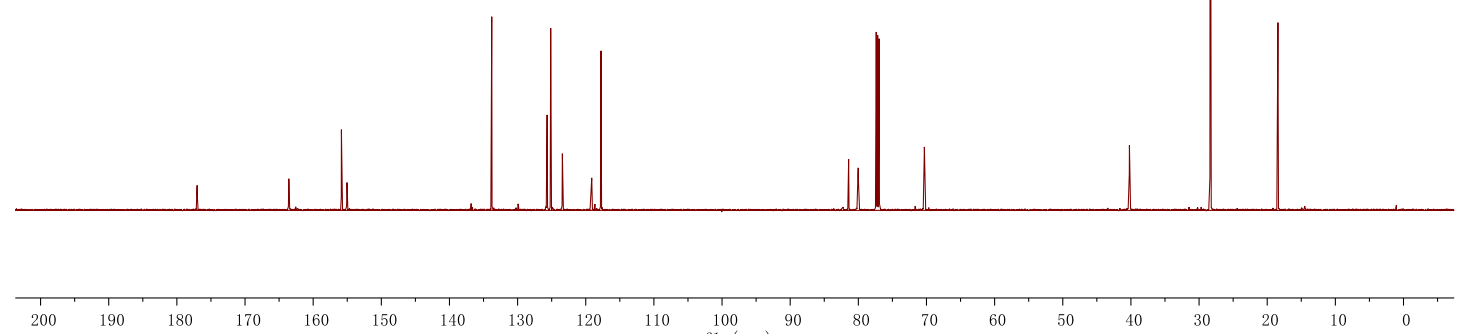

$\mathrm{fl}_{\mathrm{f}(\mathrm{ppm})}^{100} 90$ 
9b- ${ }^{1} \mathrm{H}$ NMR (400 MHz, $\mathrm{CDCl}_{3}$ )

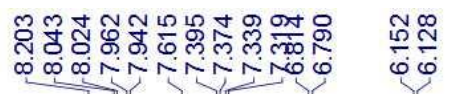

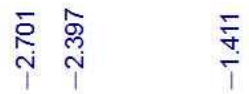<smiles>Cc1oc2ccccc2c(=O)c1[C@H](N)c1cn(S)nn1</smiles>

$9 b$

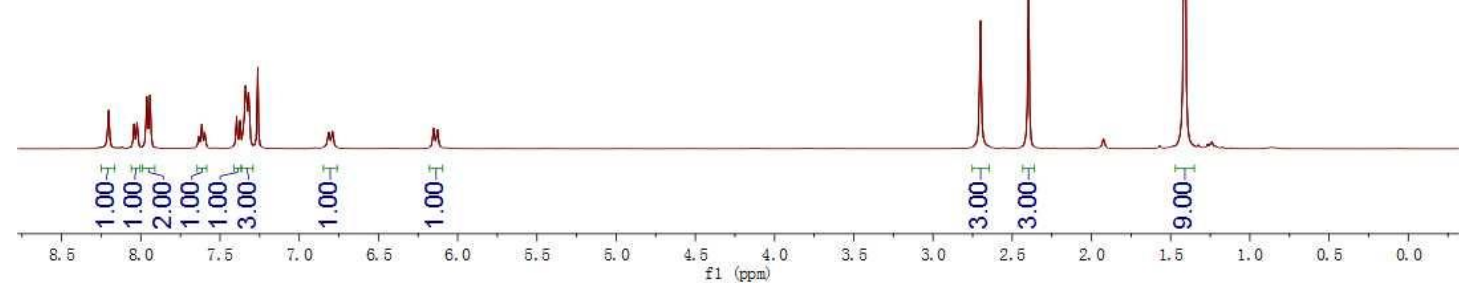

9b- ${ }^{13} \mathrm{C}\{1 \mathrm{H}\}$ NMR (100 $\left.\mathrm{MHz}, \mathrm{CDCl}_{3}\right)$

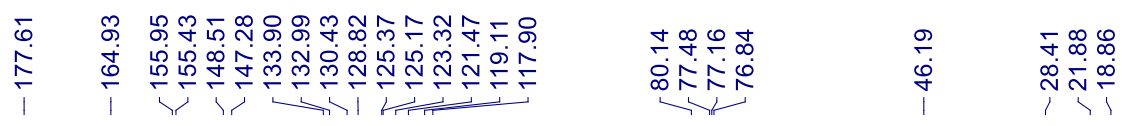<smiles>Cc1oc2ccccc2c(=O)c1[C@H](N)C(=O)OC(C)(C)C</smiles>

$9 b$

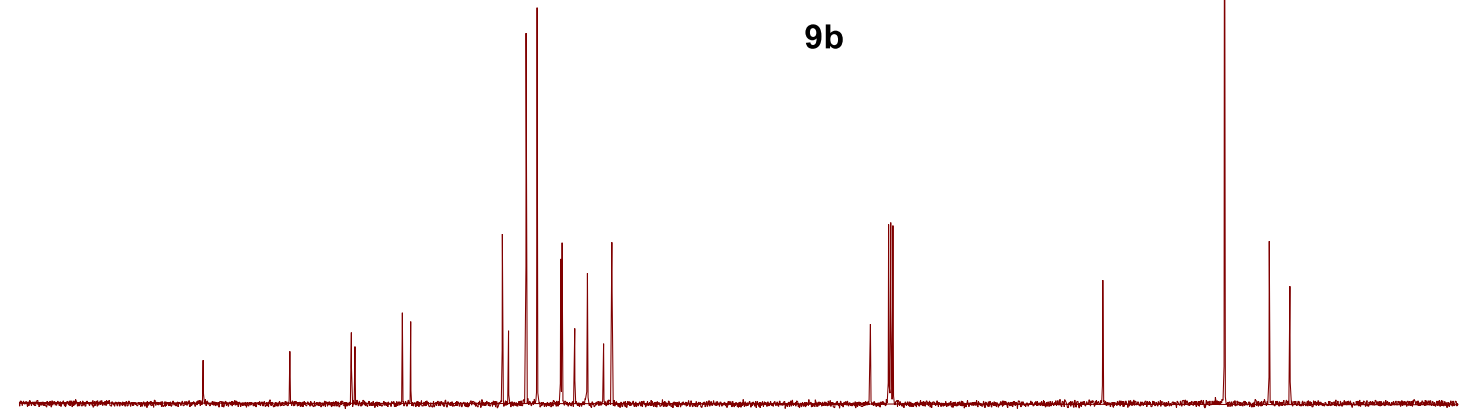

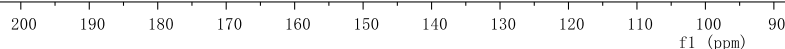


9c- ${ }^{1} \mathrm{H}$ NMR (400 MHz, CDCl3)

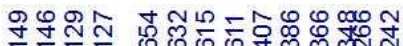

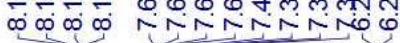

유윯용

itiz

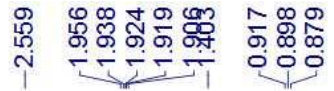

(N)

$9 c$

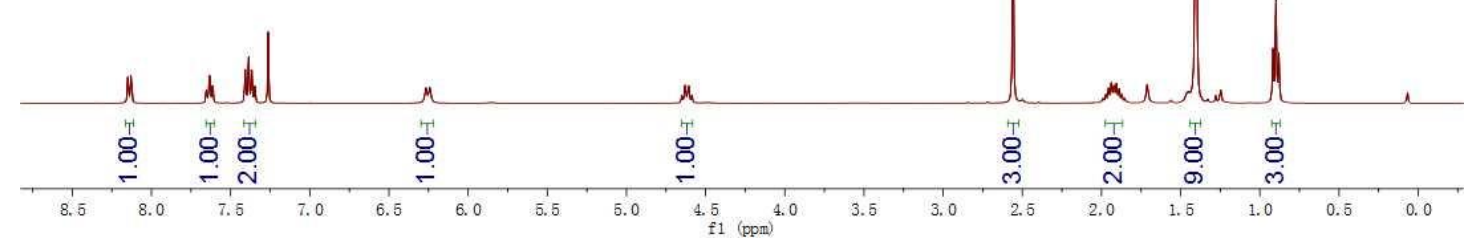

9c- ${ }^{13} \mathrm{C}\{1 \mathrm{H}\}$ NMR (150 $\left.\mathrm{MHz}, \mathrm{CDCl}_{3}\right)$

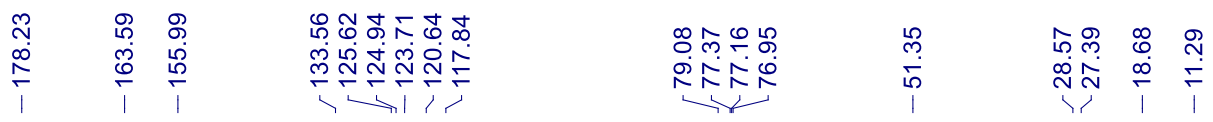<smiles>CC[C@H](N)c1c(C)oc2ccccc2c1=O</smiles>

$9 c$

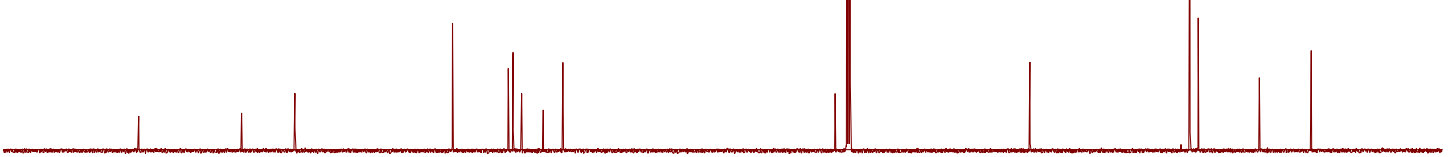

$\begin{array}{lllllllllll}190 & 180 & 170 & 160 & 150 & 140 & 130 & 120 & 110 & 100 & 90 \\ \mathrm{f} 1 & (\mathrm{pmm})\end{array}$ 


\section{2z- ${ }^{1} \mathrm{H}$ NMR (400 MHz, CDCl3)}

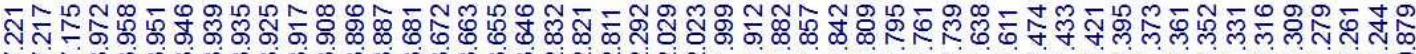

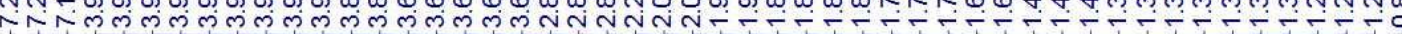

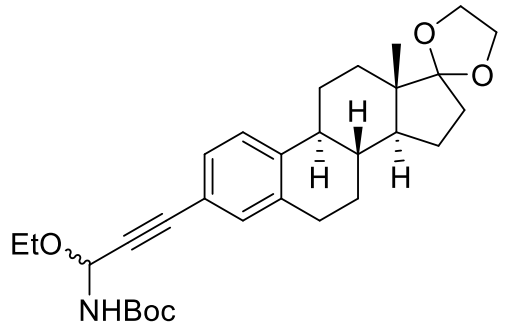

$2 z$

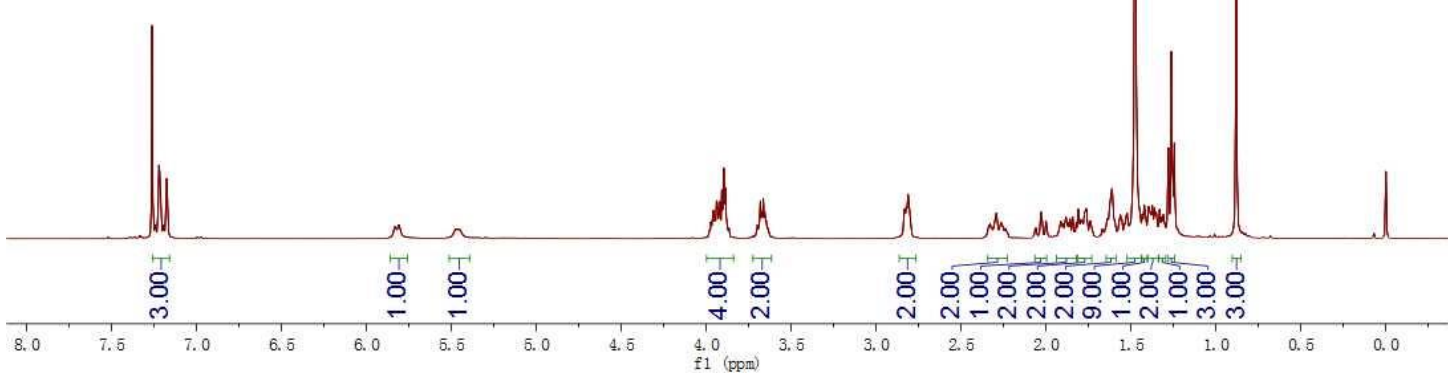

2z- ${ }^{13} \mathrm{C}\{1 \mathrm{H}\}$ NMR (100 MHz, $\left.\mathrm{CDCl}_{3}\right)$

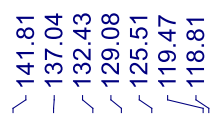

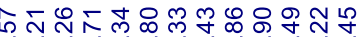

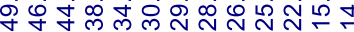

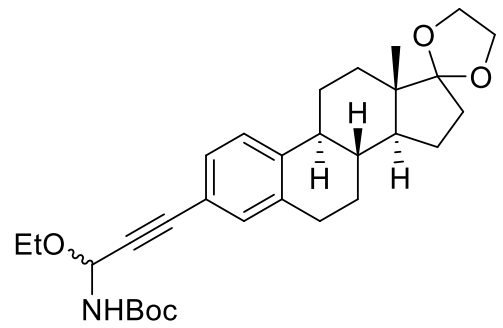

$2 z$
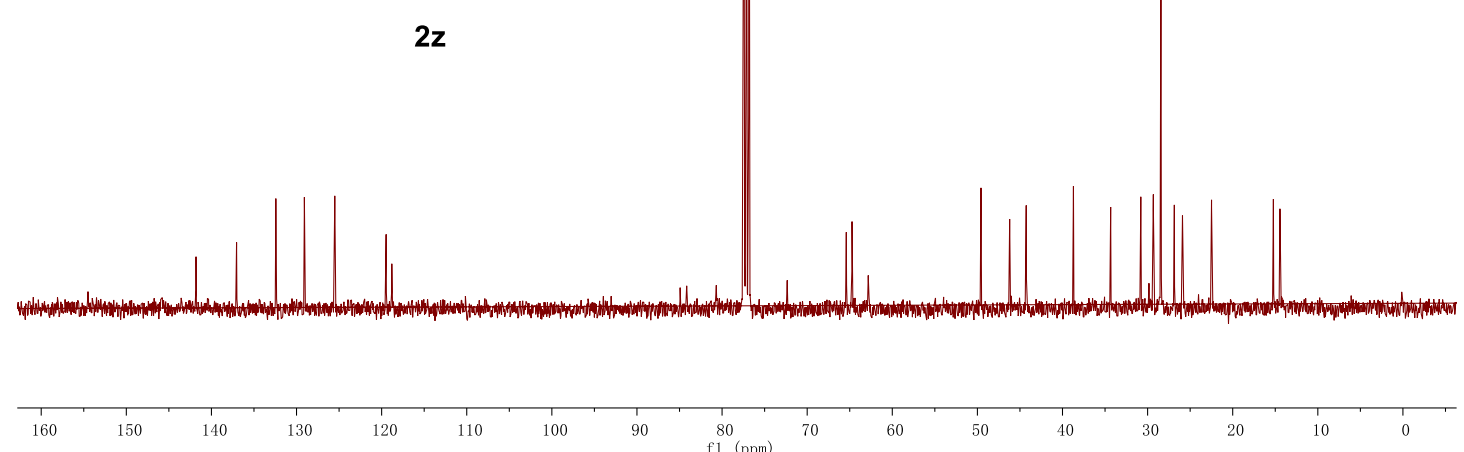
5a- ${ }^{1} \mathrm{H}$ NMR (400 MHz, CDCl 3$)$

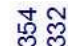

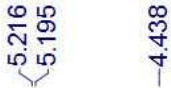

ঙ্ণ

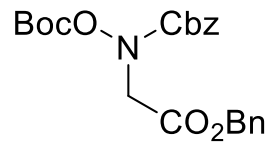

$5 a$

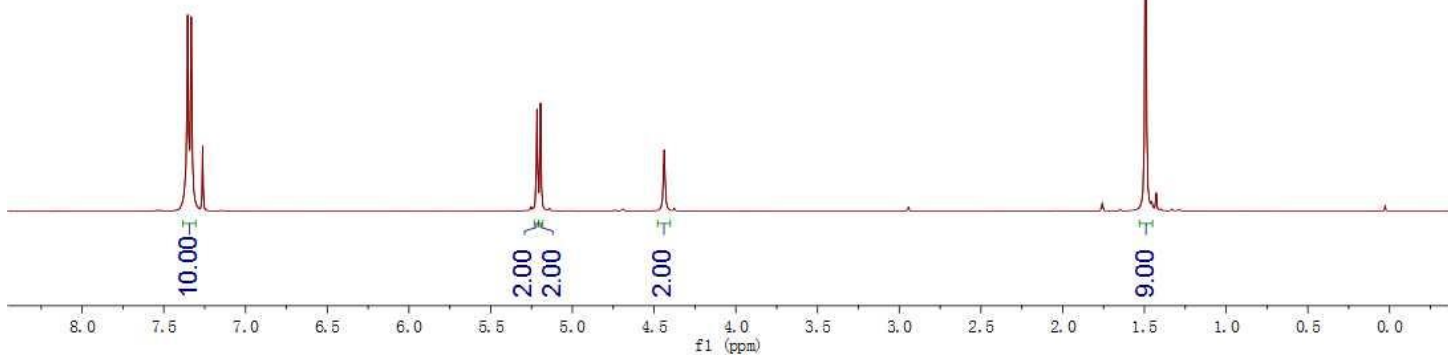

5a- ${ }^{13} \mathrm{C}\{1 \mathrm{H}\}$ NMR (100 $\left.\mathrm{MHz}, \mathrm{CDCl}_{3}\right)$

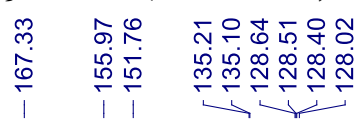

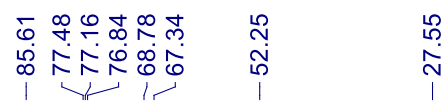
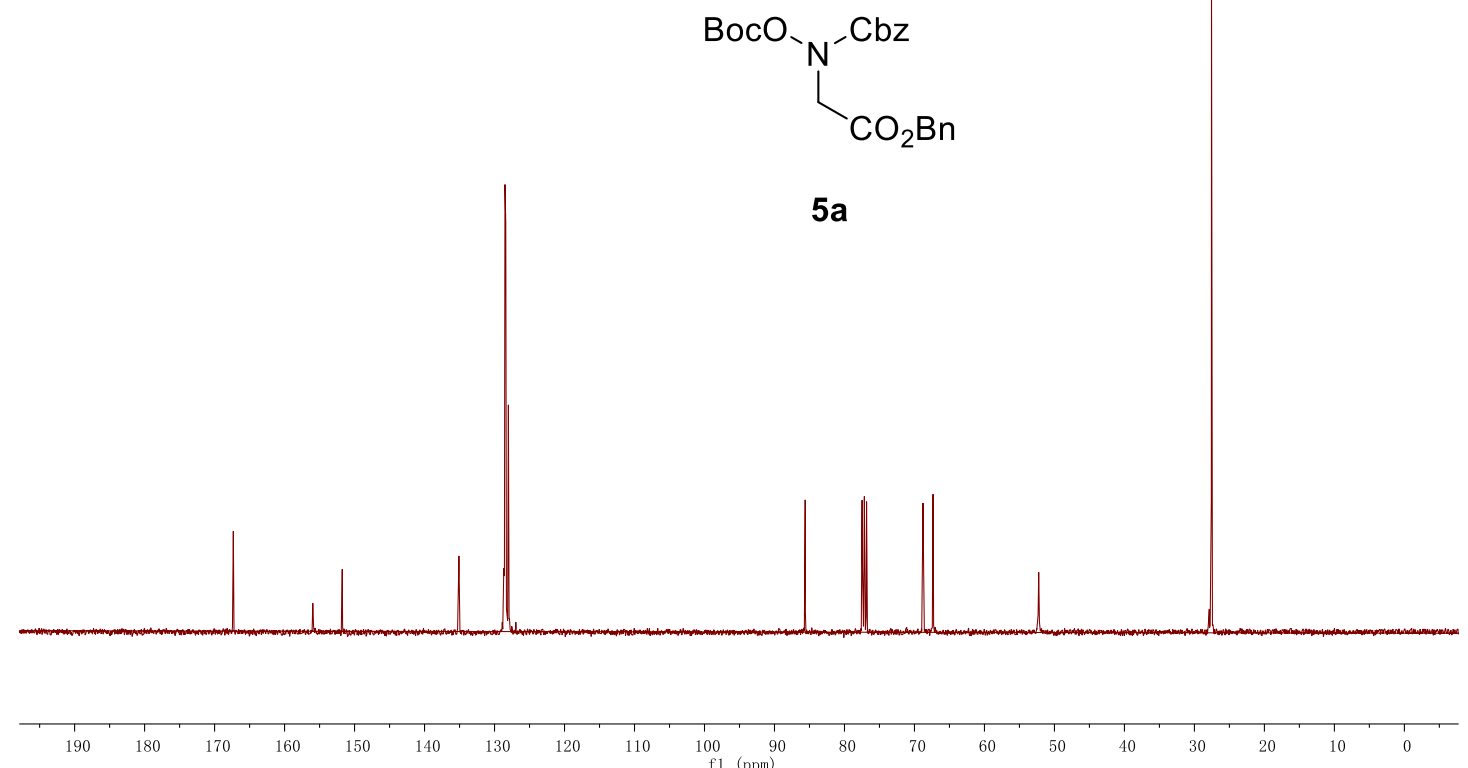